\title{
Nonlinear pulse compression
}

\author{
DISSERTATION \\ presented for the degree of \\ Doctor of Philosophy \\ by
}

Alexander Grün

ICFO - Institut de Ciències Fotòniques

UPC - Universitat Politècnica de Catalunya

Thesis Advisor: Prof. Dr. Jens Biegert 



\section{Abstract}

In this thesis I investigate two methods for generating ultrashort laser pulses in spectral regions which are ordinarily difficult to achieve by the existing techniques. These pulses are especially attractive in the study of ultrafast (few femtosecond) atomic and molecular dynamics.

The first involves Optical Parametric Amplification (OPA) mediated by four-wave-mixing in gas and supports the generation of ultrashort pulses in the Near-InfraRed (NIR) to the Mid-InfraRed (MIR) spectral region. By combining pulses at a centre wavelength of $800 \mathrm{~nm}$ and their second harmonic in an argon-filled hollow-core fibre, we demonstrate near-infrared pulses, peaked at $1.4 \mu \mathrm{m}$, with $5 \mu \mathrm{J}$ energy and 45 fs duration at the fibre output. The four-wave-mixing process involved in the OPA is expected to lead to carrier-envelope phase stable pulses which is of great importance for applications in extreme nonlinear optics. These NIR to MIR pulses can be used directly for nonlinear light-matter interactions making use of its long-wavelength characteristics.

The second method allows the compression of intense femtosecond pulses in the ultraviolet (UV) region by sum-frequency mixing two bandwidth limited NIR pulses in a noncollinear phasematching geometry under particular conditions of group-velocity mismatch. Specifically, the crystal has to be chosen such that the group velocities of the NIR pump pulses, $v_{1}$ and $v_{2}$, and of the sum-frequency generated pulse, $v_{S F}$, meet the following condition: $v_{1}<v_{S F}<v_{2}$. In case of strong energy exchange and an appropriate pre-delay between the pump waves, the leading edge of the faster pump pulse and the trailing edge of the slower one are depleted. As a consequence the temporal overlap region of the pump pulses remains narrow, resulting in the shortening of the upconverted pulse. The noncollinear beam geometry allows the control of the relative group velocities while maintaining the phasematching condition. To ensure parallel wavefronts inside the crystal and the generation of untilted sum-frequency pulses, precompensation of the NIR pulse-front tilts is essential. I show that these pulse-front tilts can be achieved using a very compact setup based on 
transmission gratings and a more complex setup based on prisms combined with telescopes. UV pulses as short as $32 \mathrm{fs}$ ( $25 \mathrm{fs}$ ) have been generated by noncollinear nonlinear pulse compression in a type II phasematching BBO crystal, starting with NIR pulses of $74 \mathrm{fs} \mathrm{(46} \mathrm{fs)} \mathrm{duration.}$ This is of interest, because there is no crystal that can be used for nonlinear pulse compression at wavelengths near $800 \mathrm{~nm}$ in a collinear geometry. Compared to state-of-the-art compression techniques based on self-phase modulation, pulse compression by sum-frequency generation is free of aperture limitation, and thus scalable in energy. Such femtosecond pulses in the visible and in the ultraviolet are very desirable for studying ultrafast dynamics of a variety of (bio)molecular systems. 


\section{Resumen}

En esta tesis he investigado dos métodos para generar pulsos láser ultracortos en regiones espectrales que son típicamente difíciles de lograr con las técnicas existentes. Estos pulsos son especialmente atractivos en el estudio de la dinámica ultrarrápida (pocos femtosegundos) en átomos y moléculas.

La primera técnica implica Amplificación Paramétrica Óptica (OPA) mediante mezcla de cuatro ondas en fase gaseosa y soporta la generación de pulsos ultracortos desde el Infrarrojo-Cercano (NIR) hasta la región espectral del Infrarrojo-Medio (MIR). Mediante la combinación de pulsos centrados a una longitud de onda de $800 \mathrm{~nm}$ y su segundo armónico en una fibra hueca rellena de argón, hemos demostrado a la salida de la fibra la generación de pulsos en el NIR, centrados a $1.4 \mu \mathrm{m}$, con $5 \mu \mathrm{J}$ de energía y 45 fs de duración. Se espera que el proceso de mezcla de cuatro ondas involucrado en el OPA lleve a pulsos con fase de la envolvente de la portadora estables, ya que es de gran importancia para aplicaciones en óptica extrema no lineal. Estos pulsos desde el NIR hasta el MIR se pueden utilizar directamente en interacciones no-lineales materia-radiación, haciendo uso de sus características de longitud de onda largas.

El segundo método permite la compresión de pulsos intensos de femtosegundos en la región del ultravioleta (UV) mediante la mezcla de suma de frecuencias de dos pulsos en el NIR limitados en el ancho de banda en una geometría de ajuste de fases no-colineal bajo condiciones particulares de discrepancia de velocidades de grupo. Específicamente, el cristal debe ser elegido de tal manera que las velocidades de grupo de los pulsos de bombeo del NIR, $v_{1}$ y $v_{2}$, y la del pulso suma-de-frecuencias generado, $v_{S F}$, cumplan la siguiente condición, $v_{1}<v_{S F}<v_{2}$. En el caso de un fuerte intercambio de energía y un pre-retardo adecuado entre las ondas de bombeo, el borde delantero del pulso de bombeo más rápido y el borde trasero del más lento se agotan. De esta manera la región de solapamiento temporal de los impulsos de bombeo permanece estrecha, resultando en el acortamiento del impulso generado. La geometría de haces 
no-colineales permite controlar las velocidades de grupo relativas mientras mantiene la condición de ajuste de fase. Para asegurar frentes de onda paralelos dentro del cristal y que los pulsos generados por suma de frecuencias se generen sin inclinación, es esencial la pre-compensación de la inclinación de los frente de onda de los pulsos NIR. En esta tesis se muestra que estas inclinaciones de los frentes de onda se pueden lograr utilizando una configuración muy compacta basada en rejillas de transmisión y una configuración más compleja basada en prismas combinados con telescopios. Pulsos en el UV tan cortos como $32 \mathrm{fs}$ ( $25 \mathrm{fs}$ ) se han generado mediante compresión de pulsos no-lineal no-colineal en un cristal BBO de ajuste de fase tipo II, comenzando con pulsos en el NIR de 74 fs (46 fs) de duración. El interés de este método radica en la inexistencia de cristales que se puedan utilizar para la compresión de impulsos no-lineal a longitudes de onda entorno a $800 \mathrm{~nm}$ en una geometría colineal. En comparación con las técnicas de última generación de compresión basadas en la automodulación de fase, la compresión de pulsos por suma de frecuencias esta libre de restricciones en la apertura de los pulsos, y por lo tanto es expandible en energía. Tales pulsos de femtosegundos en el visible y en el ultravioleta son fuertemente deseados en el estudio de dinámica ultrarrápida de una gran variedad de sistemas (bio)moleculares. 


\section{Acknowledgements}

First and foremost, I would like to express my very great appreciation to Professor Jens Biegert who accepted me as his first Ph.D. student in the Attoscience and Ultrafast Optics (AUO) group at ICFO. He has been a great supervisor, who, despite his tight time schedule, was always there to encourage and support me. Thank you for your invaluable advice and your persistent effort to improve this top-notch laboratory. It was a pleasure to help to build and to work in it.

Secondly, but not less important, Dane R. Austin deserves all my gratitude for investing such enormous amounts of time and energy in helping and explaining physics to me. Without his sharp mind combined with his never-ending MATLAB knowledge, the ability to solve problems effectively, and his methodological approach in general, it would not have been possible to achieve our ambitious goals. I consider myself very fortunate to have worked with and learned from such an experienced scientist.

As for the other members of the AUO group, huge thanks to Seth L. Cousin, who has been the greatest to work with, for the good times on the beach, on the mountains, and wherever else. A very big thank you goes to Philip K. Bates who's been a great motivator, instructor, and researcher, it's a shame he left science. Merci Olivier Chalus for all the efforts in getting the lab up and running and carrying the "heevee" stuff, l've never seen such beautiful feet. Thanks also to everyone else who tried to make the labs an enjoyable environment to work in: In the first place Thorsten Ergler, Matthias Smolarsky and Daniele Faccio and later Stephan M. Teichmann, Francisco Silva, Alexandre Thai, Matthias Baudisch, Michaël Hemmer, and Benjamin Wolter. Special thanks to Judith Dura Diez who raised the moral standards substantially. Thanks also to the numerous MSc and other students who've helped me out as they've passed through the group. I guess it will be hard to run across such an amazing, lively and entertaining workplace again.

My grateful thanks are also extended to Adam Wyatt for the fruitful collaboration and to the people of the Max-Planck-Institut für Kernphysik in 
Heidelberg who helped Phil and me to build a Reactionmicroscope, especially to Claus Dieter Schröter, the living ultra-high vacuum lexicon, for sharing his wisdom.

A great thank you must also go to the director and management of ICFO and all the people working in administration and engineering, in particular Xavier Menino Pizarro, Juli Céspedes i Capdevila, José Carlos Cifuentes González, Carlos Dengra Tineo, and Jonas Noudehou G. Padonou who were always helpful in solving all kinds of technical issues.

Many thanks also to all the other group members and friends outside who made my life and work so much more joyful, particularly Francois for helping out with the vacuum equipment and for all the interesting discussions, Mussie, you sunshine and coequal futbolin partner, and not to forget Igor who received me in difficult times, looked after me and introduced me to the galician world, graciñas.

Thanks to my parents Christiane and Herbert for their support and encouragement throughout my studies and to my brother Max and his family for not being scientists.

Finally, a very special acknowledgement goes to Judith from whom I have received unlimited support. Her help and patience have been greatly appreciated, particularly in the past few months (eres la mejor, de verdad). I hope I can compensate you in some way and I start by dedicating this book to our grandparents, without them none of us would be where we are today. 
"If I could remember the names of all these particles l'd be a botanist."

Enrico Fermi 


\section{Contents}

Abstract i

Resumen

Acknowledgements v v

1 Introduction 1

2 Ultrashort pulses $\quad \mathbf{5}$

2.1 Optical pulse compression . . . . . . . . . . . . . . . 5

2.1.1 Chirped and bandwidth-limited pulses . . . . . . . 5

2.1.2 Self-phase modulation and pulse compression . . . 6

2.1.3 Nonlinear pulse compression . . . . . . . . . . . 11

2.2 Ultrashort UV pulses . . . . . . . . . . . . . . . . . . . . . . 14

2.2.1 Ultrafast UV sources . . . . . . . . . . . . . . . 15

2.2.2 UV applications . . . . . . . . . . . . . . 17

2.3 Ultrashort NIR to MIR pulses . . . . . . . . . . . . . . . . . 19

2.3.1 Ultrafast NIR to MIR sources . . . . . . . . . . . 19

2.3.2 NIR to MIR applications . . . . . . . . . . . . . 21

3 Theory of nonlinear three-wave interaction 25

3.1 The coupled wave equations . . . . . . . . . . . . . . 25

3.2 Phasematching, group velocities and pulse-front tilts . . . . 31

3.2.1 Phasematching . . . . . . . . . . . . . . . 31

3.2.2 Angular dispersion . . . . . . . . . . . . . 32

3.2 .3 Group velocities . . . . . . . . . . . . . . . 34

3.2.4 Pulse-front tilt . . . . . . . . . . . . . . 35

4 Numerical simulations 41

4.1 Simulation properties and parameters . . . . . . . . . . . 41

4.2 Numerical results of the simulation . . . . . . . . . . . . . . 44

4.2.1 Strong energy exchange . . . . . . . . . . . 45

4.2.2 Dependence of the crystal cut angle . . . . . . . . 46

4.2.3 Pre-delay ................ . . 48 
4.2.4 Dispersion and nonlinear phase . . . . . . . . 50

4.2 .5 Pulse Evolution in the crystal . . . . . . . . . . . 52

4.2.6 Shortening of the fundamental frequency pulses . . 54

4.2.7 Reconstructed pulses . . . . . . . . . . . . . 56

5 Femtosecond laser system and diagnostics 61

5.1 Ti:sapphire amplifier laser system . . . . . . . . . . . 61

5.1 .1 Chirped pulse amplification . . . . . . . . . . 62

5.1 .2 Oscillator . . . . . . . . . . . . . . 63

5.1 .3 Stretcher . . . . . . . . . . . . . . . . 64

5.1 .4 Amplifier . . . . . . . . . . . . . . . . . 65

5.1 .5 Grating compressor . . . . . . . . . . . . . . 67

5.2 Femtosecond pulse diagnostics . . . . . . . . . . . . 68

5.2.1 Second-harmonic generation FROG . . . . . . . 69

5.2 .2 Self-diffraction $F R O G \ldots \ldots \ldots . \ldots 71$

5.2 .3 SEA-SPIDER . . . . . . . . . . . . . . . . 74

5.2 .4 Phazzler. . . . . . . . . . . . . . . . . 78

6 Near-infrared OPA in a gaseous medium 83

6.1 Experimental setup . . . . . . . . . . . . . . . . . 83

6.2 Results . . . . . . . . . . . . . . . . . . . . . 84

6.3 Discussion and conclusion . . . . . . . . . . . 87

7 Nonlinear pulse compression $\quad 91$

7.1 Experimental setups . . . . . . . . . . . . . . . . . . . 91

7.2 Results ....................... . . . 98

7.2.1 Compression of 74 fs long NIR pulses . . . . . . 98

7.2.2 Compression of sub $50 \mathrm{fs}$ NIR pulses ........ 104

7.2 .3 Discussion . . . . . . . . . . . . . . . . 108

7.3 Conclusion . . . . . . . . . . . . . . . . . 111

8 Summary and outlook 113

$\begin{array}{ll}\text { A Transmission grating damage report } & 117\end{array}$

$\begin{array}{ll}\text { B Matlab code } & 121\end{array}$

$\begin{array}{ll}\text { Acronyms } & 125\end{array}$

$\begin{array}{ll}\text { References } & 126\end{array}$

$\begin{array}{ll}\text { Publications } & 143\end{array}$ 


\section{Chapter 1}

\section{Introduction}

Since several years there is great interest in the physics of ultrashort ${ }^{1}$ laser pulses. The prefix ultra originates from the Latin ulter, meaning beyond, and anticipates the outstanding role of ultrafast lasers as enabling tool for countless scientific and commercial applications. Ultrashort pulses tend to possess extremely high peak intensities and thus can excite all sorts of nonlinear processes. The latter are for example frequency mixing processes like sum-frequency generation (SFG) and optical parametric amplification (OPA) or other nonlinear processes like the optical Kerr effect or cross-phase modulation (XPM), just to name some of them. Amplified pulses of shortest duration down to the few-cycle regime allow the generation of intense coherent sources in the extreme ultraviolet (XUV) to X-ray regime and the formation of single attosecond $\left(1 \mathrm{as}=10^{-18} \mathrm{~s}\right)$ pulses, with pulse durations below 100 as. ${ }^{1-3}$ Besides exciting nonlinear processes ultrashort laser pulses can be used to probe the properties of matter on extremely short time scales. Pulses in the femtosecond range led to the development of femtochemistry, making it possible to measure the dynamics of molecules and solids in real time, for which the 1999 Nobel Prize in Chemistry was awarded to Prof. Ahmed Zewail. Pulses in the attosecond range led to the development of attoscience, making it possible to track processes inside atoms and to study the motion of electrons.

The progress in ultrafast compact lasers during the past decade has been simply amazing. Historically ultrashort laser pulses were first generated by dye lasers and dominated the market until the discovery of the Ti:sapphire laser in the late 1980s. Nowadays such pulses are also gener-

\footnotetext{
${ }^{1}$ In this thesis the label ultrashort applies to electromagnetic pulses whose time duration is in the domain of femtoseconds $\left(1 \mathrm{fs}=10^{-15} \mathrm{~s}\right)$.
} 
ated with optical parametric amplifiers, possibly using a supercontinuum ${ }^{2}$ as input, or with free electron lasers. Ultrafast lasers can be found for a wide range of wavelengths with pulse durations down to a single cycle. Although the diversity of ultrafast laser systems is increasing, Ti:sapphire remains the gain media of choice for the overwhelming majority of scientific applications, due to its unsurpassed combination of performance capability, versatility and value. Nevertheless there is a drawback, due to the finite spectral bandwidth of Ti:sapphire laser amplifiers, the pulse duration is limited to about $15 \mathrm{fs} .{ }^{4}$ Shorter pulses would be of interest because they could lead to higher peak powers without increasing the laser-pulse energy, e.g., without increasing the size and cost of the laser system.

To date, a variety of methods exist for further (external) optical pulse compression (shortening). Typically they are based on the interplay between spectral broadening by Kerr induced self-phase modulation (SPM) and a negative dispersive delay line for temporal compression. Traditionally, hollow core fibres ${ }^{5}$ and filamentation ${ }^{6}$ in combination with chirped mirrors are widely used methods for compressing pulses below $5 \mathrm{fs}$. This methods are limited to sub-milijoule level due to material damage or intensity clamping. In order to increase the energy throughput, pressure gradients, ${ }^{7}$ planar hollow waveguides ${ }^{8,9}$ and tapered waveguides ${ }^{10}$ were proposed. Yet, the waveguide still imposes a limit on the aperture and thus the power of the compressed pulse.

In this thesis I want to present two alternative methods to generate ultrashort laser pulses: The first method involves optical parametric amplification (OPA) mediated by four-wave-mixing in gas and supports lasing and generation of ultrashort pulses in the near-infrared (NIR) to the midinfrared (MIR) spectral region. The second method is scalable in energy and the pulse compression occurs during the nonlinear process, therefore it is called nonlinear pulse compression (NPC). Proposed at the start of the 1990 s, ${ }^{11,12}$ this process involves the nonlinear interaction of three-waves in appropriate conditions of group-velocity mismatch. Combined with a strong energy exchange between the three waves and a reduced pulse temporal overlap area this can lead to efficient pulse compression.

The remainder of this thesis is organised as follows. Chapter 2 presents the background to the work described in the later chapters, specifically a discussion of pulse compression techniques, laser sources in the UV to mid-IR region and their applications. Chapter 3 develops the theory of nonlinear three-wave interaction in a nonlinear medium and derives the

\footnotetext{
${ }^{2}$ When a short intense pulse propagates through a nonlinear medium, it often undergoes significant spectral broadening.
} 
particular pulse requirements in a noncollinear phasematching geometry. Chapter 4 describes the numerical simulation based on the sum-frequency generation of two ultrashort near-infrared pulses inside a type II $\beta$-Barium Borate crystal. Chapter 5 describes an ultrafast Ti:sapphire amplifier laser system and some of the most prominent methods for the full-field characterization of ultrashort light pulses. Chapter 6 deals with the generation of ultrashort laser pulses in the NIR to MIR wavelength region through optical parametric amplification in a gaseous medium. Chapter 7 details the experimental setups and covers the main experimental results leading to nonlinear pulse compression. The final chapter, chapter 8 , presents a summary, conclusions, and some of the possible future work that could be undertaken. 
This chapter is organised as follows: section 2.1 introduces the most important optical pulse compression techniques. Existing laser sources delivering ultrashort pulses in the ultraviolet and their applications are described in section 2.2 and section 2.3 deals with ultrafast near-infrared to mid-infrared laser sources and their applications. 


\section{Chapter 2}

\section{Ultrashort pulses}

\subsection{Optical pulse compression}

\subsubsection{Chirped and bandwidth-limited pulses}

The minimum possible pulse duration of a pulse is set by its spectral bandwidth. This happens when all frequency components have a constant phase across the energy spectrum of the pulse. In other words, when the peak power is as high as the spectral bandwidth allows for a given pulse energy. Such pulses are called bandwidth-limited pulses or Fouriertransform-limited pulse, or, more commonly, transform-limited pulse. To estimate the Fourier limit of a pulse the time-bandwidth can be taken as an approximation. For Fourier-transform-limited Gaussian pulses the timebandwidth product applies as follows; $\tau_{F W H M} \Delta \nu_{F W H M} \approx 0.44$. For example, a bandwidth-limited Gaussian pulse with a duration of 20 fs must have a bandwidth of $22 \mathrm{THz}$, which corresponds to $47 \mathrm{~nm}$ when the centre of the spectrum lies at $800 \mathrm{~nm}$. A transform-limited pulse cannot be further compressed.

Let us assume an optical medium with normal dispersion, i.e. the index of refraction $n$ of the substance decreases with increasing wavelength $(d n / d \lambda<0)^{1}$. If the relative position of the spectral components, the spectral phase, is not constant, then the pulse is called chirped. When a pulse is negatively chirped, the front part of the pulse $(t<0)$ oscillates with a higher frequency than the back part of the pulse $(t>0)$ and vice versa if the pulse is positively chirped (see Fig. 2.1). The pulse duration of a chirped pulse can be easily shortened by sending the pulse through a dis-

\footnotetext{
${ }^{1}$ Whereas, if the index of refraction increases with increasing wavelength, the medium is said to have anomalous dispersion.
} 
(a)

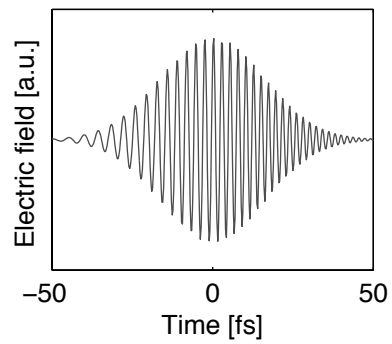

(b)

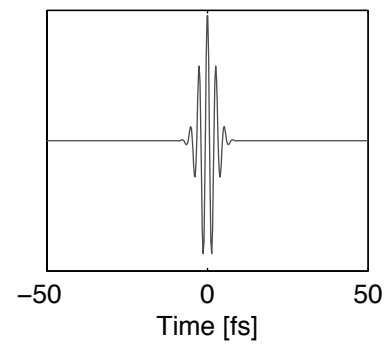

(c)

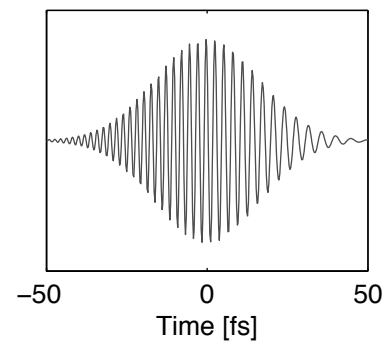

Figure 2.1: Influence of the chirp on a pulse. (a) negatively chirped, (b) chirp free and (c) positively chirped.

persive delay line, for instance a pair of diffraction gratings, ${ }^{13,14}$ a prism pair ${ }^{15}$ or a chirped mirror. The field dealing with removing or at least reducing the chirp is denoted by linear compression.

\subsubsection{Self-phase modulation and pulse compression}

Earlier I said that bandwidth-limited pulses cannot be further compressed, which is only half of the story. More than four decades ago, Gires and Tournois ${ }^{16}$ and Giordmaine et al. ${ }^{17}$ proposed independently that optical pulses can be shortened by adapting microwave pulse compression techniques to the visible spectrum. Optical pulse compression of a transformlimited pulse can be achieved by increasing the optical bandwidth of the initial pulse using a nonlinear interaction such as self-phase modulation. Self-phase modulation is the change in the phase of an optical pulse resulting from the varying refractive index, $n(t)$, of the medium ${ }^{18}$ of the sort

$$
n(t)=n_{0}+n_{2} I(t),
$$

where $n_{0}$ is the linear-, $n_{2}$ the nonlinear refractive index, and $I(t)$ is the time dependent optical intensity. Assuming that the response of the medium to the pulse intensity is essentially instantaneous and that no optical reshaping of the pulse occurs, i.e. that the only effect of the nonlinear medium is to change the phase of the transmitted pulse, then the acquired nonlinear phase $\Phi_{N L}$ can be written as

$$
\Phi_{N L}(t)=-\frac{\omega}{c} n_{2} I(t) L,
$$

where $\omega$ is the frequency, $L$ is the distance travelled in the Kerr medium, and $c$ is the speed of light. Since the pulse intensity rises very steeply a 
frequency chirp is impressed on the pulse carrier which can be described by the variation of the instantaneous frequency ${ }^{2}$

$$
\delta \omega(t)=\frac{d}{d t} \Phi_{N L}(t) .
$$

As a result of this time-varying phase, the spectrum of the pulse typically gets broader and positively chirped, with the transmitted pulse being longer than the original pulse. One problem with this scheme is that other nonlinear processes such as self-focusing can interfere, making a guiding nonlinear medium a fundamental requirement for pulse compression. The absence of a guide would lead to a spatially non-uniform self-phase modulation, so that different frequency chirps are generated across the transverse beam distribution. The final pulse compression, i.e. flattening of the spectral phase, is then achieved by compensating the positive chirp by means of a dispersive line with negative group-velocity dispersion (GVD).

In the last three decades this general scheme of pulse compression, illustrated in Fig. 2.2, has been implemented in different ways. The two most successful techniques, namely, the hollow-fibre compression technique and filamentation-based pulse compression, are presented here.

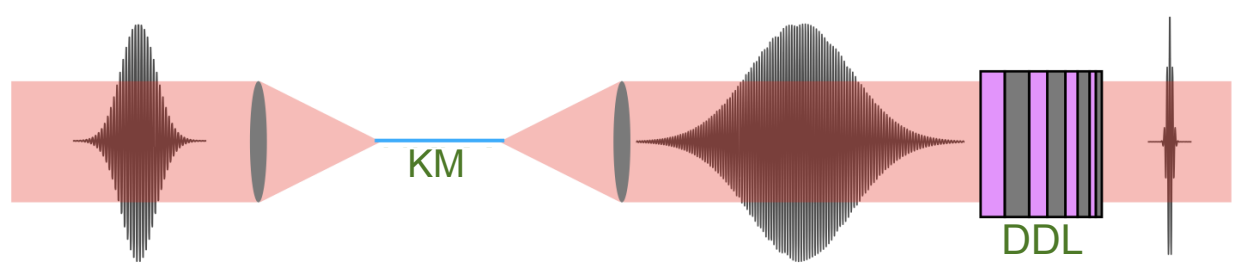

Figure 2.2: Schematic representation of optical pulse compression. The figure depicts the evolution of the electric field. The bandwidth-limited input pulse moves from left to right. The passage through a Kerr medium (KM) spectrally broadens the pulse due to self-phase modulation. Passing the pulse through a dispersive delay line (DDL) with negative groupdelay dispersion (GDD) results in temporal compression.

\section{Hollow core fibre compression}

Nonlinear optical interactions in hollow core fibres (HCFs) and subsequent compression were first performed by Nakatsuka et al. ${ }^{19,20}$ in 1981. They

\footnotetext{
${ }^{2}$ The concept of the instantaneous frequency $\omega(t)$ itself can be described by $\omega(t)=$ $\omega_{0}+\delta \omega(t)$.
} 
used a $70 \mathrm{~m}$ long single-mode optical fibre as Kerr medium in the positive dispersion region for broadening followed by a near-resonant atomic sodium-vapour delay line for compressing 3 ps long pulses down to $1.5 \mathrm{ps}$. In 1982 Shank et al. ${ }^{21}$ showed the first compression of femtosecond optical pulses through single-mode fibres combined with a grating pair. Improving the higher-order dispersion control by means of prisms in combination with gratings culminated in 6 fs short pulses in 1987. This long standing record of the shortest optical pulse by Fork et al. ${ }^{22}$ has been broken by Baltuška et al. ${ }^{23}$ in 1997.13 fs pulses from a cavity-dumped Ti:sapphire laser were compressed down to 4.5 fs with the same technique using a compressor consisting of a quartz $45^{\circ}$ prism pair, broadband chirped mirrors and a thin-film Gires-Tournois dielectric interferometer compressor. The use of single-mode fibres is however limited to low energy nanojoule pulses due to both material damage and appearance of higher-order nonlinearities. In 1996 the group of De Silvestri in Milan developed a powerful pulse compression technique ${ }^{24}$ based on spectral broadening in a hollow fibre filled with noble gases and with the capability of handling high-energy pulses (submillijoule range). The modes of the hollow core fibre are leaky modes, i.e. they experience radiation loss. However, there is one mode, the EH11 mode, which has considerably less loss than the higher order modes. This mode is used for pulse compression. The nonlinear index in the fibre can be controlled by changing the gas type and pressure. This is important for optimizing the nonlinear interaction and both medium and waveguide dispersion. ${ }^{25}$ Typical fibre diameters are 100-500 $\mu \mathrm{m}$ and typical gas pressures are in the range of 0.1-3 bar. Chirped mirrors are capable of controlling the frequency dependent group delay over such a broad bandwidth for subsequent dispersive compression. The implementation of this technique has led to the generation of intense few-cycle laser pulses with a controlled

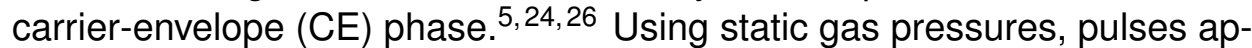
proaching the single-cycle limit have been reported. ${ }^{27,28}$ Yet the occurrence of self-focusing and ionization of the gas medium near the entrance to the fibre limit the energy to a few microjoules. Suda et al. proposed and demonstrated the use of a hollow core fibre with a pressure gradient ${ }^{29}$ to increase the energy. With this technique, the generation of sub-10 fs pulses with energies in excess of $1 \mathrm{~mJ}$ has become feasible, ${ }^{7,29-34}$ e.g., in 2010 Bohman et al. ${ }^{7}$ have generated $5 \mathrm{fs}, 5 \mathrm{~mJ}$ pulses, using helium as nonlinear medium. Figure 2.3 shows a typical pulse generated with a gas filled hollow core fibre and subsequent compression with chirped mirrors. Here, we focused 30 fs long pulses from a Ti:sapphire amplifier system (described in $\S 5.1$ in Chap. 5) into a $1 \mathrm{~m}$ long hollow core fibre filled with 0.6 bar 
of Argon. The pulses were measured ${ }^{35}$ with a home built SEA-SPIDER ${ }^{3}$.

(a)

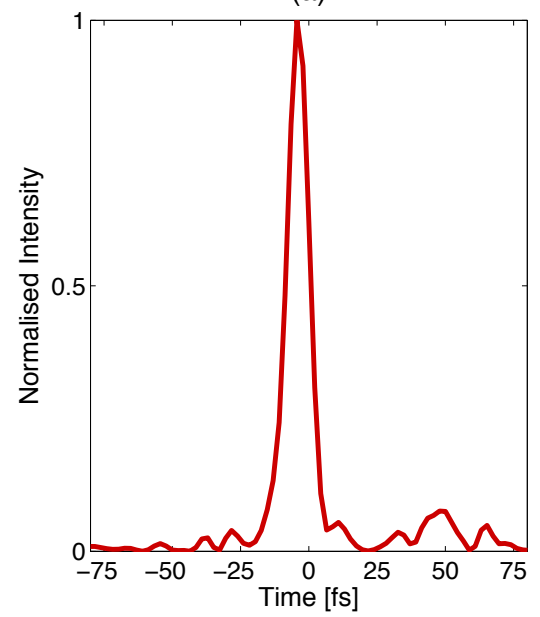

(b)

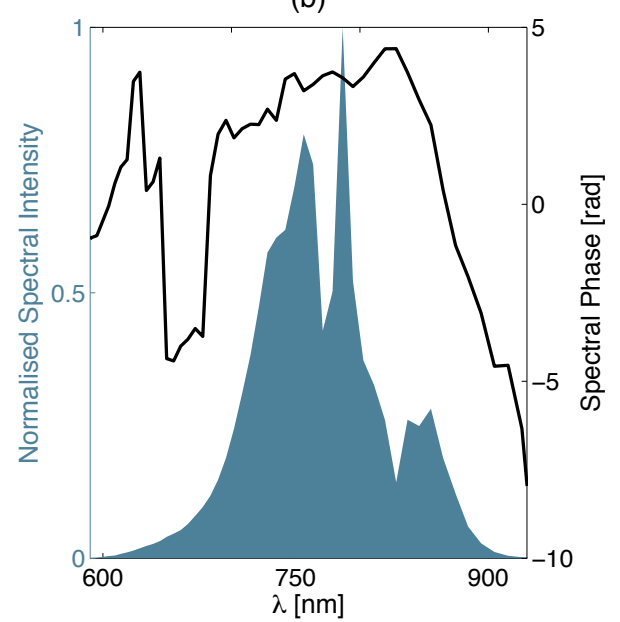

Figure 2.3: Temporal and spectral reconstructions after hollow core fibre compression. $30 \mathrm{fs}$ pulses from a Ti:sapphire amplifier system were compressed down to 9.4 fs in a HCF filled with 0.6 bar of argon.

\section{Filamentation}

Optical filamentation, or self-guiding of femtosecond laser pulses was first discovered by Braun. ${ }^{36}$ When a sufficiently intense laser pulse is loosely focused into a gas-filled cell, a filament forms through a dynamic balance between Kerr-lensing, caused by the intensity dependence of the refractive index of the medium, and defocusing due to diffraction caused by the created plasma. ${ }^{37}$ Simultaneously, the Kerr nonlinearity also acts to broaden the pulse spectrum during propagation in the filament through self-phase modulation. The continuum output can be compressed after the filament using chirped mirrors or other dispersive delay lines. This pulse compression technique is an attractive alternative compared to the core fibre compression scheme for several reasons. Its experimental arrangement is simpler because of the absence of the waveguide, thus removing the difficulty of coupling into a hollow fibre. Associated with the absence of a waveguide and its coupling losses, significantly higher throughput can be obtained compared to hollow core fibres filled with a static pressure. ${ }^{6}$ In addition,

\footnotetext{
${ }^{3}$ Spatially Encoded Arrangement for SPIDER (SEA-SPIDER) is an advancement of Spectral phase interferometry for direct electric-field reconstruction (SPIDER).
} 
self-compression can be observed in certain operation regimes with the exciting prospect of reaching the single-cycle pulse duration regime. ${ }^{38-40}$ Using this method few-cycle pulses of $5.7 \mathrm{fs}$ duration and $0.38 \mathrm{~mJ}$ of energy with a stable CE phase have been demonstrated. ${ }^{6}$ Cascading filaments has led to the generation of $5 \mathrm{fs}, 0.7 \mathrm{~mJ}$ pulses at $1 \mathrm{kHz} .{ }^{41}$ Selfcompression of 45 fs NIR pulses down to 7.8 fs with $2 \mathrm{~mJ}$ pulse energy has been observed by Stibenz et al. ${ }^{40}$ and self-compression of MIR pulses to few-cycle pulse duration, 32 fs at $3.2 \mu \mathrm{m}$ central wavelength with $3 \mu \mathrm{J}$ pulse energy, has been demonstrated by Hemmer et al. ${ }^{42}$

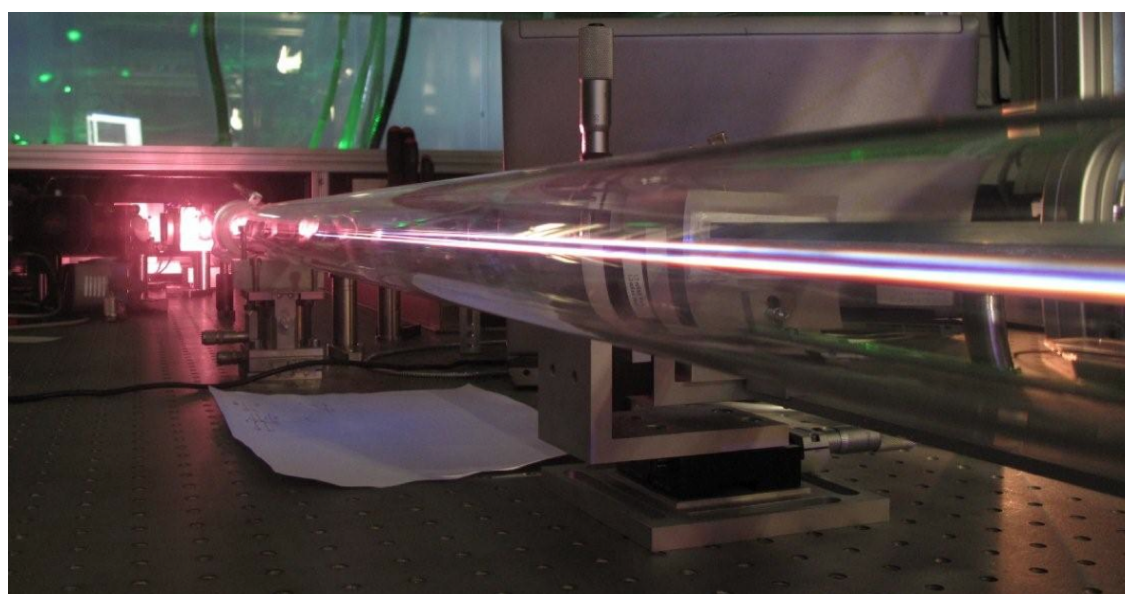

Figure 2.4: Picture of a filament taken in our lab

\section{Intense few-cycle pulses: State of the art}

Current lasers delivering high-energy ultrashort light pulses down to few optical cycles play a key role in contemporary ultrafast science. ${ }^{1,43-45}$ Such pulses have very broad spectra, well beyond the gain bandwidth of conventional amplifying media such as Ti:sapphire. ${ }^{46}$ Although Ti:sapphire amplifiers can easily lift the pulse energy to several Joules, the pulse durations don't fall below 15 fs. Therefore these lasers are followed by an external compression stage where the spectrum of the amplified pulses is broadened most frequently via SPM. To date, there are several compression schemes that lead to pulse durations down to the few-cycle regime. Traditionally the hollow core fibre is the most common pulse compression scheme used for extreme pulse compression below $5 \mathrm{fs}^{5,24}$ The main advantage of using hollow fibres is the excellent beam quality ${ }^{34}$ and spectral homogeneity across the beam profile. Ionization and material damage 
limit the output energy of the hollow fibre scheme to sub-milijoule levels, which can be extended to few milijoules using increasing pressure gradients along the hollow fibre $29,34,47$ or the use of circular polarization. ${ }^{48,49}$ Self-guided propagation in filaments ${ }^{6,50}$ is another common pulse compression technique. Advantages are a simple experimental setup and that self-compression can be achieved under certain circumstances. ${ }^{40}$ The pulse energies after the filament are limited to a few milijoules due to the appearance of multiple filamentation and intensity clamping at increased input energies. Wang and co-workers presented a compression scheme based on cascading filamentation and a hollow fibre. ${ }^{51}$ Combining the advantages of these two techniques has the potential of supporting sub4 fs pulse compression. More recently Nurhuda et al. proposed a pulse compression scheme based on self-phase modulation in a gas-filled planar waveguide (PWG) ${ }^{52}$ Because the input laser beam is confined only in the lateral direction perpendicular to the waveguide plane, thus enlarging the mode area, few cycle pulses with much higher energies can be generated. ${ }^{8-10}$ In the former scheme spatial beam quality still remains a problem. A summary of the most important laser sources delivering intense few-cycle pulses in the NIR is listed in table 2.1.

\subsubsection{Nonlinear pulse compression}

Although there is a nonlinear interaction involved when shortening pulses by means of the "classical" approach, the final compression of the temporal chirped and spectrally broadened pulse remains linear. A completely different compression scheme, called nonlinear pulse compression ${ }^{4}$ was proposed at the start of the 1990s by Wang and Dragila ${ }^{11}$ and Stabinis et al. $^{12}$ The term nonlinear pulse compression (NPC) refers to a pulse compression mechanism based on nonlinear frequency upconversion of light pulses in nonlinear crystals in the case of strong energy exchange and particular conditions of group-velocity mismatch. Thereto the crystal has to be chosen such that the group velocities $v_{1}$ and $v_{2}$ of the appropriately delayed pump pulses and $v_{S F}$ of the sum-frequency (SF) generated pulse meet the following condition:

$$
v_{1}<v_{S F}<v_{2}
$$

In other words, in a frame of reference moving at the velocity $v_{S F}$, the delayed pump pulses with the group velocities $v_{1}$ and $v_{2}$ will travel towards

\footnotetext{
${ }^{4}$ Sometimes referred to as second harmonic pulse compression or sum-frequency pulse compression.
} 
2.1. Optical pulse compression

\begin{tabular}{|c|c|c|}
\hline Reference & FWHM / Energy & Dispersive Delay line \\
\hline \multicolumn{3}{|c|}{ Filamentation } \\
\hline Hauri et al. (2004) & $5.7 \mathrm{fs} / 380 \mu \mathrm{J}$ & Chirped mirrors \\
\hline Guandalini et al. $(2006)^{53}$ & $5.1 \mathrm{fs} / 180 \mu \mathrm{J}$ & Chirped mirrors \\
\hline Stibenz et al. $(2006)^{40}$ & $7.8 \mathrm{fs} / 2000 \mu \mathrm{J}$ & Self-compression \\
\hline Chen et al. $(2007)^{41}$ & $5.0 \mathrm{fs} / 700 \mu \mathrm{J}$ & Chirped mirrors \\
\hline Park et al. $(2008)^{54}$ & $5.5 \mathrm{fs} / 500 \mu \mathrm{J}$ & Chirped mirrors \\
\hline \multicolumn{3}{|c|}{ Gas-filled hollow fibres } \\
\hline Nisoli et al. (1997) ${ }^{5}$ & $5.3 \mathrm{fs} / 70 \mu \mathrm{J}$ & Prisms and chirped mirrors \\
\hline Sansone et al. $(2003)^{55}$ & $4.6 \mathrm{fs} / 20 \mu \mathrm{J}$ & Chirped mirrors \\
\hline Schenkel et al. $(2003)^{27}$ & $3.8 \mathrm{fs} / 15 \mu \mathrm{J}$ & Chirped mirrors \\
\hline Suda et al. $(2005)^{29}$ & $9.8 \mathrm{fs} / 5 \mathrm{~mJ}$ & Chirped mirrors \\
\hline Oishi et al. $(2005)^{30}$ & $9.0 \mathrm{fs} / 2.5 \mathrm{~mJ}$ & Chirped mirrors \\
\hline Sung et al. $(2006)^{32}$ & $5.5 \mathrm{fs} / 1.1 \mathrm{~mJ}$ & Chirped mirrors \\
\hline Bohman et al. $(2008)^{34}$ & $5.4 \mathrm{fs} / 2.7 \mathrm{~mJ}$ & Chirped mirrors \\
\hline Park et al. (2009) ${ }^{33}$ & $3.7 \mathrm{fs} / 1.2 \mathrm{~mJ}$ & Chirped mirrors \\
\hline Bohman et al. $(2010)^{7}$ & $5.0 \mathrm{fs} / 5 \mathrm{~mJ}$ & Chirped mirrors \\
\hline Chen et al. $(2011)^{49}$ & $4.8 \mathrm{fs} / 1.6 \mathrm{~mJ}$ & Chirped mirrors \\
\hline Anderson et al. $(2011)^{56}$ & $5.7 \mathrm{fs} / 1.9 \mathrm{~mJ}$ & Chirped mirrors \\
\hline \multicolumn{3}{|c|}{ Cascaded filamentation and hollow fibres } \\
\hline Wang et al. $(2008)^{51}$ & $4.6 \mathrm{fs} / 200 \mu \mathrm{J}$ & Chirped mirrors \\
\hline \multicolumn{3}{|c|}{ Planar wave quides } \\
\hline Chen et al. (2008) ${ }^{9}$ & $12 \mathrm{fs} / 2 \mathrm{~mJ}$ & Chirped mirrors \\
\hline Akturk et al. $(2009)^{8}$ & $11.5 \mathrm{fs} / 7.6 \mathrm{~mJ}$ & Chirped mirrors \\
\hline Chen et al. $(2011)^{10}$ & $9.4 \mathrm{fs} / 9.1 \mathrm{~mJ}$ & Chirped mirrors \\
\hline
\end{tabular}

Table 2.1: High energy few-cycle pulses: State of the art.

each other (see Fig. 2.5). The shortening of the upconverted pulse originates from the narrow temporal overlap region of the pump pulses. When operating in the nonlinear regime both pump waves experience depletion, i.e. the leading edge of the faster pump pulse and the trailing edge of the slower one are depleted. In this way the area of pulse temporal overlap does not broaden. The saturation of the SF generated wave and resulting pulse temporal reshaping (amplitude modulation) of the pump pulses provide the excess bandwidth over the phasematching bandwidth. All parameters have to be accurately matched, since it is also possible to observe bandwidth narrowing at high-intensity conversion. ${ }^{57}$ 

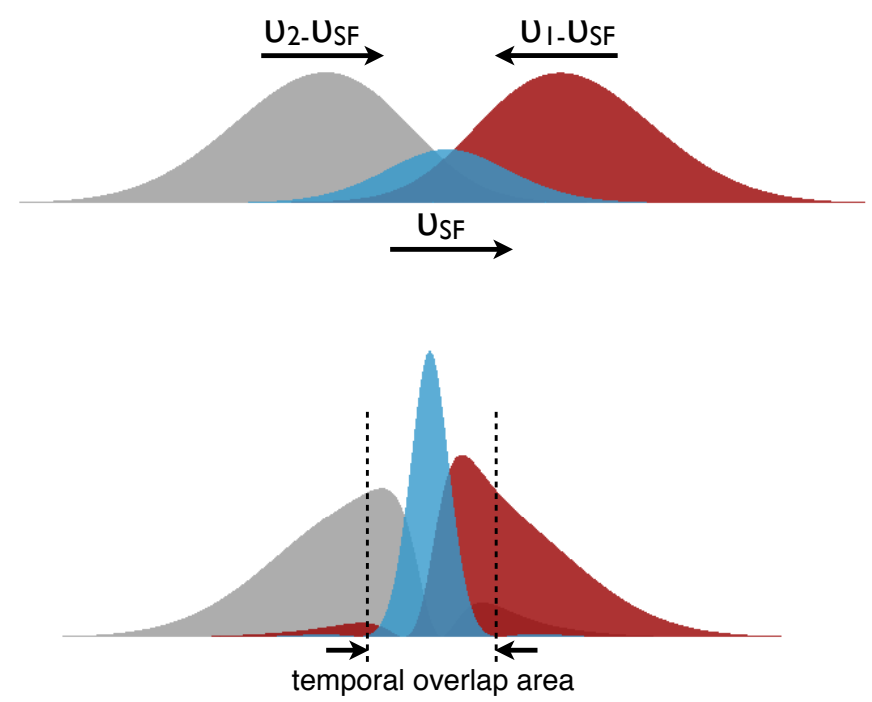

Figure 2.5: Nonlinear three pulse interaction under large group-velocity mismatch and strong energy exchange. The leading edge of the faster (grey) and the trailing edge of the slower pump pulse (red) are depleted. As a consequence the temporal overlap area is reduced and a very short $\mathrm{SF}$ pulse (blue) is generated.

Nonlinear pulse compression was first demonstrated in potassium dihydrogen phosphate (KDP) crystals. ${ }^{58}$ This type II phasematching crystal has an almost perfect set of dispersion parameters for the compression of picosecond pulses at around $1.06 \mu \mathrm{m}$, e.g., Nd-doped lasers. Using the group velocity mismatch between two orthogonally polarized pump pulses, compression factors of 4-7 were achieved starting with 0.5-1.2 ps pulses from $\mathrm{Nd}$ :glass lasers ${ }^{58-60}$ and compression factors in excess of 30 have been observed starting with 10-15 ps from Nd:YAG lasers. ${ }^{61,62}$

As mentioned above for pump pulses around $1 \mu \mathrm{m}, \mathrm{KDP}$ and deuterated $K D^{*} P$ are very suitable crystals for nonlinear pulse compression. However, finding alternative crystals with the required dispersion parameters, Eq. 2.4, at different wavelengths is quite challenging. Nisoli et al. generated pulses as short as 34 fs by SF pulse compression in a $\beta$-Barium Borate (BBO) crystal with type II phasematching of $1.3 \mu \mathrm{m}$ and $1.95 \mu \mathrm{m},{ }^{63}$ thus matching the interacting wavelengths to the group-velocity mismatch of a BBO crystal. In order to extend the appropriate GVD parameters of a nonlinear crystal to particular wavelengths, one can apply pulse-front tilts to modify the effective group velocities. That way, Dubietis et al. achieved 
ninefold nonlinear compression of the $\mathrm{SH}$ pulses of a $\mathrm{Nd}$ :glass laser in a BBO type II crystal. ${ }^{64}$ They used a collinear setup similar to the ones used for achromatic phasematching for $\mathrm{SH}$ generation of femtosecond pulses. ${ }^{65}$ A drawback of this method is that the angular dispersion which is imposed on the compressed pulse needs to be compensated afterwards. A better approach is to employ a noncollinear geometry, as proposed by Zhang et al., ${ }^{66}$ to meet the conditions required for nonlinear pulse compression. The concept of angle tuned phasematching already found successful applications in travelling-wave optical parametric generators, ${ }^{67}$ optical parametric oscillators ${ }^{68}$ and optical parametric amplifiers. ${ }^{69-71}$ Applied to the field of nonlinear pulse compression, the generation of $2.5 \mathrm{fs} \mathrm{SH}$ pulses should be feasible in a type II BBO using input pulse with 10 fs duration at $800 \mathrm{~nm} .{ }^{72}$ Although pre-compensation of the pulse fronts is needed in a noncollinear setup, the involved angles of pulse-front tilts are much smaller than a collinear three-wave interaction with tilted input pulses and the upconverted pulse doesn't end up tilted. The physics behind this process will be discussed in Chap. 3.

An interesting feature of the NPC process is that not only the upconverted pulse is compressed but also the pump pulses can undergo a notable shortening. By unbalancing the intensities of the fundamental frequency pulses, one can control the energy exchange between the interacting pulses. ${ }^{73}$ If started at the proper time, part of the short SF pulse is back-converted to the pump pulse and the subsequent pulse reshaping results in compression. The underlying effect can be attributed to the cascading of second-order processes under exact phasematching conditions. ${ }^{74}$

Nonlinear pulse compression using sum-frequency mixing is free of aperture limitations since the intensities required are well below the beam self-focusing threshold. Another advantage of this method is that no additional GVD components are required, which makes it feasible to compress bandwidth-limited pulses in a single step.

\subsection{Ultrashort UV pulses}

Ultrashort ultraviolet (UV) laser sources are important tools for a wide range of applications in femtochemistry, nonlinear optics, material science and attoscience. Unfortunately, robust, simple, and reliable laser sources that directly generate ultrashort UV pulses are not yet available, but extremely short optical pulses have been demonstrated throughout the visible and in the near-infrared ${ }^{44,46,75,76}$ (see also section 2.1.2). To generate ultrashort 
pulses in the UV longer wavelength lasers are used, i.e. mostly Ti:sapphire systems relying on a range of processes based on higher order nonlinearities $\left(\chi^{(2)}\right.$ and $\left.\chi^{(3)}\right)$. The first part of this section introduces a variety of laser systems delivering pulses in the visible and in the UV region. The second part describes several experiments conducted with UV radiation.

\subsubsection{Ultrafast UV sources}

There is an increased interest of the ultrafast laser community to have laser systems at various wavelengths with ultrashort pulses and high energies for daily applications. This section provides a concise overview of laser systems generating ultrashort pulses below and above $400 \mathrm{~nm}$, the second harmonic wavelength of a Ti:sapphire laser, and highlights the sources delivering sub $10 \mathrm{fs}$ pulses at this wavelength.

\section{From the visible to the deep ultraviolet}

The visible spectrum is well covered by noncollinear optical parametric amplifiers (NOPAs). Sub-10-fs pulses that are tunable across the visible spectrum can be obtained by ultra-broadband amplification of the visible portion of a white-light continuum in a BBO optical parameter amplifier pumped by the second harmonic of a Ti:sapphire laser. The large amplification bandwidth is made possible by a noncollinear phasematching scheme. NOPAs typically operate with a relatively short interaction length, short pump pulses and have the potential of generating few-cycle pulse. ${ }^{69-71}$

To go below $400 \mathrm{~nm}$, several successful methods for generating $10 \mathrm{fs}$ deep ultraviolet (DUV) pulses have been proposed so far. One is achromatic frequency doubling of visible ultrashort pulses from a noncollinear optical parametric amplifier. Sub 10 fs pulses in the region of 275-335 nm were generated with an energy of a few hundred nanojoule per pulse. ${ }^{77}$ However, absorption by nonlinear crystals cannot be avoided in the shorter wavelength region, which makes $\sim 250 \mathrm{~nm}$ to be the shortest wavelength to be achieved by this method. To overcome this issue noble gases, which are transparent in the DUV region, were used as nonlinear optical media in the following frequency conversion methods. With a non-degenerate four-wave mixing process, $2 \omega+2 \omega-\omega \rightarrow 3 \omega$, one can generate ultrashort DUV pulses from a powerful Ti:sapphire laser combined with its second harmonic. Due to the low nonlinear susceptibility of the gas media, a long interaction length is necessary, i.e. hollow waveguides are often used. Generation of sub-10 fs pulses in the DUV region was reported for this technique. ${ }^{78-81}$ Using filamentation instead of a hollow waveguide led 
to the generation of $12 \mathrm{fs}$ pulses centred at $260 \mathrm{~nm}$ with $10 \mu \mathrm{J}$ pulse energies. $^{82}$ Graf et al. proposed the generation of harmonics in a gas jet instead of crystals and demonstrated the generation of 4 fs pulses with this method; 83 they produced $1.4 \mu \mathrm{J}$ pulses at around $275 \mathrm{~nm}$ but required a regeneratively amplified $6 \mathrm{fs}, 0.25 \mathrm{~mJ}$ Ti:sapphire laser. Using a gas cell instead of a gas jet, Kosma et al. generated $11 \mathrm{fs}$ pulses at $160 \mathrm{~nm}^{84}$ and recently Reiter et al. generated sub 3 fs pulses centred at $260 \mathrm{~nm}$, starting with 4 fs pulses in the NIR. ${ }^{85}$

Another approach is to use a dielectric mirror to select the self compressed UV part of a supercontinuum generated in Argon with $12 \mathrm{fs}$ pulses centred at $800 \mathrm{~nm}^{86}$

\section{Sub 10 fs around $400 \mathrm{~nm}$}

Surprisingly there exist few ultrafast laser systems delivering sub 10 fs at the $\mathrm{SH}$ wavelength of Ti:sapphire, more precisely: only four systems have been reported to date. ${ }^{4,87-89}$

The most direct approach appears to be frequency doubling of an ultrashort NIR pulse, but for type I broadband phasematching very thin crystals have to be used in order to avoid spectral narrowing of the second harmonic by group-velocity mismatch, which would result in temporal pulse broadening. Yet, the conversion efficiency of the $\mathrm{SH}$ decreases as the crystal's thickness decreases. To overcome the spectral narrowing, one can use a broadband frequency doubling (BFD) scheme. Using $15 \mathrm{fs}$ pulse at $800 \mathrm{~nm}$ from a Ti:sapphire laser with an adaptive phase controller, Zhou et al. generated $7.5 \mathrm{fs}, 200 \mu \mathrm{J}$ pulses ${ }^{4}$ that way. However, a sub-15 fs pulse is not easily to be obtained directly from a Ti:sapphire amplifier system and the pulse front distortion due to a single-lens BFD setup must be compensated thoroughly. ${ }^{90}$ All in all a very complex setup.

Two laser systems are based on hollow core fibre compression of frequency doubled NIR pulses. The mechanism behind this method is the same as explained in Chap. 2 applied to the UV. In 1999 Dühr et al. demonstrated the generation of $8 \mathrm{fs}, 15 \mu \mathrm{J}$ pulses ${ }^{87}$ this way. Nearly ten years later with a very similar setup $7.5 \mathrm{fs}, 4 \mu \mathrm{J}$ pulses were generated by Liu et al. ${ }^{88}$

The last method is based on impulsive excitation ${ }^{91}$ of molecular motion in a hollow fibre filled with a Raman-active gas, e.g., SF $_{6}$. First a short and high intensity pulse, $30 \mathrm{fs}$ long, $0.5 \mathrm{~mJ}$ pulse of a Ti:sapphire laser system, excites impulsively a coherent molecular motion (vibration or rotation) in a molecular gas and a second, relatively weak delayed pulse, 27 fs long, $10 \mu \mathrm{J}$ pulses at $400 \mathrm{~nm}$, obtains a strong phase modulation due to refractive index variation caused by this molecular motion. As a result sub 4 fs 
pulses with energies of $1.5 \mu \mathrm{J}$ at $400 \mathrm{~nm}$ were generated. ${ }^{89}$

In the context of nonlinear pulse compression Biegert et al. ${ }^{72}$ theoretically demonstrated a technique which has the potential of generating directly sub $10 \mathrm{fs}$ pulses around $400 \mathrm{~nm}$ starting with pulses ranging from 10 to $50 \mathrm{fs}$ centred at $800 \mathrm{~nm}$.

The methods outlined above, except the first one which relies on a unique laser system, use a waveguide which imposes a limit on the aperture and thus on the power of the compressed pulse. The NPC method is free of aperture limitations; hence, it should be possible to generate much higher-energy pulses.

\subsubsection{UV applications}

Of all the conceivable applications using femtosecond pulses in the ultraviolet, filamentation, femtochemistry and coherent XUV generation are the most promising ones, which will be explained in this section.

\section{Filamentation at $400 \mathrm{~nm}$}

Nonlinear propagation of ultrashort laser pulses in various transparent media such as gases, transparent solids and liquids has been a subject of great interest in recent years. Most experimental and theoretical studies on filamentation have been performed by using femtosecond pulses in the near-infrared resulting in various technologically important applications including few-cycle optical pulse generation, ${ }^{6}$ terahertz generation, ${ }^{92}$ and remote sensing. ${ }^{93}$ Nevertheless pulses in the UV have several characteristics, which are beneficial for the generation of filaments and have attracted several research groups. Already in 1995, some authors pointed out that by using UV pulses, one should obtain continuous ionization channels over one kilometre length: ${ }^{94}$ The ionization rate of air is much more significant in the UV domain because it requires the simultaneous absorption of 3-4 photons to ionize an oxygen molecule, instead of 8-10 photons at $800 \mathrm{~nm}$ and the critical power for self-focusing is one order of magnitude lower at $400 \mathrm{~nm}$. Thus, the filamentation is achieved much easier using ultraviolet pulses and it should be possible to generate high quality and long distance filaments. So far $70 \mathrm{~m}$ long filaments could be generated with femtosecond laser pulses centred at $400 \mathrm{~nm}$ in air. ${ }^{95}$ In general, subpicosecond filament propagation of UV pulses in air has been studied extensively at the start of the 2000s. ${ }^{96-99}$ Béjot et al. showed that the spectral broadening can be enhanced over two octaves and that a connected plasma more than 3 times longer than the initial ones can be formed by two-colour co-filamentation 
of ultrashort laser pulses at $400 \mathrm{~nm}$ and $800 \mathrm{~nm}$ in argon. ${ }^{100}$ The resulting ultra-broadband pulses could be used for the coherent control of states spanning over a large energy interval. ${ }^{101}$

\section{Femtosecond spectroscopy}

Femtosecond spectroscopy is a very powerful technique making it possible to follow dynamic processes in materials or chemical compounds on ultrashort time scales in real time. Experiments are based on pump-probe techniques, where the sample is excited by an ultrashort pump pulse and a second time-delayed probe pulse probes the changes of the optical properties of the sample. By applying suitable time delays one obtains the information of the monitored properties, e.g., reflectivity, absorption, fluorescence, Raman scattering, on a femtosecond time scale. The time resolution of such spectroscopic techniques is limited by the pulse durations of the pump and probe pulses. Using femtosecond lasers, ultrafast phenomena such as phonon and vibrational dynamics could be studied with a time resolution of a few femtoseconds. ${ }^{102-104}$ Most of the experiments using sub 10 fs pulses were performed in the visible and NIR region due to lack of ultrafast laser sources in the UV. However, extension of sub-10 fs spectroscopy to the DUV region would be beneficial as many basic aromatic molecules have electronic transitions in this wavelength region. ${ }^{105}$ One of the most prominent molecules that absorb DUV radiation are DNA and amino acids. Recently experiments applying transient absorption spectroscopy with sub 10 fs DUV on thymine were reported for the first time. ${ }^{106}$

\section{XUV generation}

The extreme ultraviolet (XUV) wavelength range is very attractive for many research areas such as nanoscience, chemistry, physics, and biology. An important and widely used method for generating coherent XUV and soft$\mathrm{x}$-ray radiation is high-order harmonic generation (HHG). HHG combines spatial resolution of tens of nanometres and temporal accuracy of tens of attoseconds in the form of attosecond pulses. The mechanism behind HHG can be described with the semi-classical three-step model, which includes ionization, electron propagation, and recombination. ${ }^{107,108}$ It is well known from the three-step model that the cutoff energy (maximum phonon energy), $E_{\text {cutoff }}$, scales as described by the following equation:

$$
E_{\text {cutoff }}=I_{p}+3.17 \frac{E_{0}^{2} e^{2}}{4 m \omega_{0}^{2}},
$$


where $I_{p}$ is the ionization potential, $e$ and $m$ are the electron charge and mass, and $E_{0}$ and $\omega_{0}$ are the driver field amplitude and frequency. Equation 2.5 indicates that the cutoff photon energy can be extended by using long-wavelength driver pulses and maximizing the electric field amplitude. ${ }^{109-112}$ Recently, bright high-harmonic $x$-ray supercontinua with photon energies spanning from the XUV to $1.6 \mathrm{keV}$ were generated by focusing mid-IR $(3.9 \mu \mathrm{m})$ femtosecond pulses into a waveguide filled with Helium gas. ${ }^{113}$ However, the conversion efficiency of HHG decreases dramatically with longer-wavelength driving fields, with a very unfavourable $\lambda^{-4.8}-\lambda^{-6}$ scaling, depending on the intensity and choice of atom. ${ }^{110,114-116}$ Hence, if the final objective is to generate radiation in the range of $10-100 \mathrm{eV}$, the use of shorter wavelength driver pulses can improve the HHG efficiency by several orders of magnitude. Experimentally, conversion efficiencies exceeding $10^{-5}$ at $\sim 90 \mathrm{eV} \mathrm{HHG}$ with a $400 \mathrm{~nm}$ driving laser have been demonstrated. ${ }^{117}$ Compared to typical HHG efficiencies of $10^{-7}-10^{-8}$ achieved with $800 \mathrm{~nm}$ driving lasers, ${ }^{44}$ this is an improvement of two to three orders of magnitude. Important applications in the $<100 \mathrm{eV}$ range that require very high photon flux are XUV lithography, ${ }^{118-120}$ seeding of $\mathrm{X}$-ray freeelectron lasers, ${ }^{121-123}$ and attosecond spectroscopy. ${ }^{43,124-126}$ For these applications the efficient generation of XUV high harmonics driven by ultrashort $400 \mathrm{~nm}$ pulses, with slightly higher electric fields than that of $800 \mathrm{~nm}$ pulses, could have a significant impact.

\subsection{Ultrashort NIR to MIR pulses}

The development of ultrafast laser sources in the near-infrared (NIR) to mid-infrared (MIR) spectral region is attracting attention owing to applications in biology, in medicine ${ }^{127}$ and in high-intensity laser-matter interactions. ${ }^{115}$ However, a point to be considered is the lack of available gain media with the required bandwidth to support lasing and generation of ultrashort (less than $50 \mathrm{fs}$ ) pulses in the NIR to MIR range. For this reason ultrashort pulse generation schemes in this wavelength region are, to date, mostly based on optical parametric amplification (OPA) in crystals with second-order, $\chi^{2}$, nonlinearity.

\subsubsection{Ultrafast NIR to MIR sources}

While current ultrashort pulse sources are highly developed, their central wavelengths almost exclusively lie below $1 \mu \mathrm{m}$. The most common approach for accessing the NIR to MIR region proceeds via downconversion 
of NIR sources by difference-frequency generation (DFG), optical parametric generation (OPG), or optical parametric oscillation (OPO). ${ }^{128}$ DFG offers straightforward single-pass geometries relative to OPO and offers higher conversion efficiencies compared with OPG. In the context of fewcycle light pulse generation at longer wavelengths, probably the most established method consists of noncollinear OPA of some white light continuum or frequency-shifted output generated from a Ti:sapphire laser ${ }^{70}$ or, more recently, Yb-based fibre chirped pulse amplification (CPA) systems. ${ }^{129}$ For the ultra-broadband spectra DFG between different spectral components of a hollow-fibre broadened supercontinuum can also be used. ${ }^{130}$ Different implementations of these various approaches have generated few-cycle pulses at 1.2-3 $\mu \mathrm{m} .{ }^{130-133}$ For example, pulse energies up to $1 \mathrm{~mJ}$ and durations as short as $17 \mathrm{fs}$ at $1.4 \mu \mathrm{m}$ have been reported using a $60 \mathrm{fs}$ Ti:sapphire laser as a pump source. ${ }^{134}$

A traveling-wave optical parametric amplifier, when pumped by a femtosecond Ti:sapphire amplifier and seeded with $1 \mathrm{~ns}$ pulses around $1 \mu \mathrm{m}$ wavelength has been shown to produce tunable pulses between 3.1 and $3.9 \mu \mathrm{m} .{ }^{135}$ However such a system is limited to roughly the duration of the driving laser pulse and suffers from low efficiency.

Optical parametric chirped pulse amplification (OPCPA) ${ }^{136,137}$ is another promising route to ultrashort pulse generation in the NIR to MIR region as it supports extremely high repetition rates, is in principle carrierenvelope phase (CEP) preserving, and offers the possibility of direct amplification of ultra-broadband spectra across a huge range of central wavelengths in the near- and mid-infrared. In contrast to OPA based schemes, OPCPA involves the amplification of chirped seed pulses using a narrowband, typically pico- to nanosecond, pump laser. This approach allows the energy of the system to be scaled up even to joule level. ${ }^{138,139}$ Indeed, NIR OPCPA sources have demonstrated that they can directly produce amplified few-cycle pulses as short as $5.5 \mathrm{fs}^{140}$ and have already been reported at $2.1 \mu \mathrm{m}^{141}$ and in the mid-IR at $3.2 \mu \mathrm{m}^{142}$ The novel design of the latter OPCPA directly produces CEP stable pulses and allows self-compression to sub-3-cycle duration by nonlinear propagation in the anomalous dispersion regime in dielectric media. ${ }^{42}$

Another interesting approach to ultra-broadband MIR coherent radiation uses four-wave mixing through filamentation in air. Pulses as short as 13 fs with an octave-spanning spectrum reaching from 2.5-5.5 $\mu \mathrm{m}$ have been generated without any compressors by focusing the $800 \mathrm{~nm}$ fundamental of a Ti:sapphire laser system and its second harmonic in air. ${ }^{82}$ Using a very similar approach, i.e. argon instead of air, Faccio et al. ${ }^{143}$ gen- 
erated pulses peaked at $1.4 \mu \mathrm{m}$ wavelength, with $5 \mu \mathrm{J}$ pulse energy and $45 \mathrm{fs}$ duration. The output energy of more than a few microjoule is still sufficient for spectroscopy. Note that energy scaling is inherently limited to microjoule energies due to intensity clamping in the filament using this method.

\subsubsection{NIR to MIR applications}

Ultrashort pulses in the NIR to MIR region are of great interest for use in numerous applications, specifically in biomedical science and in strongfield science.

While visible wavelengths are more suitable for imaging applications, due to a longer penetration depth, NIR to MIR wavelengths hold a clear advantage in terms of selectivity and rapid (and localized) absorption. This is especially beneficial for single-cell laser nanosurgery ${ }^{144}$ which is used in an increasing range of methodologies. Its main advantages are high spatial selectivity, high accuracy in time, there is not any physical contact with the cell so they remain in a sterile environment, and high throughput compared to micromanipulators.

The development of ultrashort pulsed lasers in the near- to mid-infrared spectral region is highly important for studies on molecular vibrational dynamics, since the vast majority of gaseous chemical substances exhibit fundamental vibrational absorption bands between 2 and $25 \mu \mathrm{m}$. Applications range from the identification of biomarker molecules ${ }^{145}$ which have been correlated to specific diseases and metabolic processes., e.g., from the detection of lung cancer ${ }^{146}$ to monitoring the concentration of green house gases. ${ }^{147}$

In strong-field physics, when the laser electric field strength approaches that of the atomic binding energy, intense, ultrafast laser sources in the NIR to MIR region have considerable advantages. Many strong-field physics experiments involve the measurement of photoionised electrons. Current ultrashort laser sources operating around $800 \mathrm{~nm}$ introduce experimental ambiguities due to a mixture of tunnelling and multi-photon ionisation, whereas longer wavelength pulses allow a much clearer discrimination. The lower photon energy ensures tunnelling ionisation only, allowing investigation of strong-field atomic processes with unprecedented clarity. In high harmonic generation an important limit is the maximum photon energy that can be generated by the driving laser. Due to a square of wavelength dependence of the shortest wavelength reachable via high harmonic generation, Eq. 2.5 , the cutoff energy can be substantially extended using 
longer wavelength lasers, ${ }^{148,149}$ allowing the generation of even shorter single attosecond pulses. 
CHAPTER 2. ULTRASHORT PULSES 
In this chapter the concepts of nonlinear interaction of light and matter will be introduced via the nonlinear polarization, which will be used together with Maxwells equations to derive the equations describing the relationship between NIR waves and their sum-frequency generation (SFG). Finally the coupled wave equations will be used to derive equations governing gain in BBO. In section 3.2 the phasematching conditions are studied. This conditions are depending on the three interacting wavevectors and further on the crystal cut angle, the noncollinear angles and the angular dispersion. 


\section{Chapter 3}

\section{Theory of nonlinear three-wave interaction}

\subsection{The coupled wave equations}

An important consequence of the nonlinearity of the susceptibility is the mixing of different frequencies, e.g., sum-frequency mixing. Let us consider the combination of two monochromatic fields with different frequencies,

$$
\mathbf{E}(x, t)=\frac{1}{2}\left[\tilde{\mathbf{E}}\left(x, \omega_{1}\right) e^{j \omega_{1} t}+\tilde{\mathbf{E}}\left(x, \omega_{2}\right) e^{j \omega_{2} t}+\text { c.c. }\right],
$$

where $\mathbf{E}(x, t)$ is the electric field as a function of position $x$ and time $t, \omega_{i}$ is the angular frequency and c.c. stands for conjugate complex. The location independent term $\tilde{\mathbf{E}}(x, \omega)$ fulfils the Helmholtz equation,

$$
\nabla^{2} \tilde{\mathbf{E}}(x, \omega)+\frac{\omega^{2} \epsilon}{c_{0}^{2}} \tilde{\mathbf{E}}(x, \omega)=0,
$$

where $\epsilon$ is the permittivity and $c_{0}$ is the speed of light. Thus, in a Cartesian coordinate system each field component $\tilde{\mathbf{E}}_{i}$ has to fulfil the scalar Helmholtz equation,

$$
\left[\frac{\partial^{2}}{\partial x^{2}}+\frac{\partial^{2}}{\partial y^{2}}+\frac{\partial^{2}}{\partial z^{2}}+\frac{\omega^{2} \epsilon}{c_{0}^{2}}\right] \tilde{\mathbf{E}}_{i}(x, \omega)=0 .
$$

It is relatively easy to show that one simple harmonic solution to the Helmholtz equation will take the form

$$
\tilde{\mathbf{E}}(x, \omega)=\tilde{\mathbf{E}}(k, \omega) e^{-j k x},
$$


where we have introduced the wavevector $k$,

$$
k=\frac{n \omega}{c_{0}}
$$

$n$ being the refractive index. The electric field can then be written as

$$
\begin{aligned}
\mathbf{E}(x, t) & =\frac{1}{2}\left[\tilde{\mathbf{E}}(\mathbf{k}, \omega) e^{-j(k x-\omega t)}+c . c .\right] \\
& =\operatorname{Re}[\tilde{\mathbf{E}}(x, t)]
\end{aligned}
$$

with $\tilde{\mathbf{E}}(x, t)=\tilde{\mathbf{E}}(\mathbf{k}, \omega) e^{-j(k x-\omega t)}$, the complex wavefunction, and $\tilde{\mathbf{E}}(\mathbf{k}, \omega)$, the complex amplitude.

Nonlinear optical phenomena are often described by a Taylor series expansion of the dielectric polarization density, the nonlinear polarization $\mathbf{P}$, in terms of electric fields,

$$
\mathbf{P}=\sum_{i} \mathbf{P}^{(i)}=\epsilon_{0} \chi^{(1)} \mathbf{E}+\epsilon_{0} \chi^{(2)} \mathbf{E E}+\epsilon_{0} \chi^{(3)} \mathbf{E E E}+\ldots
$$

Here, the coefficients $\chi^{(n)}$ are the $n$-th order susceptibilities of the medium and the presence of such a term is generally referred to as $n$-th order nonlinearity. If one substitutes now Eq. 3.1 into the quadratic term of the nonlinear polarization Eq. 3.7, one obtains

$$
\begin{aligned}
\mathbf{P}^{(2)}(x, t) & =\epsilon_{0} \chi^{(2)} \mathbf{E}(x, t) \mathbf{E}(x, t) \\
& =\epsilon_{0} \frac{1}{4} \chi^{(2)}\left[\tilde{\mathbf{E}}\left(x, \omega_{1}\right) \tilde{\mathbf{E}}\left(x, \omega_{1}\right) e^{j 2 \omega_{1} t}\right. \\
& +\tilde{\mathbf{E}}\left(x, \omega_{2}\right) \tilde{\mathbf{E}}\left(x, \omega_{2}\right) e^{j 2 \omega_{2} t} \\
& +2 \tilde{\mathbf{E}}\left(x, \omega_{1}\right) \tilde{\mathbf{E}}\left(x, \omega_{2}\right) e^{j\left(\omega_{1}+\omega_{2}\right) t} \\
& +2 \tilde{\mathbf{E}}\left(x, \omega_{1}\right) \tilde{\mathbf{E}}^{*}\left(x, \omega_{2}\right) e^{j\left(\omega_{1}-\omega_{2}\right) t} \\
& +\tilde{\mathbf{E}}\left(x, \omega_{1}\right) \tilde{\mathbf{E}}^{*}\left(x, \omega_{1}\right) \\
& \left.+\tilde{\mathbf{E}}\left(x, \omega_{2}\right) \tilde{\mathbf{E}}^{*}\left(x, \omega_{2}\right)\right] \\
& + \text { c.c. }
\end{aligned}
$$


which can be written in the form

$$
\begin{aligned}
\mathbf{P}^{(2)}(x, t) & =\frac{1}{2}\left[\tilde{\mathbf{P}}\left(x, 2 \omega_{1}\right) e^{j 2 \omega_{1} t}+c . c .\right] \\
& +\frac{1}{2}\left[\tilde{\mathbf{P}}\left(x, 2 \omega_{2}\right) e^{j 2 \omega_{2} t}+c . c .\right] \\
& +\frac{1}{2}\left[\tilde{\mathbf{P}}\left(x, \omega_{1}+\omega_{2}\right) e^{j\left(\omega_{1}+\omega 2\right) t}+\text { c.c. }\right] \\
& +\frac{1}{2}\left[\tilde{\mathbf{P}}\left(x, \omega_{1}-\omega_{2}\right) e^{j\left(\omega_{1}-\omega 2\right) t}+\text { c.c. }\right] \\
& +\frac{1}{2}[\tilde{\mathbf{P}}(x, 0)+\text { c.c. }] .
\end{aligned}
$$

The nonlinear polarization term $\mathbf{P}^{(2)}$ involving the sum-frequency components $\omega_{1}+\omega_{2}$ is

$$
\tilde{\mathbf{P}}\left(x, \omega_{1}+\omega_{2}\right)=\epsilon_{0} \chi^{(2)} \tilde{\mathbf{E}}\left(x, \omega_{1}\right) \tilde{\mathbf{E}}\left(x, \omega_{2}\right)
$$

and the one involving the difference-frequency components $\omega_{1}-\omega_{2}$ is

$$
\tilde{\mathbf{P}}\left(x, \omega_{1}-\omega_{2}\right)=\epsilon_{0} \chi^{(2)} \tilde{\mathbf{E}}\left(x, \omega_{1}\right) \tilde{\mathbf{E}}^{*}\left(x, \omega_{2}\right) .
$$

Assuming plane waves $\tilde{\mathbf{E}}\left(x, \omega_{i}\right)=\tilde{\mathbf{E}}\left(\mathbf{k}, \omega_{i}\right) e^{-j k_{i} x}$ (Eq. 3.4), the driving field will have the form

$$
\mathbf{E}(x, t)=\frac{1}{2}\left[\tilde{\mathbf{E}}\left(\mathbf{k}_{1}, \omega_{1}\right) e^{-j\left(k_{1} x-\omega_{1} t\right)}+\tilde{\mathbf{E}}\left(\mathbf{k}_{2}, \omega_{2}\right) e^{-j\left(k_{2} x-\omega_{2} t\right)}+c . c .\right]
$$

so that one obtains for the sum-frequency component of the nonlinear polarization

$$
\mathbf{P}_{\omega_{1}+\omega_{2}}^{(2)}(x, t)=\frac{1}{2}\left[\epsilon_{0} \chi^{(2)} \tilde{\mathbf{E}}\left(\mathbf{k}_{1}, \omega_{1}\right) \tilde{\mathbf{E}}\left(\mathbf{k}_{2}, \omega_{2}\right) e^{-j\left[\left(k_{1}+k_{2}\right) x-\left(\omega_{1}+\omega_{2}\right) t\right]}+\text { c.c. }\right] .
$$

This expression can be considered as a plane polarization wave with the wavevector

$$
\mathbf{k}_{3}=\mathbf{k}_{1}+\mathbf{k}_{2}
$$

acting as a source term for the following electromagnetic wave:

$$
\mathbf{E}_{\omega_{3}}(x, t)=\frac{1}{2}\left[\tilde{\mathbf{E}}\left(\mathbf{k}_{3}, \omega_{3}\right) e^{-j\left(k_{3} x-\omega_{3} t\right)}+\text { c.c. }\right],
$$

where $\omega_{3}=\omega_{1}+\omega_{2}$. In order that the coupling of the polarization wave with the electromagnetic wave is efficient, the absolute value and the direction of the wavevectors in Eq. 3.14 have to match. This condition is the so called phasematching condition because it ensures that the polarization wave, as 
source of the electromagnetic wave, maintains a constant phase relation to the latter. This way the amplitude of the sum-frequency wave can increase while propagating through the nonlinear medium, i.e. the electromagnetic wave is driven phase-matched through the polarization along the propagation direction.

In the photon picture the sum-frequency generation corresponds to a three photon process, where two photons of energies $\hbar \omega_{1}$ and $\hbar \omega_{2}$ are destroyed and one photon of energy $\hbar \omega_{3}$ is generated (see Fig. 3.1). The phasematching condition can be interpreted in this picture as momentum conservation, since the momentum of a photon is given by $\hbar \mathbf{k}$.

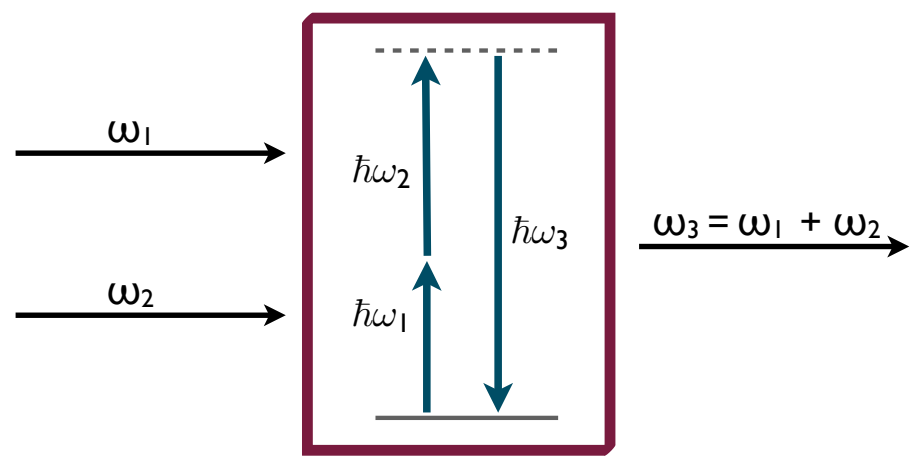

Figure 3.1: Sum-frequency generation in the photon picture.

\section{The coupled wave equations}

Starting with the most general form of the wave equation in nonlinear optics (Eq. 3.16), the coupled wave equations are derived for the special case of sum-frequency generation assuming a lossless nonlinear optical medium involving collimated, monochromatic, continuous-wave input beams.

The electromagnetic wave equation, derived from Maxwell's wave equations, in an isotropic, linear and local medium can be written as

$$
-\nabla^{2} \mathbf{E}+\mu_{o} \frac{\partial^{2} \mathbf{D}}{\partial t^{2}}=0
$$

where $\mu_{o}$ is the permeability and $\mathbf{D}$ is the electric displacement field. In order to analyse this frequency mixing process, one can substitute $\mathbf{D}=$ $\epsilon_{0} \mathbf{E}+\mathbf{P}, \mathbf{P}=\mathbf{P}^{(1)}+\mathbf{P}^{(2)}$ and $\epsilon_{0} \mathbf{E}+\mathbf{P}^{(1)}=\epsilon_{0} \epsilon \mathbf{E}$ leading to

$$
\nabla^{2} \mathbf{E}-\frac{\epsilon}{c_{0}^{2}} \frac{\partial^{2} \mathbf{E}}{\partial t^{2}}=\mu_{o} \frac{\partial^{2} \mathbf{P}^{(2)}}{\partial t^{2}} .
$$


Following Eq. 3.6, the three electric field components that are involved in the SFG process can be set as plane waves propagating in $z$-direction,

$$
\mathbf{E}_{i}(\mathbf{z}, t)=\frac{1}{2}\left[\tilde{\mathbf{E}}_{i}\left(\omega_{i}\right) e^{-j\left(k_{i} z-\omega_{i} t\right)}+\text { c.c. }\right], \quad i=1,2,3 .
$$

Those plane waves don't show a $x, y$-dependence, hereby the operator $\nabla^{2}$ in Eq. 3.17 is reduced to $\partial^{2} / \partial z^{2}$. If one takes into account that the complex amplitudes $\tilde{\mathbf{E}}_{i}\left(\omega_{i}\right)$ vary slowly in time, one can write the first term of Eq. 3.17 as follows,

$$
\begin{aligned}
\frac{\partial^{2}}{\partial z^{2}} \tilde{\mathbf{E}}_{i}\left(\omega_{i}\right) e^{-j\left(k_{i} z-\omega_{i} t\right)} & \\
= & {\left[\frac{\partial^{2}}{\partial z^{2}} \tilde{\mathbf{E}}_{i}\left(\omega_{i}\right)-2 j k_{i} \frac{\partial}{\partial z} \tilde{\mathbf{E}}_{i}\left(\omega_{i}\right)-k_{i}^{2} \tilde{\mathbf{E}}_{i}\left(\omega_{i}\right)\right] e^{-j\left(k_{i} z-\omega_{i} t\right)} } \\
& \approx \\
& {\left[-2 j k_{i} \frac{\partial}{\partial z} \tilde{\mathbf{E}}_{i}\left(\omega_{i}\right)-k_{i}^{2} \tilde{\mathbf{E}}_{i}\left(\omega_{i}\right)\right] e^{-j\left(k_{i} z-\omega_{i} t\right)} }
\end{aligned}
$$

With the fundamental dispersion relation, $k^{2}=\frac{\omega^{2}}{c_{0}^{2}} \epsilon$, the second term of Eq. 3.17 can be written as

$$
\begin{aligned}
-\frac{\epsilon_{\omega_{i}}}{c_{0}^{2}} \frac{\partial^{2}}{\partial t^{2}} \tilde{\mathbf{E}}_{i}\left(\omega_{i}\right) e^{-j\left(k_{i} z-\omega_{i} t\right)} & =\epsilon_{\omega_{i}} \frac{\omega_{i}^{2}}{c_{0}^{2}} \tilde{\mathbf{E}}_{i}\left(\omega_{i}\right) e^{-j\left(k_{i} z-\omega_{i} t\right)} \\
& =k_{\omega_{i}}^{2} \tilde{\mathbf{E}}_{i}\left(\omega_{i}\right) e^{-j\left(k_{i} z-\omega_{i} t\right)}
\end{aligned}
$$

In the case of difference-frequency generation (DFG), the right term of Eq. 3.17 involving the frequencies $\omega_{3}-\omega_{2}$ is

$$
\mu_{0} \frac{\partial^{2}}{\partial t^{2}} \tilde{\mathbf{P}}_{\left(\omega_{3}-\omega_{2}\right)} e^{-j\left(\left(k_{3}-k_{2}\right) z-\omega_{1} t\right)}=-\mu_{0} \omega_{1}^{2} \tilde{\mathbf{P}}_{\left(\omega_{3}-\omega_{2}\right)} e^{-j\left(\left(k_{3}-k_{2}\right) z-\omega_{1} t\right)},
$$

respectively for $\omega_{3}-\omega_{1}$

$$
\mu_{0} \frac{\partial^{2}}{\partial t^{2}} \tilde{\mathbf{P}}_{\left(\omega_{3}-\omega_{1}\right)} e^{-j\left(\left(k_{3}-k_{1}\right) z-\omega_{\mathbf{2}} t\right)}=-\mu_{0} \omega_{2}^{2} \tilde{\mathbf{P}}_{\left(\omega_{3}-\omega_{1}\right)} e^{-j\left(\left(k_{3}-k_{1}\right) z-\omega_{\mathbf{2}} t\right)} .
$$

In the case of the sum-frequency generation involving the frequencies $\omega_{1}+$ $\omega_{2}$ this term becomes

$$
\mu_{0} \frac{\partial^{2}}{\partial t^{2}} \tilde{\mathbf{P}}_{\left(\omega_{1}+\omega_{2}\right)} e^{-j\left(\left(k_{1}+k_{2}\right) z-\omega_{3} t\right)}=-\mu_{0} \omega_{3}^{2} \tilde{\mathbf{P}}_{\left(\omega_{1}+\omega_{2}\right)} e^{-j\left(\left(k_{1}+k_{2}\right) z-\omega_{3} t\right)}
$$

Substituting Eq. 3.19 and 3.20 into the wave equation Eq. 3.17 for the two cases of DFG (Eq. 3.21 and 3.22) and for the case of SFG (Eq. 3.23), one 
obtains

$$
\begin{aligned}
-2 j k_{1} \frac{\partial}{\partial z} \tilde{\mathbf{E}}\left(\omega_{1}\right) e^{-j k_{1} z} & =-\mu_{0} \omega_{1}^{2} \tilde{\mathbf{P}}_{\left(\omega_{3}-\omega_{2}\right)} e^{-j\left(k_{3}-k_{2}\right) z} \\
-2 j k_{2} \frac{\partial}{\partial z} \tilde{\mathbf{E}}\left(\omega_{2}\right) e^{-j k_{2} z} & =-\mu_{0} \omega_{2}^{2} \tilde{\mathbf{P}}_{\left(\omega_{3}-\omega_{1}\right)} e^{-j\left(k_{3}+k_{1}\right) z} \\
-2 j k_{3} \frac{\partial}{\partial z} \tilde{\mathbf{E}}\left(\omega_{3}\right) e^{-j k_{3} z} & =-\mu_{0} \omega_{3}^{2} \tilde{\mathbf{P}}_{\left(\omega_{1}+\omega_{2}\right)} e^{-j\left(k_{1}+k_{2}\right) z} .
\end{aligned}
$$

As described by Eq. 3.10 and Eq. 3.11 the nonlinear polarization terms can be written as

$$
\begin{aligned}
& \tilde{\mathbf{P}}_{\left(\omega_{3}-\omega_{2}\right)}=\epsilon_{0} \chi^{(2)} \tilde{\mathbf{E}}\left(\omega_{3}\right) \tilde{\mathbf{E}}^{*}\left(\omega_{2}\right) \\
& \tilde{\mathbf{P}}_{\left(\omega_{3}-\omega_{1}\right)}=\epsilon_{0} \chi^{(2)} \tilde{\mathbf{E}}\left(\omega_{3}\right) \tilde{\mathbf{E}}^{*}\left(\omega_{1}\right) \\
& \tilde{\mathbf{P}}_{\left(\omega_{1}+\omega_{2}\right)}=\epsilon_{0} \chi^{(2)} \tilde{\mathbf{E}}\left(\omega_{1}\right) \tilde{\mathbf{E}}\left(\omega_{2}\right) .
\end{aligned}
$$

Substituting Eq. 3.25 into Eq. 3.24 yields to the coupled-amplitude equations for the special case of sum-frequency generation,

$$
\begin{aligned}
\frac{d \tilde{\mathbf{E}}\left(\omega_{1}\right)}{d z} & =\frac{-j \omega_{1}}{2 c_{0} n_{\omega_{1}}} \chi^{(2)} \tilde{\mathbf{E}}\left(\omega_{3}\right) \tilde{\mathbf{E}}^{*}\left(\omega_{2}\right) e^{-j \Delta k z} \\
\frac{d \tilde{\mathbf{E}}\left(\omega_{2}\right)}{d z} & =\frac{-j \omega_{2}}{2 c_{0} n_{\omega_{2}}} \chi^{(2)} \tilde{\mathbf{E}}\left(\omega_{3}\right) \tilde{\mathbf{E}}^{*}\left(\omega_{1}\right) e^{-j \Delta k z} \\
\frac{d \tilde{\mathbf{E}}\left(\omega_{3}\right)}{d z} & =\frac{-j \omega_{3}}{2 c_{0} n_{\omega_{3}}} \chi^{(2)} \tilde{\mathbf{E}}\left(\omega_{1}\right) \tilde{\mathbf{E}}\left(\omega_{2}\right) e^{j \Delta k z}
\end{aligned}
$$

The quantity

$$
\Delta k=k_{3}-k_{2}-k_{1}
$$

is referred to as the wavevector mismatch.

Due to the reality of $\chi^{(2)}$ for a lossless medium, which was assumed for writing these equations in the forms shown, one can make use of the condition of full permutation symmetry

$$
\chi_{i j k}^{(2)}\left(\omega_{3}=\omega_{1}+\omega_{2}\right)=\chi_{j k i}^{(2)}\left(\omega_{1}=\omega_{3}-\omega_{2}\right)
$$

and

$$
\chi_{i j k}^{(2)}\left(\omega_{3}=\omega_{1}+\omega_{2}\right)=\chi_{k i j}^{(2)}\left(\omega_{2}=\omega_{3}-\omega_{1}\right)
$$

to conclude that the coupling coefficient $\chi^{(2)}$ has the same value in each equation. 


\subsection{Phasematching, group velocities and pulse-front tilts}

In this section the phasematching angles, the angular dispersion, and the GVD in a noncollinear arrangement inside a BBO crystal based on type II phasematching are investigated. Following the conditions (Eq. 2.4) necessary for pulse compression, the required pulse-front tilt (PFT) at the aircrystal interface and its pre-compensation are discussed.

\subsubsection{Phasematching}

In order to find out the exact phasematching angles inside the crystal, the condition for momentum conservation of the wavevectors

$$
\mathbf{k}_{e(\omega)}+\mathbf{k}_{o(\omega)}=\mathbf{k}_{e(2 \omega)},
$$

must be fulfilled. Equation 3.30 is solved at the centre frequency of a broadband NIR spectrum, where $\mathbf{k}_{e(\omega)}, \mathbf{k}_{o(\omega)}$ are respectively the wavevectors corresponding to the extraordinary, ordinary wave of the fundamentals, and $\mathbf{k}_{e(2 \omega)}$ corresponds to the extraordinary wave of the sum-frequency generated signal (see Fig. 3.2). Equation 3.30 can be solved for the trans-

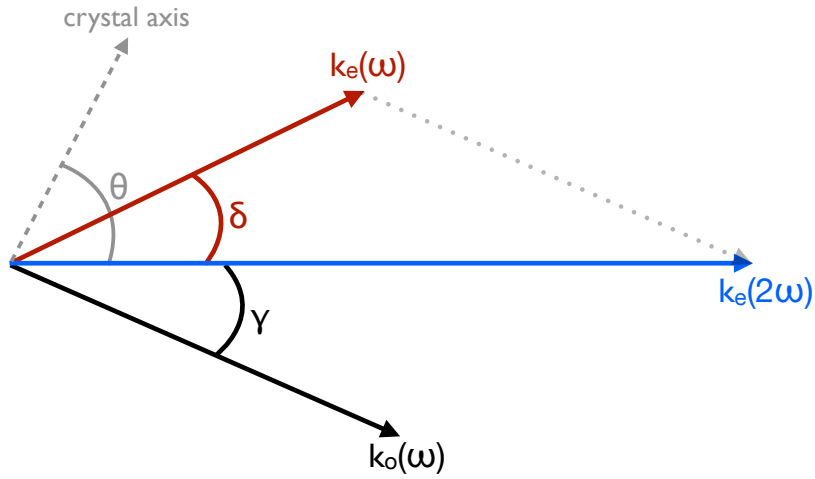

Figure 3.2: k-vectors

verse phasematching and the longitudinal phasematching independently. With $k=n \omega / c$, the transverse wavevector can be written in general as $\mathbf{k}_{\perp}=k \sin (\alpha)$ and the longitudinal wavevector as $\mathbf{k}_{\|}=k \cos (\alpha)$, where $\alpha$ denotes the angle between $\mathbf{k}_{e}(\omega)$ or $\mathbf{k}_{o}(\omega)$ and $\mathbf{k}_{e}(2 \omega)$. 


\section{Transverse phasematching}

In the case of transverse phasematching $\mathbf{k}_{e(2 \omega)}$ has no transversal components, thus is equal to zero. Equation 3.30 reduces to $\mathbf{k}_{o(\omega) \perp}+\mathbf{k}_{e(\omega) \perp}=0$, which can be written as

$$
\frac{n_{o(\omega)} \omega_{c}}{c} \sin (\gamma)=-\frac{n_{e(\omega)} \omega_{c}}{c} \sin (\delta) .
$$

Rearranging Eq. 3.31 gives as a solution for $\gamma$,

$$
\gamma=-\arcsin \left(\frac{n_{e(\omega)}}{n_{o(\omega)}} \sin (\delta)\right)
$$

\section{Longitudinal phasematching}

In this situation, $\mathbf{k}_{e(\omega) \|}+\mathbf{k}_{o(\omega) \|}=\mathbf{k}_{e(2 \omega) \|}$ can be written as

$$
\frac{n_{e(\omega)} \omega_{c}}{c} \cos (\delta)+\frac{n_{o(\omega)} \omega_{c}}{c} \cos (\gamma)=\frac{n_{e(2 \omega)} 2 \omega_{c}}{c} .
$$

Rearranging Eq. 3.33 and inserting Eq. 3.32 into Eq. 3.33 leads to

$$
2 n_{e(2 \omega)}-n_{e(\omega)} \cos (\delta)-n_{o(\omega)} \cos \left[\arcsin \left(\frac{n_{e(\omega)}}{n_{o(\omega)}} \sin (\delta)\right)\right]=0 .
$$

The extraordinary refractive index $n_{e}$ is dependent on the angle $\delta$ formed by the vectors $\mathbf{k}_{e(\omega)}$ and $\mathbf{k}_{e(2 \omega)}$ and on the crystal cut angle $\theta$. The numerical solutions for $\delta$ and $\gamma$ (Eq. 3.34 and Eq. 3.32) based on the ordinary and extraordinary index of $\beta$-Barium Borate are plotted for different crystal cut angles $\theta$ in Fig. 3.3.

\subsubsection{Angular dispersion}

If one wants the SF wave front normal to his wavevector $\mathbf{k}_{e(2 \omega)}$, the transverse components of the ordinary and the extraordinary wavevectors must fulfil the following condition, $\frac{d \mathbf{k}_{o(\omega) \perp}}{d \omega}=0$ and $\frac{d \mathbf{k}_{e(\omega) \perp}}{d \omega}=0$.

The frequency derivative of the transverse component of the ordinary wavevector $\mathbf{k}_{o(\omega)}$ is

$$
\frac{d \mathbf{k}_{o(\omega) \perp}}{d \omega}=\frac{\partial k_{o(\omega)}}{\partial \omega} \sin (\gamma)+k_{o\left(\omega_{c}\right)} \cos (\gamma) \frac{\partial \gamma}{\partial \omega} .
$$




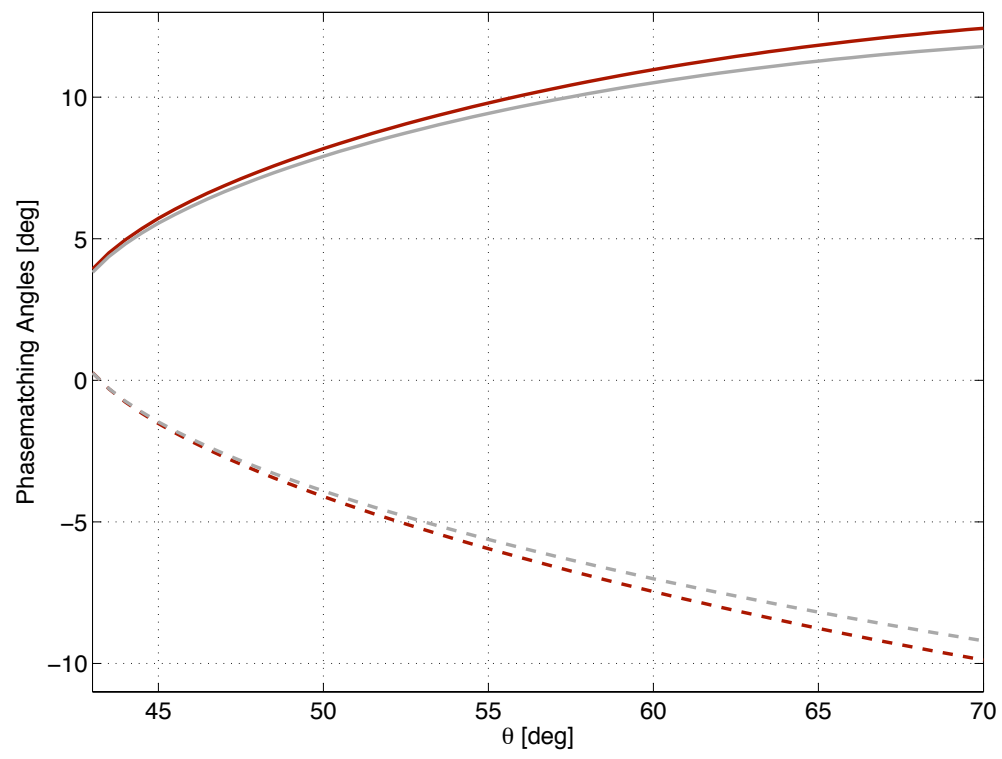

Figure 3.3: Positive (solid) and negative (dashed) solutions for the internal phasematching angles $\delta$ and $\gamma$ for the extraordinary (red) and the ordinary wave (grey) respectively as a function of the crystal cut angle $\theta$.

Rearranging Eq. 3.35 gives the angular dispersion for the ordinary wave:

$$
\frac{\partial \gamma}{\partial \omega}=-\frac{\partial k_{o(\omega)}}{\partial \omega} \frac{\tan (\gamma)}{k_{o\left(\omega_{c}\right)}}
$$

Note that $\frac{\partial k_{o(\omega)}}{\partial \omega}=\frac{1}{c} \frac{\partial\left(n_{o(\omega)} \omega\right)}{\partial \omega}$ is only a function of $\omega$ and does not depend on the noncollinear angle $\gamma$.

Analogous to the previous derivation the angular dispersion for the extraordinary phasematching angle $\delta$ has the form $\frac{\partial \delta}{\partial \omega}=-\frac{\partial k_{e(\omega)}}{\partial \omega} \frac{\tan (\delta)}{k_{e\left(\omega_{c}\right)}}$. But in this case there is a dependency on the noncollinear angle $\delta$, because

$$
\frac{\partial k_{e(\omega)}}{\partial \omega}=\frac{1}{c}\left(\frac{\partial n_{e(\omega)}}{\partial \omega} \omega_{c}+n_{e\left(\omega_{c}\right)}\right)
$$

and

$$
\frac{\partial n_{e(\omega)}}{\partial \omega}=-\frac{\partial n_{e\left(\omega_{c}, \delta\right)}}{\partial \delta} \frac{\partial \delta}{\partial \omega}+\frac{\partial n_{e(\omega, \delta)}}{\partial \omega} .
$$

After reorganising the terms, the angular dispersion of the extraordinary 
beam takes the form

$$
\frac{\partial \delta}{\partial \omega}=-\frac{\frac{\partial n_{e(\omega, \delta)}}{\partial \omega} \omega_{c}+n_{e\left(\omega_{c}, \delta\right)}}{\frac{\partial n_{e}\left(\omega_{c}, \delta\right)}{\partial \delta} \omega_{c}+n_{e}\left(\omega_{c}, \delta\right) \omega_{c} \cot \delta} .
$$

Figure 3.4 displays for various crystal cut angles $\theta$ the required angular dispersions of the extraordinary and the ordinary ray to get a sum-frequency pulse where the pulse front is normal to the propagation axis, i.e. untilted.

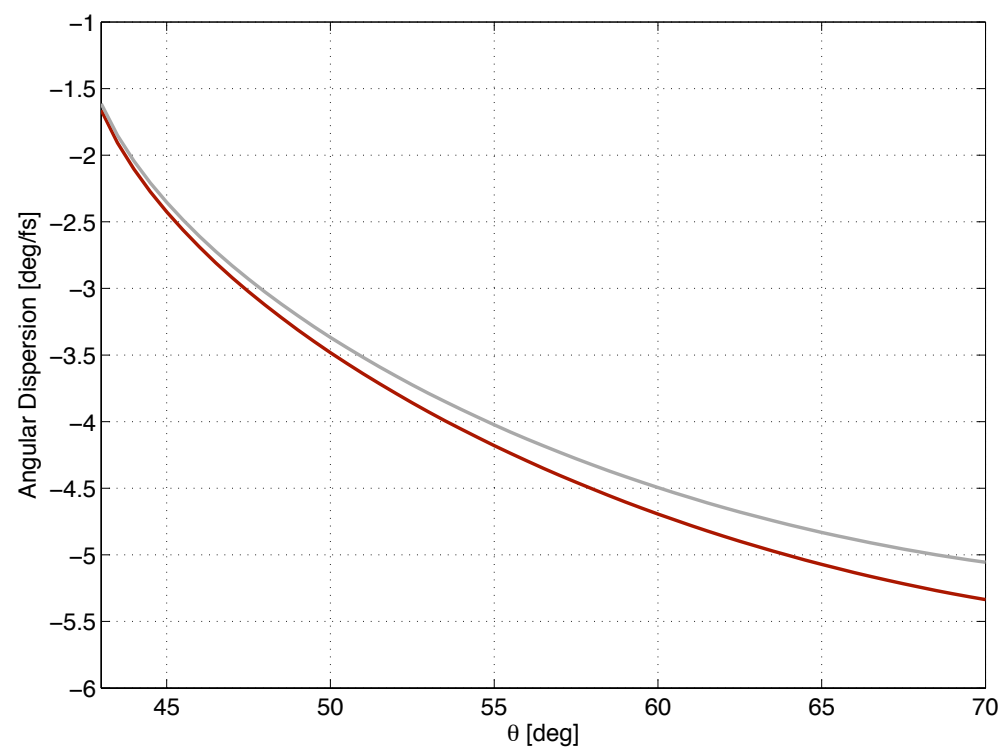

Figure 3.4: Angular dispersion of the ordinary (grey) and the extraordinary (red) wave as a function of the crystal cut angle $\theta$ resulting in a SF wave without pulse-front tilt.

\subsubsection{Group velocities}

In general, the group velocity $v_{g}$ is defined by the equation $v_{g}=\frac{\partial \omega}{\partial k} \cdot 150$ In order to find the appropriate group-velocity mismatch, which is essential for nonlinear pulse compression, one has to calculate the longitudinal derivative of the $k$-vectors with respect to the frequency, the inverse of the group velocity. The derivative of the ordinary and the extraordinary wavevectors has the form

$$
\frac{d \mathbf{k}_{o(\omega) \|}}{d \omega}=\frac{\partial k_{o(\omega)}}{\partial \omega} \cos (\gamma)-k_{o\left(\omega_{c}\right)} \sin (\gamma) \frac{\partial \gamma}{\partial \omega}
$$


and respectively

$$
\frac{d \mathbf{k}_{e(\omega) \|}}{d \omega}=\frac{\partial k_{e(\omega)}}{\partial \omega} \cos (\delta)-k_{e\left(\omega_{c}\right)} \sin (\delta) \frac{\partial \delta}{\partial \omega} .
$$

The group velocities depend on the noncollinear angles $\gamma$ and $\delta$ calculated in §3.2.1 and on the angular dispersion $\frac{\partial \gamma}{\partial \omega}$ and $\frac{\partial \delta}{\partial \omega}$ calculated in §3.2.2. The derivative of the sum-frequency generated wavevector has the simple form

$$
\frac{d \mathbf{k}_{e(2 \omega) \|}}{\partial \omega}=\frac{1}{c} \frac{\partial\left(n_{e(2 \omega)} \omega\right)}{\partial \omega} .
$$

Based on this equations the temporal group delays $\frac{d k}{d \omega}$ with respect to the SF group delay are shown in Fig. 3.5.

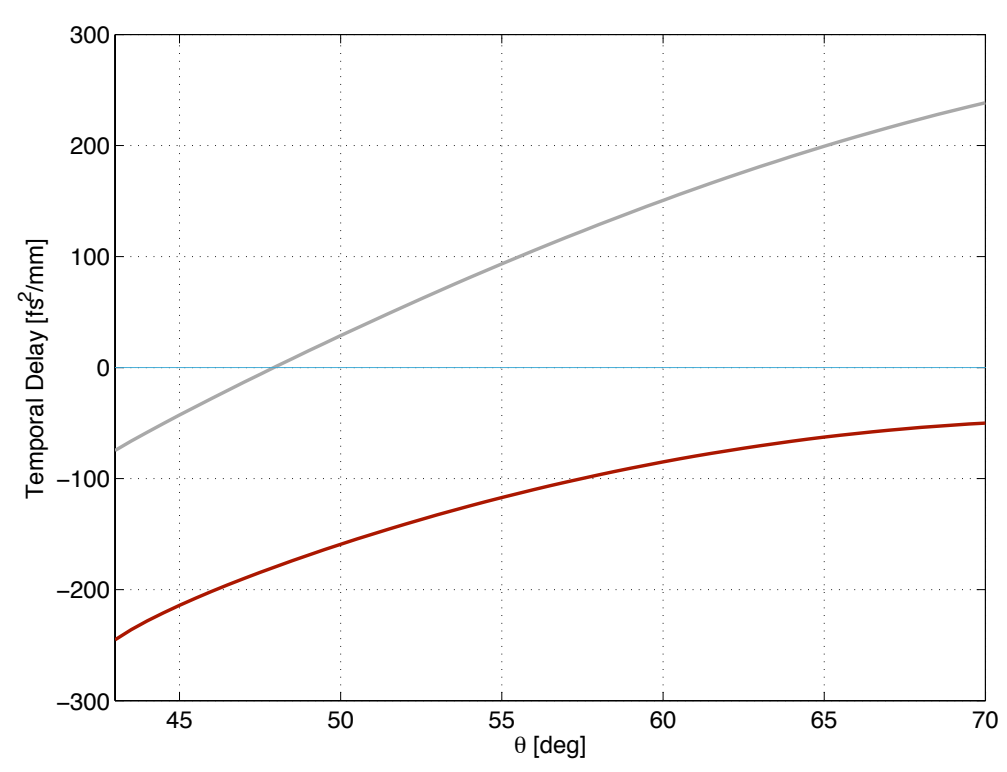

Figure 3.5: The temporal group delay differences of the ordinary (grey) and extraordinary (red) waves with the SF group delay (blue line) are plotted.

\subsubsection{Pulse-front tilt}

The pulse-front tilt (PFT) of an ultrashort laser pulse is generally considered to be a direct consequence of angular dispersion, i.e. the wavelength derivative of the angle of the spectral phase fronts. However, in the presence of spatial chirp the combination of the latter together with temporal 


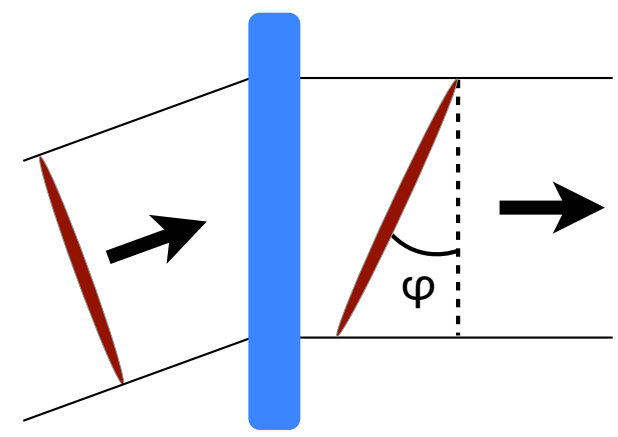

Figure 3.6: Pulse-front tilt of a pulse travelling through an angular dispersive element. On the input side the pulse and phase fronts are parallel. The pulse-front tilt is the angle $\varphi$ between the front of the electric field envelope maxima (red) relative to the phase front normal to the propagation direction (dashed line).

chirp can also cause a pulse-front tilt. For Gaussian beams and pulses, the following analytical relationship between angular dispersion and pulsefront tilt can be found:

$$
P F T=k_{0} \frac{d \varepsilon}{d \lambda}+\phi^{(2)} \nu
$$

where $k_{0}=2 \pi \lambda_{0}, d \varepsilon / d \lambda$ is the angular dispersion, $\nu$ is the spatial chirp, and $\phi^{(2)}$ is the temporal chirp due to group-delay dispersion. ${ }^{151,152}$ Here the temporal chirp is neglected and the pulse-front tilt can be treated linearly proportional to the angular dispersion independent of the source of angular dispersion. ${ }^{153}$ The pulse front tilt $\varphi$ can then be expressed as a function of the angular dispersion $d \epsilon / d \lambda$ as follows, ${ }^{154}$

$$
\tan \varphi=-\lambda_{0} \frac{d \epsilon}{d \lambda}
$$

where $\varphi$ is the tilt angle, the angle between the pulse front and the phase front (see Fig. 3.6) and $\lambda_{0}$ is the centre wavelength. The pulse front of an angular dispersed beam can be understood as the surface consisting of the points where the phase fronts of different wavelengths at different angles have the same phase, i.e. the points consisting the electric field envelope maxima. Such a case is illustrated schematically in Fig. 3.7 assuming that the beam propagates along the wavevector $\mathbf{k}(\lambda)$.

\section{Pre-compensation of the pulse-front tilt}

To find out the pulse-front tilt needed outside the crystal, the angular dispersion calculated in $\$ 3.2 .2$ was walked through the BBO-air interface taking 


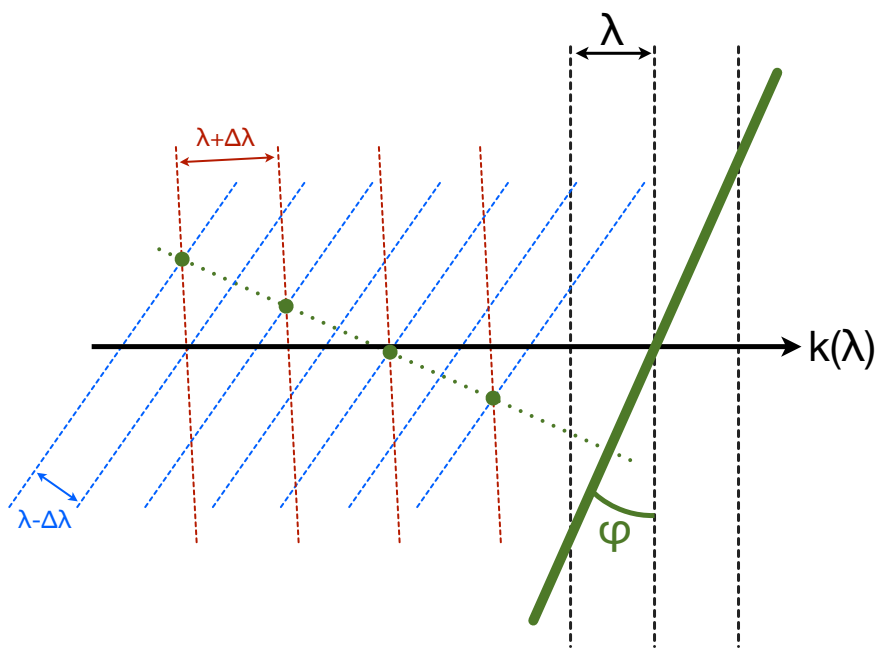

Figure 3.7: Two plane wave components at different wavelengths $(\lambda-$ $\Delta \lambda$ and $\lambda+\Delta \lambda$ ) of an angular dispersed pulse. The pulse front is the surface where the plane wave components with different wavelengths have the same phase.

into account Snell's law and then converted with Eq. 3.44. Figure 3.10(a) shows the PFT according to different BBO crystal cut angles $\theta$ at the outside of the crystal. There are different ways to induce the required PFT's. One could for example use a diffraction grating, a series of prisms or a prism combined with an imaging telescope. Here we treat the induced angular dispersion of a diffraction grating and the one induced by a prismtelescope. More details concerning the experimental implementations can be found in $§ 7.1$.

The familiar grating equation, presented here as Eq. 3.45, expresses the relationship between the groove density $G$, the diffracted order $m$, the diffracted wavelength $\lambda$, the angle of incidence $\alpha$, and the angle of diffraction $\beta$.

$$
G m \lambda=\sin \alpha+\sin \beta
$$

The angular dispersion $\frac{d \beta}{d \lambda}$ can be obtained by differentiating the grating equation, Eq. 3.45, assuming the incidence angle $\alpha$, the angle between the incident light and the normal to the grating, to be constant:

$$
\frac{d \beta}{d \lambda}=-m G /(\cos \beta) \text {. }
$$

To match the group-velocity mismatch conditions in a BBO type II crystal for ultrashort pulses centred at $800 \mathrm{~nm}$ (see Fig. 3.10(a)), a commercial 


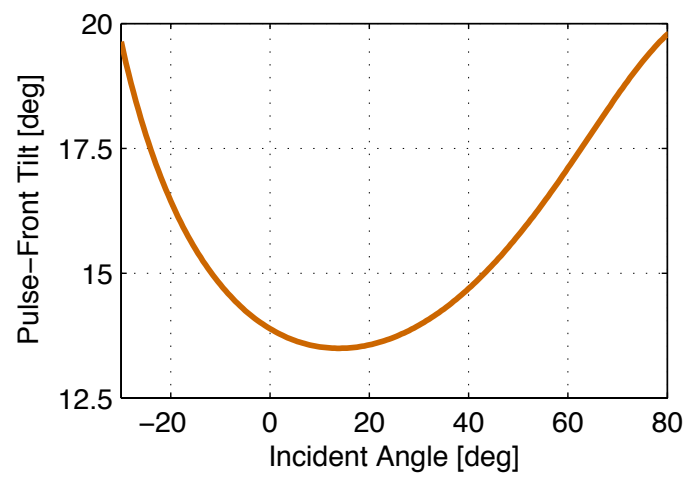

Figure 3.8: Pulse-front tilt induced by a diffraction grating with a groove density of 300 grooves $/ \mathrm{mm}$ as a function of the incident angle calculated for a pulse with a centre wavelength at $800 \mathrm{~nm}$.

available grating with a groove density of 300 grooves $/ \mathrm{mm}$ can be used. The induced pulse-front tilt of such a grating is shown in Fig. 3.8. Tuning the incident angle of the grating allows the pre-compensation of the pulsefront tilt for a particular range of crystal cut angles.

The angular dispersion induced by a dispersive prism can be deduced from Snell's law. According to this law, the incident and refracted angles of a prism with an apex angle A (see Fig. 3.9 for nomenclature conventions) can be calculated as follows:

$$
\begin{aligned}
\phi_{1}^{\prime} & =\arcsin \left(\sin \frac{\phi_{1}}{n}\right) \\
\phi_{2}^{\prime} & =A-\phi_{1}^{\prime} \\
\phi_{2} & =\arcsin \left(n \sin \phi_{2}^{\prime}\right) .
\end{aligned}
$$

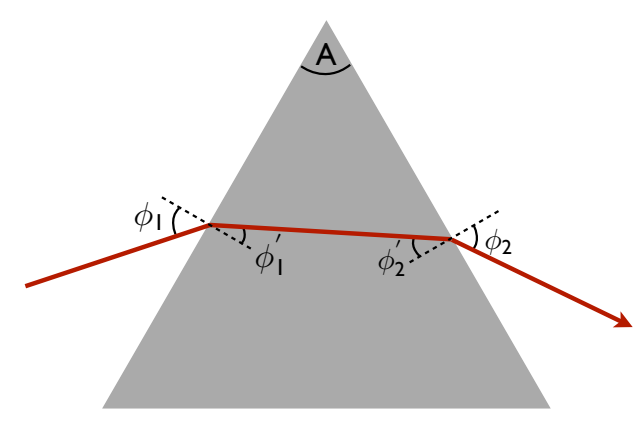

Figure 3.9: Nomenclature of a prism with an apex angle $A$, incident angle $\phi_{1}$ and refracted angles $\phi_{1}^{\prime}, \phi_{2}^{\prime}$ and $\phi_{2}$. 
At a given incident angle $\phi_{1}$ the induced angular dispersion of a prism is then given by

$$
\frac{d \epsilon}{d \lambda}=-\frac{\sin A}{\cos \phi_{1}^{\prime} \cos \phi_{2}} \frac{d n}{d \lambda} .
$$

Prisms suitable for the near-infrared are usually made out of borosilicate crown or flint glass, for instance BK7 and F2. The resulting angular dispersion induced by such prisms is too small to compensate the required PFTs in BBO. A solution is to put a set of lenses after the prism, i.e. to built a prism-telescope, and consequently magnify the pulse-front tilt. As the tilted pump pulses pass through a telescope with lenses of focal lengths $f_{1}$ and $f_{2}$, the pulse front is imaged with a longitudinal magnification factor $f_{1} / f_{2}$ so that the external pulse-front tilt at the crystal surface is determined by

$$
P F T_{\text {in }}=\frac{f_{1}}{f_{2}} P F T_{\text {out }} .
$$

With a prism-telescope consisting of an equilateral dispersive prism made out of $\mathrm{F} 2$ and an, $f_{1} / f_{2}=6.67$, imaging telescope, one can achieve the pulse-front tilt's plotted in Fig. 3.10(b).

(a)

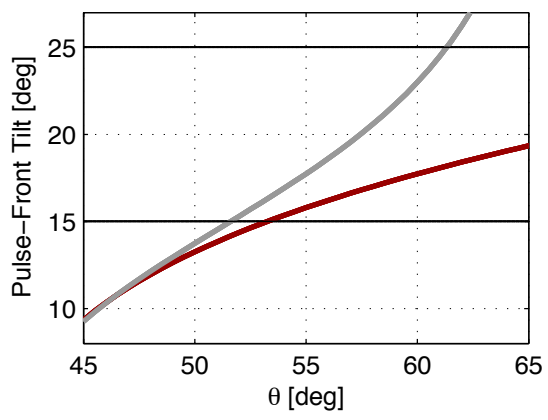

(b)

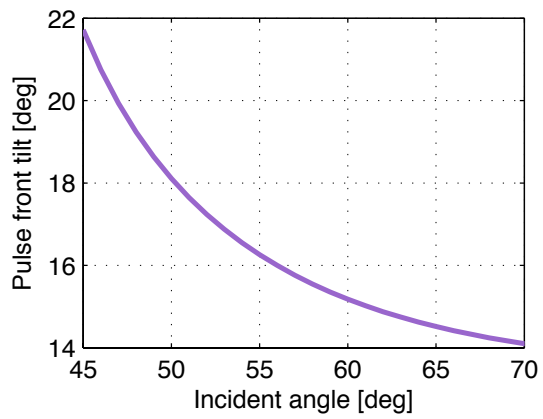

Figure 3.10: (a) Pulse-front tilts required at the outside of the BBO crystal to match the phasematching conditions calculated at the inside of the latter.(b) induced PFT of a prism-telescope for a certain range of incident angles $\phi_{1}$. The prism-telescope consists of an equilateral prism made out of F2 and an imaging telescope with $f_{1}=500 \mathrm{~mm}$ and $f_{2}=75 \mathrm{~mm}$. 


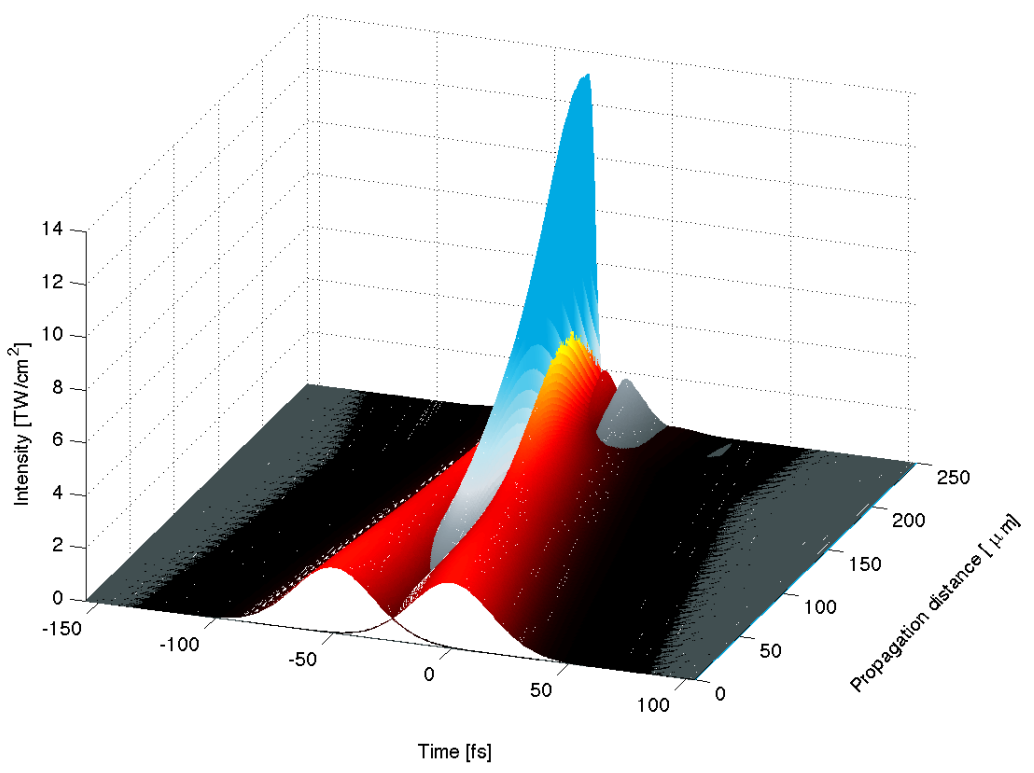

The highly nonlinear evolution of an atom in an intense laser field prevents its analytical calculation. Instead numerics are required to investigate the system's dynamics. This chapter contains a description of the code simulating the sum frequency generation process inside a nonlinear medium with it's basic approximations. The main numerical results are then presented to obtain a deeper understanding of the physics involved in this three wave interaction. For that a parameter space representing the degrees of freedom in the experiment is investigated, e.g., fundamental pulse durations and intensities, initial pre-delay, and crystal cut angle, thus clarifying their dependence on the self compression of the sum-frequency generated laser pulse. 


\section{Chapter 4}

\section{Numerical simulations}

\subsection{Simulation properties and parameters}

A MATLAB code was developed able to simulate accurately a noncollinear SFG process and the nonlinear interaction driven by intense laser pulses, taking into account both group velocity mismatch and dispersion spreading of the pulses in a type II BBO crystal of $250 \mu \mathrm{m}$ length. The numerical simulation is carried out following the physical model in Chap. 3, i.e. calculating the numerical solutions of the set of nonlinear coupled equations (Eq. 3.26) using a first order differential equation solver, ode $45^{1}$. The propagation of the three interacting pulses is carried out in one dimension (1D).

The two fundamental NIR laser pulses have their electric-field vectors aligned in the ordinary and extraordinary polarization state, thus traveling with the ordinary and extraordinary group velocities inside the negative uniaxial crystal. The ordinary group velocity does not depend on any angle, whereas the extraordinary group velocity depends on the incident noncollinear angle and on the crystal cut angle. The SFG wave will then emerge with the electric-field vector aligned along the extraordinary polarization state. The one dimensional pulse propagation code uses the projections of the ordinary and extraordinary $k$-vectors onto the propagation axis of the SF beam, i.e. the simulation axis. The wavevectors contain the angular dispersion and the noncollinear angles deduced in Chap. 3 and ensure the optimal phasematching conditions and the appropriate groupvelocity mismatch with the energy fronts of all pulses parallel in the crystal.

For the ordinary and extraordinary refractive indices $n_{o}$ and respectively

\footnotetext{
${ }^{1}$ MATLAB's standard solver for ordinary differential equations (ODEs). This function implements a Runge-Kutta method with a variable time step for efficient computation.
} 
$n_{e}$, the full Sellmeier equation for $\mathrm{BBO}^{155}$

$$
\begin{aligned}
& n_{o}^{2}=2.7359+\frac{0.01878}{\lambda^{2}-0.01822}-0.01354 \lambda^{2} \\
& n_{e}^{2}=2.3753+\frac{0.01224}{\lambda^{2}-0.01667}-0.01516 \lambda^{2}
\end{aligned}
$$

were included, where $\lambda$ is given in micrometer. Because the frequency dependence of the $k$-vectors is taken into account, all group-velocity dispersion effects as they affect the pulse propagation and the pulse conversion process are included in the simulation.

Determining the effective second-order nonlinear coefficients, $d_{e f f}$, of BBO is rather complex. In early studies this crystal was erroneously assigned to a point group symmetry 3 and consequently the following equations $^{156}$

$$
\begin{aligned}
& d_{\text {eff }}^{\text {ooe }}=d_{15} \sin \theta+\cos \theta\left(d_{11} \cos 3 \phi-d_{22} \sin 3 \phi\right) \\
& d_{\text {eff }}^{\text {eoe }}=d_{\text {eff }}^{\text {oee }}=\left(d_{11} \sin 3 \phi+d_{22} \cos 3 \phi\right) \cos ^{2} \theta
\end{aligned}
$$

were used for calculating the effective nonlinearities, where $\phi$ and $\theta$ are the polar and azimuthal angles in a polar coordinate system related to the crystallographic axes of the crystal. In further investigations ${ }^{157}$ it was found that $\beta$-Barium Borate actually belongs to the symmetry group $3 \mathrm{~m}$ for which the expression for $d_{\text {eff }}$ corresponds to the above-given equations Eq. 4.3 and Eq. 4.4 but with $d_{11}=0$, that is

$$
\begin{aligned}
& d_{\text {eff }}^{\text {ooe }}=d_{15} \sin \theta-d_{22} \cos \theta \sin 3 \phi \\
& d_{\text {eff }}^{\text {eoe }}=d_{\text {eff }}^{\text {oee }}=d_{22} \cos ^{2} \theta \cos 3 \phi .
\end{aligned}
$$

Hence it follows that a maximum value of $d_{e f f}$ is achieved at $\phi=90^{\circ}$ in the case of an ooe type interaction and at $\phi=0^{\circ}$ in the case of eoe and oee interactions. ${ }^{158}$ In this simulation an eoe interaction is studied, thus Eq. 4.6 reduces to:

$$
d_{e f f}^{e o e}=d_{22} \cos ^{2} \theta .
$$

with $d_{22}=2.3 \cdot 10^{12}$ taken from reference ${ }^{159}$ and $\theta$ being the crystal cut angle. In the coupled wave equation, Eq. 3.26, the second order nonlinear susceptibility $\chi^{(2)}$ is used which differs from the effective nonlinearity by a factor 2 ,

$$
d_{e f f}=\frac{\chi^{(2)}}{2}
$$


The differential equations are evaluated over a propagation distance of $250 \mu \mathrm{m}$ using an adaptive step size and keeping the local error tolerance below $10^{-11}$. Note that for the following simulations, the resulting pulse properties are taken after the full propagation distance.

Another sensible option would have been to study the pulse properties based on the maximum power of the sum-frequency signal or the maximum conversion efficiency, which do not necessarily appear at the end of the crystal. The peak power and the conversion efficiency are usually good indicators for short pulse durations. As the interaction length strongly depends on the input pulse durations, e.g., for very short pulses ( $\sim 10 \mathrm{fs}) 50 \mu \mathrm{m}$ would be an adequate interaction length, the shortest pulse can arise before the end of the crystal. Subsequent reconversion together with the induced material dispersion over the remaining path can result in temporal pulse broadening. For pulses exceeding 20 fs the pulse broadening caused by the group delay dispersion of $250 \mu \mathrm{m}$ BBO can be neglected. Assuming Gaussian pulses, the pulse duration, $\tau_{\text {out }}$, after propagation through a dispersive medium ${ }^{160}$ can be estimated with the following equation:

$$
\tau_{\text {out }}=\frac{\sqrt{\tau_{\text {in }}^{4}+16 \ln (2)^{2} G D D^{2}}}{\tau_{\text {in }}},
$$

where GDD is the sum of the group delay dispersion of the material and $\tau_{i n}$ is the input pulse duration. Applying Eq. 4.9 to the three waves involved in the sum-frequency process, see Fig. 4.1, one can see that the pulse broadening after $250 \mu \mathrm{m}$ BBO is less than $2 \%$ for pulses longer than 20 fs. Given that the typical pulses delivered by a Ti:sapphire laser system exceed $25 \mathrm{fs}$, that the unwanted reconversion can be partially compensated by choosing a suitable pre-delay, and in order to meet the experimental conditions, this option was discarded.

The code uses Gaussian-shaped input-fields. The parameters of interest related to the nonlinear pulse compression under study are listed below:

- Pulse duration: The pulse width in the time domain is defined in the intensity picture, $\tau_{\text {Intensity }}=\frac{F W H M}{\sqrt{2 \ln (2)}}$. Whereas for the electrical field it would be $\tau_{\text {el.Field }}=\frac{F W H M}{2 \sqrt{\ln (2)}}$. The initial ordinary and extraordinary pump pulse durations are always chosen to be equal.

- Intensity: The intensity is defined as the maximum amplitude of the electric field. 


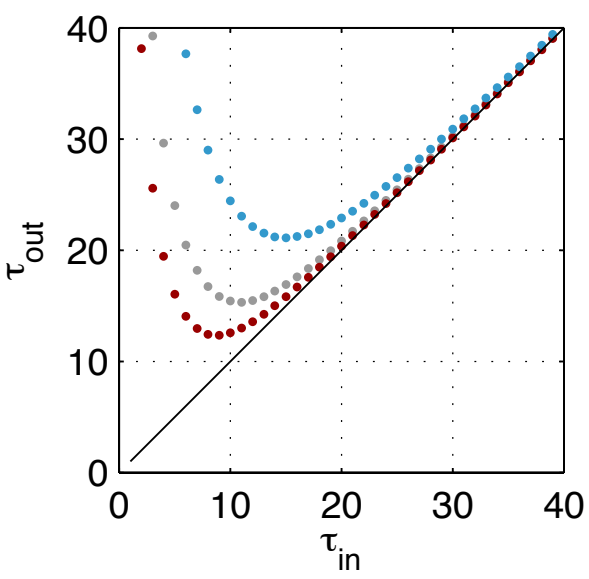

Figure 4.1: Near-infrared ordinary and extraordinary polarized (grey and red) and sum-frequency (blue) pulse durations after propagation through $250 \mu \mathrm{m}$ of BBO.

- Wavelength: The centre wavelength is kept at $785 \mathrm{~nm}$ for all simulations.

- Phase: 2nd, 3rd, and 4th order dispersion as well as an intensitydependent phase shift can be applied to the incident pump pulses.

- Pre-delay: The pre-delay is the time difference between the ordinary and the extraordinary pulse and is chosen to be 1,25 times the input FWHM of the fundamentals.

- The compression factor is defined as the ratio between the FWHM of the sum-frequency generated pulse and the one of the fundamental frequency pulses.

Besides Gaussian pulses, there exists the possibility of implementing reconstructed Ti:sapphire laser pulses measured with a home built SHGFROG or an acousto-optic pulse shaper (PHAZZLER). ${ }^{161}$

\subsection{Numerical results of the simulation}

In this section a sensible parameter space is scanned in order to set the limiting conditions for efficient pulse compression. First the necessary intensities leading to strong energy exchange between the interacting waves are evaluated. Then the dependence of the crystal cut angle is investigated 
followed by the optimum pre-delay. Another section treats the effects related to the dispersion and to the nonlinear phase shift. Having found a promising set of parameters the pulse evolution along the crystal is shown. Finally the conditions under which shortening of the incident NIR pulses occurs are studied.

\subsubsection{Strong energy exchange}

To estimate which intensities are needed to ensure effective energy exchange, thus depletion of the pump pulses, thus shortening of the overlap area and thus pulse compression, input intensities, $I_{i n}=I_{o}+I_{e}$, ranging from $0.1 \mathrm{TW} / \mathrm{cm}^{2}$ to $10 \mathrm{TW} / \mathrm{cm}^{2}$ are simulated. $I_{o}$ and $I_{e}$ are the peak intensities of the ordinary respectively the extraordinary pulse. For this simulation the intensity ratio is set to $I_{e} / I_{o}=1$, the input full width half maximum (FWHM) of both pump pulses is $40 \mathrm{fs}$, and the crystal angle $\theta$ is kept at $55^{\circ}$.

Figure 4.2 shows the FWHM of the sum-frequency pulses at the end of the crystal for different pump intensities. A clear trend towards shorter pulses with increasing input intensities is apparent. For a first orientation the SF FWHM pulse widths map the intensity range where one can expect nonlinear pulse compression quite well.

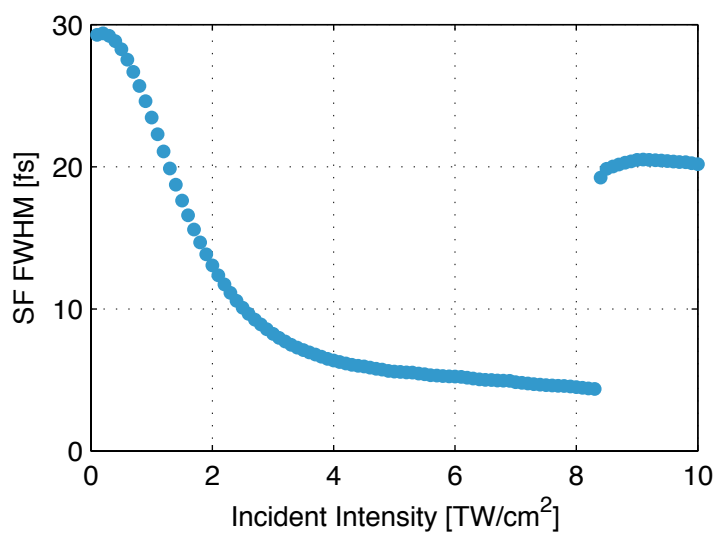

Figure 4.2: Resulting SFG pulse width in dependence of the input intensity $I_{i n}$. Starting with $40 \mathrm{fs}$ short pulses, at least $0.5 \mathrm{TW} / \mathrm{cm}^{2}$ are needed to see the desired effect.

However, for a deeper understanding of this nonlinear process, one has to look at the structure of these pulses, e.g., why are the SF pulse durations increasing again after a certain incident intensity. Figure 4.3 shows 
the electric field intensities in the time and frequency domain corresponding to the calculated points in Fig. 4.2. At incident peak power intensities

(a)

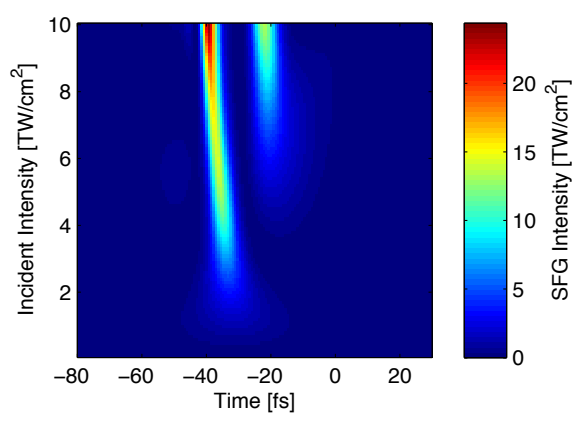

(b)

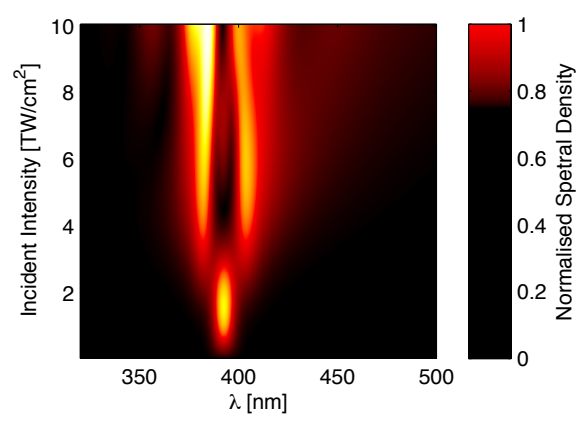

Figure 4.3: sum-frequency (SF) generated pulse shapes after $250 \mu \mathrm{m}$ of propagation in BBO $\left(\theta=55^{\circ}\right)$ for incident intensities $\left(I_{i n}=I_{e}+I_{o}\right)$ ranging from $0.1 \mathrm{TW} / \mathrm{cm}^{2}$ to $10 \mathrm{TW} / \mathrm{cm}^{2}$. The input pulse duration is 40 fs FWHM.(a) Temporal Intensity distribution. (b) SFG spectra.

lower than $0.5 \mathrm{TW} / \mathrm{cm}^{2}$, low conversion efficiencies accompanied by negligible depletion of the NIR pulses lead to pulse durations around $30 \mathrm{fs}$. This is expected for Gaussian shaped input pulses as the SHG shortening factor in this regime is $\sqrt{2}^{160}$ (40 fs $/ \sqrt{2} \approx 28 \mathrm{fs}$ ). For intensities exceeding $2 \mathrm{TW} / \mathrm{cm}^{2}$ spectral broadening is observed which is the result of the sumfrequency pulse amplitude modulation. Around $4 \mathrm{TW} / \mathrm{cm}^{2}$ a characteristic feature of nonlinearly compressed pulses appears; a specific doublepeaked spectrum. The SF pulse durations are decreasing until $8 \mathrm{TW} / \mathrm{cm}^{2}$ where 5 fs short pulses can be generated. At this intensities a side lobe emerges in the time domain. This is due to the reconversion from the SF generated wave to the pump pulses and back to the sum-frequency wave. More details about this process can be found in $\S 4.2 .5$. For pump intensities greater than $8 \mathrm{TW} / \mathrm{cm}^{2}$, the side lobe becomes more dominant and exceeds half of the peak intensity; a double pulse is formed which explains the longer SF pulse width.

\subsubsection{Dependence of the crystal cut angle}

The crystal cut angle $\theta$ is a critical parameter. On one hand the groupvelocity mismatch depends strongly on $\theta$ (see Fig. 3.5), on the other hand the second order nonlinear coefficient $\chi^{2}$ is proportional to the crystal angle (see Fig. 4.4(b)). Here the influence of the crystal cut angle in the range of 
$45^{\circ}$ to $70^{\circ}$ is investigated. The aim of this simulation is to find the optimum balance between the appropriate group-velocity mismatch combined with the highest possible energy exchange between the waves. Here the input intensity, $I_{i n}=I_{o}+I_{e}$, is kept fixed at $5 \mathrm{TW} / \mathrm{cm}^{2}$, the intensity ratio $I_{e} / I_{o}=$ 1 , and the input FWHM of both pump pulses is $40 \mathrm{fs}$.

(a)

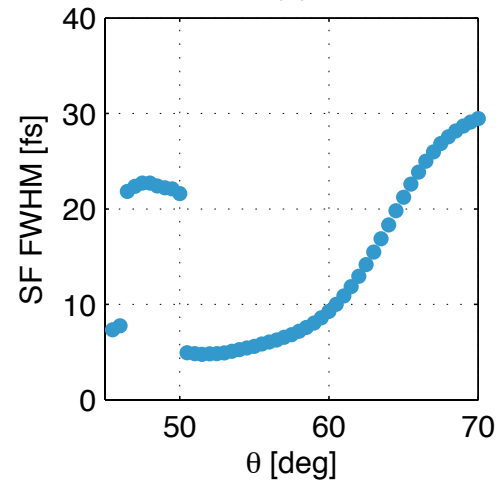

(b)

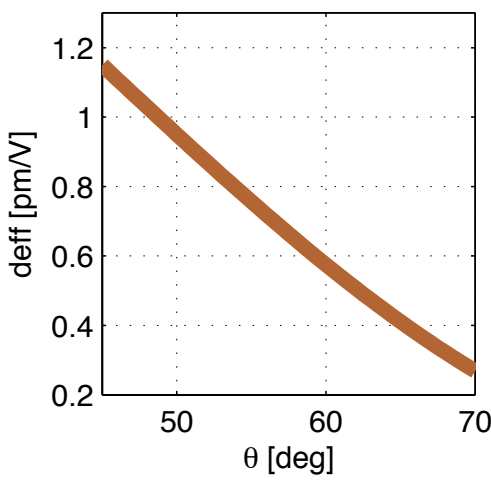

Figure 4.4: (a) Resulting SFG pulse durations (FWHM) in dependence of the crystal cut angle $\theta$. (b) Second order nonlinear susceptibility calculated following Eq. 4.7.

For crystal cut angles smaller than $50^{\circ}$ the group-velocity mismatch condition, $v_{1}<v_{S F}<v_{2}$, is not fulfilled resulting in pulse distortions and SF pulse durations higher than 22 fs. The range between $50^{\circ}$ and $60^{\circ}$ seems to be a promising regime for nonlinear pulse compression. Because the energy transfer depends strongly on $\chi^{2}$ and the latter is decreasing with increasing $\theta$, choosing $\theta>60^{\circ}$ is rather unfavourable for pulse compression: Despite the fact that the GVM condition is fulfilled, higher intensities are required.

Figure 4.5 shows the pulse shapes in the time and in the frequency domain according to the crystal cut angle. The overall trend is very similar to the one discussed in the previous section. Instead of going to higher intensities, the bigger energy transfer triggers the nonlinear pulse compression. When the crystal cut angle is increased, spectral broadening together with pulse compression is observed. The group-velocity mismatch doesn't affect the result as much as the second order nonlinear susceptibility. It is sufficient that the SF group velocity lies between the ordinary and the extraordinary fundamental group velocities. The group velocity differences $\left|v_{1}-v_{S F}\right|$ and $\left|v_{2}-v_{S F}\right|$ certainly don't need to be symmetric around the SFG signal. With increasing $\theta$ the pulse is shifted in time, i.e. occurs after a 
(a)

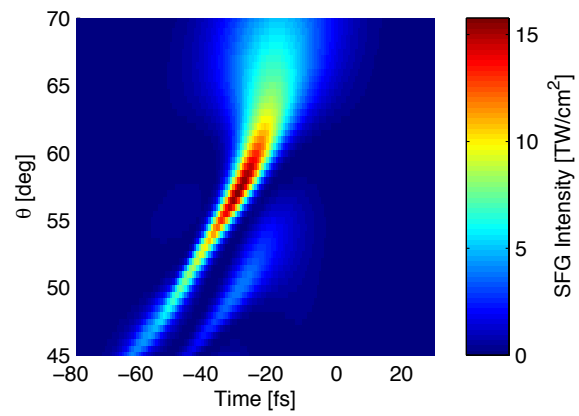

(b)

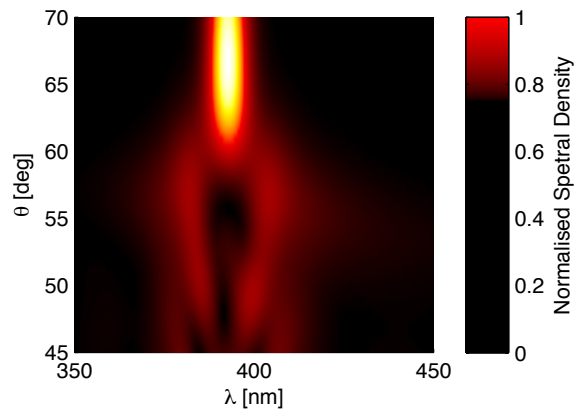

Figure 4.5: SF generated pulse shapes after $250 \mu \mathrm{m}$ of propagation in BBO for incident intensities $I_{i n}=I_{e}+I_{o}=5 \mathrm{TW} / \mathrm{cm}^{2}$. The input pulse duration is 40 fs FWHM.(a) Temporal Intensity distribution. (b) SF spectra.

longer propagation distance. This is because the group velocities vary with $\theta$. Depending on the chosen incident intensity, one has to carefully look at the intensity- $\chi^{2}$ product, i.e. the crystal cut angle $\theta$. A higher intensity$\chi^{2}$ product leads to a faster energy exchange and hence to a shorter SF pulse. As long as the group-velocity mismatch condition is not violated, a smaller crystal cut angle $\theta$, entailing asymmetric group velocity differences with respect to the SF pulse, is advantageous for lower pump intensities.

\subsubsection{Pre-delay}

In this section the role of the pre-delay is examined. The pre-delay is defined as the time difference between the ordinary and the extraordinary NIR pulses at the start of the simulation. A positive pre-delay means that the ordinary pulse starts earlier in time with respect to the extraordinary pulse. As the ordinary ray has a slower group velocity than the extraordinary ray in BBO, no complete temporal overlap of the fundamentals can occur at negative pre-delays, i.e the extraordinary pulse runs away. In the experiment a negative pre-delay corresponds to an increase of the ordinary beam path and respectively a positive pre-delay corresponds to a decrease of the ordinary beam path.

The optimum pre-delay strongly depends on the input pulse durations and on the incident pulse intensities. Quite evidently, shorter input pulse durations require shorter pre-delays. At higher intensities shorter predelays are advantageous as the energy conversion from the near-infrared 
(a)

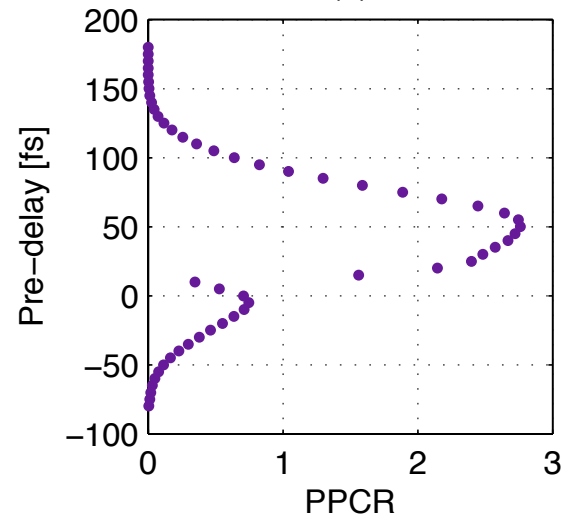

(b)

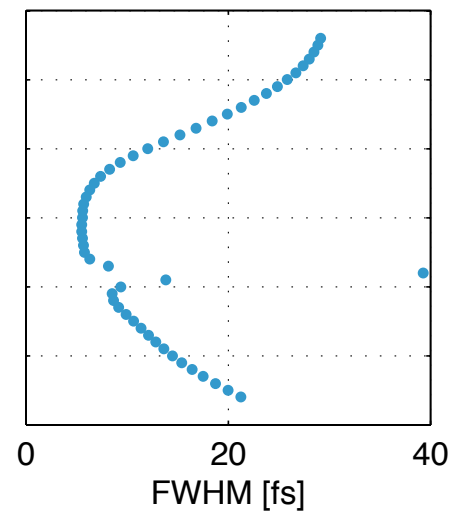

Figure 4.6: (a) Peak power conversion rate (PPCR) and (b) full width half maximum of the SF generated pulse as a function of the pre-delay. The $y$-axis is the same for the two graphs.

waves to the sum-frequency generated wave takes place much faster. Figure 4.6 shows a typical evolution of the peak power conversion rate together with the FWHM SF pulse duration as a function of the pre-delay. The intensity was chosen to be high enough to ensure depletion of the NIR pulses. At negative pre-delays the extraordinary wave runs away and the fundamental beams never overlap in time. At slightly negative pre-delays the Gaussian tails of the fundamentals overlap sufficiently at the beginning of the crystal to generate some SF signal. If nearly no pre-delay is applied, the incident pulses are fully overlapped at the beginning making the overlap region highest which explains the longer SF pulse durations. With increasing pre-delays, depletion of the fundamentals sets in, the temporal overlap region is reduced and pulse shortening can be observed. At longer pre-delays the distance for the extraordinary pulse to catch up the ordinary pulse becomes too long. As a consequence, the peak power conversion rate decreases.

The generated sum-frequency spectra corresponding to the different pre-delays can give interesting insights into the process. In Fig. 4.7 the resulting SF spectra are plotted for three different incident intensities while the input pulse duration was kept at $40 \mathrm{fs}$. At lower intensities the graph nearly represents a SHG-FROG trace with the difference that the spectra span over a larger time range compared to a proper FROG trace. This is due to the long crystal length of $250 \mu \mathrm{m}$. As the incident intensities increase, one can observe spectral broadening and the formation of a spec- 
(a)

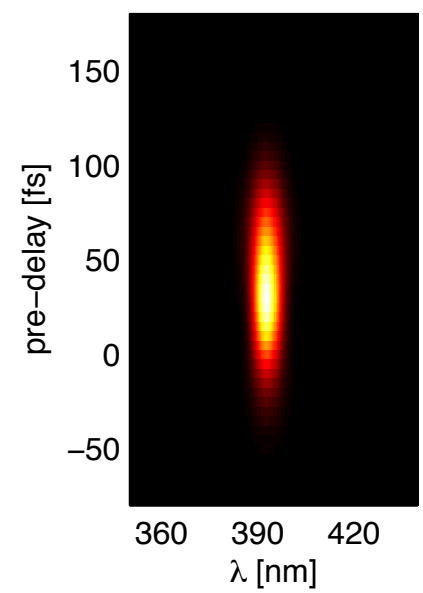

(b)

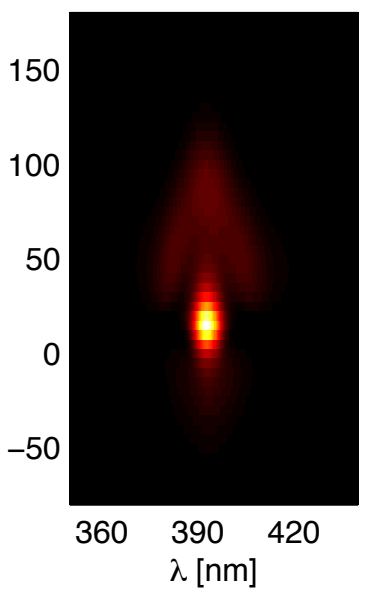

(c)

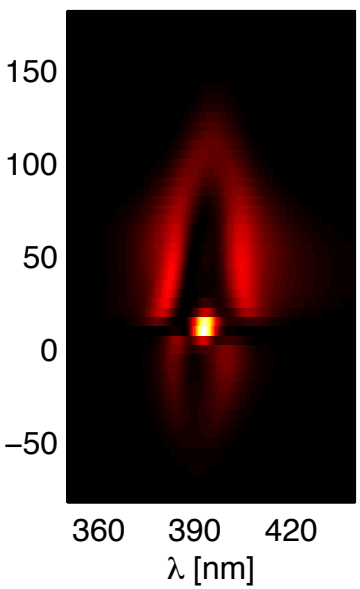

Figure 4.7: Spectra of the sum-frequency generated pulses as a function of the pre-delay for incident peak pulse Intensities at (a) $1 \mathrm{TW} / \mathrm{cm}^{2}$, (b) $3 \mathrm{TW} / \mathrm{cm}^{2}$, and (c) $5 \mathrm{TW} / \mathrm{cm}^{2}$. The $\mathrm{y}$-axis is the same for all graphs.

tral hole later on. The higher the input intensities the faster the energy conversion to the SF takes place and the earlier an ultrashort pulse with a high peak power emerges.

\subsubsection{Dispersion and nonlinear phase}

Chirping the pulses mainly results in longer input pulse durations. The bigger the chirp, regardless of the sign, the longer the SF pulse full width half maximum (see Fig. 4.8(a)). This can be explained by the fact that by chirping the incident pulse, the input pulse duration is stretched. At the same time the peak intensity is reduced. As a result less energy is exchanged between the three waves, the fundamentals do not get depleted and the temporal overlap region remains in the range of the fundamentals. Thus longer near-infrared pump pulses generate longer SF pulses when the pump intensity is kept constant.

In order to compress ultrashort pulses, very high optical intensities are needed. As a consequence intensity-dependent effects such as self-phase modulation may arise. The pulsed change of optical intensity can cause a fast variation of the phase encountered by the pulsed beam itself. And according to the principle of Fourier transformation, a fast phase modulation will lead to a broadening of the frequency spectrum. By implementing an intensity-dependent nonlinear phase shift, which is applied on the NIR 
(a)

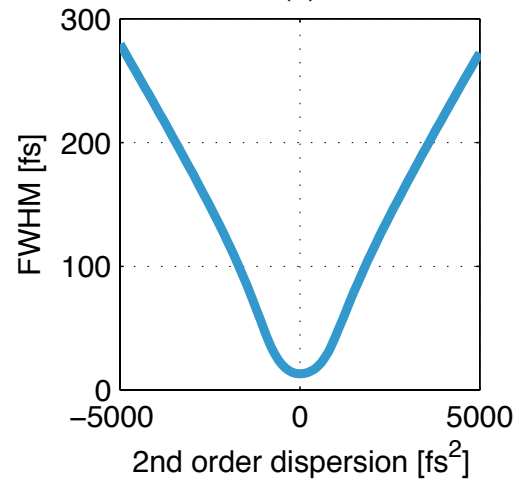

(b)

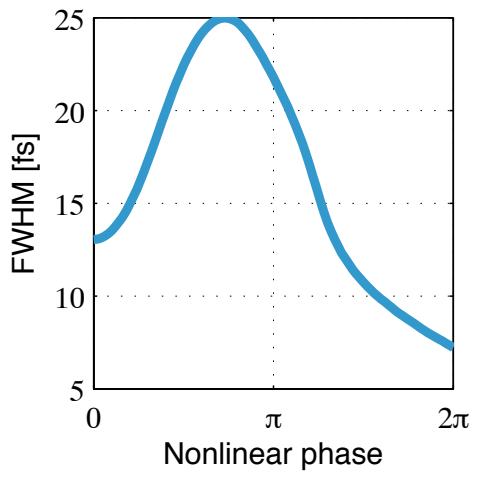

Figure 4.8: SF FWHM after $250 \mu \mathrm{m}$ of propagation in BBO as function of (a) the $2^{\text {nd }}$ order dispersion and (b) the nonlinear phase shift.

pump pulses in the time domain, these effects can be studied. Figure 4.9 shows the influence of the nonlinear phase shift on an unchirped Gaussian pulse for five different cases. The SPM-induced spectral broadening is accompanied by a multipeak structure. The number of peaks depends on the maximum nonlinear phase shift and increases linearly with it. The oscillatory structure is a result of the interference of waves of the same frequency but with different phases. ${ }^{162}$
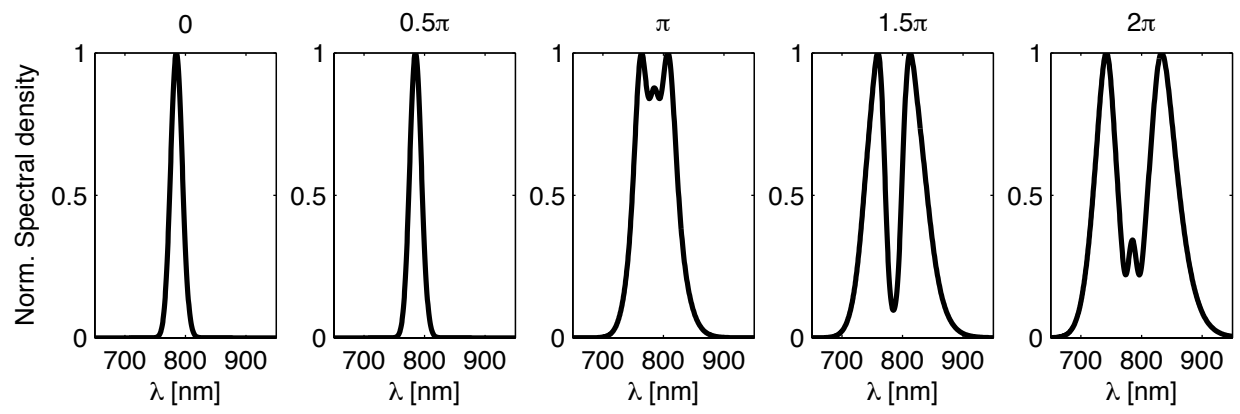

Figure 4.9: Influence of the nonlinear phase shift on an unchirped Gaussian pulse. Spectra are labeled by the maximum nonlinear phase shift.

Figure 4.8(b) shows the resulting SF pulse width after an interaction length of $250 \mu \mathrm{m}$ for nonlinear phase shifts ranging from 0 to $2 \pi$. The incident intensity was set to $2 \mathrm{TW} / \mathrm{cm}^{2}$. With increasing nonlinear phase shifts the sum-frequency generated pulses get longer until the nonlinear phase shift reaches $0.8 \pi$. For nonlinear phase shifts bigger than $0.8 \pi$, the sum-frequency pulse durations are decreasing and for phase shifts bigger 
than $1.3 \pi$ even an enhancement of the compression is visible.

\subsubsection{Pulse Evolution in the crystal}

In this section the evolution of the sum-frequency generation process will be examined in the time domain. In order to give a full picture of the SFG interaction being presented, it is vital to display the SF pulses as well as the near-infrared pump pulses. The intensities of the three waves involved in the sum-frequency generation process were written into files after each micrometer.

(a)

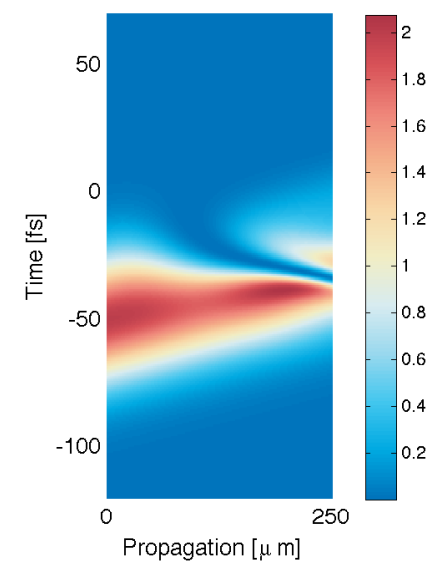

(b)

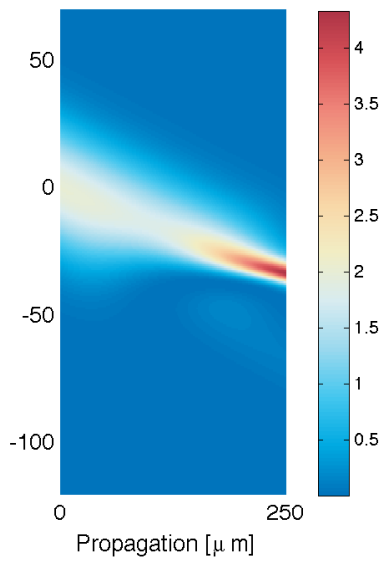

(c)

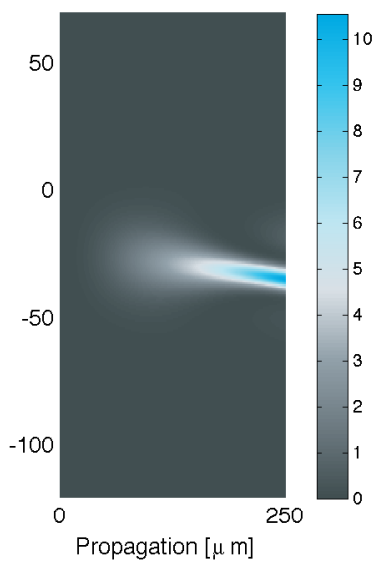

Figure 4.10: Temporal pulse evolution of the (a) ordinary, (b) extraordinary polarized pump, and (c) SF pulse in a $250 \mu \mathrm{m}$ long BBO crystal. All the intensity scales are in $\mathrm{TW} / \mathrm{cm}^{2}$.

Figure 4.10 shows the numerical results of a simulation starting with 40 fs long fundamental frequency pulses, where the ordinary polarized pulse is delayed by 50 fs with respect to the extraordinary polarized one. The crystal cut angle is chosen to be $55^{\circ}$ and the total incident intensity is $4 \mathrm{TW} / \mathrm{cm}^{2}$. The pulse intensities are plotted in the time domain as a function of the propagation distance within the BBO crystal. Figure 4.11 represents the corresponding fluences to outline the energy transfer between the NIR ordinary, extraordinary, and the sum-frequency wave.

The starting point of the simulation is equivalent to a propagation distance of $0 \mu \mathrm{m}$. The $o$ - and the e-polarized pump pulses are delayed by $50 \mathrm{fs}$, just the pulse tails are overlapping, and all the energy is in the fundamentals. Until $50 \mu \mathrm{m}$ the pump pulses approach each other, the overlap region augments constantly and the energy transfer to the sum-frequency 


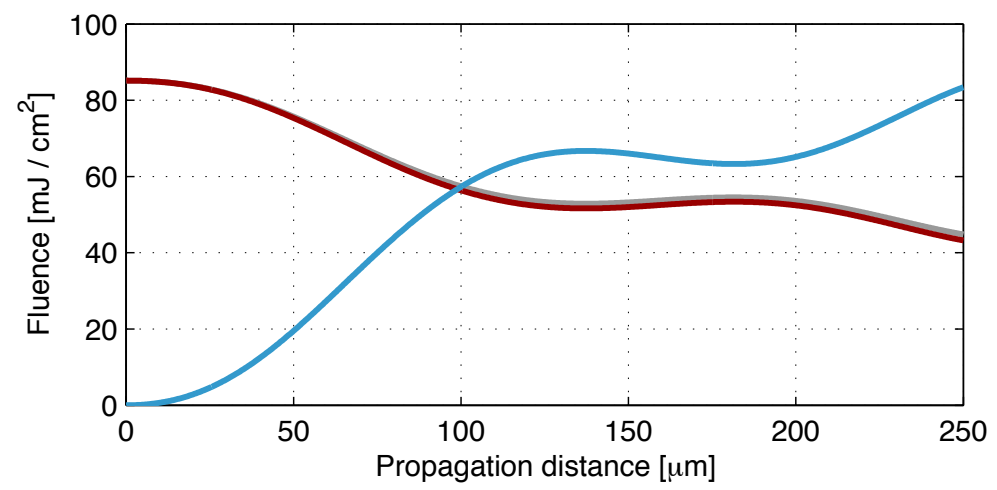

Figure 4.11: Evolution of the fluence of the ordinary (grey), the extraordinary (red) polarized NIR, and the sum-frequency generated (blue) pulse along a $250 \mu \mathrm{m}$ long BBO crystal.

pulse remains moderate. From $50 \mu \mathrm{m}$ to $100 \mu \mathrm{m}$ the parametric amplification process sets in. The intense parts of the near-infrared pulses begin to overlap resulting in an enhanced energy exchange. The SF fluence rises linear and depletion of the fundamentals becomes apparent. From $100 \mu \mathrm{m}$ to $200 \mu \mathrm{m}$ the energy transfer rate stays constant, nevertheless the sumfrequency peak power is still rising. Moreover the overlap region remains constant, i.e. narrow, because of the rapid depletion of the trailing edge of the ordinary pulse and the leading edge of the extraordinary pulse. As a result notable pulse shortening of the SF pulse is observed. The highly

(a)

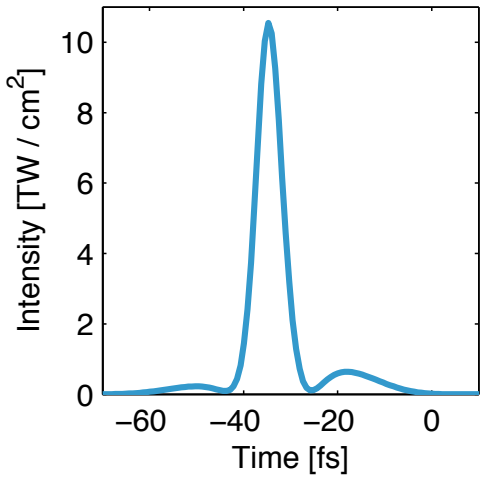

(b)

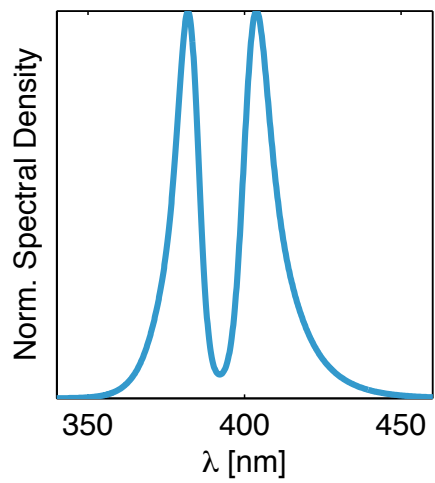

Figure 4.12: Compressed sum-frequency pulse down to 6.4 fs after $250 \mu \mathrm{m}$ of nonlinear pulse compression in BBO, (a) temporal and (b) frequency domain. 
nonlinear interaction in this range is the origin of spectral broadening of the involved pulses. From $200 \mu \mathrm{m}$ to $250 \mu \mathrm{m}$ even more energy is transferred to the SF wave and the SF peak power is still increasing. The pulses are further compressed, but at a much weaker rate. At the same time reconversion from the sum-frequency to the NIR begins to take place. After a propagation length of $250 \mu \mathrm{m}$ in BBO a sum-frequency pulse centred around $390 \mathrm{~nm}$, with a FWHM of $6.4 \mathrm{fs}$, is obtained (see Fig. 4.12). The pulse has a specific double-peaked spectrum, a characteristic feature of nonlinearly compressed pulses.

A part from the SF pulse compression, Fig. 4.10 indicates that the rapid depletion of the trailing edge of the ordinary pulse and the leading edge of the extraordinary pulse can lead to a shortening of the fundamentals. A more detailed analysis about this feature can be found in §4.2.6.

\subsubsection{Shortening of the fundamental frequency pulses}

An interesting feature of the nonlinear pulse compression process is that the ordinary and extraordinary polarized pump pulses can be efficiently compressed if the proper intensity ratio $I_{e} / I_{o}$ is applied. As shown in Fig. 4.13(a), one can control the energy-transfer direction by unbalancing the intensities of the incident pulses. The back-conversion of the ultrashort SF pulse through optical parametric amplification results in fundamental frequency pulse reshaping. If both intensity ratio and pre-delay are ad-

(a)

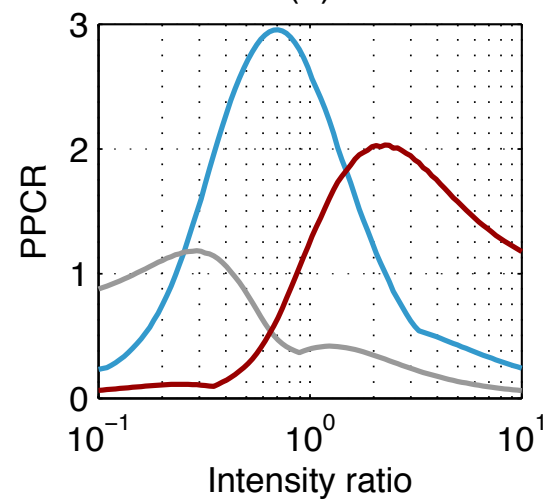

(b)

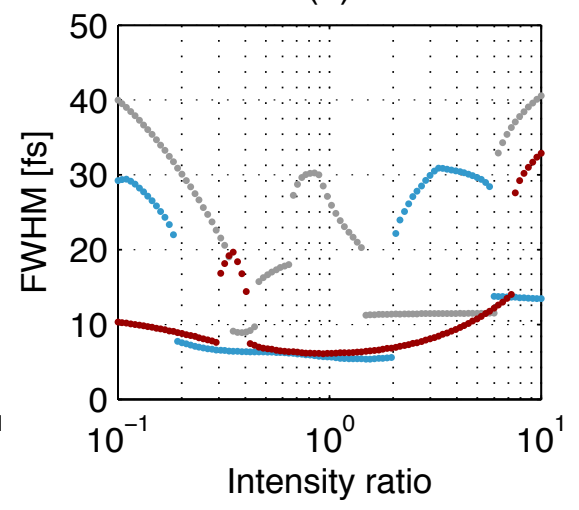

Figure 4.13: Pulse shortening of the extraordinary (red), the ordinary (grey) fundamental frequency pulse, and the sum-frequency generated pulse (blue) as a function of the pump intensity ratio $I_{e} / I_{o}$, (a) peak power conversion rate (PPCR), (b) full width half maximum (FWHM). 
justed in an appropriate way, pulse compression of each of the interacting pulses can be achieved. ${ }^{73}$ Lefort and Barthelemy demonstrated that this effect should be attributed to the second-order cascading process, which can be observed due to unbalanced intensities under exact phasematching conditions. ${ }^{74}$

In the case of $40 \mathrm{fs}$ long NIR pump pulses, with a total incident intensity of $5 \mathrm{TW} / \mathrm{cm}^{2}$, where the ordinary polarized pulse is delayed by $50 \mathrm{fs}$, the peak power conversion ${ }^{2}$ maxima appears at $I_{e} / I_{o}=0.4$ for the ordinary polarized fundamental frequency pulse and at $I_{e} / I_{o}=2.2$ for the extraordinary polarized one. The associated pulses are plotted in Fig. 4.14. The

(a)

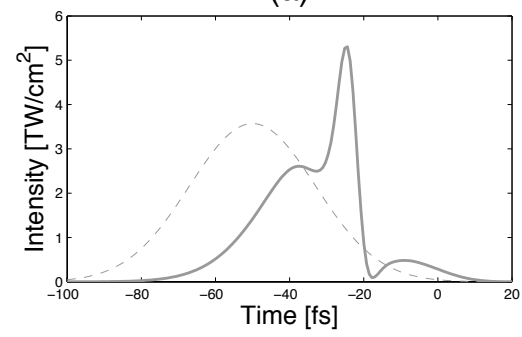

(c)

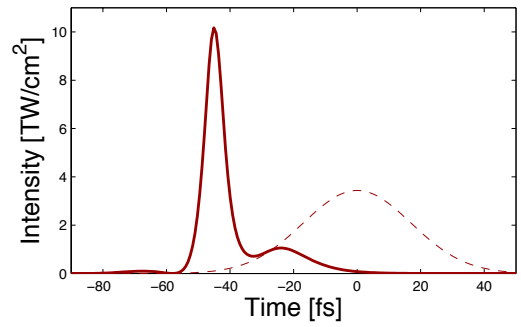

(b)

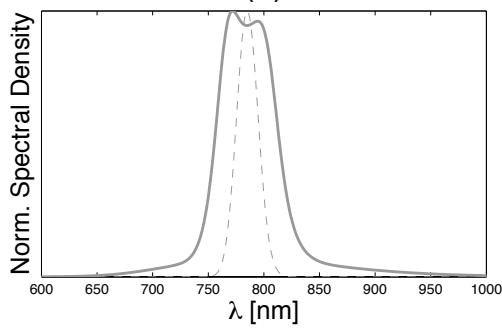

(d)

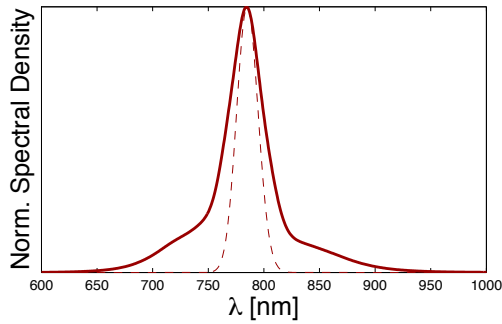

Figure 4.14: Temporal intensity and spectrum of the ordinary polarized NIR pulse for $I_{e} / I_{o}=0.4$, (a) and (b) respectively, and of the extraordinary polarized NIR pulse for $I_{e} / I_{o}=2.2$, (c) and (d) respectively. Initial pulses are drawn as dashed lines.

ordinary polarized pulse can be compressed down to 8.9 fs and the extraordinary down to $7.1 \mathrm{fs}$. Starting with $40 \mathrm{fs}$ fundamental frequency pulses, compression factors exceeding 4 are demonstrated numerically. In general, by changing the pump intensity ratio $I_{e} / I_{o}$, it is possible to compress each of the interacting pulses.

\footnotetext{
${ }^{2}$ The peak power conversion is defined by $I_{i} / I_{i n}$, where $I_{i}$ is the peak power of one of the three waves and $I_{\text {in }}$ is the total intensity of the incident pump pulses.
} 


\subsubsection{Reconstructed pulses}

Using Gaussian pulses as input pulses is a wise approach to study nonlinear pulse compression. The perfect pulses make it easier to distinguish the different features involved in the sum-frequency generation and the influence of the parameters under investigation get more apparent. However, if one wants to get a more accurate representation of the experiment, it is more convenient to use the full reconstruction of a measured pulse as an input, i.e. the spectrum together with the spectral phase. Here I took the full reconstruction of a near-infrared pulse which was measured with the SHG-FROG described in §5.2.1.

In the following comparison between Gaussian and reconstructed input pulses, the same FWHM pulse duration (46 fs) and the same peak power (in total $4 \mathrm{TW} / \mathrm{cm}^{2}$ ) was applied on both pulses. However the structure of the measured pulse is more complex, the phase is not "flat", and most notably the tails are longer. The comparison of the generated sum-frequency spectra (see Fig. 4.15 (a) and (b)) already shows that the difference is quite substantial. For negative pre-delays, the "faster" extraordinary polar-

(a)

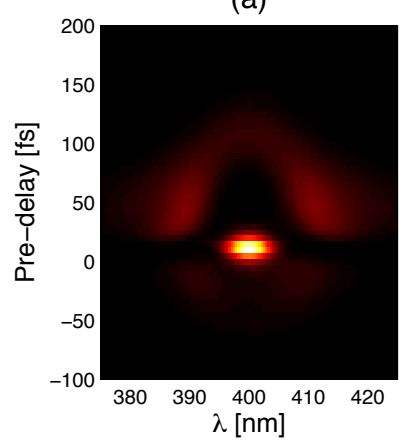

(b)

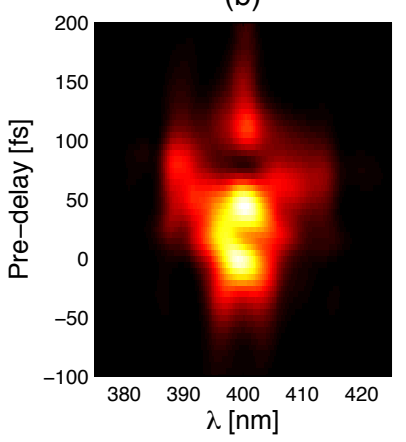

(c)

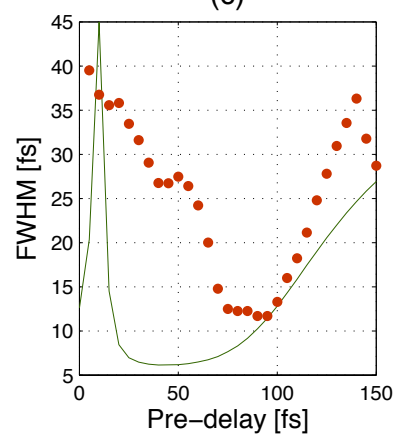

Figure 4.15: Final SF pulse in the frequency domain for initial (a) Gaussian pulses and (b) measured pulses. (c) Respective FWHM pulse durations, Gaussian (line) and measured (red points).

ized pulse enters before the "slower" ordinary polarized pulse into the crystal. In the Gaussian case no sum-frequency components are generated as there is no temporal overlap between the fundamentals. In the reconstructed case some SF frequency components start to appear. The tails of the reconstructed fundamentals are sufficiently long so that the trailing edge of the extraordinary pulse overlaps with the leading edge of the ordinary pulse. The situation is very similar for positive pre-delays bigger than $120 \mathrm{fs}$. Here the leading tail of extraordinary pulse can catch up with the 
trailing tail of the ordinary pulse in the last section of the crystal. For predelays ranging between 25 fs and 90 fs the SFG spectra originating from Gaussian input pulses show the typical double peaked spectrum, whereas the spectra generated with reconstructed pulses show no dip at the centre frequency and the spectral broadening is less prominent. This range is of particular interest as the peak power conversion from the near-infrared to the ultraviolet is moderate here (see also Fig. 4.6).

(a)

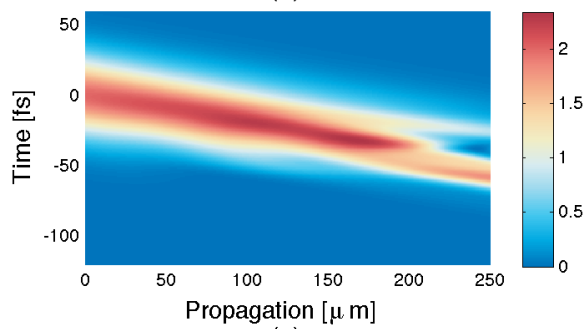

(c)

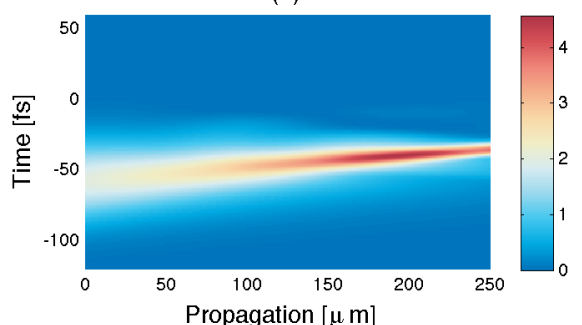

(e)

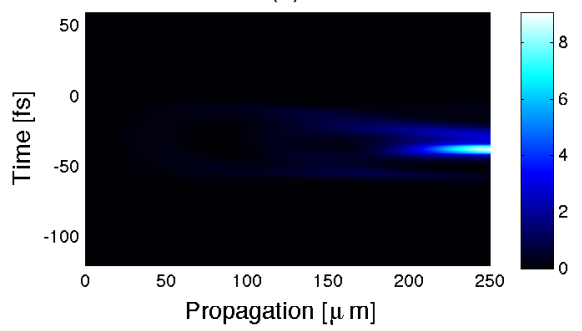

(b)

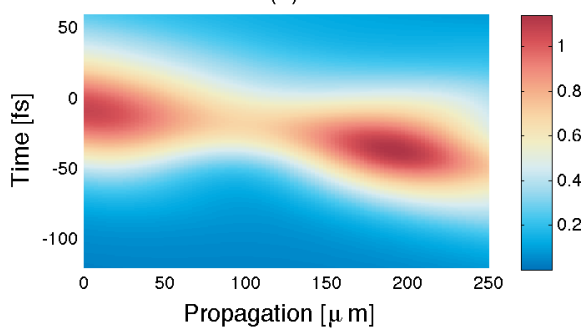

(d)

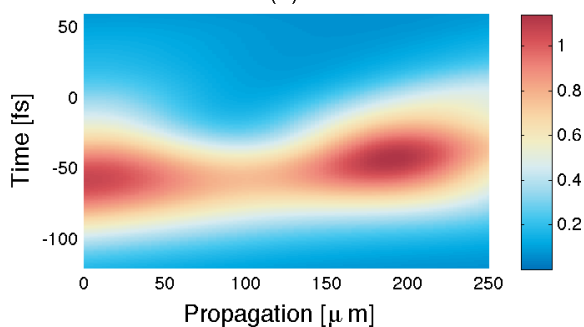

(f)

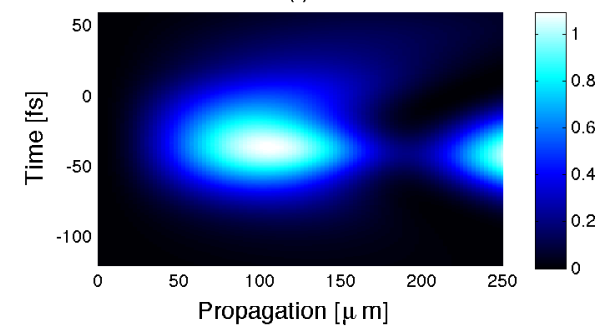

Figure 4.16: Temporal pulse evolution in a $250 \mu \mathrm{m}$ long BBO crystal using (a) extraordinary and (c) ordinary polarized Gaussian input pulses, respectively (b) and (d) reconstructed measured pulses. Resulting SF pulses from (e) Gaussian and (f) reconstructed NIR pulses. All the intensity scales are in $\mathrm{TW} / \mathrm{cm}^{2}$.

To gain more insight I will examine the nonlinear interaction of the three interacting waves for one specific pre-delay: $60 \mathrm{fs}$. The temporal evolution along the BBO crystal over a distance of $250 \mu \mathrm{m}$ is illustrated in Fig. 4.16. The Gaussian near-infrared pump pulses (Fig. 4.16(a) and (c)), as the start 
to overlap sufficiently, get strongly depleted and most of the energy is transferred towards the SF pulse (Fig. 4.16(e)). The temporal overlap region remains small and the resulting SF pulse after $250 \mu \mathrm{m}$ has a pulse duration of $7 \mathrm{fs} F W H M$ with a peak intensity of $\sim 10 \mathrm{TW} / \mathrm{cm}^{2}$. The SF pulse (Fig. 4.16(f)) originating from the reconstructed pulses (Fig. 4.16(b) and (d)) appears earlier, after $\sim 50 \mu \mathrm{m}$ compared to $\sim 100 \mu \mathrm{m}$ in the Gaussian case, and with a significantly broader pulse width. The longer NIR tails overlap earlier and because their intensity is not high enough they are not strongly depleted. The temporal overlap region remains large and thus the SF pulse has a longer pulse duration. After $\sim 100 \mu \mathrm{m}$, strong depletion of the fundamentals together with SFG pulse shortening can be observed. Though, shortly after, most of the energy of the SF wave is back converted to the NIR. During the last fifth of the crystal the energy is again transferred from the NIR to the UV. Due to the reconversion beforehand and the larger intersection of the fundamentals at this point in the BBO, the SFG pulse ends up with a pulse duration three times longer and a peak intensity nearly five times lower compared to the simulation using Gaussian pulse envelopes. Nevertheless very short UV pulses can be generated by increasing the pre-delay (see Fig. 4.15(c)). At pre-delays around 95 fs even the typical double peak spectrum appears. However as the nonlinear interaction distance is reduced, the peak intensities remain 5 times lower compared to the simulation starting with perfect Gaussian pulses. 
CHAPTER 4. NUMERICAL SIMULATIONS 
The operating principle of a Ti:sapphire amplifier laser system with all its components is described in the first part of this chapter. The second part is devoted to the diagnostic techniques used in the present work which allow the measurement of NIR pulses with femtosecond pulse durations and of even shorter nonlinearly compressed pulses in the UV. 


\section{Chapter 5}

\section{Femtosecond laser system and diagnostics}

Many applications in ultrafast optics, e.g., nonlinear pulse compression, require peak powers far exceeding the power levels that can be directly obtained from cw mode-locked oscillators. One very common way to generate such intense femtosecond pulses is to amplify the pulses delivered by a mode-locked laser.

\subsection{Ti:sapphire amplifier laser system}

The name Ti:sapphire refers to the active laser medium; a crystal made of sapphire $\left(\mathrm{Al}_{2} \mathrm{O}_{3}\right)$ doped with titanium ions $\left(\mathrm{Ti}^{3+}\right)$. Ti:sapphire lasers are particularly advantageous for generating femtosecond pulses because of the very large amplification-bandwidth in the near-infrared spanning over $300 \mathrm{~nm}$ (see Fig. 5.1). The absorption band, which peaks near $490 \mathrm{~nm}$, occurs in the blue-green region of the spectrum and makes Ti:sapphire suitable to be pumped by frequency-doubled Nd:YAG lasers, argon-ion lasers, or flash-lamps. The damage threshold of the crystal is very high, in the order of $5 \mathrm{GW} / \mathrm{cm}^{2}$, which allows the generation and amplification of high peak-power pulses.

Here a Ti:sapphire chirped pulse amplification (CPA) laser system is presented delivering millijoule pulses centred around $785 \mathrm{~nm}$ and operating at $\mathrm{kHz}$ repetition rates. 


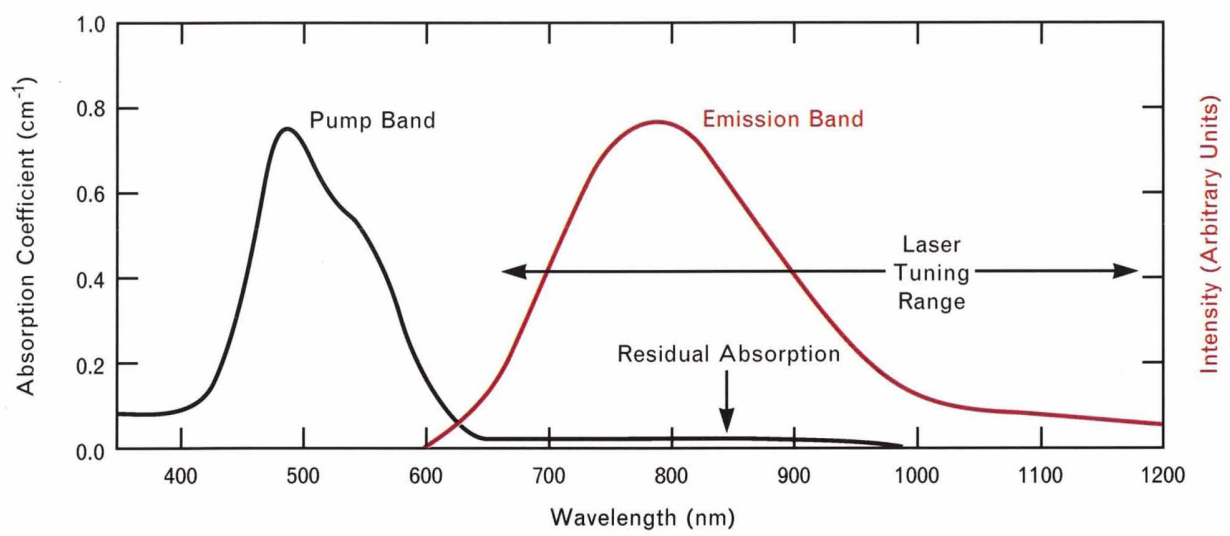

Figure 5.1: The emission and absorption bands of a Ti:sapphire laser crystal. The maximum absorption is around $490 \mathrm{~nm}$ and the emission band peaks near $790 \mathrm{~nm}$. A weak absorption band that overlaps the emission band is known as the residual absorption. Figure taken from. ${ }^{163}$

\subsubsection{Chirped pulse amplification}

When ultrashort laser pulses are amplified, very high optical peak intensities can arise. As a consequence, nonlinear pulse distortions or severe material damage may occur. To overcome this situation D. Strickland et al. ${ }^{164}$ adapted a technique used to increase the available power in radar systems to optical amplifiers. The idea of chirped pulse amplification is to temporal stretch ("chirp") the pulses three to five orders of magnitude before amplification. The stretched pulses have therefore much lower peak intensities and can be safely amplified. Finally, the amplified pulses are recompressed to near their Fourier-transform limit resulting in ultrashort pulses with very high peak powers. A scheme of a typical chirped pulse amplification system is shown in Fig. 5.2.

The accumulated nonlinear phase during propagation in the laser system can be quantified by the so-called B-integral, which is defined as

$$
B=\frac{2 \pi}{\lambda} \int_{0}^{L} n_{2} I(z) d z .
$$

Typically, the system is designed to ensure that $B<1$, otherwise the accumulated nonlinearity can lead to undesired nonlinear effects such as selffocusing, self-phase modulation or filamentation leading to temporal and/or spatial pulse distortions.

The system used in this thesis consists of a commercial femtosecond 


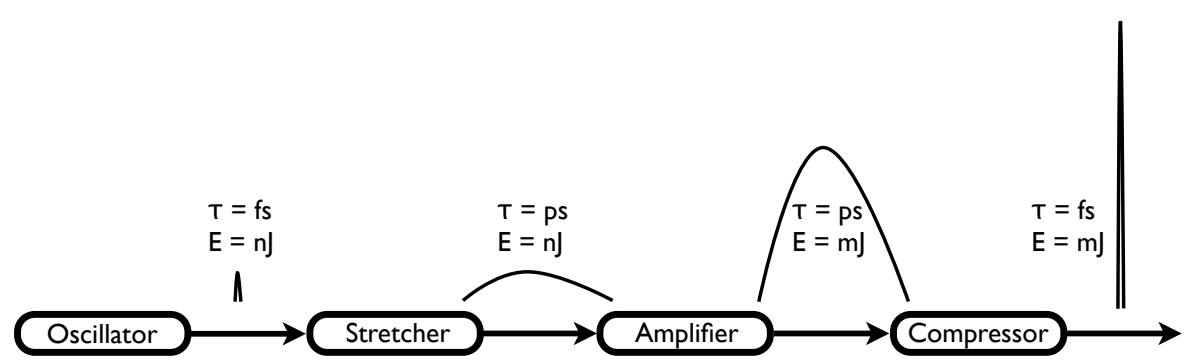

Figure 5.2: Schematic of the CPA process. A pulse is temporally stretched before being amplified and recompressed to near the Fourier transform limit. $E$ refers to the energy level and $\tau$ to the pulse duration.

laser oscillator (Griffin ${ }^{T M}$ KMLabs Inc.) delivering pulses with a duration of $50 \mathrm{fs}$ and energies around $4 \mathrm{~nJ}$. An adjustable grating-stretcher is used to realize the CPA scheme where the pulses are stretched to several hundred picoseconds, amplified in a Ti:sapphire crystal and recompressed again. For amplification of the oscillator pulses two techniques were employed: the multipass and the regenerative amplification. The final stage of the system is a grating compressor.

\subsubsection{Oscillator}

A schematic of the oscillator is shown in Fig. 5.3. The cavity is built around a highly-doped Ti:sapphire crystal, pumped by a $4 \mathrm{~W}$, frequency-doubled, diode-pumped Nd:YVO ${ }_{4}$ Verdi (Coherent). For broadband intra-cavity groupdelay dispersion (GDD) control, a prism pair is used. The oscillator provides a train of $\sim 50 \mathrm{fs}$ pulses at a repetition rate of $78 \mathrm{MHz}$. The spectrum is centred at $785 \mathrm{~nm}$ and has a FWHM of $\sim 100 \mathrm{~nm}$. A typical mode-locked output power of $300 \mathrm{~mW}$ corresponds to an energy per pulse of $\sim 4 \mathrm{~nJ}$.

The laser oscillator generating the femtosecond pulses for the amplifier is based on a Kerr lens mode-locking mechanism. ${ }^{166,167}$ The term modelocking means that there is a phase correlation between the large number of modes oscillating in the laser cavity. The phase-locked modes are preferentially amplified in the cavity resulting in ultrashort pulses with significantly higher intensities than in CW operation, i.e. when there is no phase correlation between the oscillator modes. The intenser pulses induce a Kerr lens in the Ti:sapphire rod, thus undergoing self-focusing. By making the $532 \mathrm{~nm}$ pump focal diameter small in comparison to the CW cavity mode (soft aperture), those pulses experience even more gain, pulsed operation is favoured while CW operation is suppressed. 


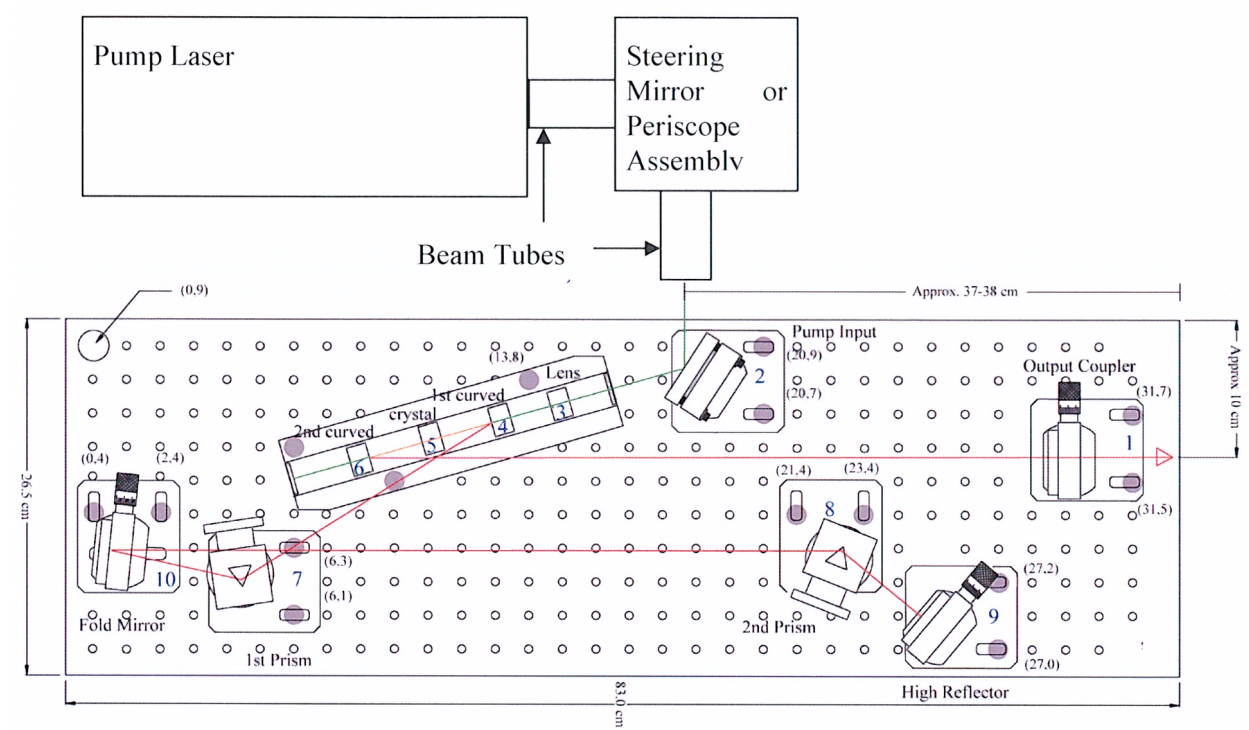

Figure 5.3: Oscillator setup. The Ti:sapphire laser cavity consists of an end mirror (high reflector), folding mirrors at both sides of the crystal, a prism pair and an output coupler. Figure taken from. ${ }^{165}$

\subsubsection{Stretcher}

The grating-based stretcher from KMLabs ${ }^{165}$ makes up the first part of the CPA scheme, which is to produce the temporal broadening of the pulse and reduce the possibility of laser damage to components in the laser amplifier system. Figure 5.4 gives an overhead view of the stretcher layout. The seed beam enters the stretcher reflecting of S-M1 towards S-M2 which sends the beam to the grating. The grating spatially separates the frequency components of the $100 \mathrm{~nm}$ broad oscillator pulse. The spatially dispersed beam is next reflected by a curved mirror to collimate it, folded back upon itself, and reflected off of the grating again. A retro-reflecting mirror sends the beam back through nearly the same path, slightly vertically offset. This time the grating adds all the frequencies back together into the original spatial mode. Slightly different path lengths for the higher (blue) and lower (red) frequencies result in their temporal separation at the output, with the lower frequencies being in front of the higher frequencies. The pulses are now positively chirped. The bandwidth of the stretcher optics is typically around $\sim 150 \mathrm{~nm}$, and it stretches the initial $50 \mathrm{fs}$ long pulse to $\sim 250$ ps. 


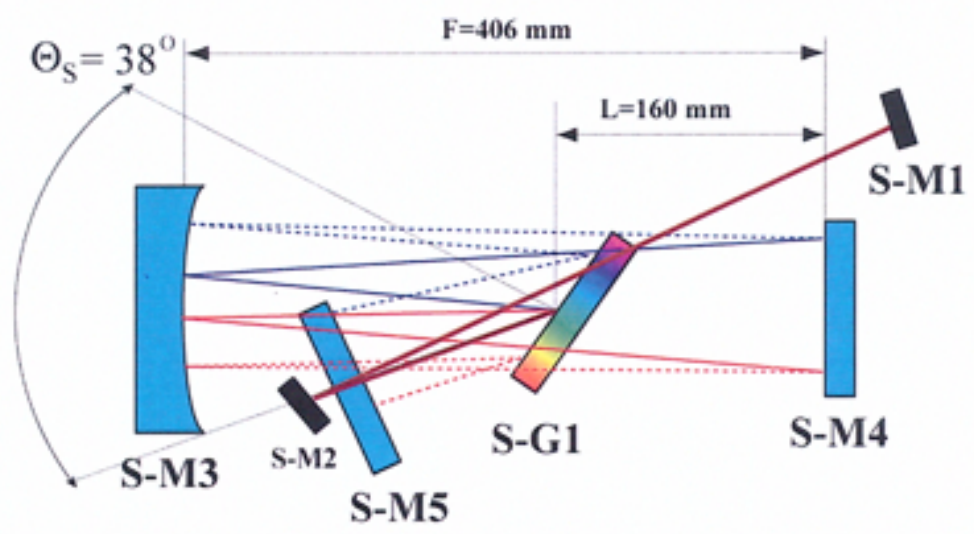

Figure 5.4: Stretcher beam path (view from the top). The solid lines show the beam paths before the fold mirror while the dotted lines the ones after the fold mirror. Figure taken from. ${ }^{165}$

\subsubsection{Amplifier}

The second part of the CPA system consists of generating higher-energy pulses at a lower (kHz-level) repetition rate. First, the stretched oscillator pulses are passed through a combination of a Pockels cell (Bergmann Messgeräte Entwicklung $\mathrm{KG}$ ) and a polarizer to reduce the repetition rate from $78 \mathrm{MHz}$ down to 4 or $3 \mathrm{kHz}$. Second, the pulses are amplified. Two different amplification schemes were employed independently: A multipass amplifier and a regenerative amplifier combined with a power amplifier.

\section{Multipass amplifier}

In our multipass amplification scheme shown in Fig. 5.5, multiple passes of the seed beam are sent through a ring configuration, focusing each pass at the same point within a $7 \mathrm{~mm}$ long Ti:sapphire crystal. The different passes are geometrically separated and the number of passes is therefore limited by the size of the optics and the spot size on them (10 to 12 passes in our case). A mask consisting of an array of holes guarantees proper ring alignment and suppresses amplified spontaneous emission (ASE). The crystal is pumped longitudinally by a frequency doubled, diode-pumped $\mathrm{Nd}$ :YLF $\mathrm{Q}$-switched laser operating at $\mathrm{kHz}$ repetition rates, with an average power of $50 \mathrm{~W}$ (Photonics Industries DM50 Series). To avoid thermal effects such as depolarization loss, thermal lensing or even fracture of the laser crystal, a compact He-gas cryogenic cooling system is used to keep the crystal at $\sim$ $-220^{\circ} \mathrm{C}$ (Cryomech, Cryogenic Refrigerator, AL200). This way much higher 


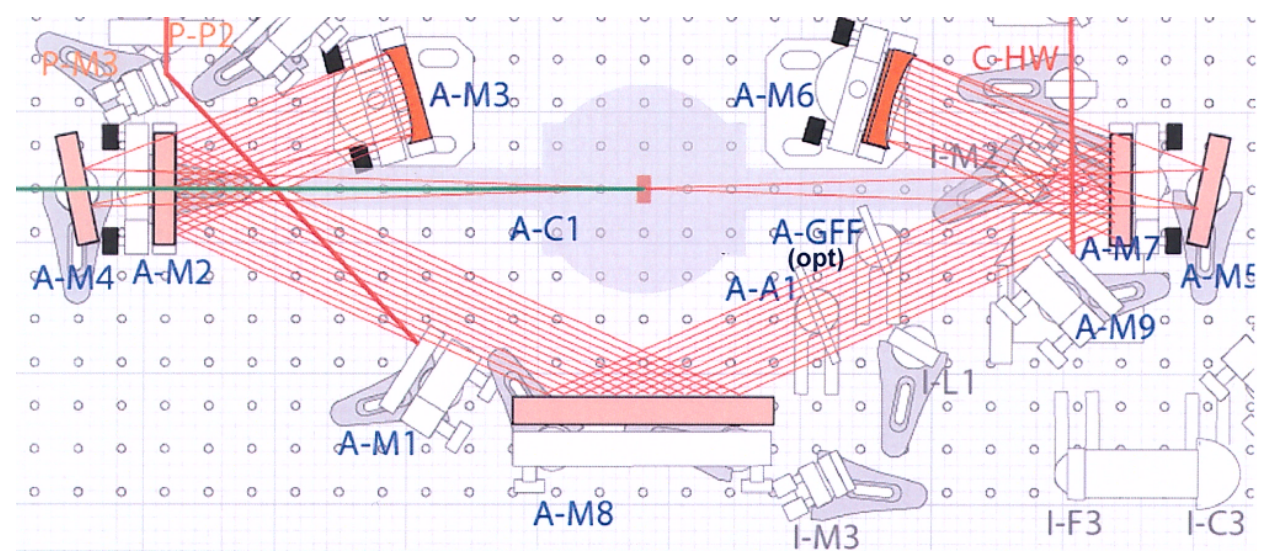

Figure 5.5: Layout of the multipass amplifier ring. Figure taken from. ${ }^{165}$

output powers without excessive thermal effects, i.e. with good beam quality, can be achieved. The crystal is also housed in a vacuum chamber, a turbo pumping station (Pfeiffer, HiCube 80) maintains the chamber at $<10^{-6}$ mbar, to prevent condensation forming on the surface of the cooled crystal which can lead to laser damage. After amplification, the pulses reach an energy of $\sim 2 \mathrm{~mJ}$ before passing to the compressor. This represents an overall gain of $\sim 10^{6}$.

\section{Regenerative amplifier}

In the regenerative amplification scheme, ${ }^{168}$ the oscillator pulse is kept in a laser cavity until all the energy stored in the gain medium is extracted. Injecting and releasing the seed pulses in and out of the cavity is realised by optical switches. The concept of regenerative amplification is illustrated in Fig. 5.6. Here, a Ti:sapphire crystal is first pumped by a frequency doubled, diode-pumped Nd:YLF Q-switched laser. The long energy storage life time of Ti:sapphire $(\sim 3 \mu \mathrm{s})$ allows the pulse to undergo a large number of round trips so that a very high overall amplification factor (gain) can be achieved. The stretched oscillator pulse is injected into the laser cavity through a combination of a thin film polarizer, Pockels cell and a quarter wave plate. After a controlled number of round trips in the cavity, the pulse is extracted by switching the Pockels cell again, causing the pulse to be reflected out of the cavity at the thin film polarizer. Note that this type of amplifier requires a fast switching ( $>3-4 \mathrm{~ns}$ ) Pockels cell as the injection time window must be shorter than the round-trip time. The home-built regenerative amplifier operates at a repetition rate of $4 \mathrm{kHz}$ and is pumped by $15 \mathrm{~W}$ of a pulsed 


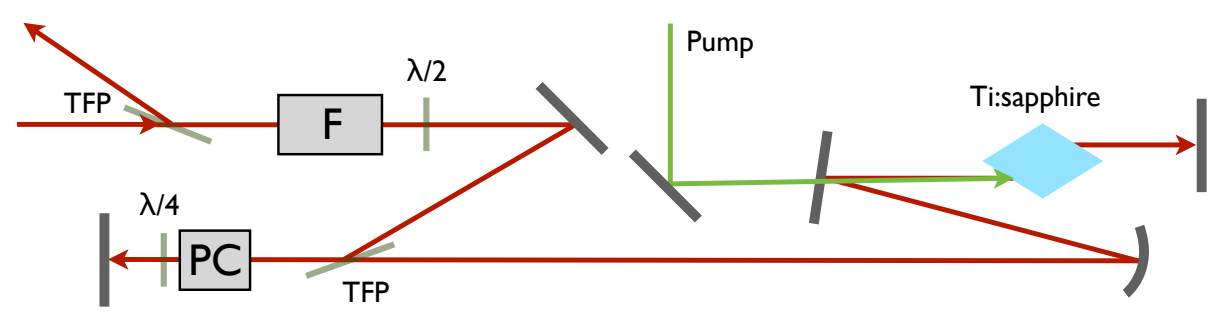

Figure 5.6: Regenerative amplification scheme. A Pockels cell (PC) combined with a quarter-wave plate and a thin-film polarizer (TFP) acts as an optical switch. The Faraday rotator $(F)$ is used for separating input and output pulses.

green laser. In so doing, it is possible to extract $0,5 \mathrm{~mJ}$ pulses with a $40 \mathrm{~nm}$ broad spectrum. The pulses delivered by the regenerative amplifier are further amplified by single passing a cryogenic cooled Ti:sapphire crystal power amplifier pumped by a $50 \mathrm{~W}$ green laser. The obtained laser power is thereby increased by a factor of four.

\subsubsection{Grating compressor}

The third part of the CPA system consists of re-compressing the stretched high-energy pulses. For this purpose a grating compressor is used which negatively chirps the pulses, compensating for the group-velocity dispersion introduced by the stretcher and other system components like lenses and the amplifier crystal. The compressor (illustrated in Fig. 5.7) is composed of two 1200 lines $/ \mathrm{mm}$ diffraction gratings and a pair of mirrors forming a vertical roof-top mirror. The principle of the compressor is very similar to the one of the stretcher described in §5.1.3. The collimated beam hits the first diffracting grating at a certain incident angle. By changing this angle one can control the third order dispersion. The spectrally dispersed beam is then sent to the second grating positioned parallel to the first one. The beam path of the lower frequencies is longer than the one of the higher frequencies and hence by changing the grating separation one can control the second order dispersion in the system. After the second grating the spectral components travel parallel to each other to the roof top mirror which offsets the output beam from the input beam vertically and redirects the beam back to the second grating, inverting the process. When the pulse hits the first grating for the second time the pulse is re-compressed and has its highest intensity, thus determining the minimal beam size in the compressor without causing damage. 


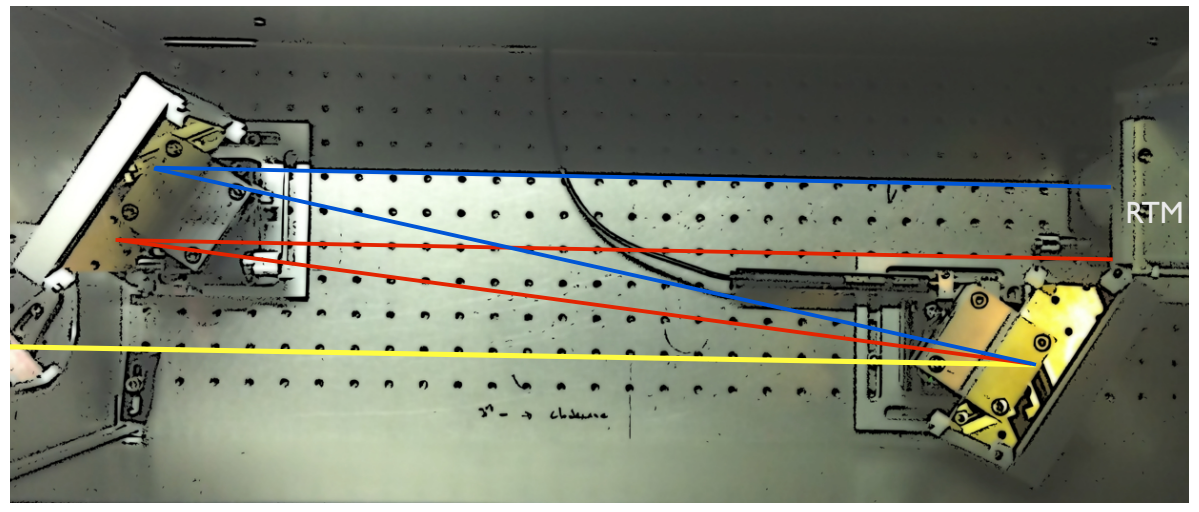

Figure 5.7: Top view of the beam path through the grating compressor. The beam enters from the left (yellow) and is spectrally dispersed after the first grating. After the second grating the frequency components travel parallel to each other. The roof-top mirror vertically displaces the beam and sends it back through the system.

The compressor has an overall efficiency of $>65 \%$ and the 250 ps long amplified pulses can be re-compressed down to $\sim 25$ fs in the case of the multipass amplifier and down to $\sim 40$ fs in the case of the regenerative amplifier.

\subsection{Femtosecond pulse diagnostics}

The full-field characterization of femtosecond laser pulses has always been a challenge since a temporal resolution is needed that is faster than the pulse itself. A popular solution is to use the laser pulse itself as the reference against which it can be measured. The most widely used technique in the ultrafast optics community is the SHG autocorrelator which can be applied through the IR and visible spectrum. However this method does not provide any information about pulse asymmetry because the second-order autocorrelations are symmetric.

In order to fully reconstruct the amplitude and the phase of a pulse an additional measurement is required. By spectrally resolving the autocorrelation signal, a plot of the signal intensity as a function of frequency and delay can be created, which can then be used to completely reconstruct the electric field profile of the pulse through an iterative algorithm. This technique is known as frequency-resolved optical gating (FROG) and exists for several beam geometries, depending on the nonlinear optical effect used for the signal pulse generation, having different strengths and 
weaknesses. ${ }^{169,170}$ A detailed description of the measurement of ultrashort laser pulses using FROG can be found in Trebino's book ${ }^{171}$ devoted entirely to these subject. In the following section only two FROG techniques are introduced: second-harmonic generation FROG (SHG-FROG) and self-diffraction FROG (SD-FROG). While SHG-FROG is the most popular for measuring femtosecond pulses in the NIR, this method is limited at short wavelengths $(\lambda<380 \mathrm{~nm})$ by the UV absorption edge of optical crystals. SD-FROG, however, is a suitable method for measuring ultrashort pulses with spectral components below $400 \mathrm{~nm}$. In general, the FROG technique involves measuring the spectrogram of the pulse given by the following expression:

$$
S(\omega, \tau)=\left|\int_{-\infty}^{\infty} E(t) g(t-\tau) e^{-i \omega t} d t\right|^{2},
$$

where $E(t)$ is the electric field of the pulse and $g(t-\tau)$ is a variable-delay gate function, which is different for each of the various FROG nonlinearities. Knowledge of the spectrogram is sufficient to essentially completely characterize the electric field, except for an absolute phase factor.

Another dominant pulse measurement technique capable of fully reconstructing the electrical field is known as spectral phase interferometry for direct electric-field reconstruction (SPIDER), an implementation of spectral shearing interferometry. ${ }^{172}$ This technique allows the reconstruction of the temporal profile of the pulse directly from the recorded interferogram and hereby allows in principle single-shot measurements.

Finally a commercially available ultrashort pulse measurement system (Phazzler ${ }^{T M}$, FASTLITE) with an acousto-optic programmable dispersion filter (AOPDF) at its core, is presented.

\subsubsection{Second-harmonic generation FROG}

In order to fully characterize the NIR pulses produced by the Ti:sapphire laser, a second-harmonic generation FROG was built. Out of all the different FROG versions, this is the most sensitive one involving only a second order nonlinearity. Using a very thin BBO as a frequency doubling crystal, SHG-FROG is ideally suited for the measurement of broadband nearinfrared pulses and is in principle able to measure the temporal resolution of pulses shorter than $5 \mathrm{fs}$.

A typical setup of SHG-FROG is shown in Fig. 5.8. The pulse to be characterized is split into two replica pulses by a pellicle beamsplitter to 


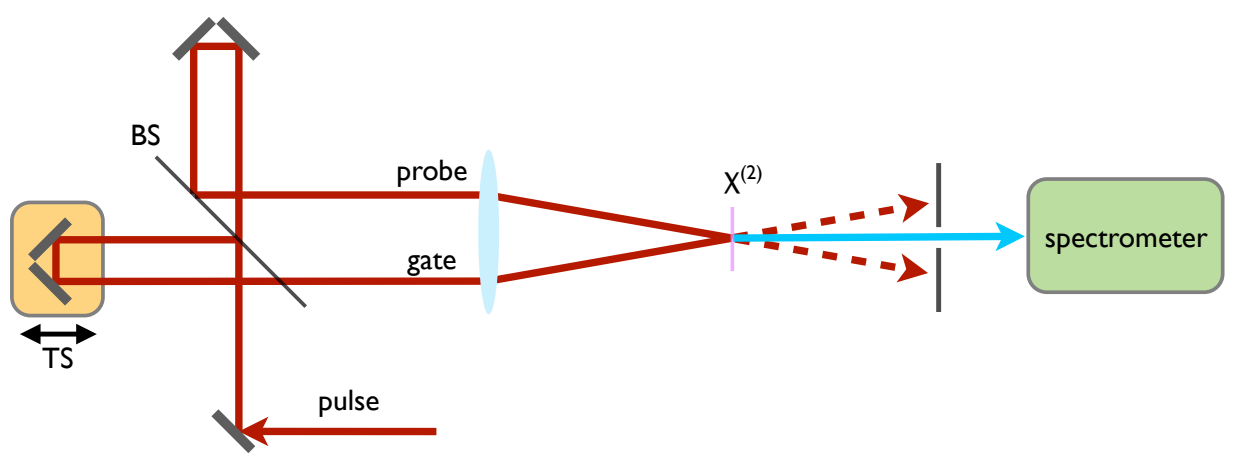

Figure 5.8: A laser pulse is split by a beamsplitter (BS) and one pulse is temporally delayed with respect to the other by passing it through a translation stage (TS). Both pulses are focused into the crystal and the spectrogram $I_{F R O G}^{S H G}(\omega, \tau)$ is generated.

minimize chromatic dispersion. One pulse, the gate $E(t-\tau)$, is variably delayed with respect to the other pulse, the probe $E(t)$, by passing it through a computer controlled motorized translation stage. The two beams are then focused in a nonlinear crystal where the SH signal is generated. An aperture blocks the gate and the probe while the $\mathrm{SH}$ pulse is sent to a spectrometer (HR4000 - Ocean Optics) which spectrally resolves the pulse for each delay. The result is a spectrogram which can be written as follows,

$$
I_{F R O G}^{S H G}(\omega, \tau)=\left|\int_{-\infty}^{\infty} E(t) E(t-\tau) e^{-i \omega t} d t\right|^{2} .
$$

An example of a measured spectrogram, a.k.a. FROG trace, is illustrated in Fig. 5.9(a) where the spectral intensity is displayed on a colour scale as a function of time delay and wavelength (or frequency). The electric field cannot be retrieved directly from the FROG trace, instead an iterative phase retrieval algorithm is used to reconstruct the original pulse by minimising the error between the measured trace (see Fig. 5.9(a)) and the trace generated by the reconstructed pulse (see Fig. 5.9(b)). ${ }^{173}$ The SHGFROG trace should be symmetric about the time axis. As a consequence there is an ambiguity concerning the direction of time which can be removed, e.g., by measuring an additional trace where a dispersive material with a known group-delay dispersion is introduced into the beam path. ${ }^{174}$ Figure 5.9 (c) and (d) finally show the retrieved amplitude and phase in the spectral and the intensity in the temporal domain respectively. The FWHM of the spectrum is $25.6 \mathrm{~nm}$ ) and the pulse duration is $52.9 \mathrm{fs}$ at FWHM. 
(a)

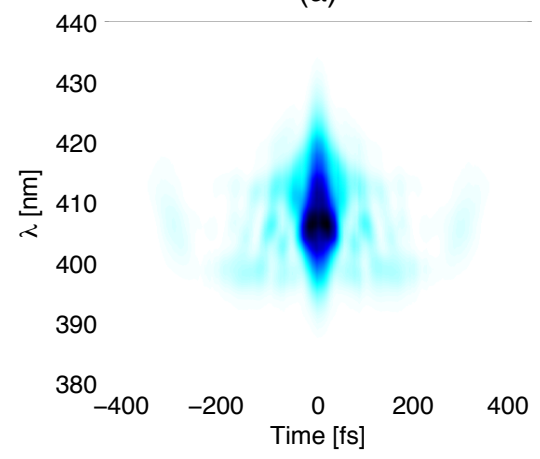

(c)

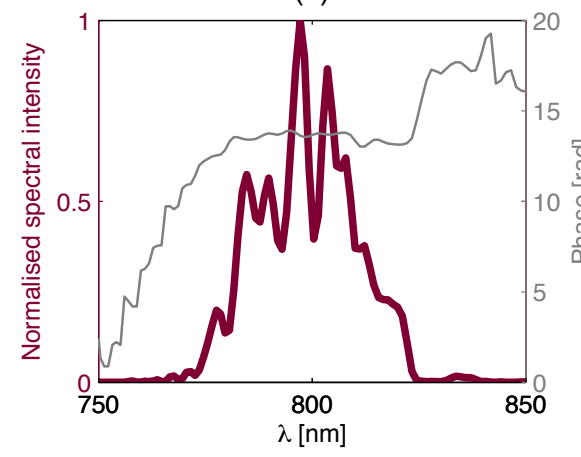

(b)

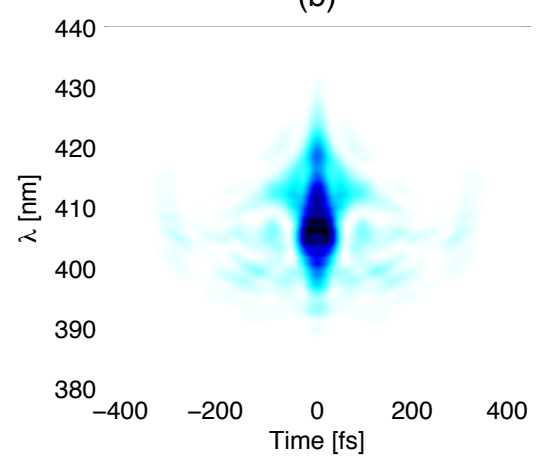

(d)

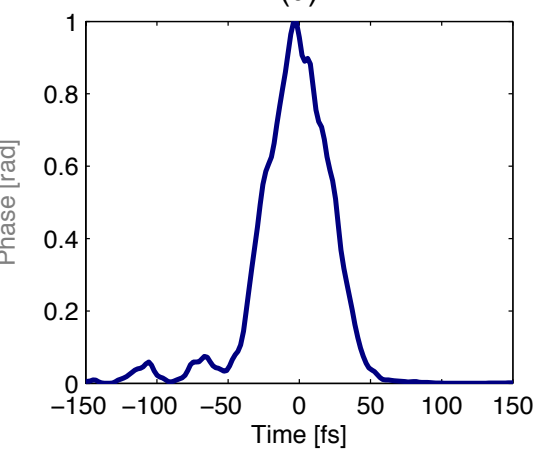

Figure 5.9: (a) Measured and (b) reconstructed FROG trace. (c) Retrieved spectrum (solid red line) and spectral phase (solid grey line), and (d) temporal intensity (solid blue line).

\subsubsection{Self-diffraction FROG}

At shorter wavelengths SHG-FROG is limited by the absorption bandwidth of the frequency doubling crystal, i.e. the reabsorption of the second harmonic. Hence for measuring ultrashort UV pulses it is very difficult to find a suitable crystal. A solution is to use an instantaneous third-order nonlinear process as in self-diffraction FROG (SD-FROG) instead of a second-order one as in SHG-FROG. Besides the capability of measuring ultrashort UV pulses, SD-FROG has no direction-of-time ambiguity and the spectograms are rather intuitive. However, relatively high pulse energies are required; $\sim 10 \mu \mathrm{J}$ for single shot and $\sim 1 \mu \mathrm{J}$ for multishot measurements.

SD-FROG involves crossing the two replicas of the pulse under test within a third order nonlinear material which induces an interference pattern, i.e. a sinusoidal intensity pattern. Due to the intensity dependence of the refractive index, a transient refractive index grating is formed from 
which each replica will diffract (see Fig. 5.10). By spectrally resolving one

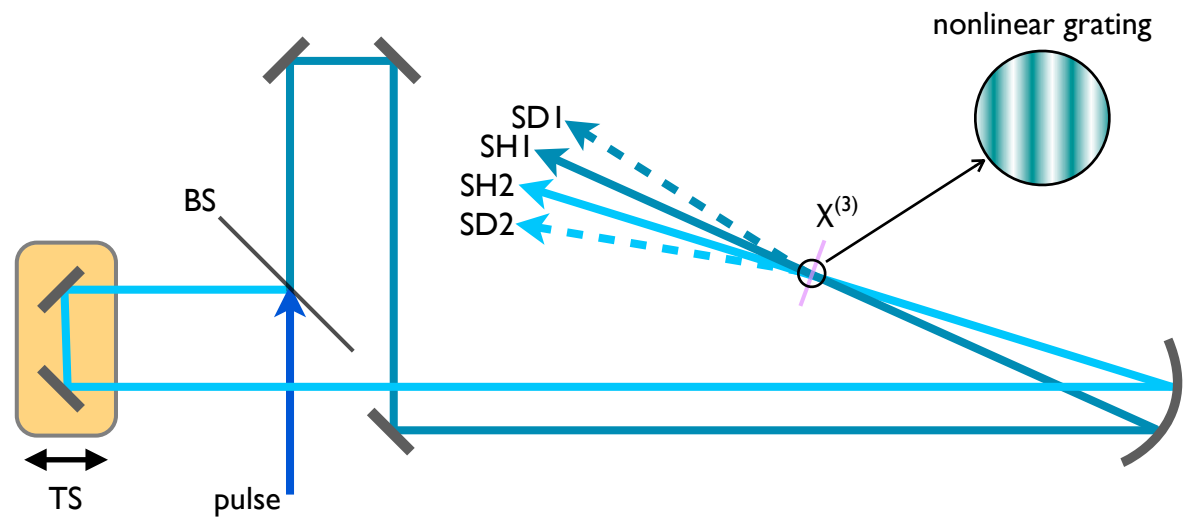

Figure 5.10: Schematic of a SD-FROG setup. BS - Beamsplitter, TS - translation stage. Two beams (SH1 and SH2) are focused within a $\chi^{3}$ medium inducing a nonlinear refractive index grating which diffracts both beams into new beams (SD1 and SD2), one of which is used for detection.

of the diffracted beams and delaying one replica with respect to the other, one obtains a SD-FROG trace which can be expressed as ${ }^{169}$

$$
I_{F R O G}^{S D}(\omega, \tau)=\left|\int_{-\infty}^{\infty} E(t)^{2} E^{*}(t-\tau) e^{-i \omega t} d t\right|^{2} .
$$

As illustrated in Fig. 5.11(a), the SD process involves the interaction of three fields where the direction of the self-diffracted beam is determined by simple vector addition as follows,

$$
\mathbf{k}_{S D}=2 \mathbf{k}_{1}-\mathbf{k}_{2}+\Delta \mathbf{k} .
$$

$\mathbf{k}_{1}, \mathbf{k}_{2}$ are the wavevectors representing the input fields, $\mathbf{k}_{S D}$ represents the $\mathrm{SD}$ field and $\Delta \mathbf{k}$ is the phase mismatch. The phase mismatch is inherent to this process and is an increasing function of the crossing angle between the two incident beams. As a consequence, the angle between the incident beams must be kept small $\left(<2^{\circ}\right)$ in order to minimize the phase mismatch. The phasematching efficiency,

$$
\eta=\left(\frac{\sin \Delta \mathbf{k} L / 2}{\Delta \mathbf{k} L / 2}\right)^{2},
$$

strongly depends on the interaction length $L$ of the nonlinear medium. An example of two phasematching efficiencies is plotted in Fig. 5.11(b) and (c) 
(a)

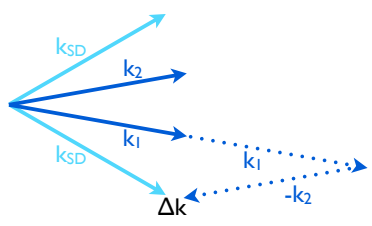

(b)

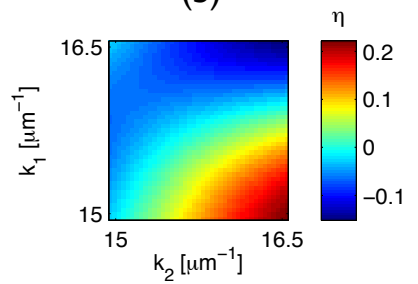

(c)

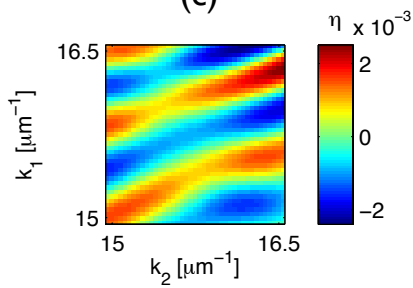

Figure 5.11: (a) Wavevectors involved in the self-diffraction process: $\mathbf{k}_{1}$, $\mathbf{k}_{2}$ represent the input fields, $\mathbf{k}_{S D}$ represents the SD field and $\Delta \mathbf{k}$ is the phase mismatch. (b) Conversion efficiency $\eta$ for a crossing angle of $2^{\circ}$ degree for a $100 \mu \mathrm{m}$ and (c) $500 \mu \mathrm{m}$ thick BBO crystal.

for a nonlinear interaction length of $100 \mu \mathrm{m}$ and $500 \mu \mathrm{m}$, respectively. The crossing angle was chosen to be $2^{\circ}$. The related efficiencies differ by a factor $10^{4}$ which suggests a thin nonlinear medium $(<200 \mu \mathrm{m})$.

SD-FROG traces are highly intuitive as can be clearly seen in Fig. 5.12. Figure 5.12(a) shows the theoretical SD-FROG trace of a Fourier-transformlimited UV pulse with a pulse duration of 20 fs at FWHM. The SD-FROG trace of a dispersion-free pulse is similar to the SHG-FROG trace shown in Fig. 5.9(a). However, the SD-FROG trace becomes asymmetric when some dispersion is added to the pulse as illustrated in Figure 5.12(b). Here we applied $600 \mathrm{fs}^{2}$ of group-delay dispersion (GDD) together with $3000 \mathrm{fs}^{3}$ of third order dispersion (TOD) to the UV pulse. Since the SD-FROG trace is not symmetric about the time origin compared to a SHG-FROG trace, it is very easy to deduce if the measured pulse is positively or negatively chirped. In the case of positive GDD, manifested in the form of a tilted

(a)

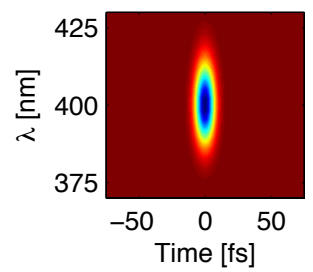

(b)

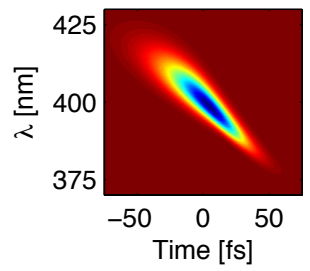

(c)

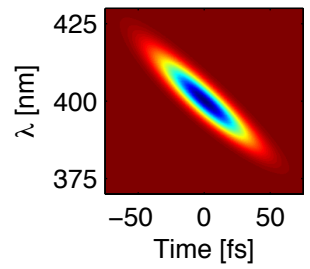

(d)

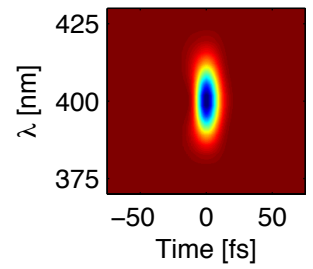

Figure 5.12: SD-FROG traces for a Gaussian pulse with a pulse duration of 20 fs at FWHM and a centre wavelength of $400 \mathrm{~nm}$; (a) Fouriertransform-limited pulse and (b) when material dispersion is added to the pulse. Figures (c) and (d) show the individual contributions of $600 \mathrm{fs}^{2}$ GDD and $6000 \mathrm{fs}^{3}$ TOD respectively. 
ellipse as shown Fig. 5.12(c), lower frequencies (longer wavelengths) are advanced in time ${ }^{1}$ with respect to the higher frequencies (shorter wavelengths). Third order dispersion has the characteristic that high and low frequencies acquire more delay than intermediate frequencies. In the time domain such a pulse has intermediate frequencies in the leading edge and centre but both high and low frequencies in the trailing edge. ${ }^{174}$ SD-FROG traces of a pulse with TOD exhibit a parabolic feature as can be be seen nicely in Fig. 5.12(d).

\subsubsection{SEA-SPIDER}

Spectral phase interferometry for direct electric-field reconstruction (SPI$\mathrm{DER}$ ) is another popular alternative for the complete characterization of ultrashort laser pulses. ${ }^{172,175}$ In contrast to FROG, which is a spectrographic method, SPIDER is based on spectral-shearing, i.e. measures the inter-

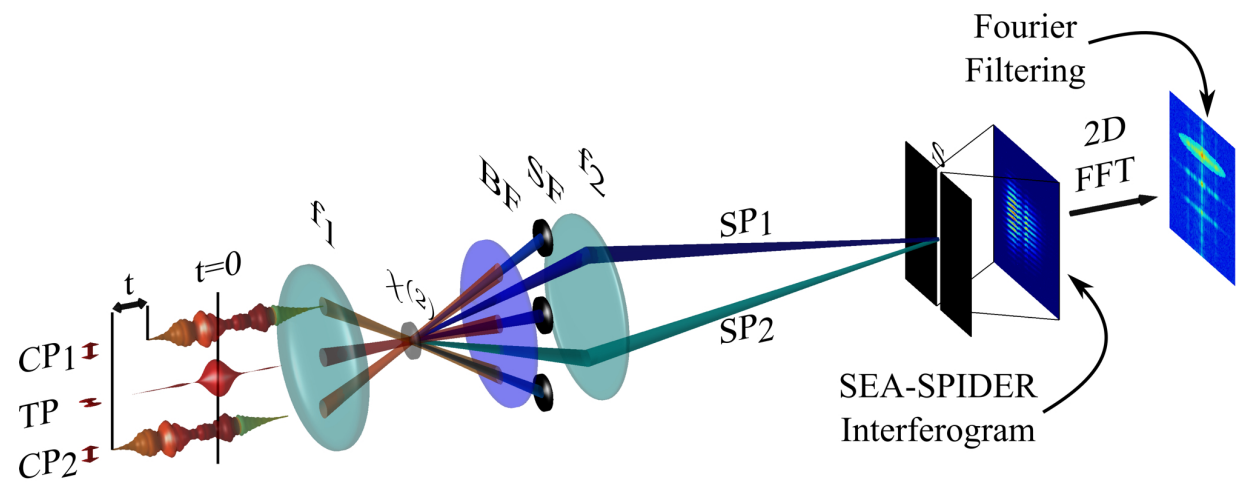

Figure 5.13: SEA-SPIDER concept; TP - test pulse, CP1 \& CP2 chirped pulses, $f_{1} \& f_{2}$ - focusing optics, BF - blue filter, $S F$ - spatial filter, $\mathrm{SP}_{1} \& \mathrm{SP}_{2}-\mathrm{SF}$ pulses. A full description can be found in the text. Figure taken from. ${ }^{35}$

ference spectrum between two spectrally sheared and temporally delayed replicas of the test pulse. The spectral phase, encoded in the interference spectrum, can be retrieved by performing a sequence of Fourier transform, filtering and inverse Fourier transform. Together with the measured spectrum of the test pulse, the electric field can be fully reconstructed. The SPIDER algorithm is much simpler and faster compared to the sophisticated iterative algorithm for retrieving the spectral phase in FROG. The

\footnotetext{
${ }^{1}$ Represented by the negative values on the time axis.
} 
update rates are only limited by the speed of the spectrometer. There are many variants of SPIDER, which have specific advantages under certain circumstances.

Here, a spatially encoded arrangement SPIDER (SEA-SPIDER) device was built with the aim of measuring few-cycle pulses ${ }^{176}$ and the possibility of performing single-shot measurements. The concept is shown in Fig. 5.13: A test pulse is split into two. One of the replicas is strongly chirped by passing it through a $10 \times 3 \times 3 \mathrm{~cm}^{3}$ rod of SF6 and split again. The test pulse is then recombined with the two spatially tilted and highly chirped pulses in a $\chi^{(2)}$ nonlinear crystal (e.g., $30 \mu \mathrm{m}$ type II BBO). During the sum-frequency generation process, the test pulse sees two different quasi-monochromatic frequencies, generating two spectrally sheared replicas of the test pulse centred near twice the fundamental frequency. By

(a)

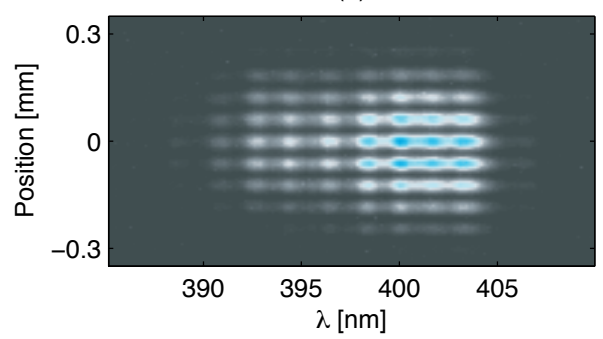

(b)

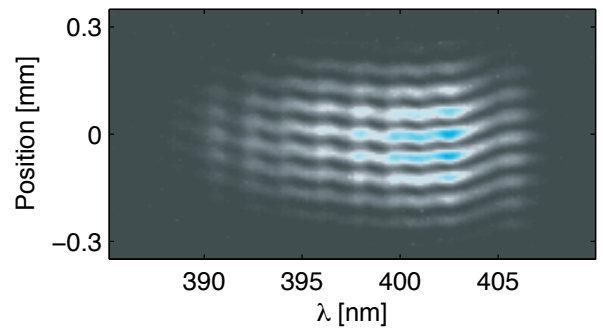

Figure 5.14: SEA-SPIDER interferograms. (a) calibration trace (zero shear) and (b) SEA-SPIDER measurement of a chirped pulse.

applying a temporal delay between the two chirped pulses, one can change the shear independently to the tilt on the beams. After spectral and spatial filtering, the two spectrally sheared replicas are imaged onto the entrance slit of a 2D imaging spectrometer. The tilt between these two pulses results in an interferogram which can be written as

$$
\begin{aligned}
I(\omega, x)= & |E(\omega, x)|^{2}+|E(\omega-\Omega, x)|^{2} \\
& +2|E(\omega, x)||E(\omega-\Omega, x)| \\
& \times \cos [\phi(\omega, x)-\phi(\omega-\Omega, x)+K x],
\end{aligned}
$$

where $I(\omega, x)$ and $\phi(\omega, x)$ are the spectral intensity and spectral phase of the test pulse, $\Omega$ is the spectral shear, $x$ is the spatial position across the beam, $\omega$ is the spectral frequency, and $K$ is the difference in the mean wave vector between the two spectrally sheared arms. The 2D interferograms are very intuitive assuming that there is no space time coupling, 
i.e. the contours of the fringes will directly map the spectral phase gradient. Figure 5.14 shows two interferograms illustrating this intuitive nature. If the delay between the two highly chirped pulses is set to zero, i.e. the shear is set to zero, then the only term left inside the cosine in Eq. 5.7 is $K x$. As this wavelength-independent function only scales linear with $x$, the fringes appear horizontal as can be seen in Fig. 5.14(a). For the final SEASPIDER measurement, see Fig. 5.14(b), a spectral shear is introduced by delaying one highly chirped pulse with respect to the other. A positive

(a) Spectral Intensity/Phase

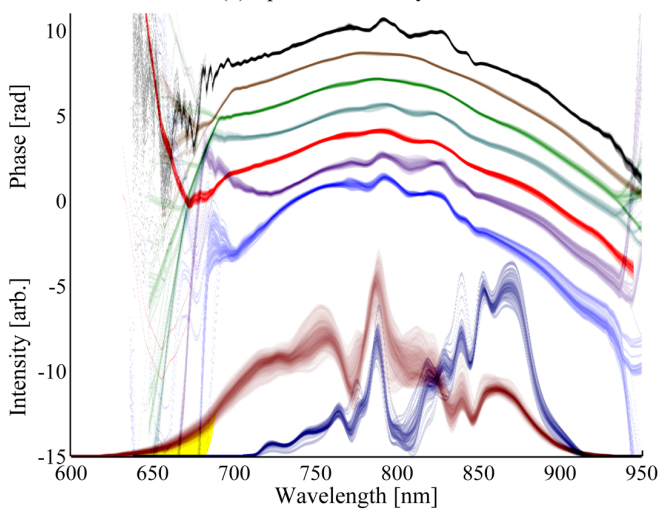

(b) Temporal Intensity

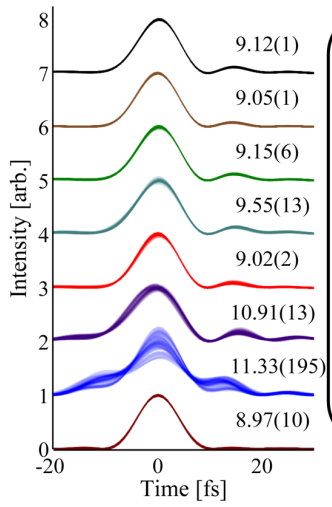

Legend

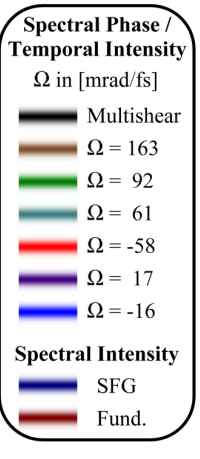

Figure 5.15: Spectral and temporal reconstructions; (a) Spectral intensity and phase. The phases have been offset from each other for clarity. The SFG spectrum has been shifted in wavelength to overlap with the fundamental. (b) Temporal intensity reconstructions and FWHM pulse durations. The Fourier-transform-limited temporal profile (dark red) is calculated from the fundamental spectrum. Figure taken from. ${ }^{35}$

(negative) linear slope of the fringe contours results from positive (negative) group-velocity dispersion respectively, a slight positively (negatively) curvature corresponds to a small positive (negative) third-order phase and small modulations in the fringe contours result from higher-order dispersion. Note that since the pulse is referenced to itself there is no error in the reconstructed phase due to an error in the calibration. ${ }^{177}$ The choice of the shear is a key parameter in spectral-shearing interferometry for fewcycle pulse characterization: it needs to be small enough to sample the fine structure, i.e. rapid phase variations, adequately but also large enough to minimize noise accumulation across the spectrum and to step over any regions of low spectral intensity. Hence, by combining multiple shear measurements, the precision and accuracy of spectral-phase reconstruction 
can be improved. To highlight the advantages of performing spectral shearing with multiple shears, we have performed SEA-SPIDER measurements on a pulse which has been spectrally broadened in an argon filled hollow fibre, but without any external spectral phase compensation. Figure 5.15 shows the spectral intensity and the reconstructed spectral phase for six different shears and their combination. For each spectral shear, 100 SEASPIDER interferograms were taken to enable fluctuations to be measured. The SPIDER phase was then extracted from each interferogram using a slightly modified version of the conventional SPIDER algorithm owing to the two dimensional data set. Instead of performing a one dimensional fast Fourier transform (FFT) filtering routine, one performs a 2D FFT and uses a 2D filter. The two dimensional SEA-SPIDER inversion routine is illustrated graphically in Fig. 5.16. A 2D FFT converts the measured 2D interferogram, Fig. 5.16(a), into the pseudo- $(\tilde{k}, \tilde{t})$ domain with the AC sidebands being shifted to $\tilde{k} \pm K$, Fig. 5.16(b). Note that in Fig. 5.16(b) only the amplitude is plotted, but the complex value is actually retained in the reconstruction. Fig. 5.16. Provided the spatial fringe periodicity is sufficiently

(a)

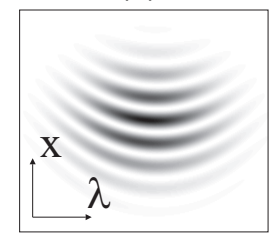

(b)

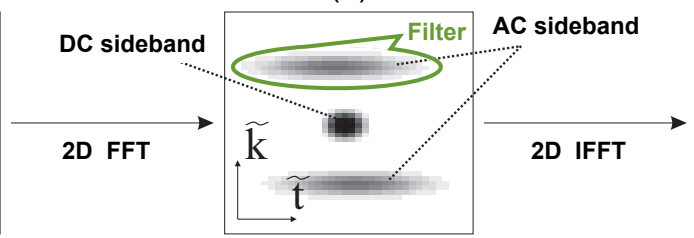

(c)

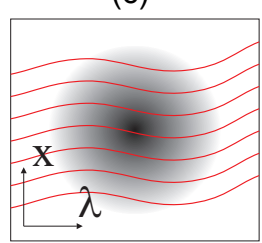

Figure 5.16: Two dimensional SEA-SPIDER inversion routine. (a) The Measured 2D interferogram is converted into (b) the pseudo wavevectortime domain. The filtered data is then inverse Fourier transformed back into (c) the real space-frequency domain, where the phase is extracted and concatenated for each spatial position (red lines); adapted from. ${ }^{178}$

large, one of the $\mathrm{AC}$ sidebands can be selected via a 2D filter. The filtered data is then inverse Fourier transformed (2D IFFT) back into the real- $(x, \lambda)$ domain, where the phase is extracted:

$$
\tilde{\theta}(\omega)=\arg \left\{\mathcal{F}_{2}^{-1}\left[\mathcal{F}_{2}\left[\tilde{I}_{S E A}(\omega, x)\right] f(\tilde{t}, \tilde{k})\right]\right\} .
$$

Before taking the argument, the calibration phase is removed by multiplying the filtered SPIDER data with the complex conjugate of the filtered calibration data. The extracted phase difference is then concatenated along the frequency domain for every spatial position. Compared with conventional SPIDER which does not spatially resolves the spectral phase, SEA- 
SPIDER allows to measure the space-time coupling of the test pulse. The temporal field can then be calculated from the reconstructed spectral phase and an independently measured spectrum.

\subsubsection{Phazzler}

The Phazzler ${ }^{T M}$ (FASTLITE) is a commercially available pulse shaperbased ultrashort pulse measurement system employing a Dazzler ${ }^{T M}$, an acousto-optic programmable dispersion filter (AOPDF) which performs all the linear optical operations on the input pulse such as generating pulse replicas, applying delays between replicas, and managing dispersion. In particular if $\left|\tilde{S}_{i n}(\omega)\right|$ is the input spectral amplitude of an ultrashort laser pulse, the spectral amplitude at the output of an AOPDF, $\left|\tilde{S}_{\text {out }}(\omega)\right|$, can be calculated as follows;

$$
\left|\tilde{E}_{\text {out }}(\omega)\right|=H(\omega)\left|\tilde{E}_{\text {in }}(\omega)\right|,
$$

where $H(\omega)$ is the spectral transfer function. For example, if one wants to create two pulse replicas with a phase offset of $\pi / 2$ and delaying them by $\tau$, then the spectral filter takes the form $H(\omega)=1+e^{j \pi / 2} e^{j\left(\omega-\omega_{0}\right) \tau}$. By changing the spectral filter, i.e. the programmed acoustic wave of the Dazzler, it is possible to reproduce SPIDER, FROG and chirp scan pulse measurement techniques in an in-line geometry without the need of a linear interferometer.

A schematic of the measurement device is sketched in Fig. 5.17. The ultrashort pulse to be measured is sent through a Dazzler AOPDF. Inside the Dazzler the different optical frequencies are diffracted at different longitudinal positions and thus experience different net retardations depending

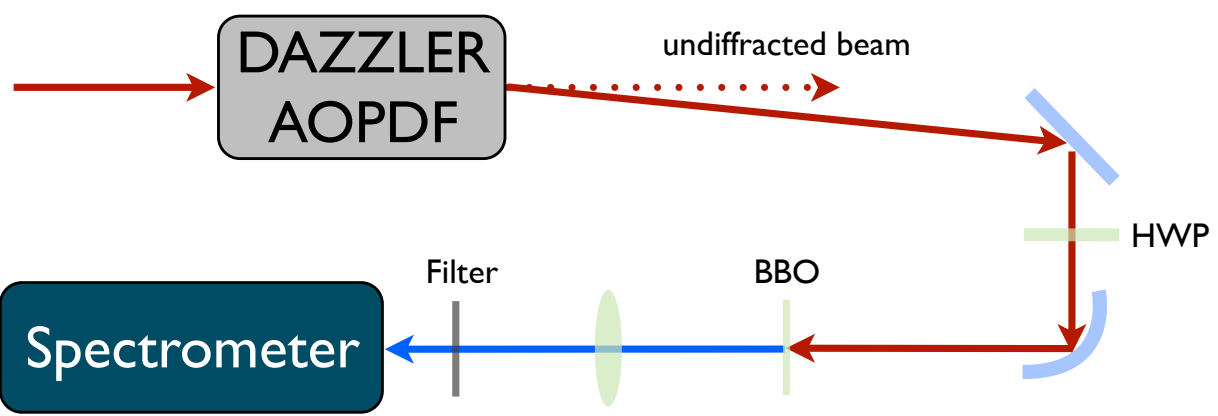

Figure 5.17: Schematic of an AOPDF measurement device; HWP Half-wave plate. 
on the shape of the acoustic wave. A half-wave plate is used to return the diffracted beam to its original polarization required for second-harmonic generation in a type I phasematching BBO crystal. The thickness of the crystal is selected to support phasematching over a large spectral bandwidth. The fundamental frequency is removed with a BG39 filter while the second harmonic is refocused onto a spectrometer (Avantes, 250-550nm, 2048 pixels).

(a)

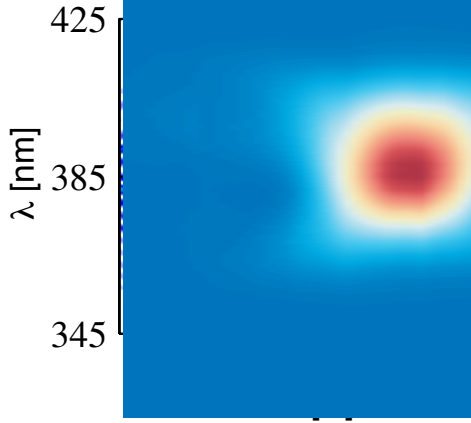

(c)

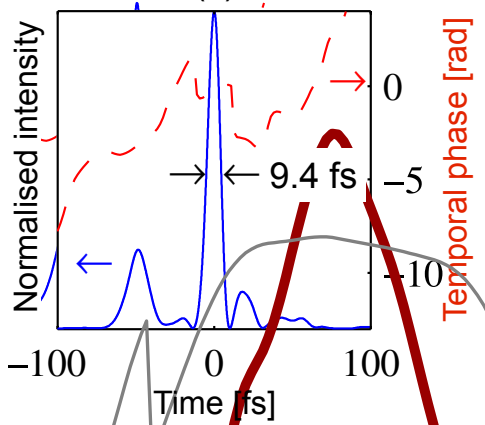

(b)

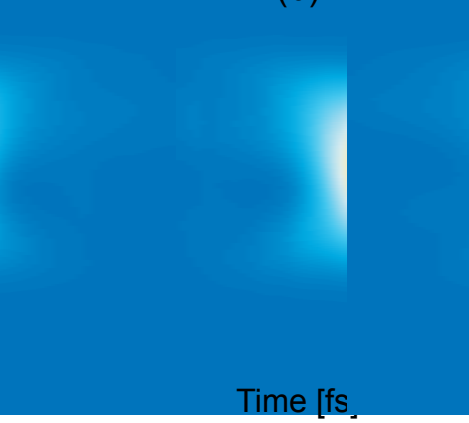

(d)

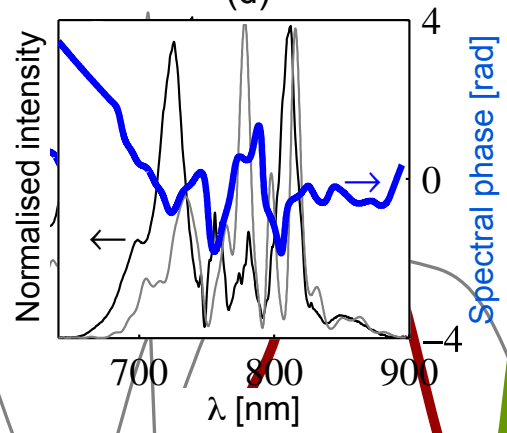

Figure 5.18: (a) Measured bFROG trace, (b) reconstructed bFROG trace, and (c) retrieved temporal intensity shape (FWHM indicated) and phase (red dashed curve). (d) Measured fundamental spectrum (black curve), reconstructed spectrum (gray curve) and spectral phase (blue curve); adapted from. ${ }^{161}$

The AOPDF has a limited range of achievable group delays, $\tau_{\max }$, the Dazzler can compensate ${ }^{2}$ for, fixed by the dispersion $\phi^{2}$ of the birefringent crystal. In our case the Dazzler holds a $25 \mathrm{~mm}$ long $\mathrm{TeO}_{2}$ crystal $\left(\phi^{2} \sim 13000 \mathrm{fs}^{2}\right)$ and can correct for a maximum group delay of $\tau_{\max }=$ 7 ps. This sets an upper limit for the maximum spectral bandwidth the

\footnotetext{
${ }^{2}$ Material dispersion in the crystal requires self-compensation for undispersed output.
} 
Dazzler can operate. The bandwidth limit can be estimated by $\sim \tau_{\max } / \phi^{2}$ which corresponds to $\sim 200 \mathrm{~nm}$ for a square spectrum and to $\sim 80 \mathrm{~nm}$ at FWHM for a Gaussian spectrum, i.e. Gaussian pulses of 20 fs pulse duration. Cousin et. al showed that this measurement technique can be used to characterize few-cycle pulses by using dispersive elements to reduce the Dazzler dispersion. They used a grism pair to precompensate for some of the positive material dispersion of the acousto-optic crystal, extending the range of achievable group delays required for replica production. A grism is a combination of a grating and a prism and a grism pair has the advantage to induce both negative second- and third-order dispersion. ${ }^{179}$ The pulse was measured using the bFROG technique described in. ${ }^{180}$ The measured and the reconstructed bFROG traces, shown in Figure 5.18(a) and Figure 5.18(b), are in close agreement with each other. Figure 5.18(c) illustrates the temporal profile; the duration at full width half minimum from the reconstructed pulse is 9,4 fs pulse with a significant prepulse around $50 \mathrm{fs}$. The reconstructed fundamental spectrum and its spectral phase is shown by the thin gray curve and the thick blue curve in Fig. 5.18(d), respectively, along with the measured spectrum (thin black curves). 
CHAPTER 5. FEMTOSECOND LASER SYSTEM AND DIAGNOSTICS 
Chapter six is dedicated to the generation of ultrashort laser pulses through optical parametric amplification mediated by four-wave mixing. By combining pulses at a center wavelength of $800 \mathrm{~nm}$ and their second harmonic in a gas-filled hollow-core fibre, near-infrared pulses, peaked at $1.4 \mu \mathrm{m}$, with $5 \mu \mathrm{J}$ energy and 45 fs duration at the fibre output were demonstrated. 


\section{Chapter 6}

\section{Near-infrared OPA in a gaseous medium}

Recently, mid-infrared (MIR) generation mediated by the nonlinear Kerr effect was observed in ultrashort laser pulse filamentation pumped by a Ti:sapphire laser source and seeded at the second-harmonic wavelength. ${ }^{82}$ Efficient optical parametric amplification (OPA) has also been demonstrated in gas-filled hollow-core fibres with generation of ultraviolet (UV) light at the third-harmonic wavelength of the $800 \mathrm{~nm}$ Ti:sapphire pump laser. ${ }^{181}$ Gas-filled hollow-core fibres have become a paradigm in the generation of few-cycle pulses at the back-end of commercial Ti:sapphire lasers ${ }^{5}$ as they produce output pulses with excellent mode quality and a uniform temporal pulse profile across the transverse direction.

Here we present results that show optical parametric amplification in a gas-filled hollow-core fibre with the generation of 1-2.5 $\mu \mathrm{m} \mathrm{NIR,} 45$ fs pulses with a peak wavelength at $1.4 \mu \mathrm{m}$ and $5 \mu \mathrm{J}$ energy. Owing to the particular OPA scheme used, we expect these pulses to have constant carrier-envelope phase (CEP). Phasematching of the OPA process is ensured by excitation of higher-order modes as also verified by numerical evaluation of the phasematching relations.

\subsection{Experimental setup}

The experimental layout is shown in Fig. 6.1. The pump pulse was generated by an amplified Ti:sapphire laser and had 30 fs duration, $780 \mathrm{~nm}$ central wavelength. A weak nanojoule level second harmonic seed pulse was created by frequency doubling the pump pulse in a $100 \mu \mathrm{m}$ thick $\beta$ - 


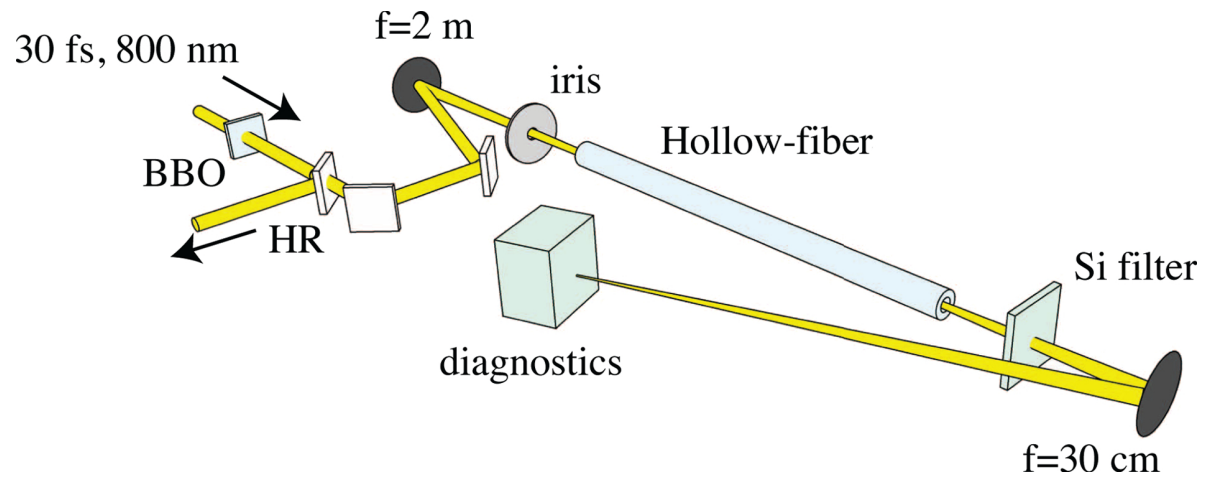

Figure 6.1: Experimental layout; HR - high reflective dichroic mirror. The input Ti:sapphire laser pulse $(780 \mathrm{~nm}, 30 \mathrm{fs}, 1.2 \mathrm{~mJ})$ is frequency doubled in a $100 \mu \mathrm{m}$ thick BBO crystal. The NIR and strongly reduced $\mathrm{SH}$ pulses are focused with a $\mathrm{f}=2 \mathrm{~m}$ focusing mirror into the hollow-core fibre (550 $\mu \mathrm{m}$ inner core diameter, $1 \mathrm{~m}$ length). Figure taken from. ${ }^{143}$

Barium Borate crystal tuned to the optimal phasematching angle ( $\sim 30 \%$ conversion efficiency). The second harmonic was then strongly reduced by a dichroic mirror with high reflectivity at $400 \mathrm{~nm}$. Roughly $1 \mathrm{~nJ}$ of the second harmonic signal leaked through the mirror and co-propagated with the pump pulse. The pump and seed pulses were focused with a $2 \mathrm{~m}$ focal length reflective mirror into a 1 meter long, hollow-core fibre with a $550 \mu \mathrm{m}$ inner diameter filled with argon at 1.4 bar. At approximately $1 \mathrm{~m}$ from the capillary input we placed an adjustable iris. Closing the iris increases the input pump beam diameter at the fibre input and also induces Fresnel diffraction rings: changing these features allowed us to effectively change the coupling efficiency to the various modes of the hollow-core fibre. Finally the output was focused by an $f=30 \mathrm{~cm}$ off-axis parabolic mirror and we then monitored either the spectrum (Ocean Optics spectrometers, HR4000 and NIR256-2.5), the spatial profile (InGaAs complementary metal-oxide semiconductor (CMOS) camera, Xenics, XEVA 202), or the temporal profile with the home-built second-harmonic generation FROG described in §5.2.1.

\subsection{Results}

Figure 6.2 shows the measured spectra under conditions of optimal OPA and NIR pulse generation. The input $800 \mathrm{~nm}$ pulse spectrum, dashed curve in Fig. 6.2(b), is strongly reshaped and blueshifted towards $700 \mathrm{~nm}$, solid 


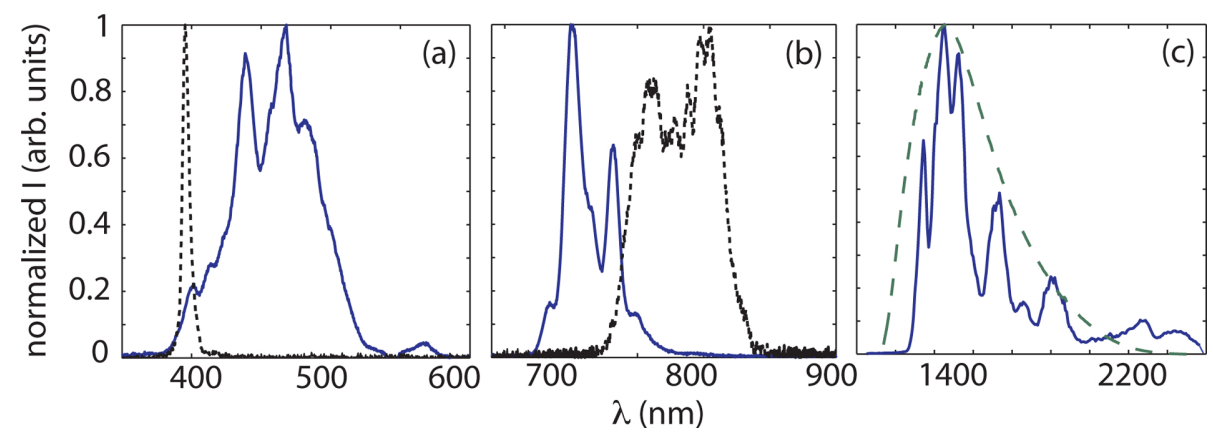

Figure 6.2: Measured spectra. (a) Dashed curve, input second harmonic seed spectrum; solid curve, output amplified seed spectrum. (b) Dashed curve, input pump spectrum; solid curve, output pump spectrum. (c) Solid curve, NIR spectrum with $1 \mathrm{~nJ}$ seed; dashed curve, calculated NIR spectrum starting from the output spectra in (a) and (b). Figure taken from. ${ }^{143}$

curve in Fig. 6.2(b). The reason for this strong blueshift and bandwidth narrowing is most likely related to both a combination of self-phase modulation and plasma defocusing or absorption. ${ }^{182}$ We also observed a similar spectral reshaping, albeit with a smaller shift to $750 \mathrm{~nm}$, when removing the hollow-core fibre and freely focusing in air. The second-harmonic seed spectrum is shown in Fig. 6.2(a). The dashed curve shows the input spectrum while the solid curve shows the amplified and broadened (by cross-phase modulation from the intense pump pulse) spectrum at the fibre output. Changing the iris diameter led to a rather dramatic change in the measured spectrum and output energy: both decreasing and increasing the aperture diameter gives a very strong reduction in the OPA efficiency and the spectrum quickly returns to be very similar to that measured at the input. An optimal aperture of $1.5 \mathrm{~mm}$ was found to give an amplified second-harmonic seed energy of $6 \mu \mathrm{J}$ with a gain factor of 6000 . Finally, Fig. 6.2(c) shows the NIR spectra. The solid curve shows the spectrum for the optimal conditions described above, corresponding to a total NIR energy of $5 \mu \mathrm{J}$, measured after a $3.2 \mathrm{~mm}$-thick silicon filter so as to block the pump and seed pulses. This energy was also optimized by adjusting the argon gas pressure. Lower pressures led to a fast decrease in the OPA efficiency, whereas increasing the pressure above 1.4 bar led to similar, yet slowly decreasing, NIR energies. We note that we also tried to seed the OPA process with the full available second-harmonic energy, i.e., $\sim 100 \mu \mathrm{J}$, which was redirected into the hollow-core fibre after a delay stage and focused with a separate $f=2 \mathrm{~m}$ focal length mirror. Even after 
careful optimization of the delay between the pump and seed pulses, the total NIR energy only increased to $6 \mu \mathrm{J}$. This result seems to imply that the OPA process was already close to saturation with the $1 \mathrm{~nJ}$ seed. Furthermore, we note that with the two-arm setup, a delay variation of less than $30 \mathrm{fs}$ was sufficient to completely suppress all parametric effects. However, the simpler one-arm case shown in Fig. 6.1 did not require a delay stage in order to adjust the relative delay with the pump pulse, and the pulses were automatically synchronized inside the fibre. This is a rather surprising result, considering the fact that propagation in air after the focusing mirror should separate the pump and second-harmonic pulses by more than a pulse width. The reason for the spontaneous synchronization of the two pulses is still not fully clear but may be related to pulse lengthening due to chirp and possibly to dispersion effects in the dichroic mirror coating. The dashed curve in Fig. 6.2(c) corresponds to the spectrum calculated by
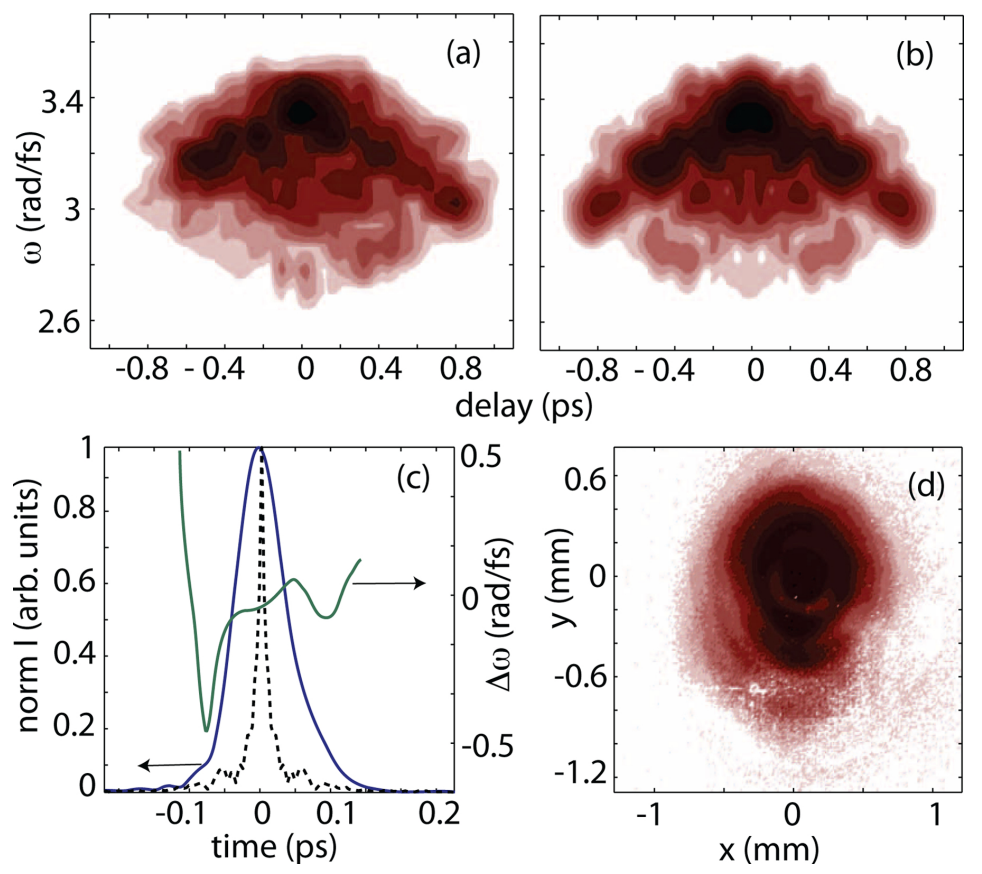

Figure 6.3: NIR temporal and spatial profiles. (a) measured and (b) reconstructed FROG spectrogram (FROG error $0.8 \%$ ). (c) Retrieved temporal intensity with a FWHM of 82 fs (solid blue curve), instantaneous frequency change $\Delta \omega$ (solid green curve), and Fourier transform of the spectrum with a FWHM of 10 fs (dashed curve). (d) Spatial near-field profile of the NIR pulse. Figure taken from. ${ }^{143}$ 
taking all the possible four-wave-mixing combinations, $\omega_{N I R}=\omega_{i}+\omega_{j} \omega_{k}$, where $\omega_{N I R}$ is the NIR frequency and $\omega_{i, j, k}$ are frequencies taken within the pump and second-harmonic spectra as measured at the fibre output. As can be seen, this calculation is able to reproduce both a relatively precise spectral peak wavelength and spectral shape. This supports the origin of the NIR pulse as due to OPA mediated by four-wave-mixing.

Figure 6.3(a) and (b) show the measured and retrieved spectrograms of the NIR pulse obtained by SHG-FROG and Fig. 6.3(c) shows the retrieved temporal intensity profile and the instantaneous frequency variation $(\Delta \omega)$ (solid curves). The FWHM is found to be $82 \mathrm{fs}$. Accounting for the dispersion-induced pulse lengthening in the silicon filter, this corresponds to a 45 fs pulse at the hollow-core fibre output. The dashed curve is the Fourier transform of the total measured spectrum shown in Fig. 6.2(c) assuming a flat phase, indicating that the OPA process is capable of generating 10 fs FWHM pulses. Figure 6.3(d) shows the near-field intensity profile of the NIR pulse measured with an InGaAs CMOS camera. The pulse has a central peak surrounded by a weaker ring, indicating that most of the NIR energy is concentrated in the fibre second- order mode. This higher mode is due to the fact that four-wave mixing is not phasematched if all the pulses are in the fibre lowest-order mode. On the contrary it is possible to find phasematching conditions that give efficient optical parametric amplification by exciting higher-order modes, e.g., with an appropriate input iris aperture.

\subsection{Discussion and conclusion}

We verify this assertion by analytically estimating the conditions for optimal phasematching inside the fibre, i.e. by evaluating the four-wave-mixing coherence length $l_{c}=\pi /|\Delta \beta|$. The phase mismatch $|\Delta \beta|$ is a function of gas pressure, $P$, interacting wavelengths, $\lambda$, and hollow-core fibre radius, $a$,

$$
\Delta \beta=2 \beta_{\text {pump }}-\beta_{\text {seed }}-\beta_{\text {NIR }}
$$

The fibre-mode wave vector is defined as ${ }^{183}$

$$
\beta=\frac{2 \pi n(\lambda, P)}{\lambda}\left\{1-\frac{1}{2}\left[\frac{u_{m, n} \lambda n(\lambda, P)}{2 \pi a}\right]^{2}\right\},
$$

where $u_{m, n}$ is the $n$-th root of the $m$-th Bessel function, $J_{m}\left(u_{m, n}\right)=0$, and $n(\lambda, P)$ is the pressure-dependent refractive index of argon. ${ }^{184}$ Figure 6.4(a) shows the coherence length for a fixed capillary radius $a=$ 

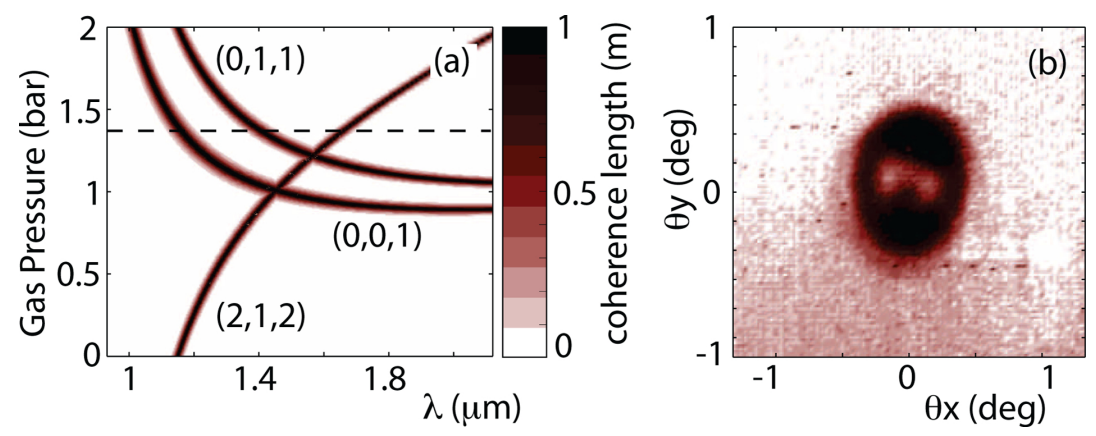

Figure 6.4: (a) Analytically evaluated coherence length for varying argon gas pressure. The three shaded areas show the coherence length for different combinations $(p, q, r)$, where $p, q$, and $r$ are the mode orders of the fundamental, seed, and NIR signal respectively. The dashed curves gives the intersection at the experimentally optimized pressure of 1.4 bar, indicating expected NIR maxima at $\sim 1.3, \sim 1.4$, and $\sim 1.7 \mu \mathrm{m}$. (b) The measured far-field distribution of the NIR mode. Figure taken from. ${ }^{143}$

$225 \mu \mathrm{m}$ and varying gas pressure for three different mode combinations $(p, q, r)$, where $p, q$, and $r$ are the mode orders of the fundamental, seed, and NIR signal respectively. These curves allow us to predict efficient phasematching, i.e. if the coherence length is longer than the fibre length, for wavelengths at $\sim 1.3, \sim 1.4$, and $\sim 1.7 \mu \mathrm{m}$, in close agreement with the peaks in the experimentally measured spectrum (see Fig. 6.2(c)). The important feature here is that the phasematching is ensured through the interaction of higher order modes. As a further confirmation of multimodal propagation and interaction within the fibre we measured the NIR spatial far-field profile, Fig. 6.4(b). The profile shows a clear ring-type shape in keeping with a Bessel-like higher order fibre mode.

In conclusion we have demonstrated efficient OPA in an Ar-filled hollowcore fibre and generation of ultrashort NIR pulses in a setup that is extremely simple. The NIR pulses may either be used directly for nonlinear laser pulse-matter interactions or they may be used as a high-energy seed for improved contrast in $\chi^{2}$ parametric amplification schemes. Finally we note that the four-wave-mixing process involved in the OPA is expected to lead to CEP stable pulses, ${ }^{82}$ a further feature of great importance for applications in extreme nonlinear optics. 
CHAPTER 6. NEAR-INFRARED OPA IN A GASEOUS MEDIUM 


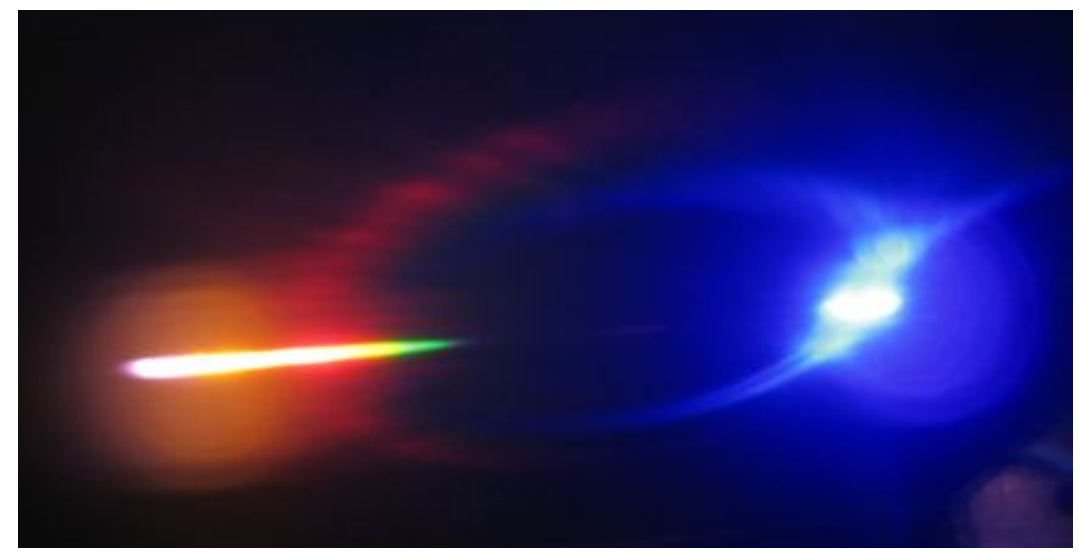

This chapter deals with the nonlinear pulse compression experiment. The first part explains the core principle of noncollinear sum-frequency generation in a type II BBO crystal followed by the description of a very compact setup using transmission gratings and an imaging setup using prisms to induce the pulse-front tilt. At the end of this part the influence of the pulsefront tilt is shown. The second part describes the main experimental results using amplified Ti:sapphire pulses with pulse durations of $74 \mathrm{fs}$ and $46 \mathrm{fs}$. Finally the opportunities for improvement and some limitations concerning this technique are discussed. 


\section{Chapter 7}

\section{Nonlinear pulse compression}

\subsection{Experimental setups}

The nonlinear pulse compression experiments were performed in a type II phasematching $\beta$-Barium Borate (BBO) crystal, by mixing the ordinary and extraordinary polarized pulses from the output of the femtosecond Ti:sapphire amplifier system described in $§ 5.1$. Various setups were employed, all with the aim of fulfilling the appropriate conditions required for nonlinear pulse compression, $v_{o}<v_{S F}<v_{e}$, where $v_{o}$ and $v_{e}$ are the group velocities of the fundamental NIR pulses with the polarization aligned along the ordinary and the extraordinary crystal axis respectively and $v_{S F}$ is the group velocity of the extraordinary polarized sum-frequency generated pulse. The core principle of the noncollinear interaction remains the same for the different setups and is illustrated in Fig. 7.1. The SFG interaction takes place in a $250 \mu \mathrm{m}$ long type II BBO crystal cut at $42.4^{\circ}$ to the optical axis for collinear phasematching around $800 \mathrm{~nm}$. To adjust the desired crystal cut angle $\left(\theta=55^{\circ}\right.$, see Fig. 3.5) we mounted the crystal on a rotation stage. The noncollinear beam geometry allows to control the relative group velocities while maintaining the phasematching condition Eq. $3.30 .{ }^{66}$ For the chosen crystal cut angle, the extraordinary pulse-front tilt outside the BBO has to be $\sim 16^{\circ}$ and the ordinary pulse-front tilt has to be $\sim 18^{\circ}$ to ensure parallel wavefronts inside the BBO. It also ensures that the SFG beam will have its wavefront perpendicular to its propagation vector, i.e. the sum-frequency generated pulse will have no pulse-front tilt.

As discussed in Chap. 3.2, there are different techniques to achieve such significant pulse-front tilts. Out of those, there exist several implementations. Here we investigated two different approaches: (i) a simple setup using gratings and (ii) a more sophisticated one using a combination 


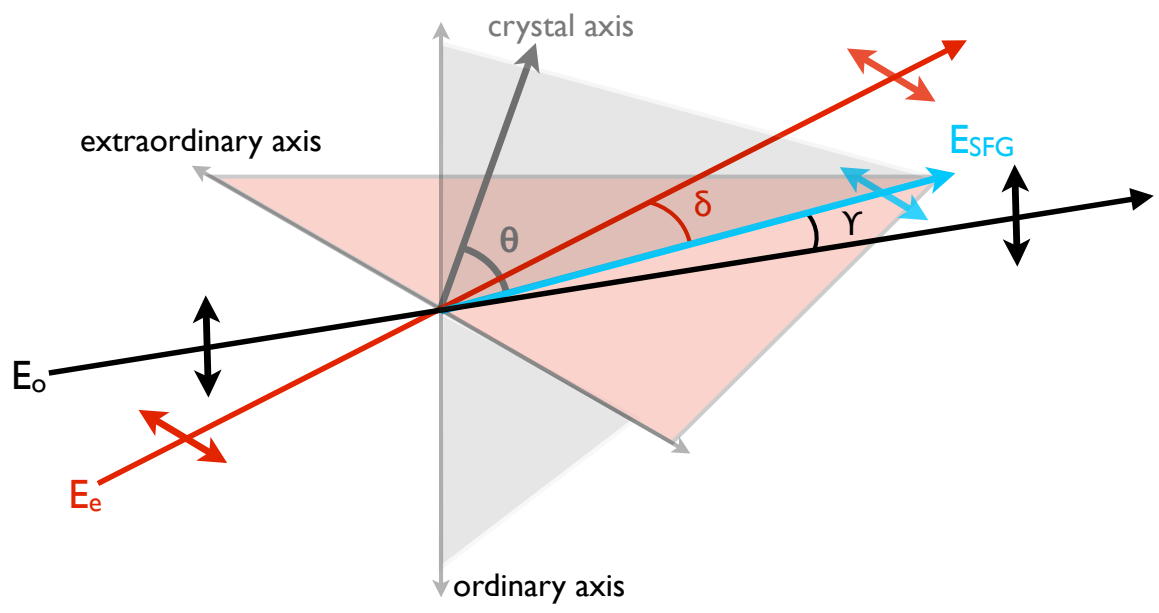

Figure 7.1: Schematic of the noncollinear SFG interaction within a type II BBO crystal, $\theta$ is the crystal cut angle, $\delta$ and $\gamma$ are the noncollinear angles of the extraordinary (red) and the ordinary (black) polarized fundamentals with respect the sum-frequency (blue) generated wave.

of prisms and curved optics. In each setup the NIR pulses delivered by the CPA laser system were separated by a dielectric beam splitter (FEMTOLASERS - OA037) providing a constant splitting ratio of $50 \%$ over a large spectral bandwidth and minimum GDD upon both reflection and transmission. The pre-delay could be controlled by a stepper motor actuator (Thorlabs - Z825B) mounted on a translation stage which was placed in one arm. The minimum incremental movement which could be achieved with the actuator is $0.05 \mu \mathrm{m}$ corresponding to $\sim 167$ as. In order to turn the polarization direction by $90^{\circ}$, a broadband half-wave plate centred at $800 \mathrm{~nm}$ (Tower Optical Corporation) was placed in the reflective path of the beam splitter. The GDD of the $1 \mathrm{~mm}$ thick beam splitter substrate balances approximately the GDD of the retardation plate made out of crystal quartz.

\section{Grating based setup}

First, we built a setup using NIR transmission gratings with a groove density of 300 lines $/ \mathrm{mm}$ (Thorlabs - GTI25-03). A schematic of the main part of the experimental setup is shown in Fig. 7.2. The setup is very compact and relatively easy to align. Care has to be taken with the position of the gratings. The angular dispersion induced by the gratings introduces a tem- 


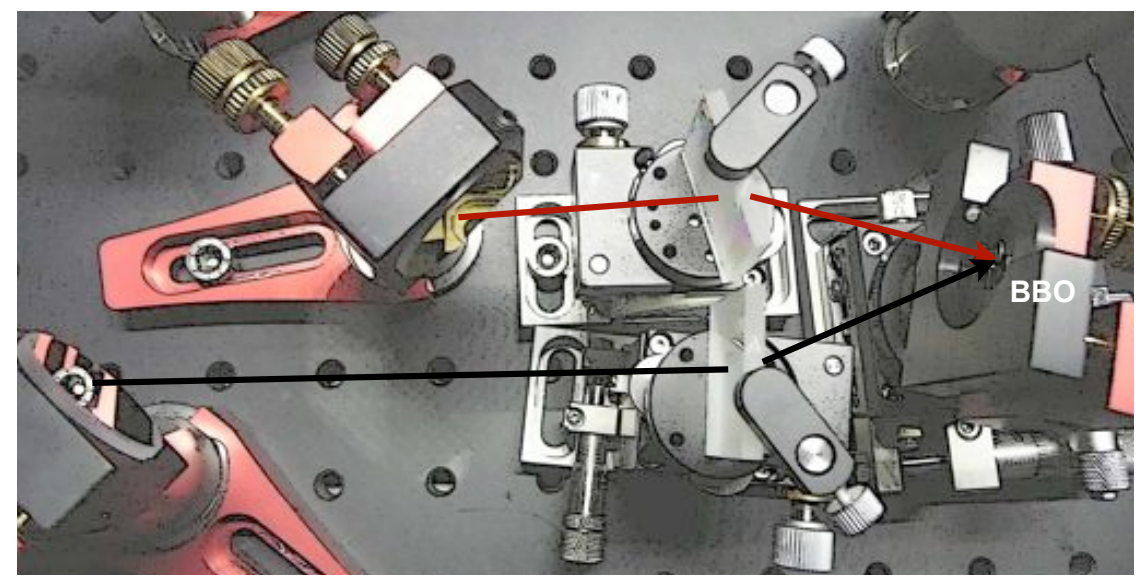

Figure 7.2: Compact setup using transmission gratings.

poral negative chirp and at the same time a spatial chirp. The temporal linear chirp that an ultrashort pulse can acquire from angular dispersion can be expressed in the simple plane-wave model ${ }^{185}$ as

$$
\phi^{(2)}=-k \beta^{2} z / 2,
$$

where $k=2 \pi / \lambda$ is the wave number, $\beta$ is the angle of the pulse-front tilt and $z$ is the propagation distance. For example, a grating-to-crystal distance of $5 \mathrm{~cm}$ would introduce a negative dispersion of $15312 \mathrm{fs}^{2}$, assuming a pulse centred at $800 \mathrm{~nm}$ and a pulse-front tilt of $\sim 16^{\circ}$. This amount of dispersion can in principle be compensated by reducing the grating separation in the compressor from the CPA laser system by approximately $4.2 \mathrm{~mm}$, but only if the distances of the transmission gratings to the crystal are equal. Unequal distances would lead to different initial pulse lengths at the interaction plane. The dispersion of the different wavelength components results in a lateral broadening of the beam profile and in the worst case in a complete separation of the wavelength components and hence in a decreasing peak intensity with increasing distance. Since very high intensities (up to a few $\mathrm{TW} / \mathrm{cm}^{2}$ ) are needed for nonlinear pulse compression, the distance between the gratings and the crystal should be as short as possible. On the other hand making the grating to BBO distance shorter increases the risk of laser induced damage threshold (LIDT) in the grating substrate. If one assumes a 35 fs long Gaussian pulse at the crystal together with the conditions mentioned in the example above, the pulse duration at the gratings would be $1 \mathrm{ps}$. As the intensity scales inversely proportional with the pulse duration, this corresponds to an intensity decrease of a factor 30 at the grating plane, without taking into account the broadening of the beam profile. 
However, a factor 30 is not enough as the damage threshold of the gratings (Schott B270 10 GW/cm ${ }^{2}$ ) is well below the LIDT of BBO $\left(>3.5 \mathrm{TW} / \mathrm{cm}^{2}\right.$ ) and before all well below the intensity threshold needed for nonlinear pulse compression of femtosecond pulses. Unfortunately no compromise can be found for ultrashort NIR pulses. If the distance between the gratings and the BBO is too short there is a very high risk that the gratings get damaged, while at a grating-safe distance the lateral broadening of the beam profile becomes too significant.

Although the necessary intensities for compressing femtosecond pulses with this very compact optical setup cannot be reached, it should be suitable for the compression of picosecond pulses. Furthermore it serves very well as a proof of principle experiment showing that the phasematching calculations of the pulse-front tilts in a noncollinear geometry are correct.

\section{Prism-telescope based setup}

Another way to introduce the necessary pulse-front tilt is to use a prism in combination with a downsizing telescope. The pulse-front tilt one can usually expect from a prism lies between 2 and 10 degrees, depending on the incident angle and on the material of the prism. The magnification of the telescope then induces an increase of the pulse-front tilt by the same amount. The big advantage of this optical arrangement is that the incident intensity at the crystal scales with the square of the magnification. For example, for a required pulse-front tilt of $18^{\circ}$, a prism which introduces a $3^{\circ}$ tilt, and a magnification of $1 / 6$ results in an increase of the intensity by a factor 36 compared to the intensity at the prism. At the same time the pulse duration is the same at the image (crystal) and object (prism) plane, only the dispersion caused by the prism has to be taken into account. Another advantage is that the beam profile doesn't suffer from lateral broadening.

An implementation of such a setup is illustrated in Fig. 7.3. The incoming $\pi$-polarized Ti:sapphire pulse is split by a 50:50 beamsplitter. The reflective part (o-ray) is sent through a delay stage and a half wave plate which was placed after the prism ${ }^{1}$. The transmitted beam (e-ray) is directly sent through a prism. The equilateral prisms (Thorlabs 853 ) are made out of crown glass (F2), introducing a pulse-front tilt of $\sim 2.6^{\circ}$ and $\sim 3^{\circ}$ at incident prism angles of $60^{\circ}$ and $55^{\circ}$ to the ordinary and the extraordinary pulse respectively. We used protected reflective mirrors instead of lenses to avoid unwanted nonlinear effects. The two concave mirrors $(r=$

\footnotetext{
${ }^{1}$ According to Snell's law, the transmission of the $\pi$-polarized pulse is significantly higher than the $\sigma$-polarized one.
} 


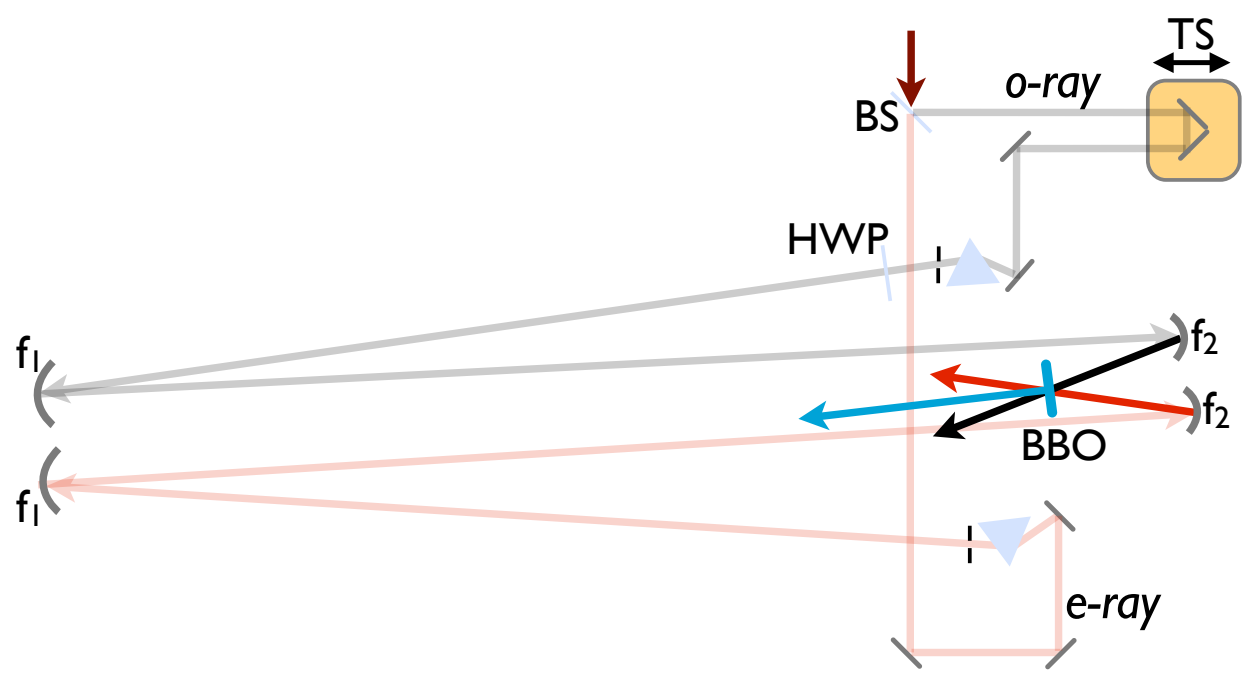

Figure 7.3: Layout of the nonlinear pulse compression setup; BS beamsplitter, TS - translation stage, HWP - half-wave plate, $\mathrm{f}_{i}$ - curved mirrors.

$-1500 \mathrm{~mm}$ and $\mathrm{r}=-250 \mathrm{~mm}$ ), forming the downsizing telescope, provide a magnification of $1 / 6$. The prism-telescope finally induces a PFT of $\sim 16^{\circ}$ on the $\sigma$-polarized pulse and $\sim 18^{\circ}$ on the $\pi$-polarized pulse outside the BBO crystal (see Fig. 3.10(b)) which translates to a pulse-front tilt of $\sim 9.5^{\circ}$ and $\sim 10^{\circ}$ respectively inside the crystal. The crystal is oriented in such a way that the ordinary axis aligns with the $\sigma$-polarized wave. A longer path, i.e. increasing delay, corresponds then to a later arrival time of the ordinary aligned pulse. As the group velocity of the ordinary wave in BBO is inferior compared to the extraordinary wave, this path should always be shorter than the path of the $\sigma$-polarized pulse.

The dynamics of nonlinear pulse compression are very intensity sensitive. High intensities lead to short SF pulse durations whereas lower intensities lead to longer SF pulse durations. Since the final pulse duration is an integral over the whole beam, in the case of a Gaussian beam profile the shortest pulse will be probably blurred out by the components originating from the lower intensity tails, thus a homogeneous flat top beam profile is desired. In order to reduce big intensity variations across the beam cross section, an iris was placed into each beam path directly after the prisms, the object plane with respect to the crystal. This way intensity variations of less than $30 \%$ over the whole beam could be achieved. The exact intensity was determined by taking an image with a high sensitivity CMOS camera 
(a)

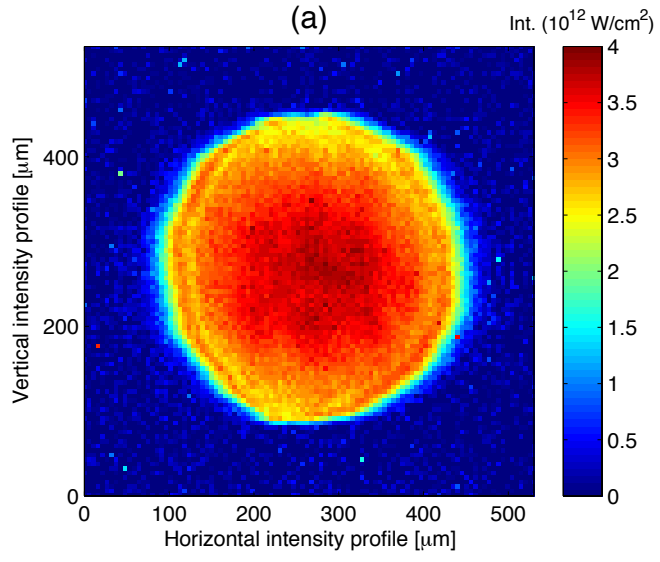

(b)

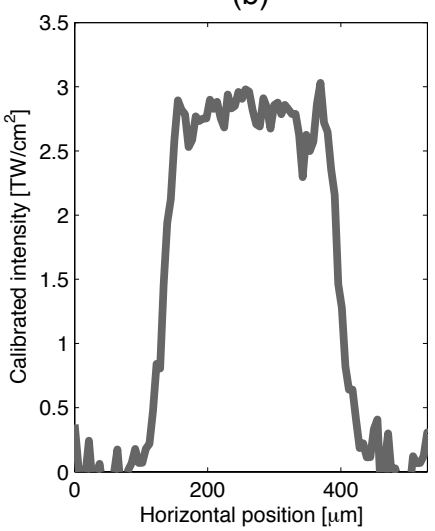

Figure 7.4: (a) Intensity calibration of the spatial beam profile. (b) Horizontal beam cross section.

in the crystal plane (Thorlabs DCC1240C). By measuring the power, knowing the pulse duration, the repetition rate, and the pixel size $\left(5.3 \times 5.3 \mu \mathrm{m}^{2}\right)$, it is possible to get an accurate intensity calibrated beam profile as shown in Fig. 7.4. Here the diameter of the iris aperture was $2 \mathrm{~mm}$, the measured average power was $600 \mathrm{~mW}$, the pulse duration was $46 \mathrm{fs}$ and the repetition rate of the laser was $4 \mathrm{kHz}$.

We also took a caustic around the crystal plane to ensure a very good spatial overlap (see Fig. 7.5). The beam profile of the $\pi$ - and $\sigma$-polarized pulses were taken from $2 \mathrm{~mm}$ before to $2 \mathrm{~mm}$ after the crystal plane in steps of $1 \mathrm{~mm}$.

(a)

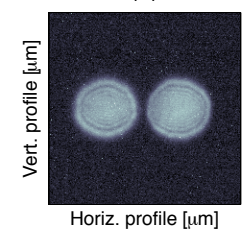

(b)

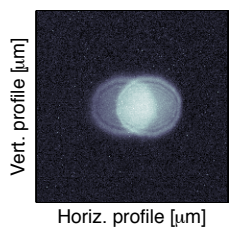

(c)

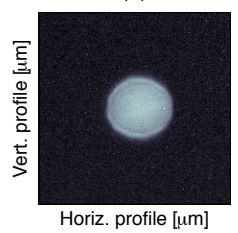

(d)

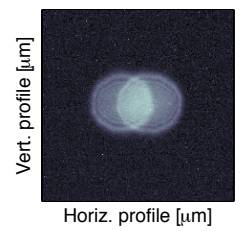

(e)

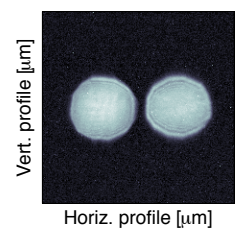

Figure 7.5: NIR beam profiles. The images were taken $2 \mathrm{~mm}(\mathrm{a}), 1 \mathrm{~mm}$ (b) before, at (c), $1 \mathrm{~mm}(\mathrm{~d})$, and $2 \mathrm{~mm}(\mathrm{e})$ after the crystal plane.

\section{Pulse-front tilt alignment}

The pulse-front tilt plays a crucial role in the alignment of the optical setup. Not only the pulse-front tilts of the near-infrared waves have to be paral- 
lel to each other, they also have to be perpendicular to the sum-frequency generated wave. To start with, looking at the SF beam profile gives sufficient information about the orientation of the pulse-front tilts (see Fig. 7.6). If the angle between the fundamental pulse-front tilts is too big, the near field SF beam profile results in a line as can be seen in Fig. 7.6(a). Once

(a)

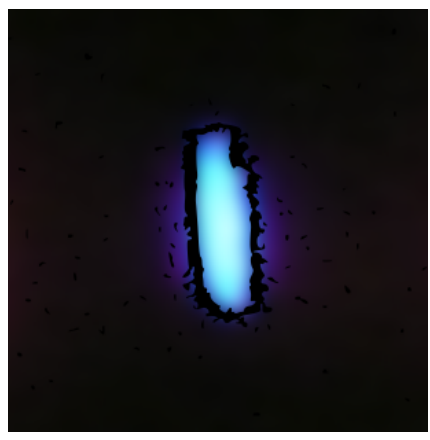

(b)

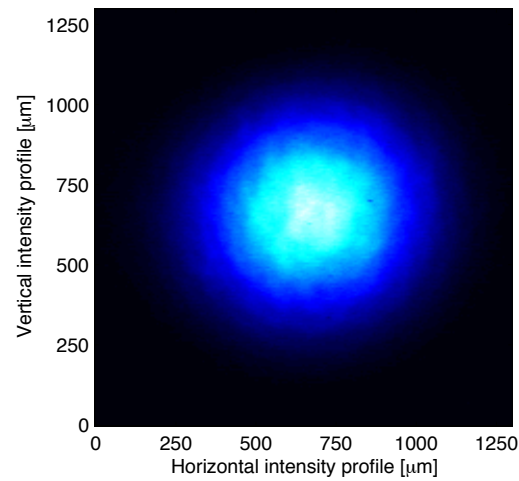

Figure 7.6: SF beam profile with (a) misaligned NIR pulse-front tilts (photo taken of the reflection of a paper) and (b) matched pulse-front tilts (image taken after $1 \mathrm{~m}$ propagation, WinCamD-UCD23).

the NIR pulse fronts are roughly aligned, i.e. the SF beam profile resembles the NIR beam profile (see Fig. 7.6(b)), a detailed alignment can be undertaken. Thereto an iteration between the near field and the far field is necessary. The near field provides information about the parallelism and the far field about the orientation of the pulse front with respect to the SFG vector. The near field was monitored by imaging the crystal plane onto a camera. The left column in Fig. 7.7 illustrates the situation where the pulse fronts cross with an angle and the right column in Fig. 7.7 illustrates the situation where the pulse fronts are parallel. The images were taken for three different pre-delays at intervals of $66.7 \mathrm{fs}$. Depending on the direction of the sweep one can deduce the orientation of the incident pulse-front tilts. Once the fundamental tilts are parallel to each other, it is important to check if the SFG pulse front is perpendicular to his propagation vector, i.e. the sum-frequency generated beam is angular dispersed. This can be easily done in the far field by monitoring the spectrum or by looking at the Fourier plane, i.e. at the beam focus. An angular dispersed beam would be spatially chirped in the far field, the spectrum would vary across the beam. In the Fourier plane the spectral components would be separated 

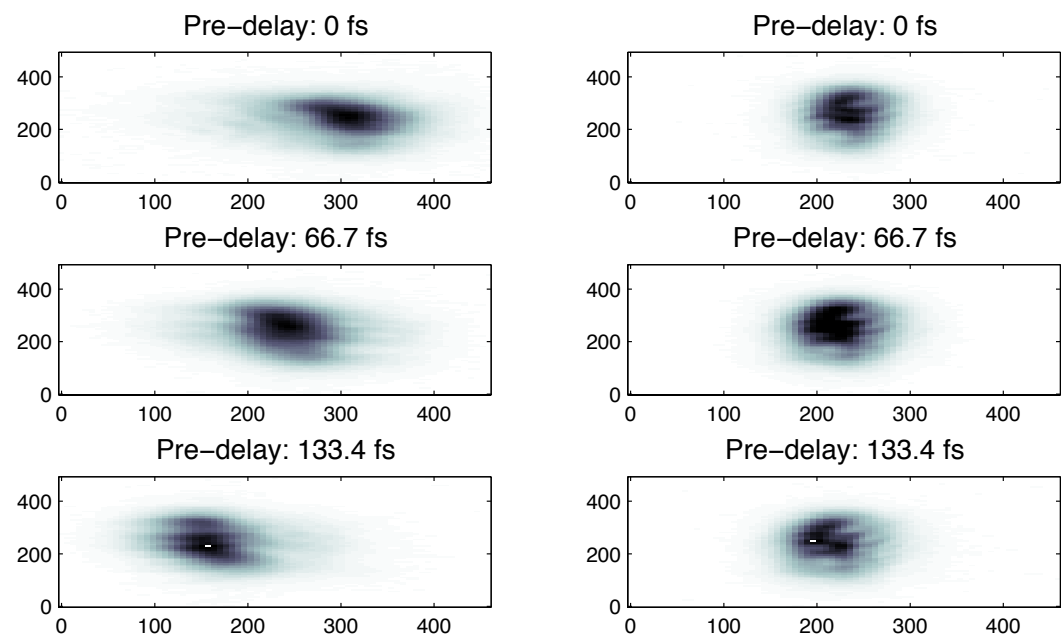

Figure 7.7: Imaged crystal plane. The left column illustrates the situation where the NIR pulse-front tilts are misaligned and the right column the situation where the incident PFTs are parallel. All the axis units (horizontal and vertical position) are in micrometer.

from each other, having minimum beam waists. The focus would appear elongated assuming there is no astigmatism.

\subsection{Results}

Nonlinear pulse compression by sum-frequency generation is demonstrated for sub 100 femtosecond NIR pulses in a type II phasematching $\beta$-Barium Borate crystal. This section deals with the experimental results and demonstrates the potential of this technique to produce ultrashort SF pulses in the ultraviolet with high conversion efficiency.

\subsubsection{Compression of 74 fs long NIR pulses}

By frequency mixing NIR pulses of 74 fs duration using the prism-telescope based setup described in $\S 7.1$, sum frequency pulses as short as 32 fs were obtained. For this experiment the NIR spectrum was cut in the compressor resulting in longer input pulses without applying a linear chirp, i.e maintaining nearly Fourier transform limited pulses. The maximum available pulse energy after the irises, with the aperture diameter set to $2.5 \mathrm{~mm}$, was $200 \mu \mathrm{J}$ for each the ordinary and extraordinary NIR beam. This corresponds to a total intensity of $4 \mathrm{TW} / \mathrm{cm}^{2}$ at the entrance of the BBO crys- 
tal. The SF pulse intensity shapes were measured with a SD-FROG (see $\S 5.2 .2)$ in a $100 \mu \mathrm{m}$ long BBO type I phasematching crystal. The nearinfrared and SF pulse spectra were monitored by a spectrometer (HR 4000 Ocean Optics).

(a)

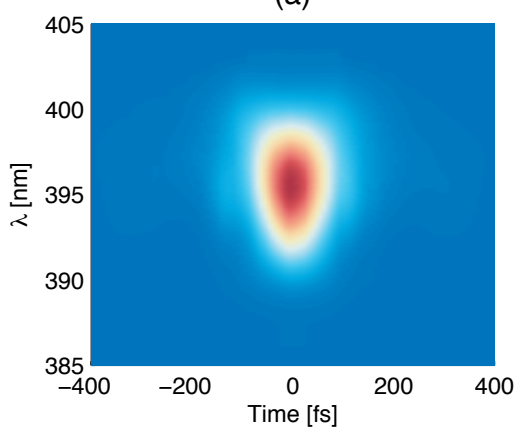

(c)

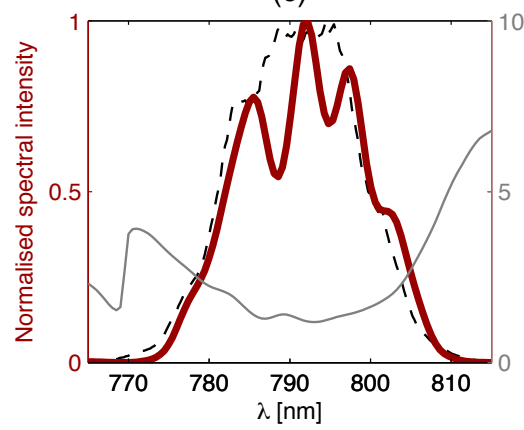

(b)

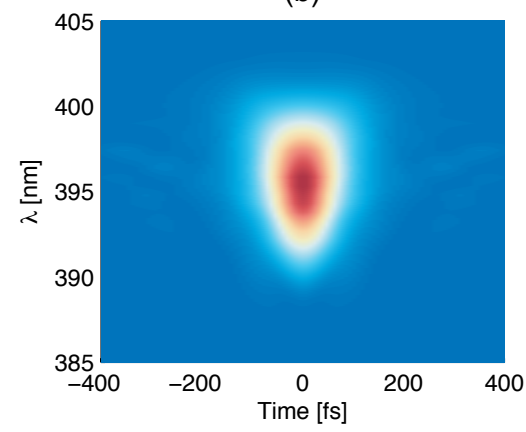

(d)

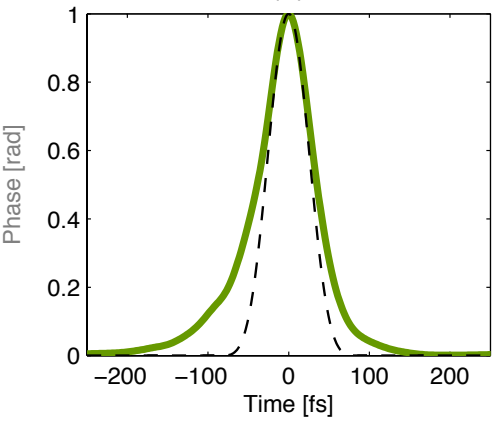

Figure 7.8: Ti:sapphire laser pulse. (a) Measured and (b) reconstructed FROG trace. (c) Retrieved spectrum (solid red line), retrieved spectral phase (solid grey line), and measured spectrum (dashed line). (d) Retrieved temporal intensity (solid green line) and Fourier transform of the measured spectrum (dashed line).

First the incident pulses were measured at the crystal plane. For that the $250 \mu \mathrm{m}$ thick BBO was replaced by a $30 \mu \mathrm{m}$ thin type II phasematching BBO, converting the pulse compression setup into a slightly adapted SHGFROG as described in §5.2.1. The estimated dispersion of $2233 \mathrm{fs}^{2}(6 \mathrm{~mm}$ of F2) induced by the prisms was compensated by changing the grating separation in the compressor of the CPA laser system. The measured and the reconstructed FROG traces are shown in Fig. 7.8(a) and Fig. 7.8(b) respectively. The pulse was retrieved well, with a minimum FROG error ${ }^{2}$ of

\footnotetext{
${ }^{2}$ The root mean square difference between the measured and the computed trace from
} 
0.003 for a $128 \times 128$ pixel trace. The retrieved spectrum (solid red line) with a FWHM of $18 \mathrm{~nm}$ and the measured spectrum (dashed line) are shown in Fig. 7.8(c). The optimized pulse duration (solid green line) is $74 \mathrm{fs}$ at FWHM (see Fig. 7.8(d)) and almost approaches the Fourier transform limit (dashed line) of the measured NIR spectrum in Fig. 7.8(c).

\section{Strong energy exchange}

Once the near-infrared pulse duration is adjusted, it is important to ensure that the pump intensities are high enough, i.e. to find the appropriate energy regime for nonlinear pulse compression. According to the simulation (§4.2.1), it is expected that the SF generated spectra broaden when nonlinear compression takes place. This can be very easily monitored by a spectrometer. Figure 7.9 shows for different pre-delays how the SF spectrum changes as the intensity is increased. At lower intensities, $0.8 \mathrm{TW} / \mathrm{cm}^{2}$, Fig. 7.9(a) resembles strongly a SHG-FROG trace. The only difference is that the trace spans over a longer time range due to the longer crystal length $(250 \mu \mathrm{m}$ instead of $30 \mu \mathrm{m})$ resulting in a longer interaction length. At higher intensities, $4 \mathrm{TW} / \mathrm{cm}^{2}$, spectral broadening is apparent in Fig. 7.9(b), a promising sign for short SFG pulses.

(a)

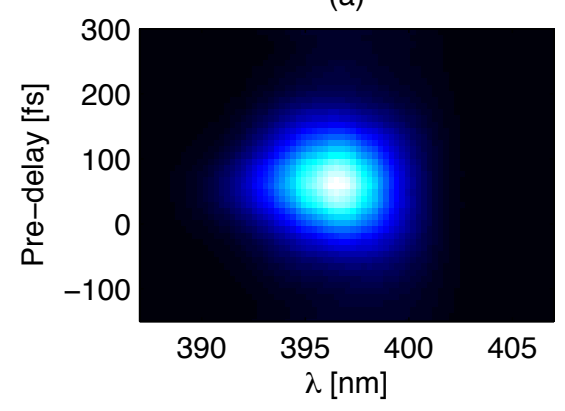

(b)

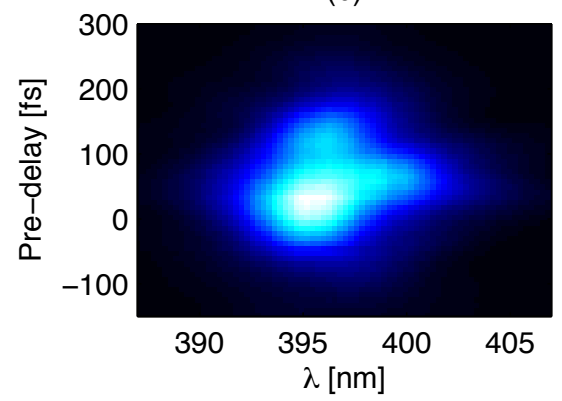

Figure 7.9: SFG spectra as a function of the pre-delay for a total incident intensity of (a) $0.8 \mathrm{TW} / \mathrm{cm}^{2}$ and (b) $4 \mathrm{TW} / \mathrm{cm}^{2}$.

Another good indicator for strong energy exchange is the depletion of the fundamentals which is illustrated in Fig. 7.10. Figure 7.10(a) and (b) show the measured spectra of the extraordinary and the ordinary NIR pump pulses, respectively, as a function of the pre-delay. The plots are in good agreement with the simulated spectra in Fig. 7.10(c) and (d), where the retrieved fundamental pulse displayed in Fig. 7.8(c) was taken as input pulse. 
(a)

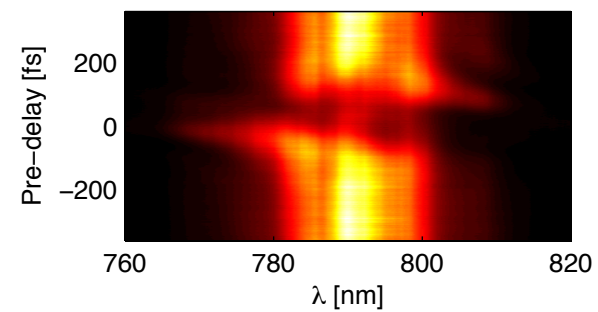

(c)

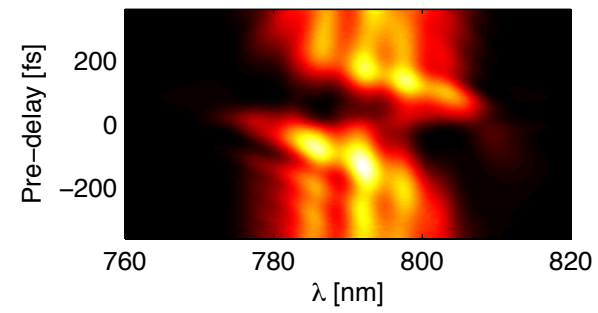

(b)

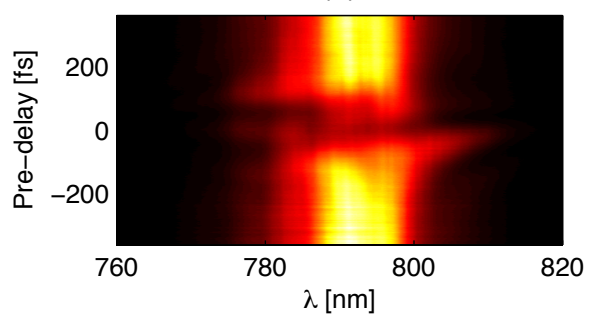

(d)

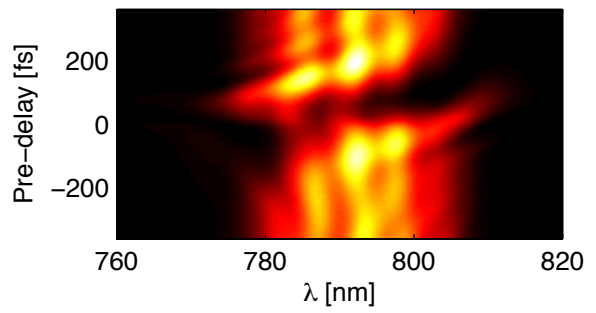

Figure 7.10: Depletion of the NIR fundamentals. Measured spectra of the (a) extraordinary and (b) ordinary polarized pump pulses as a function of the pre-delay. (c) and (d) are the corresponding simulated spectra using the reconstructed pulse shown in Fig. 7.8 as input pulses.

In the nonlinear pulse compression scheme this can be explained by a high energy transfer from the pump pulses to the SF pulses. For appropriate pre-delays ranging in a $100 \mathrm{fs}$ time window together with very high intensities, here $4 \mathrm{TW} / \mathrm{cm}^{2}$ in total, the interaction length is long enough to ensure a high conversion efficiency so that the leading edge of the extraordinary and the trailing edge of the ordinary polarized pulses get depleted. As a consequence of the amplitude modulation there is also a slight spectral broadening visible. Note, that at a specific pre-delay of $50 \mathrm{fs}$, the depletion is most evident and at the same time SF pulse broadening is strongest (see Fig. 7.9(b)).

Relying on computer simulations, pulse compression down to $10 \mathrm{fs}$ and less could be obtained at higher intensities of the NIR pump pulses. In the experiments we were limited by the energy of the pulses produced by the Ti:sapphire laser system at that moment. Increasing the pulse intensity by reducing the beam size before the entrance of the nonlinear pulse compression setup was not acceptable because of: nonlinear effects could arise by passing the beam through the prism; the damage threshold of the optics and the crystal may be approached; and the lateral walk-off in the type II phasematching crystal would become substantial. 


\section{Pulse compression vs delay}

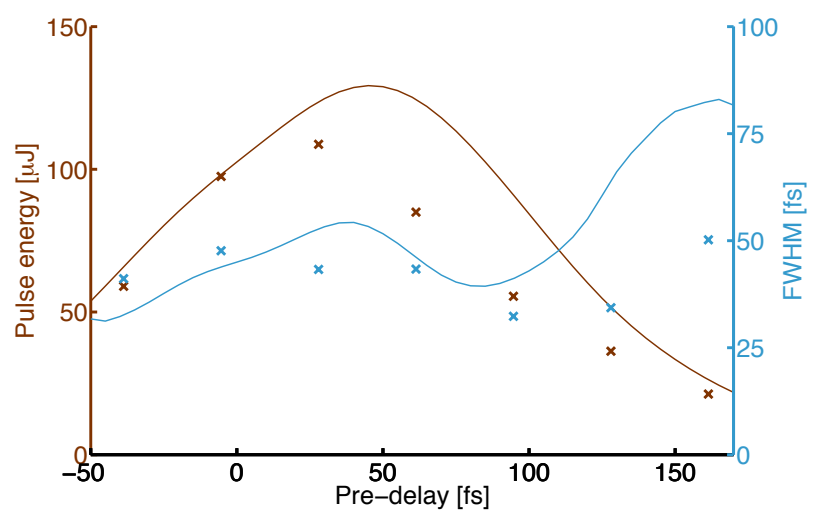

Figure 7.11: SF pulse energy (brown) and pulse width (blue) as a function of the pump pulse pre-delay. Crosses represent experimental data and solid lines represent simulation results.

The retrieved pulse duration at full width at half maximum and the energy of the SF pulse as a function of pre-delay between the pump pulses are reported in Fig. 7.11. Figure 7.12 shows the corresponding measured spectra and retrieved temporal intensities for a restricted range of predelays which is of particular interest. Considerable nonlinear pulse compression was observed for pre-delays ranging between 90-140 fs, i.e. when the o-ray entered the crystal with more than one pulse duration in advance

(a)

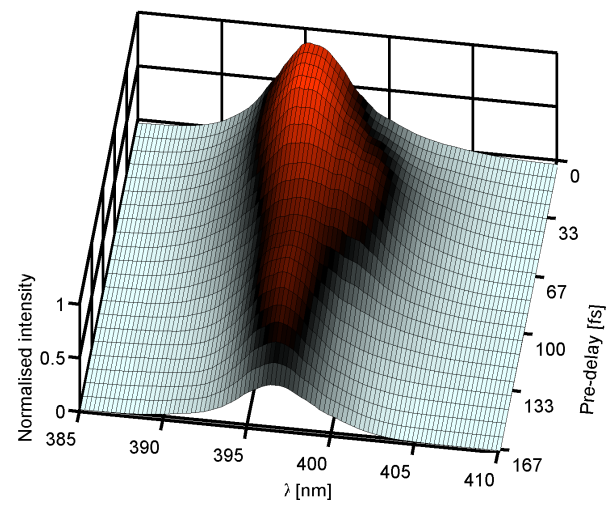

(b)

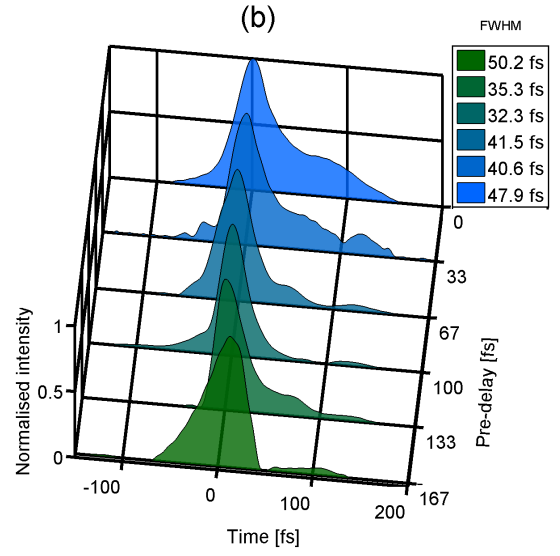

Figure 7.12: (a) Measured sum-frequency generated spectra and (b) reconstructed temporal intensity as a function of the pre-delay. 
of the e-ray. In this situation the e-ray could completely "catch up" to the oray in the crystal while the strong depletion of the NIR provided a very short temporal overlap region over the entire propagation length. For pre-delays ranging between -40 fs and 140 fs the process started with a large to complete superposition of the pump pulses, i.e. a long temporal overlap region. Although the strongest nonlinear interaction between the three waves and substantial spectral broadening, see Fig. 7.12(a), could be observed, the overall pump depletion impeded notable pulse compression. In this range the maximum conversion efficiency of $27 \%$ was achieved for a pre-delay of about $\sim 30 \mathrm{fs}$ between the initial pump pulses. The SF pulse energy was $108 \mu \mathrm{J}$ at this point with a pulse duration of $40 \mathrm{fs}$. Focused down to a $40 \mu \mathrm{m}$ wide spot (100 times the wavelength), this would correspond to an intensity of nearly $2 \cdot 10^{14} \mathrm{~W} / \mathrm{cm}^{2}$, enough to generate high harmonics in gas phase, for example. ${ }^{186}$ For pre-delays below -40 fs and exceeding 40 fs only the tails of the pump pulses overlapped at the start (the e-ray runs away from the o-ray) respectively at the exit of the BBO (the o-ray cannot catch up at the e-ray), which translated in a very weak interaction, i.e. short temporal overlap region.

\section{Results}

We have generated pulses as short as 32 fs by nonlinear pulse compression in a type II phasematching BBO crystal, starting with NIR pulses of

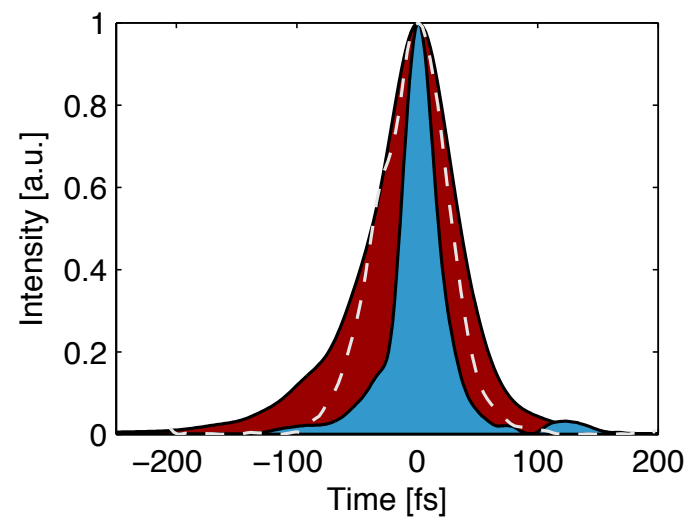

Figure 7.13: Nonlinear pulse compression in the time domain. The fundamental NIR pulse is plotted in red and the compressed SFG generated pulse is plotted in blue. The dashed line shows the SFG signal resulting from a $30 \mu \mathrm{m}$ thick BBO crystal. 
$74 \mathrm{fs}$ duration. The corresponding temporal pulse shapes are overlaid in Fig. 7.13. Also shown (white dashed line) is the SF pulse generated from a $30 \mu \mathrm{m}$ thick BBO crystal with a pulse duration of $67 \mathrm{fs}$ at full width half maximum. The calculated Fourier transform limit of the second harmonic based on the measured NIR spectrum is $42 \mathrm{fs}$. In addition this method allows to generate intense ultrashort pulses of $\sim 45$ fs pulse duration with pulse energies up to $110 \mu \mathrm{J}$. The numerical simulation agrees quite well with the results in Fig. 7.11 and provides a deeper understanding of the physics behind this nonlinear process.

\subsubsection{Compression of sub 50 fs NIR pulses}

Nonlinear compression of ultrashort pulses with durations in the order of

(a)

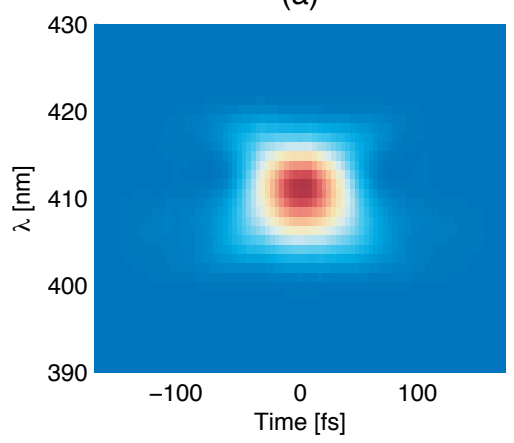

(c)

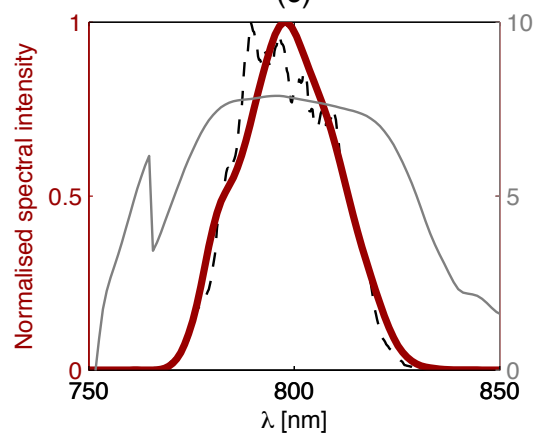

(b)

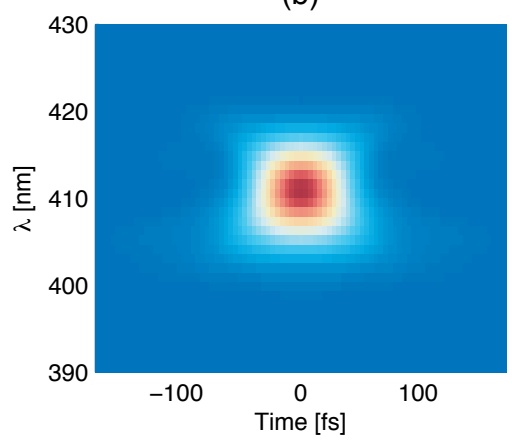

(d)

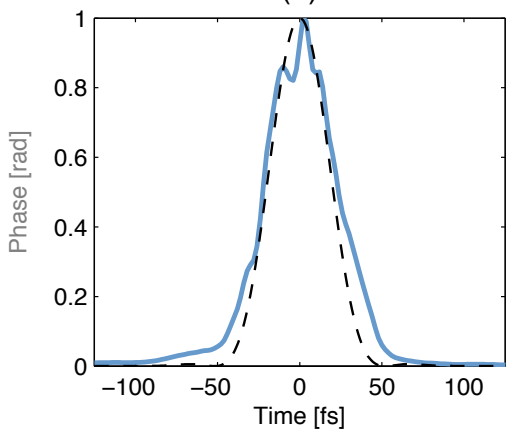

Figure 7.14: Ti:sapphire laser pulse. (a) Measured and (b) reconstructed FROG trace. (c) Retrieved spectrum (solid red line), retrieved spectral phase (solid grey line), and measured spectrum (dashed line). (d) Retrieved temporal intensity (solid blue line) and Fourier transform of the measured spectrum (dashed line). 
30 to $50 \mathrm{fs}$ is a very challenging task as nonlinear parasitic effects become dominant and several thresholds are approached. The reason is that the interaction length decreases with the pulse duration and hence the energy between the interacting pulses must be exchanged faster. As a consequence higher energies are required. Here we present nonlinear SF mixing of 46 fs long NIR pulses in a $250 \mu \mathrm{m}$ type II phasematching BBO crystal.

The incident NIR pulses were characterized using the same SHG-FROG setup as described in §7.2.1. The measured and the reconstructed FROG traces are shown in Fig. 7.14(a) and Fig. 7.14(b). The pulse was retrieved well, with a FROG error of 0.0041 for a $128 \times 128$ pixel trace. The retrieved spectrum (solid red line) with a FWHM of $30.4 \mathrm{~nm}$ is very similar to the measured spectrum (dashed line) (see Fig. 7.14(c). Figure 7.14(d) finally shows the temporal intensity (solid blue line) with a FWHM of 46 fs together with the Fourier transform (dashed line) of the measured NIR spectrum in Fig. 7.14(c). In this experiment the aperture of the irises mounted after the prisms was $2 \mathrm{~mm}$. The maximum energy after the irises was $170 \mu \mathrm{J}$ per pulse corresponding to an intensity of $\sim 3.5 \mathrm{TW} / \mathrm{cm}^{2}$ at the crystal plane, i.e. after the downsizing telescope which reduces the beam by a factor 6 . The calibrated intensity distribution is illustrated in Fig. 7.4. We measured the SFG pulses for various input energies starting from $57.5 \mu \mathrm{J}$ to $170 \mu \mathrm{J}$ per NIR pulse. The energy range was chosen in a way to cover sumfrequency generation at low energies (still sufficient to perform SD-FROG measurements) until very high energies near the damage threshold of $\beta$ Barium Borate. Hereby it should be possible to characterize the process of sum-frequency generation in the presence of group-velocity mismatch from modest nonlinear interactions to nonlinear pulse compression. Figure 7.15 illustrates the SFG spectra as a function of the pre-delay between the fun-

(a)

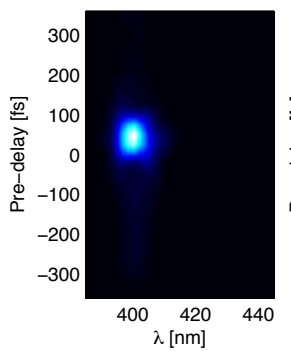

(b)

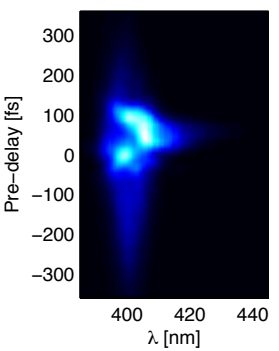

(c)

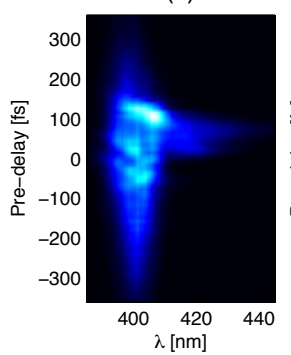

(d)

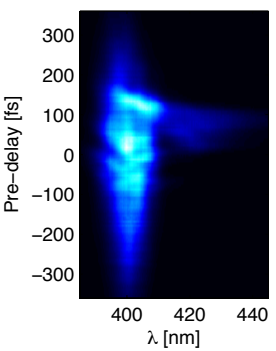

Figure 7.15: SFG spectra as a function of the pre-delay at a total incident intensity of (a) $1 \mathrm{TW} / \mathrm{cm}^{2}$, (b) $3 \mathrm{TW} / \mathrm{cm}^{2}$, (c) $5 \mathrm{TW} / \mathrm{cm}^{2}$, and (d) $7 \mathrm{TW} / \mathrm{cm}^{2}$ 
damentals for input energies starting at $57.5 \mu \mathrm{J}$ to $170 \mu \mathrm{J}$ per pump pulse. At low energies we can observe simple type II second-harmonic generation and modest energy transfer from the NIR waves to the SF wave. For input energies around $80 \mu \mathrm{J}$ per pulse most of the spectral energy is shifted to longer wavelengths and for energies bigger than $100 \mu \mathrm{J}$ strong spectral broadening towards the NIR can be observed for pre-delays between 30 fs and $100 \mathrm{fs}$. One can also see that the temporal overlap region increases with energy.

(a)

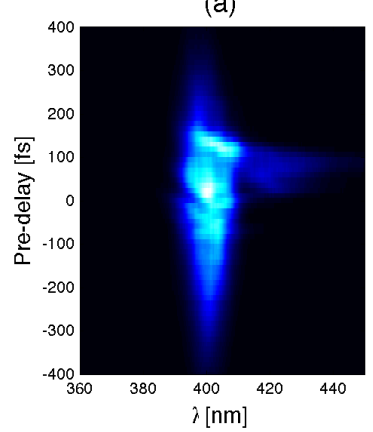

(b)

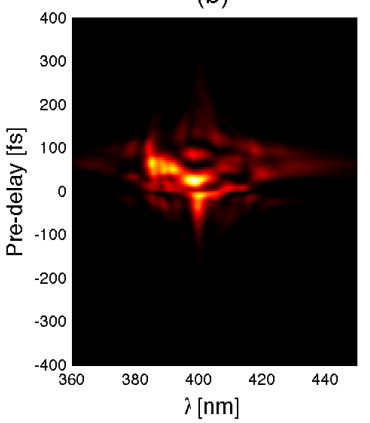

(c)

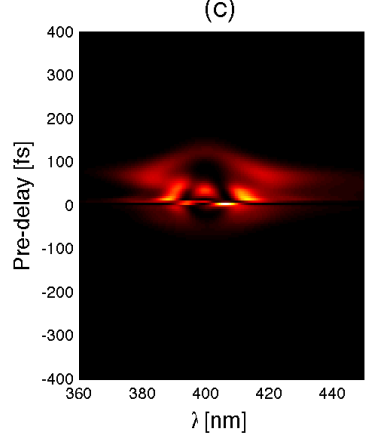

Figure 7.16: SFG spectra as a function of the pre-delay for a total incident intensity of $8 \mathrm{TW} / \mathrm{cm}^{2}$. (a) Measured spectra, (b) simulation using reconstructed pulses, and (c) simulation using Gaussian input pulses.

The measured and simulated sum-frequency generated spectra as a function of the pre-delay are compared in Figure 7.16. Figure 7.16(a) shows the measured SFG spectra, Fig. 7.16(b) the simulated spectra using the retrieved pulse of Fig. 7.14(d) as input pulse, and Fig. 7.16(c) the simulated spectra using a Gaussian pulse with a duration of 46 fs at FWHM. The sum-frequency generation should be insignificant for pre-delays bigger than $200 \mathrm{fs}$ and smaller than $-50 \mathrm{fs}$, since no overlap of the fundamentals is expected. A most straightforward explanation would be that the pulse-front tilts of the pump pulses are not parallel to each other, but a misalignment would be immediately visible at very low energies. This is not the case as can be seen in Fig. 7.15(a). Another different feature is the spectral broadening towards the UV which appears in the simulation and which is not present in the measured data. The specific double peak structure is clearly visible in the simulation using Gaussian input pulses for pre-delays ranging from 50 fs to 110 fs and is apparent for a much smaller pre-delay range (50 fs to $70 \mathrm{fs}$ ) using the retrieved pulses. This feature could not be observed in the laboratory.

In Fig. 7.17 we compare the reconstructed sum-frequency pulses for 
(a)

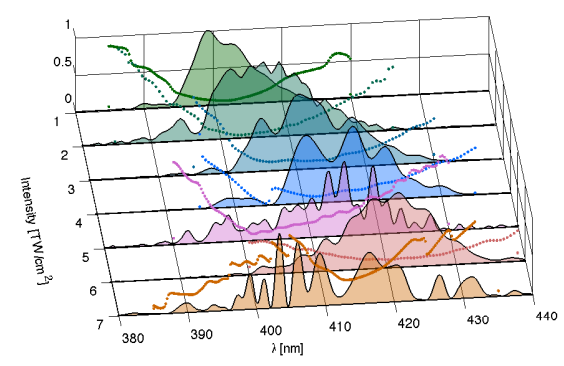

(b)

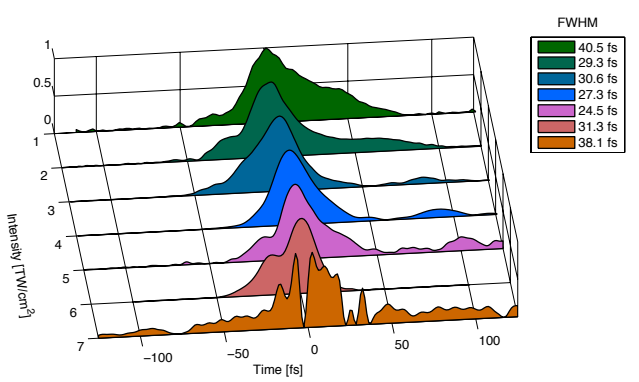

Figure 7.17: Reconstructed SFG pulse intensities in the (a) frequency domain (phase - dotted lines - is in radians divided by a factor 10) and in the (b) time domain for various incident (total) intensities for a pre-delay of $50 \mathrm{fs}$.

total incident energies ranging from $1 \mathrm{TW} / \mathrm{cm}^{2}$ to $7 \mathrm{TW} / \mathrm{cm}^{2}$. The delay between the fundamentals was fixed at $50 \mathrm{fs}$ and was chosen for maximum energy conversion. We can see that at lower input energies the pulses are longer and that the tails get shorter and shorter. At highest intensities we observe pulse splitting, probably as a consequence of strong backconversion.

\section{Results}

We have generated pulses as short as $25 \mathrm{fs}$ in the UV by nonlinear pulse compression in a type II phasematching BBO crystal, starting with nearinfrared pulses of $46 \mathrm{fs}$ duration. SF pulse energies as high as $31 \mu \mathrm{J}$ could be achieved by mixing two NIR pulses with a total energy of $270 \mu \mathrm{J}$. The retrieved pulse durations at full width half maximum of the SF pulses as a function of the pre-delay between the pump pulses are reported in Fig. 7.18 (green dots) for a fixed total intensity of $4 \mathrm{TW} / \mathrm{cm}^{2}$, i.e. where the shortest pulses were observed. The numerical results, using the reconstructed near-infrared spectra of Fig. 7.14(c) (solid pink line) and Gaussian pulses with a FWHM of 46 fs (solid grey line) as input, qualitatively reproduce some trends. For pre-delays ranging between -25 and 25 fs (approximately half the duration of a fundamental frequency pulse), no pulse compression was observed. For increasing pre-delays the SF pulse durations decrease and for pre-delays bigger than $\sim 100$ fs they start increasing again. Nevertheless sum-frequency generation of sub-10 fs pulses could 
not be observed experimentally.

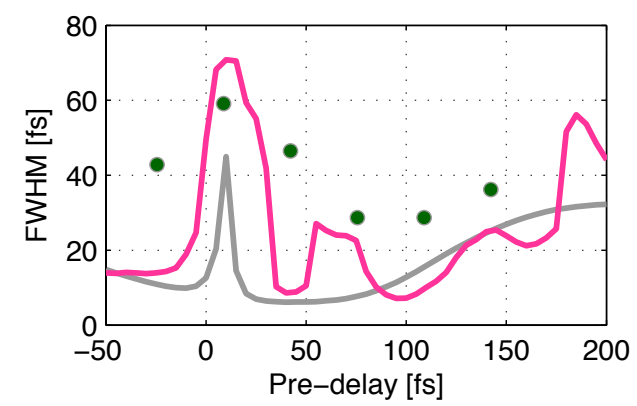

Figure 7.18: SF pulse durations at FWHM as a function of the predelay. Simulated data with Gaussian (solid gray line) and reconstructed NIR pulses (solid pink line) as input and measured data (green dots). The total incident intensity was $4 \mathrm{TW} / \mathrm{cm}^{2}$ in all three cases.

\subsubsection{Discussion}

The results detailed in this section demonstrate nonlinear pulse compression via sum-frequency generation of femtosecond NIR pulses at very high intensities in a type II BBO crystal using a noncollinear geometry. Good agreement between the theoretical and experimental curves is found. As with any technique, there are certain limitations and an opportunity for improvement.

For instance unwanted nonlinear effects caused by very high intensities, especially for pulse durations below $50 \mathrm{fs}$, seem to reduce the efficiency of the pulse compression. This becomes very clear in Fig. 7.19

(a)

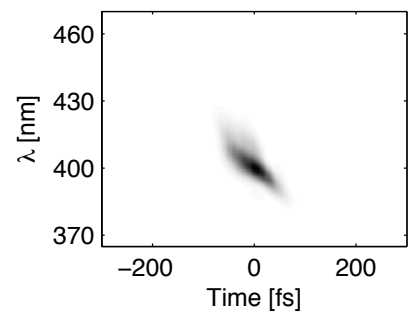

(b)

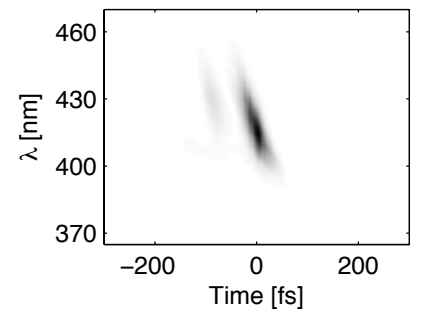

(c)

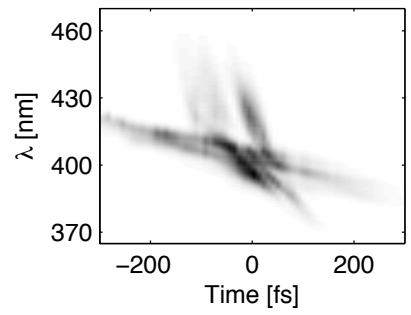

Figure 7.19: Measured SD-FROG traces for a total incident intensity of (a) $1 \mathrm{TW} / \mathrm{cm}^{2}$, (b) $4 \mathrm{TW} / \mathrm{cm}^{2}$, and (c) $7 \mathrm{TW} / \mathrm{cm}^{2}$. The pre-delay between the fundamentals is 50 fs for all traces. 
where the measured SD-FROG traces are plotted for increasing intensities. The SF pulses are getting more and more complex and the noise is increasing. Probably most of these features can be attributed to nonlinear refractive index effects and to nonlinear thermal effects.

The propagation of an intense pulse will induce changes in the index of refraction in the traversed medium leading possibly to self-phase modulation and self-focusing. While self-phase modulation originates from the time-dependent change in the refractive index induced by a laser pulse with an intensity envelope $I(t)$ varying in time, self-focusing is related to a nonlinear lens induced by a laser beam with a spatially non-uniform intensity distribution $I(r)$. Self-phase modulation is the change in the phase of an optical pulse resulting from the nonlinearity of the refractive index of the medium. For a sample with a thickness $L$, the phase of the transmitted pulse changes by the amount

$$
\phi_{N L}(t)=-n_{2} I(t) \omega_{0} L / c,
$$

and the maximum value of the frequency shift is of the order

$$
\Delta \phi_{N L}^{(\max )} \backsim n_{2} \frac{\omega_{0}}{c} I_{0} L,
$$

where $\omega_{0}$ is the centre frequency of the pulse, $n_{2}$ is the nonlinear refractive index of the material, and $c$ is the speed of light. As a result of the time-varying phase of the wave, the spectrum of the transmitted pulse will be modified and typically will be broader than that of the incident pulse (see also Fig. 4.9 in Chap. 4). We expect that spectral broadening due to self-phase modulation will be very important whenever $\Delta \phi_{N L}^{(\max )} \geq 2 \pi$. For example a pulse centred at $400 \mathrm{~nm}$ with an energy of $30 \mu \mathrm{J}$ and a beam diameter of $333 \mu \mathrm{m}$ would result in a frequency shift of $\sim 0.85 \pi$. Being caused by the same change in index, self-focusing affects the beam profile, unless the intensity of the input beam does not vary transversely to the propagation direction, i.e the beam profile is "flat". Considering a Gaussian beam with a beam waist $w_{0}$, the characteristic self-focusing distance calculates as

$$
z_{s f}=w_{0} \sqrt{\frac{n_{0}}{2 n_{2} I}},
$$

where $n_{0}$ is the linear refractive index. The nonlinear lens can become so strong that the beam collapses to a very small radius, resulting in strongly increased optical intensities which can lead to damage of the material or to a beam breakup into many filaments. 
Thermal processes can also lead to large, usually unwanted, nonlinear effects. The origin of thermal nonlinear effects is that the conversion efficiency never approaches $100 \%$. In BBO for example, local heating can arise from the linear absorption of blue (second harmonic) and ultraviolet (fourth harmonic) radiation. The local increase of temperature induces changes in index of refraction. We can distinguish two main thermal effects caused by non-uniform heating, thermal lensing and thermal dephasing. While thermal dephasing is a longitudinal thermal effect, thermal lensing is an in-plane thermal effect. Thermal dephasing refers to the change of the phasematching angle, determined by the temperature dependent refractive indices, as the crystal is heated. The thermal lens arises from the transverse gradient of the refractive index caused by the non-uniform temperature distribution. Further index changes can be caused by thermally induced mechanical stress which can also lead to bulging of the end faces, in particular of very thin crystals. The thermal lens power $p_{t h}$, the inverse of the focal length $f_{t h}$, can be estimated with the following equation,

$$
p_{t h}=\frac{1}{f_{t h}}=\frac{P^{a b s}}{\pi w_{0}^{2}} \frac{d n / d T}{\kappa},
$$

where $P^{a b s}$ is the total absorbed power, $w_{0}^{2}$ is the radius of the pump beam, $d n / d T$ is the thermooptic coefficient, and $\kappa$ is the thermal conductivity. The induced thermal lens can be focusing or diverging depending on the medium. In the case of BBO $d n / d T$ is negative ${ }^{157}\left(d n_{o} / d T=\right.$ $-16.6 \times 10^{-6} / \mathrm{K}$ and $d n_{e} / d T=-9.3 \times 10^{-6} / \mathrm{K}$ ) and thus the induced lens will be diverging. In short, thermal effects in the material will limit the conversion efficiency due to self-induced phase mismatch through the crystal and reduce the beam quality.

(a)

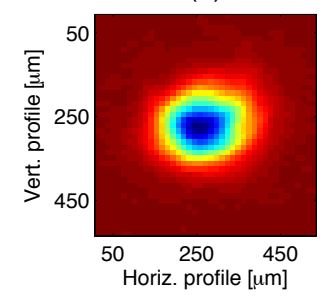

(b)

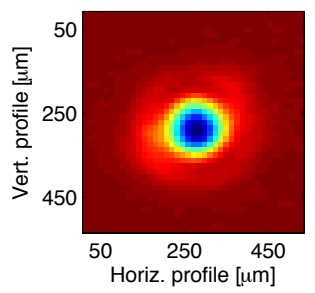

(c)

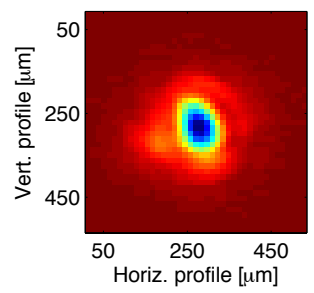

(d)

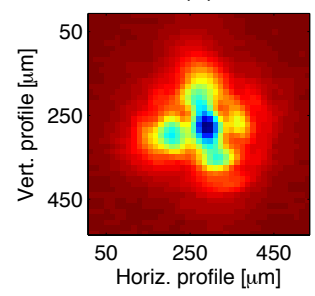

Figure 7.20: Influence of a non-uniform pump profile. SF beam profile after one meter for a total incident intensity of (a) $0.6 \mathrm{TW} / \mathrm{cm}^{2}$, (b) $3 \mathrm{TW} / \mathrm{cm}^{2}$, (c) $6 \mathrm{TW} / \mathrm{cm}^{2}$, and (d) $8.5 \mathrm{TW} / \mathrm{cm}^{2}$.

Figure 7.20 illustrates the importance of a flat beam profile. It shows the effect of a non-uniform pump beam profile, i.e. no iris is introduced 
in the beam path, on the SF beam profile after one meter of propagation. With increasing power the SF beam profile gets smaller and smaller. This indicates that the Kerr effect, responsible for self-focusing, is stronger than the thermal effect, inducing a diverging lens. It would be hence beneficial to implement an optical system which converts a Gaussian to a flattop beam profile instead of using an iris.

Although great care was taken, it is very difficult to ensure that the SF pulse has no pulse-front tilt at all, since not only the PFTs of the NIR fundamentals have to be parallel to each other but at the same time perpendicular to the wavevector of the SF wave. Furthermore it is possible that the phasematching angles change with increasing intensities due to Kerr and thermal nonlinearities. A perfect alignment at low intensities can be slightly misaligned at high intensities. The consequences are dramatic as shown in Fig. 7.21. Already a small pulse-front tilt of $0.2^{\circ}$ would cause a pulse with a duration of $10 \mathrm{fs}$ and a centre wavelength of $400 \mathrm{~nm}$ to broaden to $25 \mathrm{fs}$ after one meter of propagation.

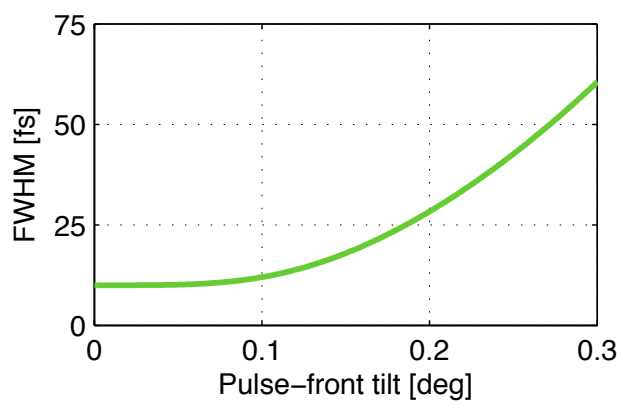

Figure 7.21: Temporal pulse broadening after $1 \mathrm{~m}$ as a function of the pulse-front tilt. The initial pulse has a pulse duration at full width half maximum of $10 \mathrm{fs}$ and its centre wavelength is $400 \mathrm{~nm}$.

It should be stressed that a single-shot diagnostic is highly desirable in the context of alignment. A single-shot FROG instead of a scanning FROG would have the advantage that changes in the experimental parameters could be observed in real time. Note that such measurement device is not suitable for energies below $\sim 10 \mu \mathrm{J}{ }^{187}$

\subsection{Conclusion}

In this chapter I have presented a very compact setup based on transmission gratings, suitable for the compression of picosecond pulses, and 
a more complex setup based on prism-telescopes, suitable for the compression of femtosecond pulses. Compression of near-infrared pulses with a duration of $74 \mathrm{fs}$ down to $32 \mathrm{fs}$ as well as compression of 46 fs pulses down to 25 fs could be demonstrated. The measurements are in good agreement with the simulations carried out in Chap. 4 and demonstrate the validity of the code for the investigation of noncollinear sum-frequency generation. The results indicate that third-order nonlinear processes and nonlinear thermal effects may limit the conversion efficiency of the pulse compression technique for sub 50 fs pulses. A non-uniform beam profile may lead to a blurring of the shortest pulses and self-focusing of the sumfrequency generated beam. Finally not only the spatial beam profile of the pump pulses is crucial for efficient compression but also the temporal shape. A smooth temporal intensity distribution with steep tails is required to avoid reconversion of the SF wave to the fundamental waves resulting in energy reduction, distortion, and in the worst case splitting of the SF pulses. 


\section{Chapter 8}

\section{Summary and outlook}

The work presented in this thesis (introduced in chapter one) is focused on the generation of intense femtosecond pulses in the ultraviolet and near- to mid-infrared region.

In the second chapter the most important optical pulse compression techniques are introduced based on spectral broadening via self-phase modulation followed by a negative dispersive delay line. The state of the art laser sources delivering intense few-cycle pulses in the NIR region is given. Nonlinear pulse compression is presented as an alternative method with the advantage of being scalable in energy and being capable of compressing bandwidth-limited pulses in a single step. The existing laser sources delivering ultrashort pulses in the UV and in the NIR to MIR spectral region together with their applications are described afterwards.

The third chapter is devoted to the theory of nonlinear three-wave interaction. Based on Maxwells equations, the coupled-wave equations for sum-frequency generation are derived assuming plane waves and taking into account group-velocity mismatch and group-velocity dispersion effects. It is also shown that the combination of pulse-front tilt and crystal birefringence permits exact phasematching in the context of group-velocity mismatch using a noncollinear geometry. The noncollinear angles, the angular dispersion, the group velocities, and the pulse-front tilts are calculated in dependence of the crystal cut angle in a type II $\beta$-Barium Borate crystal.

A Matlab code simulating the sum-frequency process is developed in chapter four in order to study the evolution of the three interacting waves inside a nonlinear medium. The numerical results are then presented pointing out the possible influence of some relevant experimental parameters. This way the effects of the fundamental pulse durations and intensities, the initial pre-delay between the pump pulses, and the crystal cut angle on the 
sum-frequency generated laser pulse can be clarified. The main result is that under ideal conditions, i.e. perfect Gaussian input pulses, pulse compression factors greater than six can be achieved, e.g. starting with NIR pulses of 40 fs pulse duration and generating UV pulses of $\sim 6$ fs pulse duration.

The first part of chapter five describes the operating principle of an amplifier laser system based on chirped pulse amplification. The oscillator, the stretcher, the amplifier, and the grating compressor are explained in detail. The second part of chapter five deals with the diagnostic of femtosecond pulses: SHG-FROG, SPIDER and PHAZZLER for the full amplitude and phase characterization of near-infrared pulses and SD-FROG for ultraviolet pulses.

Chapter six deals with the generation of ultrashort pulses through optical parametric amplification in a gas-filled hollow-core fibre. By combining the output of a Ti:sapphire amplifier and its second harmonic in argon, near-infrared pulses with sub 50 fs duration are reported.

Chapter seven is dedicated to the nonlinear pulse compression experiment. Two noncollinear setups are described, one using transmission gratings and the other one using a combination of a prism and a downsizing telescope to induce the desired pulse-front tilt. Finally the main experimental results using ultrashort amplified Ti:sapphire laser pulses are presented; nonlinear pulse compression of NIR pulses with a duration of 74 fs (and 46 fs) down to $32 \mathrm{fs}$ (respectively $25 \mathrm{fs}$ ) has been observed. This section is followed by a discussion about the opportunities for improvement and the limitations concerning this technique.

To conclude, we have studied two nonlinear processes for generating ultrashort laser pulses in spectral regions which are ordinarily difficult to achieve by the existing techniques. The first involves optical parametric amplification mediated by four-wave-mixing in gas and supports the generation of ultrashort pulses in the NIR to MIR spectral region. The four-wavemixing process involved in the OPA is expected to lead carrier-envelope phase stable pulses which is of great importance for applications in extreme nonlinear optics. These NIR to MIR pulses can be used directly for nonlinear light-matter interactions making use of its long-wavelength characteristics. We also demonstrate efficient nonlinear pulse compression by sum-frequency mixing ultrashort pulses from a Ti:sapphire amplifier laser in appropriate conditions of group-velocity mismatch. Proposed by Zhang ${ }^{66}$ in 1990, a noncollinear geometry was implemented for the first time in the context of nonlinear pulse compression. This is of high interest, as this method allows compression for a variety of new wavelength regimes which 
until now were out of reach because of the lack of suitable crystals. In a noncollinear interaction there is an additional degree of freedom, the noncollinear angles of the fundamentals. By suitably selecting these angles, it is often possible to achieve simultaneously phasematching under the required GVM conditions. An important advantage of the presented scheme, compared to a collinear three-wave interaction with tilted input pulses, ${ }^{188}$ is that the required energy front tilts are approximately three times smaller and that the SF generated pulses emerge untilted. Adjusting the group velocities in noncollinear interactions has the obvious drawback of limiting the effective-interaction volumes to the region of geometrical overlap of the pump beams. This is no problem for femtosecond pulses as the interaction length is in the order of 200 to $400 \mu \mathrm{m}$ but cannot be neglected for picosecond pulses. Compared to state-of-the-art compression techniques based on self-phase modulation, pulse compression by sum-frequency generation is free of aperture limitation, and thus scalable in energy. Nevertheless, we point out that $\chi^{(3)}$ processes and nonlinear thermal effects in the crystal are the main limiting factors for nonlinear pulse compression in the sub-50 fs temporal domain where intensities greater than $1 \mathrm{TW} / \mathrm{cm}^{2}$ are needed. Note that care has to be taken regarding the damage threshold when other crystals than BBO are used. Summing up, noncollinear nonlinear pulse compression (NNPC) could extend significantly the field of applications in extreme nonlinear optics, e.g., by studying ultrafast dynamics of a variety of (bio)molecular systems.

\section{Outlook}

The results of the work described open a number of possibilities for future work besides the improvement of the experimental setup like a single-shot diagnostic or the generation of flat-top pump beams. I want to draw the attention on two promising options: energy scaling and wavelength diversity.

The Ti:sapphire laser system, developed as part of the work, is currently delivering energies ten times higher than the ones used for the presented experiments. With some minor changes, e.g., replacement of some optics by bigger ones, it should be straightforward to apply the NNPC technique to the compression of pulses of even higher energy. Pulse compression by sum-frequency generation is free of aperture limitation compared to other compression techniques where $\chi^{(3)}$ effects in the form of self-phase modulation are part of the compression scheme. The same $\chi^{(3)}$ effects in the form of self-focusing are responsible for beam breakdown, thus imposing an energy limit. With our laser system It should then be possible 
to generate ultrashort UV pulses with energies as high as $300 \mu \mathrm{J}$. These pulses will allow to generate high harmonics with much higher efficiency than ultrashort pulses in the near-infrared with similar energies.

As mentioned before, the proposed compression technique could be adapted to a variety of birefringent crystals with the aim of generating ultrashort pulses at different wavelengths. For example, the output of a Ti:sapphire laser $(800 \mathrm{~nm})$ could be combined with either the signal (1200 $\mathrm{nm})$ or the idler $(2000 \mathrm{~nm})$ pulses of a traveling wave parametric amplifier (e.g., TOPAS, Light Conversion) in a type II BBO, thus generating ultrashort pulses at $480 \mathrm{~nm}$ or at $580 \mathrm{~nm}$ respectively. The internal noncollinear angles between the pump pulses would lie around $20^{\circ}$. Note that this technique would allow to obtain tunable ultrashort pulses if combined with a TOPAS where one of the two pump pulses is frequency tunable and the variation of its group velocity is small in the tuning range. ${ }^{63}$ Another interesting option is to mix the output of a Ti:sapphire laser with its second harmonic in a BBO crystal (total noncollinear angle $\sim 30^{\circ}$ ). This way intense UV pulses centred at $266 \mathrm{~nm}$ could be generated. Such femtosecond pulses in the visible and in the ultraviolet are very desirable for studying ultrafast dynamics of a variety of molecular systems. 


\section{Appendix A}

\section{Transmission grating damage report}

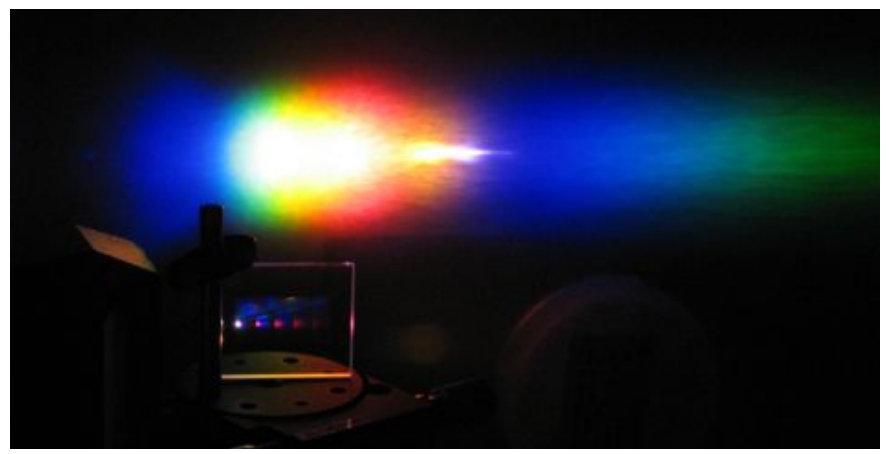

\section{Introduction}

The threshold fluence for laser induced damage on a transmission grating was determined. The grating of interest is a transmission grating designed for pulse compression operating at a center wavelength of $1064 \mathrm{~nm}$ with a grating resolution of $1250 \mathrm{l} / \mathrm{mm}$ made out of UV grade fused silica from Ibsen Photonics (FSTG-PCG-1250-1064). Measurements were conducted using the output of an amplified Ti:Sapphire laser system delivering pulses at $40 \mathrm{fs}$ at a repetition rate of $3 \mathrm{kHz}$ centred at $785 \mathrm{~nm}$. The damage was then determined with a phase contrast microscope. 


\section{Procedure to determine the damage threshold}

Figure A.1 shows the beam profile at the equivalent grating plane. The effective area assuming a Gaussian beam waist is $2.245 \times 10^{4} \mu \mathrm{m}^{2}$. The

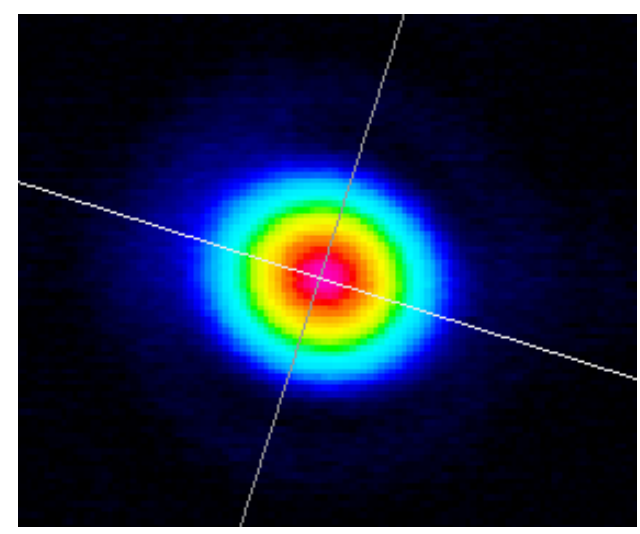

Figure A.1: Beam profile at the grating plane.

grating was exposed to different pulse fluences varying from $0.28 \mathrm{~J} / \mathrm{cm}^{2}$ to $1.22 \mathrm{~J} / \mathrm{cm}^{2}$. For that the pulses from a Ti:Sapphire laser system were sent through a half-wave plate mounted on a rotation mount followed by a polarizing beam splitter to adjust the different energies. The beam was then focused with a lens $(f=1 \mathrm{~m})$ and sent onto the grating (see Fig. A.2). The grating was mounted on a $x-y$ translation stage which was moved in a raster pattern for the different energies. Two measurement runs were performed applying the following fluences: $1.0572 \mathrm{~J} / \mathrm{cm}^{2}, 1.2176 \mathrm{~J} / \mathrm{cm}^{2}$, $1.1433 \mathrm{~J} / \mathrm{cm}^{2}, 0.9800 \mathrm{~J} / \mathrm{cm}^{2}, 0.8018 \mathrm{~J} / \mathrm{cm}^{2}, 0.6682 \mathrm{~J} / \mathrm{cm}^{2}, 0.4826 \mathrm{~J} / \mathrm{cm}^{2}$, $0.3861 \mathrm{~J} / \mathrm{cm}^{2}, 0.2851 \mathrm{~J} / \mathrm{cm}^{2}$ for the first run and $1.1879 \mathrm{~J} / \mathrm{cm}^{2}, 1.1879$ $\mathrm{J} / \mathrm{cm}^{2}, 1.1136 \mathrm{~J} / \mathrm{cm}^{2}, 1.0394 \mathrm{~J} / \mathrm{cm}^{2}, 0.9800 \mathrm{~J} / \mathrm{cm}^{2}, 0.8909 \mathrm{~J} / \mathrm{cm}^{2}, 0.7424$ $\mathrm{J} / \mathrm{cm}^{2}, 0.5791 \mathrm{~J} / \mathrm{cm}^{2}$ for the second run. The exposure time for every tested spot was $5 \mathrm{~s}$. A reflection of the spectrum and the first order of the grating were monitored by a spectrometer (HR 4000, Ocean Optics).

In a second step the easily visible damages were identified with a microscope having a magnification of 10 . To determine the physical damage for the different fluence levels around the damage threshold, a phase contrast measurement (magnification 40) was performed. 


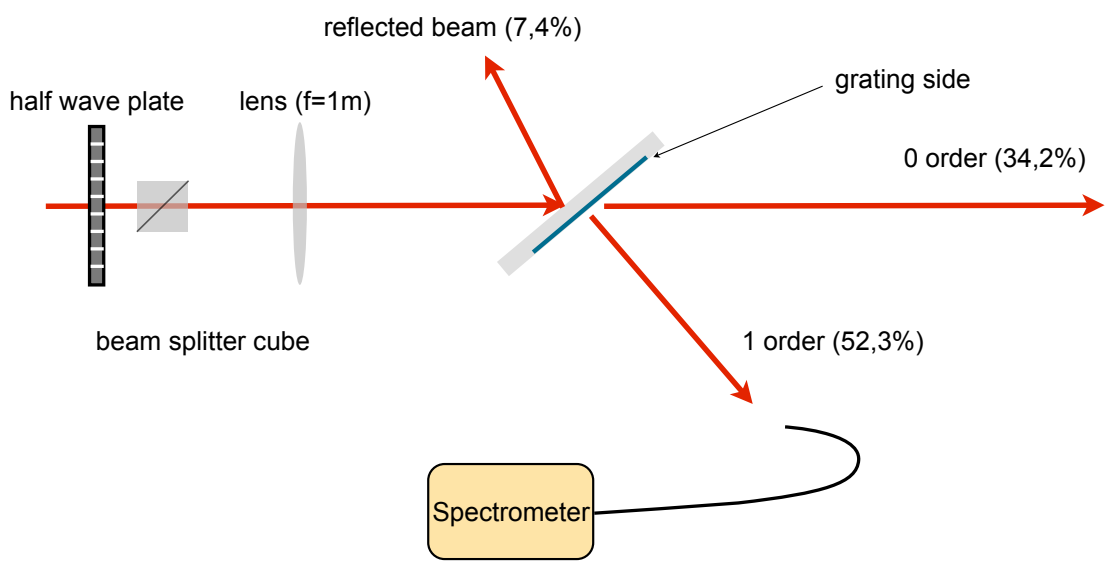

Figure A.2: Experimental setup for testing the damage threshold of the grating. The percentages indicate the amount of energy in the diffracted and reflected beams compared to the incident beam.

\section{Results and discussion}

The goal of this test was to determine at which fluences physical damage on a transmission pulse compression grating appears. Self-phase modulation was already detected at much lower intensities, but was not part of this investigation.

For all tested fluences no damage to the actual grating lines could be identified. The damage always occurred on the UV-grade fused silica side which agrees with the self-collapse distance estimated with a simple approximation. ${ }^{189}$ The latter lies between $1.1 \mathrm{~mm}$ and $2.2 \mathrm{~mm}$ for input intensities of $30.4 \mathrm{TW} / \mathrm{cm}^{2}$ and $7.1 \mathrm{TW} / \mathrm{cm}^{2}$ respectively. Figure A.3 shows the microscope images of the fused silica side of the grating with the visible damage for fluences above $0.9800 \mathrm{~J} / \mathrm{cm}^{2}$ or $23 \mathrm{TW} / \mathrm{cm}^{2}$.

The lowest fluence inducing damage on the grating was registered at $1.0394 \mathrm{~J} / \mathrm{cm}^{2}$, corresponding to a peak intensity of $24.4 \mathrm{TW} / \mathrm{cm}^{2}$, which is consistent with the measurements on dielectrics taken by Lenzner et al. ${ }^{190}$ For fluences between $1.0439 \mathrm{~J} / \mathrm{cm}^{2}$ and $1.1433 \mathrm{~J} / \mathrm{cm}^{2}$, the damage mostly appeared as a small approximately 4 micron diameter spot under the phase contrast microscope, but could not be detected using the normal microscope with a magnification of 10 . Above $1.1433 \mathrm{~J} / \mathrm{cm}^{2}$, the damage appeared as a collection of spots with an overall diameter of approximately 100 micron. 


\begin{tabular}{|c|c|c|}
\hline Fluence & Microscope & Comment \\
\hline $1.22 \mathrm{~J} / \mathrm{cm}^{2}$ & & $\begin{array}{l}\text { Backscatter, white spot } \\
\text { visible by eye and SPM. } \\
\text { Magnification } 40 x \text {. }\end{array}$ \\
\hline $1.19 \mathrm{~J} / \mathrm{cm}^{2}$ & & $\begin{array}{l}\text { Backscatter, white spot } \\
\text { visible by eye and SPM. } \\
\text { Magnification I0x. }\end{array}$ \\
\hline $1.19 \mathrm{~J} / \mathrm{cm}^{2}$ & & $\begin{array}{c}\text { Backscatter, white spot } \\
\text { visible by eye. } \\
\text { Magnification I0x. }\end{array}$ \\
\hline $1.14 \mathrm{~J} / \mathrm{cm}^{2}$ & $\stackrel{\bullet}{4 \mu \mathrm{m}}$ & $\begin{array}{l}\text { No spectral broadening. } \\
\text { Magnification 40x. }\end{array}$ \\
\hline $1.11 \mathrm{~J} / \mathrm{cm}^{2}$ & $\frac{115 \mu \mathrm{m}}{115}$ & $\begin{array}{l}\text { Backscatter, white spot } \\
\text { visible by eye and SPM. } \\
\text { Magnification 40x. }\end{array}$ \\
\hline $1.04 \mathrm{~J} / \mathrm{cm}^{2}$ & $4 \stackrel{2}{4 m}$ & $\begin{array}{l}\text { No spectral broadening. } \\
\text { Magnification 40x. }\end{array}$ \\
\hline
\end{tabular}

Figure A.3: The table shows different kinds of damages for different fluences. The pictures with a magnification of 40 were captured with a phase contrast microscope. 


\section{Appendix B}

\section{Matlab code}

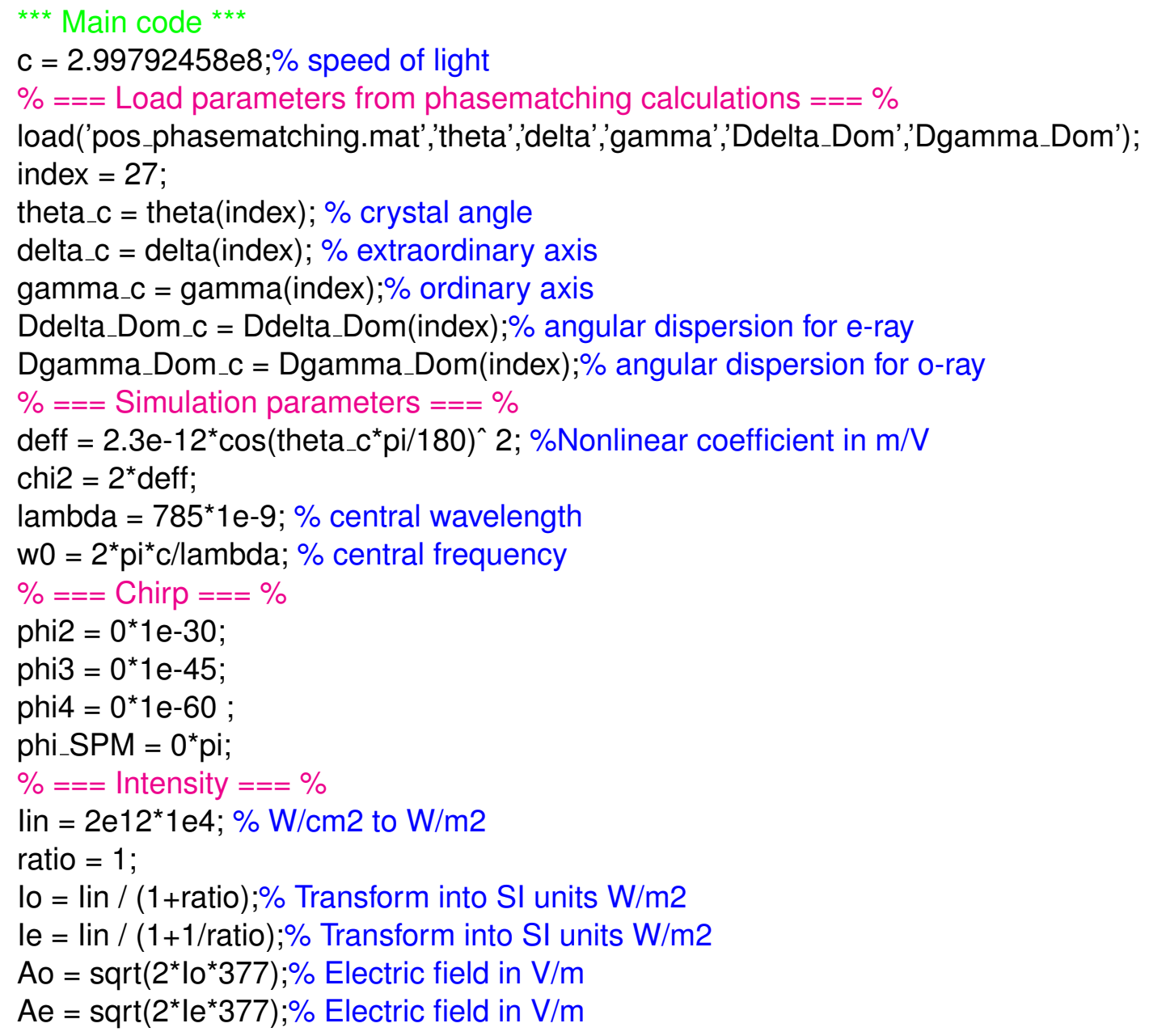




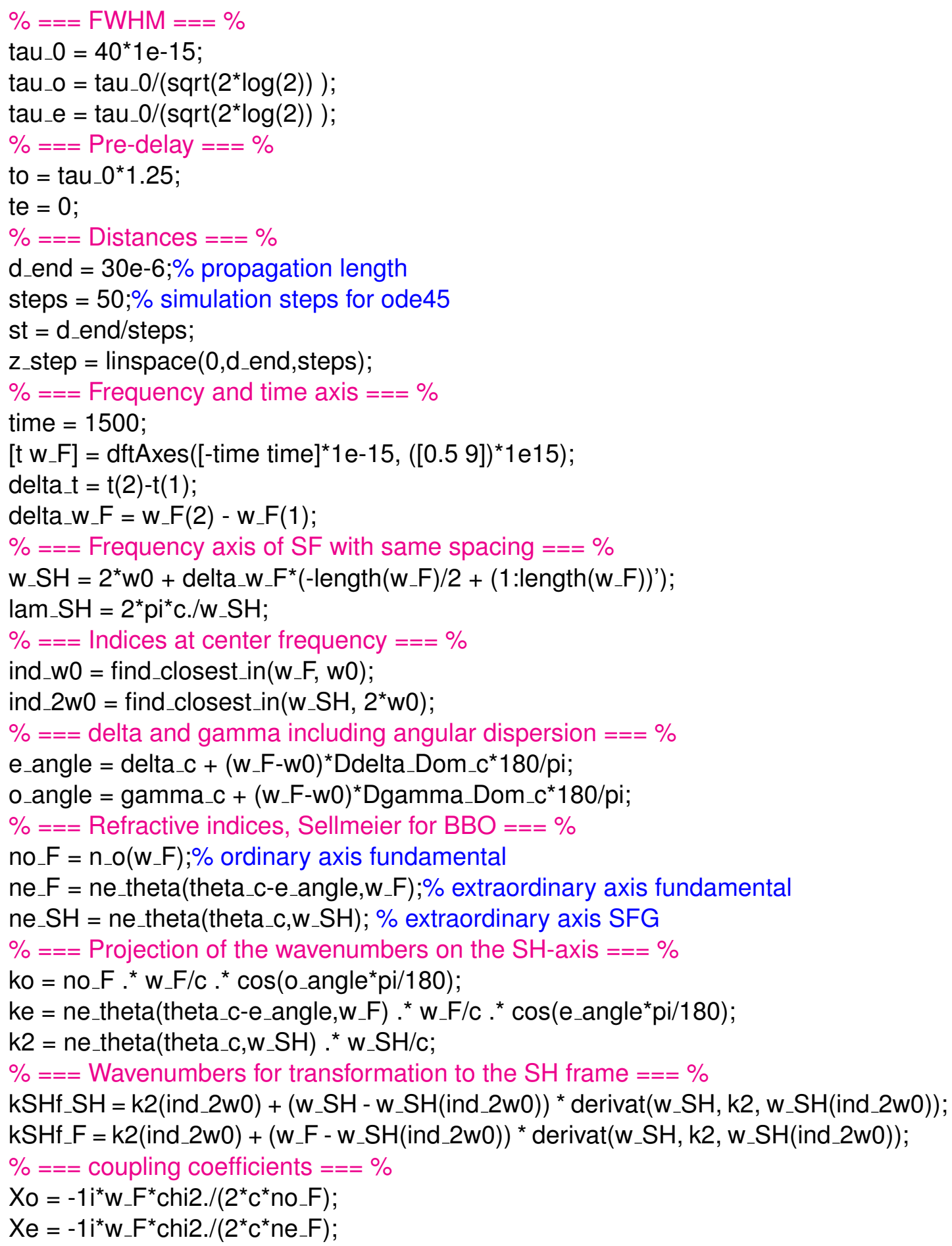


$\mathrm{X} 2=-1 \mathrm{i}^{*} \mathrm{w}_{-} \mathrm{SH}^{*} \mathrm{chi} 2 . /\left(2^{*} \mathrm{C}^{*}\right.$ ne_SH);

$\%===$ Defining the initial pulse envelopes in the frequecy domain $===\%$

Eo_f $=$ Ao*tau_o/sqrt $(2){ }^{*} \exp \left(1 i^{*}\left(\left(w_{-} \text {F-w } 0\right)^{*}\right.\right.$ to $+0.5^{*}\left(w_{-}\right.$F-w 0$) .{ }^{\star} 2^{*}$ phi $2+1 / 6^{*}\left(w_{-} F-\right.$ w0). $3^{*}$ phi3 + 1/24* (w_F-w0). $4^{*}$ phi4)) . ${ }^{*} \exp \left(-\left(\left(w_{-} F-w 0\right)^{*}\right.\right.$ tau_o/2). $\left.{ }^{\wedge} 2\right)$;

Ee_f $=A e^{*}$ tau_e $/$ sqrt $(2){ }^{*} \exp \left(1 i^{*}\left(\left(w_{-} F-w 0\right)^{*} t e+0.5^{*}\left(w_{-} F-w 0\right) .{ }^{\wedge} 2^{*}\right.\right.$ phi $2+1 / 6^{*}\left(w_{-} F-\right.$ w0). ${ }^{\wedge} 3^{*}$ phi3 $+1 / 24^{*}\left(w_{-} F-w 0\right) .{ }^{\wedge}{ }^{*}$ phi4 $\left.)\right) .{ }^{*} \exp \left(-\left(\left(w_{-} F-w 0\right)^{*}\right.\right.$ tau_e/2). $\left.{ }^{\wedge} 2\right)$;

E2_f $=$ zeros $\left(\right.$ length $\left.\left(w_{-} S \mathrm{SH}\right), 1\right)$;

$\%===\mathrm{FT}$ to temporal domain $===\%$

Eo_t0 $=\operatorname{dftv}\left(w_{-} F, 1\right.$, Eo_f $\left.t\right)$;

$E_{-} \_t 0=\operatorname{dftv}\left(w_{-} F, 1, E_{-} f, t\right) ;$

$\%==$ Nonlinear phase $==\%$

Eo_t0 $=$ Eo_t0. ${ }^{*} \exp \left(1 i^{*}\right.$ normMax $(\text { abs_sqd }(\text { Eo_t0 }))^{*}$ phi_SPM $)$;

Eo_f $=\operatorname{dftv}\left(t,-1\right.$, Eo_t0, $\left.w_{-} F\right)$;

Ee_t0 $=$ Ee_t0. ${ }^{*} \exp \left(1 i^{*}\right.$ normMax $\left(\text { abs_sqd }\left(E E_{\_} \_0\right)\right)^{*}$ phi_SPM $)$;

Ee_f $=\operatorname{dftv}\left(t,-1, E_{-} t 0, w_{-} F\right)$

$\%===$ Starting conditions $===\%$

Eoe2=[Eo_f.' Ee_f.' E2_f.'].';

$\%===$ ODE45 === \%

opts = odeset('RelTol',1e-7,'AbsTol',1e-9);

[z_ode,E_ode] = ode45(@coupled,z_step,Eoe2, opts, t,w_F,w_SH,ko,ke,k2,Xo,Xe,X2);

$\%===$ Reshape into 3 Matrices of $t$ elements $===\%$

Eo_ode_f = E_ode(:, 1 :length $(\mathrm{t}))$.';

Ee_ode_f = E_ode $(:$, length $(\mathrm{t})+(1$ :length $(\mathrm{t})))$;;

E2_ode_f $=$ E_ode $\left(:\right.$, length $(\mathrm{t})^{\star} 2+(1$ :length $\left.(\mathrm{t}))\right)$.;

$\%===\mathrm{FT}$ to temporal domain $===\%$

Eo_ode_t $=\operatorname{dftv}\left(w_{-}\right.$F, 1,Eo_ode_f,t $)$;

Ee_ode_t $=\operatorname{dftv}\left(w_{-}+F, 1, E e_{-}\right.$ode_f $\left.t\right)$;

E2_ode_t $=\operatorname{dftv}\left(w_{-} \_\mathrm{SH}, 1, \mathrm{E} 2 \_\right.$ode_f,t $)$;

$\%===$ Convert to $\mathrm{SH}$ frame $===\%$

Eo_SHf_f = Eo_ode_f .* $\exp \left(-1 i^{*} b s x f u n(@\right.$ times, ko - kSHf_F, z_ode'));

Ee_SHf_f = Ee_ode_f .* $\exp \left(-1 i^{*}\right.$ bsxfun(@times, ke - kSHf_F, z_ode'));

E2_SHf_f = E2_ode_f .* exp(-1ibsxfun(@times, k2 - kSHf_SH, z_ode'));

$\%===\mathrm{FT}$ to temporal domain $===\%$

Eo_SHf_t $=\operatorname{dftv}\left(w_{-} F, 1\right.$, Eo_SHf_f, $\left.t\right)$;

Ee_SHf_t $=\operatorname{dftv}\left(w_{-}+F, 1, E_{-}\right.$SHf $\left._{-}, t\right)$;

$E 2 \_S H f \_t=\operatorname{dftv}\left(w_{-} S H, 1, E 2 \_S H f \_f, t\right)$;

*** Function used for ode45 ***

function [dE]=coupled(z,E,t,w_F,w_SH,ko,ke,k2,Xo,Xe,X2)

$\%$ Convert single row vector to 3 separate vector fields 
size $\_$E $=$size $(E)$;

$E=\operatorname{reshape}\left(E_{\text {, size }} E(1) / 3,3\right)$;

Eo_f $=E(:, 1)$;

$E_{-} f=E(:, 2)$;

$E 2 \_f=E(:, 3)$;

$\%$ Fourier from freq to time $+z$ dependence

$E 2 \_t=\operatorname{dftv}\left(w_{-}-S H, 1, E 2 f_{-}{ }^{*} \exp \left(-1 i^{*} k 2^{*} z\right), t\right)$;

Eo_t $=\operatorname{dftv}\left(w_{-} F, 1\right.$, Eo_f. $\left.{ }^{*} \exp \left(-1 i^{*} k o^{*} z\right), t\right)$;

Ee_t $=\operatorname{dftv}\left(w_{-} F, 1, E_{-} f^{*}{ }^{*} \exp \left(-1 i^{*} k e^{*} z\right), t\right)$;

$\%$ Perform ode 45 on coupled equations

$\mathrm{dE2dz}=\mathrm{X} 2 .{ }^{*} \mathrm{dftv}\left(\mathrm{t},-1\right.$, Eo_t. ${ }^{*}$ Ee_t, w_SH $) .{ }^{*} \exp \left(1 \mathrm{i}^{*} \mathrm{k} 2^{*} \mathrm{z}\right)$;

$\mathrm{dEodz}=$ Xo. ${ }^{*} \operatorname{dftv}\left(\mathrm{t},-1, \mathrm{E}_{-} \mathrm{tt}^{*}{ }^{*} \operatorname{conj}\left(\mathrm{Ee}_{\mathrm{t}} \mathrm{t}\right), \mathrm{w}_{-} \mathrm{F}\right) .{ }^{*} \exp \left(1 \mathrm{i}^{*} \mathrm{k} \mathrm{o}^{*} \mathrm{z}\right)$;

$\mathrm{dEedz}=$ Xe. ${ }^{*} \operatorname{dftv}\left(\mathrm{t},-1, \mathrm{E} 2 \_\mathrm{t} .{ }^{*} \operatorname{conj}\left(E_{0} \_\mathrm{t}\right), \mathrm{w}_{-} \mathrm{F}\right) .{ }^{*} \exp \left(1 \mathrm{i}^{*} \mathrm{ke}{ }^{*} \mathrm{z}\right)$;

$\mathrm{dE}=[\mathrm{dEodz} ; \mathrm{dEedz} ; \mathrm{dE2} \mathrm{dz}]$ 


\section{Acronyms}

ASE amplified spontaneous emission.

BBO $\beta$-Barium Borate.

BFD broadband frequency doubling.

CE carrier-envelope.

CEP carrier-envelope phase.

CPA chirped pulse amplification.

DFG difference-frequency generation.

DUV deep ultraviolet.

FFT fast Fourier transform.

FROG frequency-resolved optical gating.

FWHM full width half maximum.

GDD group-delay dispersion.

GVD group-velocity dispersion.

GVM group-velocity mismatch.

HCF hollow core fibre.

HHG high-order harmonic generation.

LIDT laser induced damage threshold. 
MIR mid-infrared.

NIR near-infrared.

NOPA noncollinear optical parametric amplifier.

NPC nonlinear pulse compression.

OPA optical parametric amplification.

OPCPA optical parametric chirped pulse amplification.

PFT pulse-front tilt.

PWG planar waveguide.

SD self-diffraction.

SD-FROG self-diffraction FROG.

SF sum-frequency.

SFG sum-frequency generation.

SH second harmonic.

SHG second-harmonic generation.

SHG-FROG second-harmonic generation FROG.

SPIDER spectral phase interferometry for direct electric-field reconstruction.

SPM self-phase modulation.

TOD third order dispersion.

UV ultraviolet.

XPM cross-phase modulation.

XUV extreme ultraviolet. 


\section{References}

[1] E. Goulielmakis, M. Schultze, M. Hofstetter, V. S. Yakovlev, J. Gagnon, M. Uiberacker, A. L. Aquila, E. M. Gullikson, D. T. Attwood, R. Kienberger, F. Krausz, and U. Kleineberg, "Single-Cycle Nonlinear Optics," Science 320(5883), 1614-1617 (2008).

[2] D. H. Ko, K. T. Kim, J. Park, J. hwan Lee, and C. H. Nam, "Attosecond chirp compensation over broadband high-order harmonics to generate near transform-limited 63as pulses," New J. Phys. 12(6), 063,008 (2010).

[3] Z. Chang, "Generation of Single Isolated 67-Attosecond Pulses," in Laser Science, p. LW1H.1 (Optical Society of America, 2012).

[4] X. Zhou, T. Kanai, D. Yoshitomi, T. Sekikawa, and S. Watanabe, "Generation of high average power, 7.5 -fs blue pulses at $5 \mathrm{kHz}$ by adaptive phase control," Appl. Phys. B-Lasers O. 81, 13-17 (2005).

[5] M. Nisoli, S. D. Silvestri, O. Svelto, R. Szipöcs, K. Ferencz, C. Spielmann, S. Sartania, and F. Krausz, "Compression of high-energy laser pulses below 5 fs," Opt. Lett. 22(8), 522-524 (1997).

[6] C. Hauri, W. Kornelis, F. Helbing, A. Heinrich, A. Couairon, A. Mysyrowicz, J. Biegert, and U. Keller, "Generation of intense, carrier-envelope phaselocked few-cycle laser pulses through filamentation," Appl. Phys. B-Lasers O. 79(6), 673-677 (2004).

[7] S. Bohman, A. Suda, T. Kanai, S. Yamaguchi, and K. Midorikawa, "Generation of $5.0 \mathrm{fs}, 5.0 \mathrm{~mJ}$ pulses at $1 \mathrm{kHz}$ using hollow-fiber pulse compression," Opt. Lett. 35(11), 1887-1889 (2010).

[8] S. Akturk, C. L. Arnold, B. Zhou, and A. Mysyrowicz, "High-energy ultrashort laser pulse compression in hollow planar waveguides," Opt. Lett. 34(9), 1462-1464 (2009).

[9] J. Chen, A. Suda, E. J. Takahashi, M. Nurhuda, and K. Midorikawa, "Compression of intense ultrashort laser pulses in a gas-filled planar waveguide," Opt. Lett. 33(24), 2992-2994 (2008).

[10] S. Chen, A. Jarnac, A. Houard, Y. Liu, C. L. Arnold, B. Zhou, B. Forestier, B. Prade, and A. Mysyrowicz, "Compression of high-energy ultrashort laser pulses through an argon-filled tapered planar waveguide," J. Opt. Soc. Am. B 28(5), 1009-1012 (2011). 
[11] Y. Wang and R. Dragila, "Efficient conversion of picosecond laser pulses into second-harmonic frequency using group-velocity dispersion," Phys. Rev. A 41(10), 5645-5649 (1990).

[12] A. Stabinis, G. Valiulis, and E. Ibragimov, "Effective sum frequency pulse compression in nonlinear crystals," Opt. Commun. 86(3-4), $301-306$ (1991).

[13] E. Treacy, "Optical Pulse Compression With Diffraction Gratings," IEEE J. Quantum Elect. QE 5(9), 454 (1969).

[14] O. E. Martinez, J. P. Gordon, and R. L. Fork, "Negative group-velocity dispersion using refraction," J. Opt. Soc. Am. A 1(10), 1003-1006 (1984).

[15] R. L. Fork, O. E. Martinez, and J. P. Gordon, "Negative dispersion using pairs of prisms," Opt. Lett. 9(5), 150-152 (1984).

[16] F. Gires and P. Tournois, "Interferometre utilisable pour la compression d'impulsions lumineuses modulées en frequence," C. R. Acad. Sci. 258(25), 6112 (1964).

[17] J. Giordmaine, M. Duguay, and J. Hansen, "Compression of optical pulses," IEEE J. Quantum Elect. 4(5), 252 - 255 (1968).

[18] R. Boyd, Nonlinear optics, Electronics \& Electrical (Academic Press, 2003).

[19] H. Nakatsuka and D. Grischkowsky, "Recompression of optical pulses broadened by passage through optical fibers," Opt. Lett. 6(1), 13-15 (1981).

[20] H. Nakatsuka, D. Grischkowsky, and A. C. Balant, "Nonlinear PicosecondPulse Propagation through Optical Fibers with Positive Group Velocity Dispersion," Phys. Rev. Lett. 47, 910-913 (1981).

[21] C. V. Shank, R. L. Fork, R. Yen, R. H. Stolen, and W. J. Tomlinson, "Compression of femtosecond optical pulses," Appl. Phys. Lett. 40(9), 761-763 (1982).

[22] R. L. Fork, C. H. B. Cruz, P. C. Becker, and C. V. Shank, "Compression of optical pulses to six femtoseconds by using cubic phase compensation," Opt. Lett. 12(7), 483-485 (1987).

[23] A. Baltuška, Z. Wei, M. S. Pshenichnikov, and D. A. Wiersma, "Optical pulse compression to 5 fs at a $1-\mathrm{MHz}$ repetition rate," Opt. Lett. 22(2), 102-104 (1997).

[24] M. Nisoli, S. D. Silvestri, and O. Svelto, "Generation of high energy 10 fs pulses by a new pulse compression technique," Appl. Phys. Lett. 68(20), 2793-2795 (1996).

[25] F. X. Kärtner, Few-cycle laser pulse generation and its applications, Topics in applied physics (Springer, 2004.).

[26] G. Cerullo, S. De Silvestri, M. Nisoli, S. Sartania, S. Stagira, and O. Svelto, "Few-optical-cycle laser pulses: From high peak power to frequency tunability," IEEE J. Sel. Top. Quantum Electron. 6(6), 948-958 (2000). 
[27] B. Schenkel, J. Biegert, U. Keller, C. Vozzi, M. Nisoli, G. Sansone, S. Stagira, S. D. Silvestri, and O. Svelto, "Generation of 3.8-fs pulses from adaptive compression of a cascaded hollow fiber supercontinuum," Opt. Lett. 28(20), 1987-1989 (2003).

[28] E. Matsubara, K. Yamane, T. Sekikawa, and M. Yamashita, "Generation of 2.6 fs optical pulses using induced-phase modulation in a gas-filled hollow fiber," J. Opt. Soc. Am. B 24(4), 985-989 (2007).

[29] A. Suda, M. Hatayama, K. Nagasaka, and K. Midorikawa, "Generation of sub-10-fs, 5-mJ-optical pulses using a hollow fiber with a pressure gradient," Appl. Phys. Lett. 86(11), 111116 (2005).

[30] Y. Oishi, A. Suda, K. Midorikawa, and F. Kannari, "Sub-10 fs, multimillijoule laser system," Rev. Sci. Instrum. 76(9), 093114 (2005).

[31] B. Shan, S. Ghimire, and Z. Chang, "Generation of the attosecond extreme ultraviolet supercontinuum by a polarization gating," J. Mod. Optic. 52(2-3), 277-283 (2005).

[32] J. Sung, J. Park, T. Imran, Y. Lee, and C. Nam, "Generation of 0.2-TW 5.5-fs optical pulses at $1 \mathrm{kHz}$ using a differentially pumped hollow-fiber chirpedmirror compressor," Appl. Phys. B-Lasers 0. 82, 5-8 (2006).

[33] J. Park, J. hwan Lee, and C. H. Nam, "Generation of 1.5 cycle 0.3 TW laser pulses using a hollow-fiber pulse compressor," Opt. Lett. 34(15), 2342-2344 (2009).

[34] S. Bohman, A. Suda, M. Kaku, M. Nurhuda, T. Kanai, S. Yamaguchi, and K. Midorikawa, "Generation of 5 fs, 0.5 TW pulses focusable to relativistic intensities at $1 \mathrm{kHz}$," Opt. Express 16(14), 10,684-10,689 (2008).

[35] A. S. Wyatt, A. Grün, P. K. Bates, O. Chalus, J. Biegert, and I. A. Walmsley, "Accuracy measurements and improvement for complete characterization of optical pulses from nonlinear processes via multiple spectral-shearing interferometry," Opt. Express 19(25), 25,355-25,366 (2011).

[36] A. Braun, G. Korn, X. Liu, D. Du, J. Squier, and G. Mourou, "Self-channeling of high-peak-power femtosecond laser pulses in air," Opt. Lett. 20(1), 73-75 (1995).

[37] P. Sprangle, E. Esarey, and J. Krall, "Self-guiding and stability of intense optical beams in gases undergoing ionization," Phys. Rev. E 54(4), 42114232 (1996).

[38] A. Couairon, M. Franco, A. Mysyrowicz, J. Biegert, and U. Keller, "Pulse self-compression to the single-cycle limit by filamentation in a gas with a pressure gradient," Opt. Lett. 30(19), 2657-2659 (2005).

[39] A. Couairon, J. Biegert, C. P. Hauri, W. Kornelis, F. W. Helbing, U. Keller, and A. Mysyrowicz, "Self-compression of ultra-short laser pulses down to one optical cycle by filamentation," J. Mod. Optic. 53(1-2), 75-85 (2006). 
[40] G. Stibenz, N. Zhavoronkov, and G. Steinmeyer, "Self-compression of millijoule pulses to 7.8 fs duration in a white-light filament," Opt. Lett. 31(2), 274-276 (2006).

[41] X. Chen, X. Li, J. Liu, P. Wei, X. Ge, R. Li, and Z. Xu, "Generation of 5 fs, $0.7 \mathrm{~mJ}$ pulses at $1 \mathrm{kHz}$ through cascade filamentation," Opt. Lett. 32(16), 2402-2404 (2007).

[42] M. Hemmer, M. Baudisch, A. Thai, A. Couairon, and J. Biegert, "Selfcompression to sub-3-cycle duration of mid-infrared optical pulses in dielectrics," Opt. Express 21(23), 28,095-28,102 (2013).

[43] P. B. Corkum and F. Krausz, "Attosecond science," Nat. Phys. 3(6), 381-387 (2007).

[44] T. Brabec and F. Krausz, "Intense few-cycle laser fields: Frontiers of nonlinear optics," Rev. Mod. Phys. 72(2), 545-591 (2000).

[45] M. Schultze, M. Fieß, N. Karpowicz, J. Gagnon, M. Korbman, M. Hofstetter, S. Neppl, A. L. Cavalieri, Y. Komninos, T. Mercouris, C. A. Nicolaides, R. Pazourek, S. Nagele, J. Feist, J. Burgdörfer, A. M. Azzeer, R. Ernstorfer, R. Kienberger, U. Kleineberg, E. Goulielmakis, F. Krausz, and V. S. Yakovlev, "Delay in Photoemission," Science 328(5986), 1658-1662 (2010).

[46] U. Keller, "Recent developments in compact ultrafast lasers," Nature 424(6950), 831-838 (2003).

[47] M. Nurhuda, A. Suda, K. Midorikawa, and H. Budiono, "Control of selfphase modulation and plasma-induced blueshifting of high-energy, ultrashort laser pulses in an argon-filled hollow fiber using conjugate pressuregradient method," J. Opt. Soc. Am. B 22(8), 1757-1762 (2005).

[48] S. Ghimire, B. Shan, C. Wang, and Z. Chang, "High-energy 6.2-fs pulses for attosecond pulse generation," Laser Phys. 15(6), 838-842 (2005).

[49] X. Chen, A. Malvache, A. Ricci, A. Jullien, and R. Lopez-Martens, "Efficient hollow fiber compression scheme for generating multi-mJ, carrier-envelope phase stable, sub-5 fs pulses," Laser Phys. 21(1), 198-201 (2011).

[50] E. Schulz, T. Binhammer, D. Steingrube, S. Rausch, M. Kovacev, and U. Morgner, "Intense few-cycle laser pulses from self-compression in a selfguiding filament," Appl. Phys. B-Lasers O. 95, 269-272 (2009).

[51] D. Wang, X. W. Chen, X. F. Li, Y. X. Leng, R. X. Li, and Z. Z. Xu, "Generation of sub- 5 fs pulses through cascade of filamentation and hollow fiber compression," Laser Phys. Lett. 5(11), 830-833 (2008).

[52] M. Nurhuda, A. Suda, S. Bohman, S. Yamaguchi, and K. Midorikawa, "Optical Pulse Compression of Ultrashort Laser Pulses in an Argon-Filled Planar Waveguide," Phys. Rev. Lett. 97, 153,902 (2006).

[53] A. Guandalini, P. Eckle, M. Anscombe, P. Schlup, J. Biegert, and U. Keller, " 5.1 fs pulses generated by filamentation and carrier envelope phase stability analysis," J. Phys. B: At. Mol. Opt. Phys. 39(13), S257-S264 (2006). 
[54] J. Park, J. hwan Lee, and C. H. Nam, "Laser chirp effect on femtosecond laser filamentation generated for pulse compression," Opt. Express 16(7), 4465-4470 (2008).

[55] G. Sansone, G. Steinmeyer, C. Vozzi, S. Stagira, M. Nisoli, S. De Silvestri, K. Starke, D. Ristau, B. Schenkel, J. Biegert, A. Gosteva, and U. Keller, "Mirror dispersion control of a hollow fiber supercontinuum," Appl. Phys. BLasers O. 78, 551-555 (2004).

[56] A. Anderson, F. Lücking, T. Prikoszovits, M. Hofer, Z. Cheng, C. Neacsu, M. Scharrer, S. Rammler, P. Russell, G. Tempea, and A. Assion, "Multi-mJ carrier envelope phase stabilized few-cycle pulses generated by a tabletop laser system," Appl. Phys. B-Lasers O. 103, 531-536 (2011).

[57] D. A. Guk and V. G. Dmitriev, "Some characteristics of second harmonic generation under conditions of strong energy exchange between interacting waves," Sov. J. Quant. Electron. 21(1), 96 (1991).

[58] Y. Wang and B. Luther-Davies, "Frequency-doubling pulse compressor for picosecond high-power neodymium laser pulses," Opt. Lett. 17(20), 14591461 (1992).

[59] P. Heinz, A. Laubereau, A. Dubietis, and A. Piskarskas., "Fiberless twostep parametric compression of sub-picosecond laser pulses," Lith. J. Phys. 33(5-6), 314 (1993).

[60] C. Y. Chien, G. Korn, J. S. Coe, J. Squier, G. Mourou, and R. S. Craxton, "Highly efficient second-harmonic generation of ultraintense Nd:glass laser pulses," Opt. Lett. 20(4), 353-355 (1995).

[61] A. Umbrasas, J.-C. Diels, J. Jacob, G. Valiulis, and A. Piskarskas, "Generation of femtosecond pulses through second-harmonic compression of the output of a Nd:YAG laser," Opt. Lett. 20(21), 2228-2230 (1995).

[62] J. Biegert, V. Kubecek, and J. Diels, "Second harmonic pulse compression," Springer Series in Chemical Physics 63, 84-86 (1998).

[63] M. Nisoli, S. D. Silvestri, G. Valiulis, and A. Varanavicius, "Fivefold femtosecond pulse compression by sum frequency generation," Appl. Phys. Lett. 68(25), 3540-3542 (1996).

[64] A. Dubietis, G. Valiulis, G. Tamošauskas, R. Danielius, and A. Piskarskas, "Nonlinear second-harmonic pulse compression with tilted pulses," Opt. Lett. 22(14), 1071-1073 (1997).

[65] O. Martinez, "Achromatic phase matching for second harmonic generation of femtosecond pulses," IEEE J. Quantum Elect. 25(12), 2464 -2468 (1989).

[66] T. R. Zhang, H. R. Choo, and M. C. Downer, "Phase and group velocity matching for second harmonic generation of femtosecond pulses," Appl. Opt. 29(27), 3927-3933 (1990). 
[67] P. D. Trapani, A. Andreoni, C. Solcia, P. Foggi, R. Danielius, A. Dubietis, and A. Piskarskas, "Matching of group velocities in three-wave parametric interaction with femtosecond pulses and application to traveling-wave generators," J. Opt. Soc. Am. B 12(11), 2237-2244 (1993).

[68] G. M. Gale, M. Cavallari, T. J. Driscoll, and F. Hache, "Sub-20-fs tunable pulses in the visible from an 82-MHz optical parametric oscillator," Opt. Lett. 20(14), 1562-1564 (1995).

[69] A. Shirakawa, I. Sakane, and T. Kobayashi, "Pulse-front-matched optical parametric amplification for sub-10-fs pulse generation tunable in the visible and near infrared," Opt. Lett. 23(16), 1292-1294 (1998).

[70] T. Wilhelm, J. Piel, and E. Riedle, "Sub-20-fs pulses tunable across the visible from a blue-pumped single-pass noncollinear parametric converter," Opt. Lett. 22(19), 1494-1496 (1997).

[71] G. Cerullo, M. Nisoli, S. Stagira, and S. D. Silvestri, "Sub-8-fs pulses from an ultrabroadband optical parametric amplifier in the visible," Opt. Lett. 23(16), 1283-1285 (1998).

[72] J. Biegert and J. Diels, "Compression of pulses of a few optical cycles through harmonic generation," J. Opt. Soc. Am. B 18(8), 1218-1226 (2001).

[73] A. Dubietis, G. Valiulis, R. Danielius, and A. Piskarskas, "Fundamentalfrequency pulse compression through cascaded second-order processes in a type II phase-matched second-harmonic generator," Opt. Lett. 21(16), 1262-1264 (1996).

[74] L. Lefort and A. Barthelemy, "Intensity-dependent polarization rotation associated with type II phase-matched second-harmonic generation: application to self-induced transparency," Opt. Lett. 20(17), 1749-1751 (1995).

[75] A. Baltuška and T. Kobayashi, "Adaptive shaping of two-cycle visible pulses using a flexible mirror," Appl. Phys. B-Lasers O. 75, 427-443 (2002).

[76] G. Cerullo and S. D. Silvestri, "Ultrafast optical parametric amplifiers," Rev. Sci. Instrum. 74(1), 1-18 (2003).

[77] P. Baum, S. Lochbrunner, and E. Riedle, "Generation of tunable 7-fs ultraviolet pulses: achromatic phase matching and chirp management," Appl. Phys. B-Lasers O. 79, 1027-1032 (2004).

[78] C. G. Durfee III, S. Backus, H. C. Kapteyn, and M. M. Murnane, "Intense 8-fs pulse generation in the deep ultraviolet," Opt. Lett. 24(10), 697-699 (1999).

[79] Y. Kida, J. Liu, T. Teramoto, and T. Kobayashi, "Sub-10 fs deep-ultraviolet pulses generated by chirped-pulse four-wave mixing," Opt. Lett. 35(11), 1807-1809 (2010).

[80] Y. Kida and T. Kobayashi, "Generation of sub-10 fs ultraviolet Gaussian pulses," J. Opt. Soc. Am. B 28(1), 139-148 (2011). 
[81] Y. Kida, K. Okamura, J. Liu, and T. Kobayashi, "Sub-10-fs deep-ultraviolet light source with stable power and spectrum," Appl. Opt. 51(26), 6403-6410 (2012).

[82] T. Fuji and T. Suzuki, "Generation of sub-two-cycle mid-infrared pulses by four-wave mixing through filamentation in air," Opt. Lett. 32(22), 3330-3332 (2007).

[83] U. Graf, M. Fieß, M. Schultze, R. Kienberger, F. Krausz, and E. Goulielmakis, "Intense few-cycle light pulses in the deep ultraviolet," Opt. Express 16(23), 18,956-18,963 (2008).

[84] K. Kosma, S. A. Trushin, W. E. Schmid, and W. Fuß, "Vacuum ultraviolet pulses of 11 fs from fifth-harmonic generation of a Ti:sapphire laser," Opt. Lett. 33(7), 723-725 (2008).

[85] F. Reiter, U. Graf, M. Schultze, W. Schweinberger, H. Schröder, N. Karpowicz, A. M. Azzeer, R. Kienberger, F. Krausz, and E. Goulielmakis, "Generation of sub-3 fs pulses in the deep ultraviolet," Opt. Lett. 35(13), 2248-2250 (2010).

[86] S. A. Trushin, K. Kosma, W. Fuß, and W. E. Schmid, "Sub-10-fs supercontinuum radiation generated by filamentation of few-cycle $800 \mathrm{~nm}$ pulses in argon," Opt. Lett. 32(16), 2432-2434 (2007).

[87] O. Dühr, E. T. J. Nibbering, G. Korn, G. Tempea, and F. Krausz, "Generation of intense 8-fs pulses at $400 \mathrm{~nm}$," Opt. Lett. 24(1), 34-36 (1999).

[88] J. Liu, K. Okamura, Y. Kida, T. Teramoto, and T. Kobayashi, "Clean sub-8fs pulses at $400 \mathrm{~nm}$ generated by a hollow fiber compressor for ultraviolet ultrafast pump-probe spectroscopy," Opt. Express 18(20), 20,645-20,650 (2010).

[89] N. Zhavoronkov and G. Korn, "Generation of Single Intense Short Optical Pulses by Ultrafast Molecular Phase Modulation," Phys. Rev. Lett. 88, 203,901 (2002).

[90] T. Kanai, X. Zhou, T. Liu, A. Kosuge, T. Sekikawa, and S. Watanabe, "Generation of terawatt 10 -fs blue pulses by compensation for pulse-frontdistortion in broadband frequency doubling," Opt. Lett. 29(24), 2929-2931 (2004).

[91] Y.-X. Yan, J. Edward B. Gamble, and K. A. Nelson, "Impulsive stimulated scattering: General importance in femtosecond laser pulse interactions with matter, and spectroscopic applications," J. Chem. Phys. 83(11), 5391-5399 (1985).

[92] C. D'Amico, A. Houard, M. Franco, B. Prade, A. Mysyrowicz, A. Couairon, and V. T. Tikhonchuk, "Conical Forward THz Emission from FemtosecondLaser-Beam Filamentation in Air," Phys. Rev. Lett. 98, 235,002 (2007).

[93] Q. Luo, H. Xu, S. Hosseini, J.-F. Daigle, F. Théberge, M. Sharifi, and S. Chin, "Remote sensing of pollutants using femtosecond laser pulse fluorescence spectroscopy," Appl. Phys. B-Lasers O. 82, 105-109 (2006). 
References

[94] X. M. Zhao, P. Rambo, and J.-C. Diels, "Filamentation of femtosecond UV pulses in air," in Quantum Electronics and Laser Science Conference, p. QThD2 (Optical Society of America, 1995).

[95] Z. Zhang, X. Lu, T.-T. Xi, W.-X. Liang, Z.-Q. Hao, Y. Zhang, M.-L. Zhou, Z.-H. Wang, and J. Zhang, "Long distance filamentation of $400 \mathrm{~nm}$ femtosecond laser pulses in air," Appl. Phys. B-Lasers O. 97, 207-213 (2009).

[96] T. Nelson, T. Luk, A. Bernstein, and S. Cameron, "Laser filamentation of a femtosecond pulse in air at $400 \mathrm{~nm}$," in Quantum Electronics and Laser Science Conference, 2001. QELS '01. Technical Digest. Summaries of Papers Presented at the, pp. $261-262$ (2001).

[97] S. Tzortzakis, B. Lamouroux, A. Chiron, M. Franco, B. Prade, A. Mysyrowicz, and S. D. Moustaizis, "Nonlinear propagation of subpicosecond ultraviolet laser pulses in air," Opt. Lett. 25(17), 1270-1272 (2000).

[98] S. Tzortzakis, B. Lamouroux, A. Chiron, S. Moustaizis, D. Anglos, M. Franco, B. Prade, and A. Mysyrowicz, "Femtosecond and picosecond ultraviolet laser filaments in air: experiments and simulations," Opt. Commun. 197(1-3), 131 - 143 (2001).

[99] J. Schwarz, P. Rambo, J.-C. Diels, M. Kolesik, E. M. Wright, and J. V. Moloney, "Ultraviolet filamentation in air," Opt. Commun. 180(4-6), 383 390 (2000).

[100] P. Béjot, J. Kasparian, and J.-P. Wolf, "Dual-color co-filamentation in Argon," Opt. Express 16(18), 14,115-14,127 (2008).

[101] T. Brixner, N. Damrauer, P. Niklaus, and G. Gerber, "Photoselective adaptive femtosecond quantum control in the liquid phase," Nature 414(6859), 57-60 (2001).

[102] T. Kobayashi, T. Saito, and H. Ohtani, "Real-time spectroscopy of transition states in bacteriorhodopsin during retinal isomerization," Nature 414(6863), 531-534 (2001).

[103] A. H. Zewail, "Femtochemistry: Atomic-scale dynamics of the chemical bond," J. Phys. Chem. A 104(24), 5660-5694 (2000).

[104] D. Polli, M. Antognazza, D. Brida, G. Lanzani, G. Cerullo, and S. D. Silvestri, "Broadband pump-probe spectroscopy with sub-10-fs resolution for probing ultrafast internal conversion and coherent phonons in carotenoids," Chem. Phys. 350(1-3), $45-55$ (2008).

[105] T. Suzuki, "Femtosecond Time-Resolved Photoelectron Imaging," Annu. Rev. Phys. Chem. 57(1), 555-592 (2006).

[106] T. Kobayashi and Y. Kida, "Ultrafast spectroscopy with sub-10 fs deepultraviolet pulses," Phys. Chem. Chem. Phys. 14, 6200-6210 (2012).

[107] P. B. Corkum, "Plasma perspective on strong field multiphoton ionization," Phys. Rev. Lett. 71(13), 1994-1997 (1993). 
[108] M. Lewenstein, P. Balcou, M. Y. Ivanov, A. L'Huillier, and P. B. Corkum, "Theory of high-harmonic generation by low-frequency laser fields," Phys. Rev. A 49, 2117-2132 (1994).

[109] B. Shan and Z. Chang, "Dramatic extension of the high-order harmonic cutoff by using a long-wavelength driving field," Phys. Rev. A 65, 011,804 (2001).

[110] J. Tate, T. Auguste, H. G. Muller, P. Salieres, P. Agostini, and L. F. DiMauro, "Scaling of Wave-Packet Dynamics in an Intense Midinfrared Field," Phys. Rev. Lett. 98(1), 013901 (2007).

[111] E. J. Takahashi, T. Kanai, Y. Nabekawa, and K. Midorikawa, "10 mJ class femtosecond optical parametric amplifier for generating soft x-ray harmonics," Appl. Phys. Lett. 93(4), 041111 (2008).

[112] D. R. Austin and J. Biegert, "Strong-field approximation for the wavelength scaling of high-harmonic generation," Phys. Rev. A 86, 023,813 (2012).

[113] T. Popmintchev, M.-C. Chen, D. Popmintchev, P. Arpin, S. Brown, S. Ališauskas, G. Andriukaitis, T. Balčiunas, O. D. Mücke, A. Pugzlys, A. Baltuška, B. Shim, S. E. Schrauth, A. Gaeta, C. Hernández-García, L. Plaja, A. Becker, A. Jaron-Becker, M. M. Murnane, and H. C. Kapteyn, "Bright Coherent Ultrahigh Harmonics in the keV X-ray Regime from MidInfrared Femtosecond Lasers," Science 336(6086), 1287-1291 (2012).

[114] K. Schiessl, K. L. Ishikawa, E. Persson, and J. Burgdörfer, "Quantum Path Interference in the Wavelength Dependence of High-Harmonic Generation," Phys. Rev. Lett. 99, 253,903 (2007).

[115] P. Colosimo, G. Doumy, C. I. Blaga, J. Wheeler, C. Hauri, F. Catoire, J. Tate, R. Chirla, A. M. March, G. G. Paulus, H. G. Muller, P. Agostini, and L. F. DiMauro, "Scaling strong-field interactions towards the classical limit," Nat. Phys. 4(5), 386-389 (2008).

[116] E. L. Falcão-Filho, V. M. Gkortsas, A. Gordon, and F. X. Kärtner, "Analytic scaling analysis of high harmonic generation conversion efficiency," Opt. Express 17(13), 11,217-11,229 (2009).

[117] E. L. Falcão-Filho, C.-J. Lai, K.-H. Hong, V.-M. Gkortsas, S.-W. Huang, L.-J. Chen, and F. X. Kärtner, "Scaling of high-order harmonic efficiencies with visible wavelength drivers: A route to efficient extreme ultraviolet sources," Appl. Phys. Lett. 97(6), 061,107 (2010).

[118] D. Bratton, D. Yang, J. Dai, and C. K. Ober, "Recent progress in high resolution lithography," Polym. Adv. Technol. 17(2), 94-103 (2006).

[119] S. Wurm, "Lithography development and research challenges for the $\leq$ 22nm half-pitch," 25th European Mask and Lithography Conference 7470(1), 747002 (2009).

[120] C. Wagner and N. Harned, "EUV Lithography: Lithography gets extreme," Nat. Photonics 4(1), 24-26 (2010). 
[121] B. W. J. McNeil, J. A. Clarke, D. J. Dunning, G. J. Hirst, H. L. Owen, N. R. Thompson, B. Sheehy, and P. H. Williams, "An XUV-FEL amplifier seeded using high harmonic generation," New J. Phys. 9(4), 82 (2007).

[122] M. Mittenzwey, "High Harmonic Generation for the XUV seeding experiment at FLASH," Ph.D. thesis, Universität Hamburg (2011).

[123] A. Willner, M. Schulz, R. Riedel, M. Yeung, T. Dzelzainis, C. Kamperidis, M. Bakarezos, D. Adams, V. Yakovlev, J. Ronsch-Schulenburg, B. Dromey, N. Papadogiannis, M. Tatarakis, S. Dusterer, S. Schreiber, B. Faatz, M. Drescher, J. Rossbach, M. Zepf, and F. Tavella, "A new XUV-source for seeding a FEL at high repetition rates," SPIE Proceedings $8075(1), 80,750 \mathrm{~L}$ (2011).

[124] P. H. Bucksbaum, "The Future of Attosecond Spectroscopy," Science 317(5839), 766-769 (2007).

[125] A. L. Cavalieri, N. Muller, T. Uphues, V. S. Yakovlev, A. Baltuška, B. Horvath, B. Schmidt, L. Blumel, R. Holzwarth, S. Hendel, M. Drescher, U. Kleineberg, P. M. Echenique, R. Kienberger, F. Krausz, and U. Heinzmann, "Attosecond spectroscopy in condensed matter," Nature 449(7165), 1029-1032 (2007).

[126] F. Krausz and M. Ivanov, "Attosecond physics," Rev. Mod. Phys. 81(1), 163 (2009).

[127] Q. Peng, A. Juzeniene, J. Chen, L. O. Svaasand, T. Warloe, K.-E. Giercksky, and J. Moan, "Lasers in medicine," Rep. Prog. Phys. 71(5), 056,701 (2008).

[128] F. Rotermund and V. Petrov, "Femtosecond Noncollinear Optical Parametric Amplification in the Mid-Infrared Range with 1.25 um Pumping," Jpn. J. Appl. Phys. 40(Part 1, No. 5A), 3195-3200 (2001).

[129] C. Schriever, S. Lochbrunner, P. Krok, and E. Riedle, "Tunable pulses from below 300 to $970 \mathrm{~nm}$ with durations down to 14 fs based on a $2 \mathrm{MHz}$ ytterbium-doped fiber system," Opt. Lett. 33(2), 192-194 (2008).

[130] C. Vozzi, G. Cirmi, C. Manzoni, E. Benedetti, F. Calegari, G. Sansone, S. Stagira, O. Svelto, S. D. Silvestri, M. Nisoli, and G. Cerullo, "High-energy, few-optical-cycle pulses at $1.5 \mu \mathrm{m}$ with passive carrier-envelope phase stabilization," Opt. Express 14(21), 10,109-10,116 (2006).

[131] G. Cirmi, C. Manzoni, D. Brida, S. D. Silvestri, and G. Cerullo, "Carrierenvelope phase stable, few-optical-cycle pulses tunable from visible to near IR," J. Opt. Soc. Am. B 25(7), B62-B69 (2008).

[132] C. Zhang, P. Wei, Y. Huang, Y. Leng, Y. Zheng, Z. Zeng, R. Li, and Z. Xu, "Tunable phase-stabilized infrared optical parametric amplifier for high-order harmonic generation," Opt. Lett. 34(18), 2730-2732 (2009).

[133] D. Brida, G. Cirmi, C. Manzoni, S. Bonora, P. Villoresi, S. D. Silvestri, and G. Cerullo, "Sub-two-cycle light pulses at $1.6 \mu \mathrm{m}$ from an optical parametric amplifier," Opt. Lett. 33(7), 741-743 (2008). 
[134] C. Vozzi, F. Calegari, E. Benedetti, S. Gasilov, G. Sansone, G. Cerullo, M. Nisoli, S. D. Silvestri, and S. Stagira, "Millijoule-level phase-stabilized few-optical-cycle infrared parametric source," Opt. Lett. 32(20), 2957-2959 (2007).

[135] F. Rotermund, V. Petrov, F. Noack, M. Wittmann, and G. Korn, "Laserdiode-seeded operation of a femtosecond optical parametric amplifier with $\mathrm{MgO}: \mathrm{LiNbO} 3$ and generation of 5-cycle pulses near $3 \mu \mathrm{m}$," J. Opt. Soc. Am. B 16(9), 1539-1545 (1999).

[136] A. Dubietis, G. Jonušauskas, and A. Piskarskas, "Powerful femtosecond pulse generation by chirped and stretched pulse parametric amplification in BBO crystal," Opt. Commun. 88(4-6), 437 - 440 (1992).

[137] I. Ross, P. Matousek, M. Towrie, A. Langley, and J. Collier, "The prospects for ultrashort pulse duration and ultrahigh intensity using optical parametric chirped pulse amplifiers," Opt. Commun. 144(1-3), 125 - 133 (1997).

[138] O. V. Chekhlov, J. L. Collier, I. N. Ross, P. K. Bates, M. Notley, C. HernandezGomez, W. Shaikh, C. N. Danson, D. Neely, P. Matousek, S. Hancock, and L. Cardoso, "35 J broadband femtosecond optical parametric chirped pulse amplification system," Opt. Lett. 31(24), 3665-3667 (2006).

[139] V. V. Lozhkarev, G. I. Freidman, V. N. Ginzburg, E. V. Katin, E. A. Khazanov, A. V. Kirsanov, G. A. Luchinin, A. N. Mal'shakov, M. A. Martyanov, O. V. Palashov, A. K. Poteomkin, A. M. Sergeev, A. A. Shaykin, I. V. Yakovlev, S. G. Garanin, S. A. Sukharev, N. N. Rukavishnikov, A. V. Charukhchev, R. R. Gerke, and V. E. Yashin, "200 TW 45 fs laser based on optical parametric chirped pulse amplification," Opt. Express 14(1), 446-454 (2006).

[140] S. Adachi, N. Ishii, T. Kanai, A. Kosuge, J. Itatani, Y. Kobayashi, D. Yoshitomi, K. Torizuka, and S. Watanabe, " 5 -fs, multi-mJ, CEP-locked parametric chirped-pulse amplifier pumped by a $450-\mathrm{nm}$ source at $1 \mathrm{kHz}$," Opt. Express 16(19), 14,341-14,352 (2008).

[141] T. Fuji, N. Ishii, C. Y. Teisset, X. Gu, T. Metzger, A. Baltuška, N. Forget, D. Kaplan, A. Galvanauskas, and F. Krausz, "Parametric amplification of few-cycle carrier-envelope phase-stable pulses at $2.1 \mu \mathrm{m}$," Opt. Lett. 31(8), 1103-1105 (2006).

[142] O. Chalus, A. Thai, P. K. Bates, and J. Biegert, "Six-cycle mid-infrared source with $3.8 \mu \mathrm{J}$ at $100 \mathrm{kHz}$," Opt. Lett. 35(19), 3204-3206 (2010).

[143] D. Faccio, A. Grün, P. K. Bates, O. Chalus, and J. Biegert, "Optical amplification in the near-infrared in gas-filled hollow-core fibers," Opt. Lett. 34(19), 2918-2920 (2009).

[144] J. Colombelli, R. Pepperkok, E. H. K. Stelzer, and E. G. Reynaud, "Laser nanosurgery in cell biology," Med. Sci. 22(6-7), 651-658 (2006). 
[145] K. Namjou, C. Roller, T. Reich, J. Jeffers, G. McMillen, P. McCann, and M. Camp, "Determination of exhaled nitric oxide distributions in a diverse sample population using tunable diode laser absorption spectroscopy," Appl. Phys. B-Lasers O. 85(2-3), 427-435 (2006).

[146] P. J. Mazzone, "Analysis of volatile organic compounds in the exhaled breath for the diagnosis of lung cancer." J. Thorac. Oncol. 3(7), 774-780 (2008).

[147] F. K. Tittel, D. Richter, and A. Fried, "Mid-Infrared Laser Applications in Spectroscopy," (2003).

[148] B. Sheehy, J. D. D. Martin, L. F. DiMauro, P. Agostini, K. J. Schafer, M. B. Gaarde, and K. C. Kulander, "High Harmonic Generation at Long Wavelengths," Phys. Rev. Lett. 83(25), 5270-5273 (1999).

[149] A. Gordon and F. X. Kärtner, "Scaling of keV HHG photon yield with drive wavelength," Opt. Express 13(8), 2941-2947 (2005).

[150] M. J. Lighthill, “Group Velocity," IMA J. Appl. Math. 1(1), 1-28 (1965).

[151] S. Akturk, X. Gu, P. Gabolde, and R. Trebino, "The general theory of firstorder spatio-temporal distortions of Gaussian pulses and beams," Opt. Express 13(21), 8642-8661 (2005).

[152] S. Akturk, X. Gu, E. Zeek, and R. Trebino, "Pulse-front tilt caused by spatial and temporal chirp," Opt. Express 12(19), 4399-4410 (2004).

[153] J. Hebling, "Derivation of the pulse front tilt caused by angular dispersion," Opt. Quant. Electron. 28, 1759-1763 (1996).

[154] Z. Bor, B. Racz, G. Szabo, M. Hilbert, and H. A. Hazim, "Femtosecond pulse front tilt caused by angular dispersion," Optical Engineering 32(10), 2501-2504 (1993).

[155] K. Kato, "Second-harmonic generation to $2048 \AA$ in $\beta$-BaB2O4," IEEE J. Quantum Elect. 22(7), 1013 - 1014 (1986).

[156] F. Zernike and J. Midwinter, Applied nonlinear optics, Wiley series in pure and applied optics (Wiley, 1973).

[157] D. Eimerl, L. Davis, S. Velsko, E. K. Graham, and A. Zalkin, "Optical, mechanical, and thermal properties of barium borate," J. Appl. Phys. 62(5), 1968-1983 (1987).

[158] D. N. Nikogosyan, "Beta barium borate (BBO)," Appl. Phys. A-Mater. 52, 359-368 (1991).

[159] I. Shoji, H. Nakamura, K. Ohdaira, T. Kondo, R. Ito, T. Okamoto, K. Tatsuki, and S. Kubota, "Absolute measurement of second-order nonlinear-optical coefficients of $\beta$-BaB2O4 for visible to ultraviolet second-harmonic wavelengths," J. Opt. Soc. Am. B 16(4), 620-624 (1999).

[160] J. Diels and W. Rudolph, Ultrashort Laser Pulse Phenomena, Optics and photonics (Elsevier Science, 2006). 
[161] S. L. Cousin, N. Forget, A. Grün, P. K. Bates, D. R. Austin, and J. Biegert, "Few-cycle pulse characterization with an acousto-optic pulse shaper," Opt. Lett. 36(15), 2803-2805 (2011).

[162] F. Shimizu, "Frequency Broadening in Liquids by a Short Light Pulse," Phys. Rev. Lett. 19, 1097-1100 (1967).

[163] K. Wall and A. Sanchez, "Titanium Sapphire Lasers," Lincoln Lab. J. 3(3), 447-462 (1990).

[164] D. Strickland and G. Mourou, "Compression of amplified chirped optical pulses," Opt. Commun. 56(3), 219 - 221 (1985).

[165] Kapteyn-Murnane Laboratories Inc., Operator's Manual Red Dragon TM UItrafast Laser Amplifier System (2007).

[166] D. E. Spence, P. N. Kean, and W. Sibbett, "60-fsec pulse generation from a self-mode-locked Ti:sapphire laser," Opt. Lett. 16(1), 42-44 (1991).

[167] T. Brabec, C. Spielmann, P. F. Curley, and F. Krausz, "Kerr lens mode locking," Opt. Lett. 17(18), 1292-1294 (1992).

[168] J. Squier, F. Salin, G. Mourou, and D. Harter, "100-fs pulse generation and amplification in Ti:Al2O3," Opt. Lett. 16(5), 324-326 (1991).

[169] D. J. Kane and R. Trebino, "Single-shot measurement of the intensity and phase of an arbitrary ultrashort pulse by using frequency-resolved optical gating," Opt. Lett. 18(10), 823-825 (1993).

[170] R. Trebino, K. DeLong, D. Fittinghoff, J. Sweetser, M. Krumbugel, B. Richman, and D. Kane, "Measuring ultrashort laser pulses in the time-frequency domain using frequency-resolved optical gating," Rev. Sci. Instrum. 68(9), 3277-3295 (1997).

[171] R. Trebino, Frequency-Resolved Optical Gating: The Measurement of UItrashort Laser Pulses (Kluwer Academic, 2000).

[172] C. laconis and I. Walmsley, "Spectral phase interferometry for direct electricfield reconstruction of ultrashort optical pulses," Opt. Lett. 23(10), 792-794 (1998).

[173] K. W. DeLong and R. Trebino, "Improved ultrashort pulse-retrieval algorithm for frequency-resolved optical gating," J. Opt. Soc. Am. A 11(9), 2429-2437 (1994).

[174] K. W. DeLong, R. Trebino, and D. J. Kane, "Comparison of ultrashort-pulse frequency-resolved-optical-gating traces for three common beam geometries," J. Opt. Soc. Am. B 11(9), 1595-1608 (1994).

[175] C. laconis and I. Walmsley, "Self-referencing spectral interferometry for measuring ultrashort optical pulses," IEEE J. Quantum Elect. 35(4), 501509 (1999). 
[176] A. S. Wyatt, I. A. Walmsley, G. Stibenz, and G. Steinmeyer, "Sub-10 fs pulse characterization using spatially encoded arrangement for spectral phase interferometry for direct electric field reconstruction," Opt. Lett. 31(12), 19141916 (2006).

[177] J. R. Birge and F. X. Kärtner, "Analysis and mitigation of systematic errors in spectral shearing interferometry of pulses approaching the single-cycle limit," J. Opt. Soc. Am. B 25(6), A111-A119 (2008).

[178] A. S. Wyatt, "Spectral interferometry for the complete characterisation of near infrared femtosecond and extreme ultraviolet attosecond pulses," Ph.D. thesis, Wolfson College, Oxford (2007).

[179] S. Kane and J. Squier, "Grism-pair stretcher-compressor system for simultaneous second- and third-order dispersion compensation in chirped-pulse amplification," J. Opt. Soc. Am. B 14(3), 661-665 (1997).

[180] N. Forget, V. Crozatier, and T. Oksenhendler, "Pulse-measurement techniques using a single amplitude and phase spectral shaper," J. Opt. Soc. Am. B 27(4), 742-756 (2010).

[181] C. G. D. III, S. Backus, M. M. Murnane, and H. C. Kapteyn, "Ultrabroadband phase-matched optical parametric generation in the ultraviolet by use of guided waves," Opt. Lett. 22(20), 1565-1567 (1997).

[182] J. K. Koga, "Effects of initial focusing on the blueshifting of high-power laser pulses," J. Opt. Soc. Am. B 26(5), 930-938 (2009).

[183] E. A. J. Marcatili and R. A. Schmeltzer, "Hollow Metallic and Dielectric Waveguides for Long Distance Optical Transmission and Lasers," Bell Syst. Tech. J. 43(4), 1783-1809 (1964).

[184] A. Dalgarno and A. E. Kingston, "The Refractive Indices and Verdet Constants of the Inert Gases," Proceedings of the Royal Society of London. Series A. Mathematical and Physical Sciences 259(1298), 424-431 (1960).

[185] D. Li, S. Zeng, Q. Luo, P. Bowlan, V. Chauhan, and R. Trebino, "Propagation dependence of chirp in Gaussian pulses and beams due to angular dispersion," Opt. Lett. 34(7), 962-964 (2009).

[186] S. D. Khan, Y. Cheng, M. Moller, K. Zhao, B. Zhao, M. Chini, G. G. Paulus, and Z. Chang, "Ellipticity dependence of $400 \mathrm{~nm}$-driven high harmonic generation," Appl. Phys. Lett. 99(16), 161106 (2011).

[187] D. J. Kane, A. J. Taylor, R. Trebino, and K. W. DeLong, "Single-shot measurement of the intensity and phase of a femtosecond UV laser pulse with frequency-resolved optical gating," Opt. Lett. 19(14), 1061-1063 (1994).

[188] R. Danielius, A. Piskarskas, P. D. Trapani, A. Andreoni, C. Solcia, and P. Foggi, "Matching of group velocities by spatial walk-off in collinear threewave interaction with tilted pulses," Opt. Lett. 21(13), 973-975 (1996). 
[189] J. H. Marburger, "Self-focusing: Theory." Prog. Quant. Electron. 4, 35-110 (1975).

[190] M. Lenzner, J. Krüger, S. Sartania, Z. Cheng, C. Spielmann, G. Mourou, W. Kautek, and F. Krausz, "Femtosecond Optical Breakdown in Dielectrics," Phys. Rev. Lett. 80(18), 4076-4079 (1998). 


\section{Publications}

- A. Grün, Dane R. Austin, S. L. Cousin, and J. Biegert. Noncollinear nonlinear pulse compression of femtosecond pulses in BBO. In preparation.

- S. M. Teichmann, Dane R. Austin, P. K. Bates, S. L. Cousin, A. Grün, M. Clerici, A. Lotti, D. Faccio, P. Di Trapani, A. Couairon, and J. Biegert. Trajectory interferences in a semi-infinite gas cell. Laser Phys. Lett. 9, 207-211 (2012)

- J. Durá, A. Grün, P. K. Bates, S. M. Teichmann, T. Ergler, A. Senftleben, T. Pflüger, C. D. Schroter, R. Moshammer, J. Ullrich, A. Jaron-Becker, A. Becker, and J. Biegert. Wavelength dependence of the suppressed ionization of molecules in strong laser fields. J. Phys. Chem A 116, 2662-2668 (2012).

- Adam S. Wyatt, A. Grün, P. K. Bates, O. Chalus, J. Biegert, and lan A. Walmsley. Accuracy measurements and improvement for complete characterization of optical pulses from nonlinear processes via multiple spectralshearing interferometry. Opt. Express 19, 25355-25366 (2011).

- S. L. Cousin, N. Forget, A. Grün, P. K. Bates, Dane R. Austin, and J. Biegert. Few-cycle pulse characterization with an acousto-optic pulse shaper. Opt. Lett. 36, 2803-2805 (2011).

- D. Faccio, A. Grün, P. K. Bates, O. Chalus, and J. Biegert. Optical amplification in the near-infrared in gas-filled hollow-core fibers. Opt. Lett. 34(19), 2918-2920 (2009). 